\title{
Chiral Lagrangians with decuplet baryons to one loop
}

\author{
Shao-Zhou Jiang, ${ }^{1,2, *}$ Yan-Rui Liu, ${ }^{3, \dagger}$ Hong-Qian Wang, ${ }^{1}$ and Qin-He Yang ${ }^{1}$ \\ ${ }^{1}$ Department of Physics and GXU-NAOC Center for Astrophysics and Space Sciences, Guangxi University, \\ Nanning, Guangxi 530004, People's Republic of China \\ ${ }^{2}$ Guangxi Key Laboratory for the Relativistic Astrophysics, \\ Nanning, Guangxi 530004, People's Republic of China \\ ${ }^{3}$ School of Physics and Key Laboratory of Particle Physics and Particle Irradiation (MOE), \\ Shandong University, Jinan 250100, People's Republic of China
}

(Received 1 February 2018; published 26 March 2018)

\begin{abstract}
We construct the relativistic chiral Lagrangians with decuplet baryons up to the order $\mathcal{O}\left(p^{4}\right)$ (one loop). For the meson-decuplet-decuplet couplings, there are 1, 13, 55, and 548 terms in the $\mathcal{O}\left(p^{1}\right)-\mathcal{O}\left(p^{4}\right)$ order Lagrangians, respectively. For the meson-octet-decuplet Lagrangians, the number of independent terms from $\mathcal{O}\left(p^{1}\right)$ to $\mathcal{O}\left(p^{4}\right)$ is $1,5,67$, and 611 , respectively. For convenience of application, the $\pi \Delta \Delta$ and $\pi N \Delta$ chiral Lagrangians are picked out. This new form of $\Delta$ Lagrangians is equivalent to the original isovectorisospinor one, and we establish relations between these two forms.
\end{abstract}

DOI: 10.1103/PhysRevD.97.054031

\section{INTRODUCTION}

Chiral perturbation theory (ChPT) is a useful tool to describe low-energy strong interactions of mesons [1-3] and baryons [4]. This effective theory is based on the chiral symmetry of QCD and its spontaneous breaking. The interaction terms and various physical quantities in this theory are organized perturbatively by chiral dimension, the order of $p / \Lambda_{\chi}$ where $p$ represents the typical scale of momentum and $\Lambda_{\chi}$ is the scale of chiral symmetry breaking. Theoretically, the higher the chiral dimension terms, the more precise the obtained results. At present, the chiral Lagrangians containing the pseudoscalar mesons [2,3,5-12] and the ground state baryons [4,13-20] [both $\mathrm{SU}(2)$ and $\mathrm{SU}(3)$ ] have been already constructed to the sixth and fourth order, respectively. Recently, the chiral Lagrangians with $\Delta(1232)$ were also considered up to the fourth chiral order $[21,22]$. For the purpose of application, the current existent chiral Lagrangians are precise enough for theoretical studies on low-energy interactions. However, the above investigations missed a kind of particles, the spin-3/2 hyperons.

In reality, a lot of low-energy QCD problems are related to the chiral Lagrangians with decuplet states which are degenerate with the octet baryons in the large $N_{c}$ limit. Such problems include: the masses of the octet/decuplet

\footnotetext{
*jsz@gxu.edu.cn

yrliu@sdu.edu.cn
}

Published by the American Physical Society under the terms of the Creative Commons Attribution 4.0 International license. Further distribution of this work must maintain attribution to the author(s) and the published article's title, journal citation, and DOI. Funded by SCOAP ${ }^{3}$. baryons and the mass relations between octet/decuplet baryons [23-26], the electromagnetic structures of octet and decuplet baryons (magnetic moments, electric quadrupole moments, and electromagnetic form factors) [27-30], the meson-octet/decuplet scattering processes [31,32], the transitions from decuplet states to octet states [33,34], lattice studies of baryon properties [35-37], and so on. Especially, the studies of the transitions between decuplet and octet baryons can shed light on the possible dibaryons [38]. The lowest-order chiral Lagrangian with decuplet states is obtained easily [39], but we find only fragmentary results for high-order terms in the literature (see the references mentioned above). Such Lagrangians are constructed in order to focus on special problems. A complete and minimal set of Lagrangians with decuplet baryons is still needed. One purpose in this paper is to construct the chiral Lagrangians with the decuplet baryons to one loop (the fourth chiral order) systematically.

In the $\mathrm{SU}(2)$ case, we have obtained the chiral Lagrangians with $\Delta$ up to the order $\mathcal{O}\left(p^{4}\right)$ [22], where we use the isovector-isospinor representation [40] in the isospin space for the Rarita-Schwinger (RS) fields. The application of such Lagrangians is not so convenient in some cases. On the other hand, in the SU(3) case, the decuplet baryons are represented in the flavor space as a totally symmetric tensor $T_{a b c}$. Since the $\Delta$ baryons are members of the decuplet representation, the Lagrangians with $\Delta$ can also be expressed with the symmetric tensor. However, it is apparently not straightforward to make a relation between these two formalisms. Another purpose of the present study is to give new chiral Lagrangians with $\Delta$ in the form of $T_{a b c}(a, b, c=1,2)$ and establish the relations to the former formalism. 
This paper is organized as follows. In Sec. II, we review the building blocks for the construction of the chiral Lagrangians with the mesons, the external sources, and a part of the building blocks with baryon fields. In Sec. III, we present the structures of the chiral Lagrangians and give full building blocks with baryon fields. In Sec. IV, the properties of the building blocks, the linear relations of invariant monomials, and the relations between the original chiral Lagrangians with $\Delta$ and the new forms are given. In Sec. V, we list our results and present some discussions. Section VI is a short summary.

\section{BUILDING BLOCKS IN CONSTRUCTING CHIRAL LAGRANGIANS}

Generally speaking, the constructed Lagrangians in ChPT involve the pseudoscalar mesons, the external sources, the decuplet baryons, and the octet baryons. In this section, we present appropriate building blocks in constructing the chiral Lagrangians. More detailed discussions about them can be found in Refs. [2-6,9,10,12,17,18,20-22]. For the spin-3/2 baryon states, we consider both $S U(3)$ and $S U(2)$ cases. For convenience, we simply call the form of chiral Lagrangians with $\Delta$ in Ref. [22] "original" and those in this paper "new." Needless to say, the new form $S U(2)$ Lagrangians are just selected terms of the $S U(3)$ Lagrangians with decuplet baryons. Hence, in the following parts, we treat them in the same way.

\section{A. Building blocks of the mesons and the external sources}

The QCD Lagrangian $\mathcal{L}$ can be written as

$$
\mathcal{L}=\mathcal{L}_{\mathrm{QCD}}^{0}+\bar{q}\left(\not p+\not d \gamma_{5}-s+i p \gamma_{5}\right) q,
$$

where $\mathcal{L}_{\mathrm{QCD}}^{0}$ is the original QCD Lagrangian and $q$ denotes the quark field. We use $s, p, v^{\mu}$, and $a^{\mu}$ to denote scalar, pseudoscalar, vector, and axial-vector external sources, respectively. Conventionally, the tensor source and the $\theta$ term are ignored. As usual, we consider that only $a^{\mu}$ is traceless in the two-flavor case, but both $a^{\mu}$ and $v^{\mu}$ are traceless in the three-flavor case.

In ChPT, the pseudoscalar mesons (Goldstone bosons) come from the spontaneous breaking of the global symmetry $S U\left(N_{f}\right)_{L} \times S U\left(N_{f}\right)_{R}$ into $S U\left(N_{f}\right)_{V}$. The resulting meson fields are collected in $u$, and it transforms as

$$
u \rightarrow g_{L} u h^{\dagger}=h u g_{R}^{\dagger}
$$

under the chiral rotation, where $g_{L}$ and $g_{R}$ represent elements in $S U\left(N_{f}\right)_{L}$ and $S U\left(N_{f}\right)_{R}$, respectively, and $h$ is a compensator field which is a function of the pion fields.

To construct the chirally invariant Lagrangians involving only meson fields and external sources, the building blocks are usually chosen as

$$
\begin{aligned}
u^{\mu} & =i\left\{u^{\dagger}\left(\partial^{\mu}-i r^{\mu}\right) u-u\left(\partial^{\mu}-i l^{\mu}\right) u^{\dagger}\right\}, \\
\chi_{ \pm} & =u^{\dagger} \chi u^{\dagger} \pm u \chi^{\dagger} u, \\
h^{\mu \nu} & =\nabla^{\mu} u^{\nu}+\nabla^{\nu} u^{\mu}, \\
f_{+}^{\mu \nu} & =u F_{L}^{\mu \nu} u^{\dagger}+u^{\dagger} F_{R}^{\mu \nu} u, \\
f_{-}^{\mu \nu} & =u F_{L}^{\mu \nu} u^{\dagger}-u^{\dagger} F_{R}^{\mu \nu} u=-\nabla^{\mu} u^{\nu}+\nabla^{\nu} u^{\mu},
\end{aligned}
$$

where $r^{\mu}=v^{\mu}+a^{\mu}, l^{\mu}=v^{\mu}-a^{\mu}, \chi=2 B_{0}(s+i p), F_{R}^{\mu \nu}=$ $\partial^{\mu} r^{\nu}-\partial^{\nu} r^{\mu}-i\left[r^{\mu}, r^{\nu}\right], \quad F_{L}^{\mu \nu}=\partial^{\mu} l^{\nu}-\partial^{\nu} l^{\mu}-i\left[l^{\mu}, l^{\nu}\right], \quad$ and $B_{0}$ is a constant related to the quark condensate. The form of these building blocks, however, is not very useful in the construction of chiral Lagrangians with decuplet baryons. For convenience, we write the flavor indices of these building blocks (or any other matrices in the flavor space) explicitly,

$$
X=X_{a}{ }^{b}+X_{s} I, \quad X_{s}=\frac{1}{N_{f}}\langle X\rangle,
$$

where $X$ denotes any building block in Eq. (3) (or any matrix in the flavor space), $X_{a}{ }^{b}\left(X_{s}\right)$ is the traceless (traceable) part of $X, I$ is the $N_{f} \times N_{f}$ identity matrix in the $N_{f}$-flavor space, and $\langle\cdots\rangle$ means the trace in the flavor space. We use $a$ and $b(a, b=1,2,3)$ to denote the row index and column index of the matrix $X$, respectively. In the following, we will treat the row index (or the first index) of $X_{a}{ }^{b}$ as the subscript and the column index (or the second index) as the superscript. According to these notations, we have $u_{s}^{\mu}=f_{-, s}^{\mu \nu}=h_{s}^{\mu \nu}=0$ in the two-flavor case and an additional relation $f_{+, s}^{\mu \nu}=0$ in the three-flavor case. The chiral transformations $(R)$ for these building blocks are

$$
\begin{aligned}
X_{a}{ }^{b} \stackrel{R}{\rightarrow} X_{a}^{\prime b}=h_{a}{ }^{a^{\prime}} X_{a^{\prime}}^{\prime}{ }^{b^{\prime}} h^{\dagger} b^{\prime}, \\
X_{s} \stackrel{R}{\rightarrow} X_{s}^{\prime}=X_{s} .
\end{aligned}
$$

Here $h_{a}{ }^{a^{\prime}}$ does not need to be traceless as the definition of $X_{a}{ }^{b}$ in Eq. (4). The row index of $X_{a}{ }^{b}$ is related to the $h$ field, but the column index is related to the $h^{\dagger}$ field.

The covariant derivative $\nabla^{\mu}$ acting on the building blocks in Eq. (5) is

$$
\begin{aligned}
\nabla^{\mu} X_{a}{ }^{b} & =\partial^{\mu} X_{a}^{b}+\Gamma_{a}^{c, \mu} X_{c}^{b}-X_{a}{ }^{c} \Gamma_{c}^{b, \mu}, \\
\nabla^{\mu} X_{s} & =\partial^{\mu} X_{s}, \\
\Gamma^{\mu} & =\frac{1}{2}\left\{u^{\dagger}\left(\partial^{\mu}-i r^{\mu}\right) u+u\left(\partial^{\mu}-i l^{\mu}\right) u^{\dagger}\right\} .
\end{aligned}
$$

In constructing the Lagrangian, the following two relations will be useful:

$$
\begin{gathered}
{\left[\nabla^{\mu}, \nabla^{\nu}\right] X_{a}^{b}=\Gamma_{a}^{c, \mu \nu} X_{c}^{b}-X_{a}{ }^{c} \Gamma_{c}^{b, \mu \nu},} \\
{\left[\nabla^{\mu}, \nabla^{\nu}\right] X_{s}=0,} \\
\Gamma^{\mu \nu}=\nabla^{\mu} \Gamma^{\nu}-\nabla^{\nu} \Gamma^{\mu}-\left[\Gamma^{\mu}, \Gamma^{\nu}\right]=\frac{1}{4}\left[u^{\mu}, u^{\nu}\right]-\frac{i}{2} f_{+}^{\mu \nu} .
\end{gathered}
$$




\section{B. Building blocks of baryons}

Besides the meson fields and external fields, we also need baryons belonging to $S U(3)$ eight and ten representations. The octet baryons are represented by a matrix $B_{a}{ }^{b}$,

$$
B_{a}{ }^{b}=\left(\begin{array}{ccc}
\frac{\Sigma^{0}}{\sqrt{2}}+\frac{\Lambda}{\sqrt{6}} & \Sigma^{+} & p \\
\Sigma^{-} & -\frac{\Sigma^{0}}{\sqrt{2}}+\frac{\Lambda}{\sqrt{6}} & n \\
\Xi^{-} & \Xi^{0} & -\frac{2 \Lambda}{\sqrt{6}}
\end{array}\right) .
$$

In the two-flavor case, it is reduced to the nucleon doublet,

$$
\psi_{a}=\left(\begin{array}{c}
p \\
n
\end{array}\right) .
$$

One may also use the symbol $B_{a}{ }^{3}(a=1,2)$ to denote this nucleon doublet. For the decuplet baryons, they are denoted by a totally symmetrical tensor $T_{a b c}$ with

$$
\begin{aligned}
& T_{111}=\Delta^{++}, \quad T_{112}=\frac{\Delta^{+}}{\sqrt{3}}, \quad T_{122}=\frac{\Delta^{0}}{\sqrt{3}}, \quad T_{222}=\Delta^{-}, \\
& T_{113}=\frac{\Sigma^{*+}}{\sqrt{3}}, \quad T_{123}=\frac{\Sigma^{* 0}}{\sqrt{6}}, \quad T_{223}=\frac{\Sigma^{*-}}{\sqrt{3}}, \\
& T_{133}=\frac{\Xi^{* 0}}{\sqrt{3}}, \quad T_{233}=\frac{\Xi^{*-}}{\sqrt{3}}, \quad T_{333}=\Omega^{-} .
\end{aligned}
$$

In the $S U(2)$ case, only the first four fields are needed.

The chiral transformations for these baryon fields are

$$
\begin{aligned}
& B_{a}{ }^{b} \stackrel{R}{\rightarrow} B^{\prime}{ }_{a}^{b}=h_{a}{ }^{a^{\prime}} B_{a^{\prime}}{ }^{b^{\prime}} h^{\dagger}{ }_{b^{\prime}}{ }^{b}, \\
& \psi_{a} \stackrel{R}{\longrightarrow} \psi_{a}^{\prime}=h_{a}{ }^{b} \psi_{b}, \\
& T_{a b c} \stackrel{R}{\rightarrow} T_{a b c}^{\prime}=h_{a}{ }^{a^{\prime}} h_{b}{ }^{b^{\prime}} h_{c}{ }^{c^{\prime}} T_{a^{\prime} b^{\prime} c^{\prime}}, \\
& \bar{B}_{a}{ }^{b} \stackrel{R}{\rightarrow} \bar{B}_{a}^{\prime}{ }^{b}=h_{a}{ }^{a^{\prime}} \bar{B}_{a^{\prime}}{ }^{b^{\prime}} h^{\dagger}{ }_{b^{\prime}}{ }^{b}, \\
& \bar{\psi}^{a} \stackrel{R}{\longrightarrow} \psi^{\prime a}=\bar{\psi}^{b} h_{b}^{\dagger}{ }^{a}, \\
& \bar{T}^{a b c} \stackrel{R}{\longrightarrow} \bar{T}^{\prime a b c}=\bar{T}^{a^{\prime} b^{\prime} c^{\prime}} h^{\dagger} a^{\prime}{ }^{a} h^{\dagger} b^{\prime}{ }^{b} h^{\dagger}{ }^{c^{c}} .
\end{aligned}
$$

From the transformations, the indices of $\psi_{a}$ and $T_{a b c}\left(\bar{\psi}^{a}\right.$ and $\bar{T}^{a b c}$ ) can be treated as row (column) indices and those of $B_{a}{ }^{b}$ and $\bar{B}_{a}{ }^{b}$ are self-evident. From Eqs. (5) and (12), we can see that if a term is chirally invariant, all the row indices must be contracted with the column indices and vice versa. This is the reason why we write the row and column indices explicitly.

The covariant derivative $D^{\mu}$ acting on the baryon fields is $[15,18,27]$

$$
\begin{aligned}
D^{\mu} \psi_{a} & =\partial^{\mu} \psi_{a}+\Gamma_{a}^{b, \mu} \psi_{b}, \\
D^{\mu} B_{a}{ }^{b} & =\partial^{\mu} B_{a}{ }^{b}+\Gamma_{a}^{c, \mu} B_{c}{ }^{b}-B_{a}{ }^{c} \Gamma_{c}{ }^{b, \mu}, \\
D^{\mu} T_{a b c} & =\partial^{\mu} T_{a b c}+\Gamma_{a}{ }^{d, \mu} T_{d b c}+\Gamma_{b}{ }^{d, \mu} T_{a d c}+\Gamma_{c}{ }^{d, \mu} T_{a b d} .
\end{aligned}
$$

It seems that, in the three- (two)-flavor case, we can choose $T_{a b c}^{\mu}, \bar{T}^{a b c, \mu}, B_{a}{ }^{b}, \bar{B}_{a}{ }^{b}\left(T_{a b c}^{\mu}, \bar{T}^{a b c, \mu}, \psi_{a}, \bar{\psi}^{a}\right)$, and their covariant derivatives as building blocks, but it is a bit more complex for the spin-3/2 RS fields. We will discuss this issue in the next section.

\section{STRUCTURES OF CHIRAL LAGRANGIANS WITH DECUPLET BARYONS}

A similar discussion in this section has been presented in Ref. [22]. Here we only list the necessary ingredients for the Lagrangian construction. More details can be found in Refs. [17-21,40-56].

In this paper, we adopt the vector-spinor representation $\Psi^{\mu}(\mu=0,1,2,3)$ [41] for the spin-3/2 fields. The general Lagrangian for a free RS field with mass $m$ reads [42]

$$
\begin{aligned}
\mathcal{L}_{\mathrm{f}}= & \bar{\Psi}_{\mu} \Lambda_{A}^{\mu \nu} \Psi_{\nu}, \\
\Lambda_{A}^{\mu \nu}= & -\left[(i \not \partial-m) g^{\mu \nu}+i A\left(\gamma^{\mu} \partial^{\nu}+\gamma^{\nu} \partial^{\mu}\right)\right. \\
& +\frac{i}{2}\left(3 A^{2}+2 A+1\right) \gamma^{\mu} \not \partial \gamma^{\nu} \\
& \left.+m\left(3 A^{2}+3 A+1\right) \gamma^{\mu} \gamma^{\nu}\right],
\end{aligned}
$$

where $A \neq-1 / 2$ is an arbitrary real number. From this Lagrangian, one derives the equation of motion (EOM) and two subsidiary conditions:

$$
\begin{aligned}
(i \not \supset-m) \Psi_{\mu} & =0, \\
\gamma^{\mu} \Psi_{\mu} & =0, \\
\partial^{\mu} \Psi_{\mu} & =0 .
\end{aligned}
$$

The two unphysical spin- $\frac{1}{2}$ degrees of freedom in the vectorspinor representation can be eliminated with these two subsidiary conditions.

There exists a so-called "point" or "contact" transformation under which the above Lagrangian is invariant,

$$
\begin{aligned}
\Psi_{\mu} & \rightarrow \Psi_{\mu}^{\prime}=\Psi_{\mu}+\frac{1}{2} a \gamma_{\mu} \gamma_{\nu} \Psi^{\nu}, \\
A & \rightarrow A^{\prime}=\frac{A-a}{1+2 a}, \quad a \neq-\frac{1}{2} .
\end{aligned}
$$

The choice for the value of $A$ does not affect physical quantities [50,55,57]. Therefore, one may simplify the above Lagrangian by a field redefinition [48],

$$
\begin{aligned}
\mathcal{L}_{\mathrm{f}} & =\bar{\psi}_{A \mu} \Lambda^{\mu \nu} \psi_{A \nu}, \\
\Lambda^{\mu \nu} & =-(i \not \partial-m) g^{\mu \nu}+\frac{1}{4} \gamma^{\mu} \gamma^{\lambda}(i \not \supset-m) \gamma_{\lambda} \gamma^{\nu},
\end{aligned}
$$


where $\psi_{A}^{\mu} \equiv O_{A}^{\mu \nu} \Psi_{\nu}=\left(g^{\mu \nu}+\frac{1}{2} A \gamma^{\mu} \gamma^{\nu}\right) \Psi_{\nu}$. Now, $\Lambda^{\mu \nu}$ is independent of $A$, and the $A$ dependence is implied in $\psi_{A}^{\mu}$.

For the meson-decuplet-decuplet (MTT) interactions, the chiral Lagrangian has the form

$$
\begin{aligned}
\mathcal{L}_{\mathrm{MTT}}= & \bar{T}_{\mu}^{a b c} \Lambda_{A, a b c}^{d e f, \mu \nu} T_{d e f, \nu}, \\
\Lambda_{A, a b c}^{d e f, \mu \nu}= & -\left[\left(i \not D-m_{T}\right) g^{\mu \nu}+i A\left(\gamma^{\mu} D^{\nu}+\gamma^{\nu} D^{\mu}\right)\right. \\
& +\frac{i}{2}\left(3 A^{2}+2 A+1\right) \gamma^{\mu} \not D \gamma^{\nu} \\
& \left.+m_{T}\left(3 A^{2}+3 A+1\right) \gamma^{\mu} \gamma^{\nu}\right] \delta_{a}{ }^{d} \delta_{b}{ }^{e} \delta_{c}{ }^{f} \\
& +O_{1, A, a b c}^{d e f, \mu \nu},
\end{aligned}
$$

where $m_{T}$ is the decuplet mass in the $S U(3)$ limit and $O_{1, A, a b c}^{d e f, \mu \nu}$ contains the meson fields and the external sources. Then the EOM and the subsidiary conditions in ChPT are

$$
\begin{aligned}
&\left(i \not D-m_{T}\right) T_{a b c}^{\mu} \doteq 0, \\
& D_{\mu} T_{a b c}^{\mu} \doteq 0, \\
& \gamma_{\mu} T_{a b c}^{\mu} \doteq 0,
\end{aligned}
$$

where the symbol ".”" means that both sides are equal if high-order terms are ignored. We may write the structure of any term in $O_{1, a b c}^{\text {def, } \mu \nu}$ as $[17,21,22]$

$$
\bar{T}^{a b c, \mu} O \cdots \Theta \cdots T_{d e f}^{\nu}+\text { H.c., }
$$

where $\cdots$ denotes suitable flavor and Lorentz indices, $O \cdots$ is the product of the building blocks with the meson fields and the external sources in Sec. II A, and $\Theta_{\ldots}^{\ldots . .}$ contains a Clifford algebra element $\Gamma \in\left\{1, \gamma_{\mu}, \gamma_{5}, \gamma_{5} \gamma_{\mu}, \sigma_{\mu \nu}\right\}$, the Levi-Civita tensors in Lorentz space $\varepsilon^{\mu \nu \lambda \rho}$, and the covariant derivatives acting on $T_{\text {def }}^{\nu}$. Up to the order $\mathcal{O}\left(p^{4}\right)$, the structures of $\Theta \ldots$ can be found below Eq. (49) in Ref. [22].

With the structure in Eq. (26), the low-energy constants (LECs) in $O_{1, a b c}^{d e f, \mu \nu}$ are dependent on $A$. One can absorb the parameter $A$ into the redefined RS fields according to the point transformation [Eqs. (18) and (20)]. Then the Lagrangian (22) can be rewritten as

$$
\begin{aligned}
\mathcal{L}_{\mathrm{MTT}}= & -\bar{T}_{A, \mu}^{a b c}\left[\left(i \not D-m_{T}\right) g^{\mu \nu}-\frac{1}{4} \gamma^{\mu} \gamma^{\lambda}\left(i \not D-m_{T}\right) \gamma_{\lambda} \gamma^{\nu}\right] \bar{T}_{A, a b c, \nu} \\
& +\bar{T}_{A, \mu}^{a b c} O_{1, a b c}^{\text {def }, \mu \nu} T_{A, \text { def }, \nu},
\end{aligned}
$$

where $T_{A, a b c}^{\mu}=O_{A}^{\mu \nu} T_{a b c, \nu}$. Now, the LECs in Eq. (27) are independent of $A$, but the invariant monomials have the same structures as those in Eq. (22); i.e., one may get Eq. (27) from Eq. (22) by changing $T_{a b c, \mu}$ to $T_{A, a b c, \mu}$ only. The LECs in these two equations are equal if $A=0$.
Physically, we can choose any value of $A(A \neq-1 / 2)$ ( $A=-1$ is a simple and widely used value). In the final results (Sec. V), we only give the structures in Eq. (22).

The new form $\pi \Delta \Delta$ Lagrangians are very similar to the MTT Lagrangians. The differences lie only in the baryon mass and the flavor indices. By changing $m_{T}$ to $m_{\Delta}$ ( $\Delta$ mass in the chiral limit) and limiting all the flavor indices to 1 and 2, the new form of $\pi \Delta \Delta$ Lagrangians is obtained.

For the meson-octet-decuplet and $\pi N \Delta$ interactions, the chiral Lagrangians have the following structures, respectively,

$$
\begin{gathered}
\epsilon^{a b c} \bar{B}_{d}{ }^{e} O \cdots \Theta \cdots T_{A, n, f g h}^{\mu}+\text { H.c., } \\
\epsilon^{a b} \bar{\psi}^{c} O \cdots \Theta \cdots T_{A, n, \text { def }}^{\mu}+\text { H.c. }
\end{gathered}
$$

where $O \ldots$ and $\Theta_{\ldots} \ldots$ have the same meanings as those in Eq. (26). For the Levi-Civita tensor, we have column indices in $\epsilon^{a b c}(a, b, c=1,2,3)$ and row indices in $\epsilon_{a b c}$ (in the H.c. part). Here, $\epsilon^{a b} \equiv \epsilon^{a b 3}$. The RS field depending on $A$ is defined through

$$
\begin{aligned}
T_{A, n, f g h, \mu} & =\Theta_{A, n, \mu \nu}\left(z_{n}\right) T_{f g h}^{\nu}, \\
\Theta_{A, n, \mu \nu}\left(z_{n}\right) & =g_{\mu \nu}+\left[z_{n}+\frac{1}{2}\left(1+4 z_{n}\right) A\right] \gamma_{\mu} \gamma_{\nu} \\
& \equiv \Theta_{n, \mu \alpha}\left(z_{n}\right) O_{A \nu}^{\alpha}=O_{A \mu}{ }^{\alpha} \Theta_{n, \alpha \nu}\left(z_{n}\right), \\
\Theta_{n, \mu \alpha}\left(z_{n}\right) & \equiv g_{\mu \alpha}+z_{n} \gamma_{\mu} \gamma_{\alpha} .
\end{aligned}
$$

Some $z_{n}$ parameters are needed because of the point transformation [58]. They can be obtained from experiments. In Eqs. (28) and (29), the point-invariant structures have been implied and the LECs are already independent of $A$.

To construct Lagrangians, for the baryon fields, we choose $T_{a b c}^{\mu}, \bar{T}^{a b c, \mu}, T_{A, a b c}^{\mu}, \bar{T}_{A}^{a b c, \mu}, B_{a}{ }^{b}, \bar{B}_{a}{ }^{b}$, and their covariant derivatives as building blocks in the three-flavor case. In the two-flavor case, we adopt $T_{a b c}^{\mu}, \bar{T}^{a b c, \mu}, T_{A, a b c}^{\mu}$, $\bar{T}_{A}^{a b c, \mu}, \psi_{a}, \bar{\psi}^{a}$, and their covariant derivatives.

\section{PREPARATIONS FOR LAGRANGIAN CONSTRUCTION}

In this section, we make preparations for the construction of chiral Lagrangians with decuplet baryons. The new form of chiral Lagrangians with $\Delta$ is understood. The recipes are very similar to those in constructing Lagrangians for mesons, meson-baryon systems, and the $\pi-N-\Delta$ systems in Refs. [12,20,22].

\section{A. Power counting and transformation properties}

The chiral dimensions $[2-4,6,17,18]$ of the building blocks with the external sources are listed in the second column of Table I and those of the Clifford algebra and the 
TABLE I. Chiral dimension (Dim), parity $(P)$, charge conjugation $(C)$, and Hermiticity (H.c.) of the building blocks with the external sources.

\begin{tabular}{|c|c|c|c|c|}
\hline & Dim & $P$ & C & H.c. \\
\hline$u_{a}^{b, \mu}$ & 1 & $-u_{a \mu}^{b}$ & $u_{b}^{a, \mu}$ & $u_{a}^{b, \mu}$ \\
\hline$h_{a}^{b, \mu \nu}$ & 2 & $-h_{a}^{b}{ }_{\mu \nu}$ & $h_{b}{ }^{a, \mu \nu}$ & $h_{a}^{b, \mu \nu}$ \\
\hline$\chi_{ \pm, a}^{b}$ & 2 & $\pm \chi_{ \pm, a}{ }^{b}$ & $\chi_{ \pm, b}{ }^{a}$ & $\pm \chi_{ \pm, a}{ }^{b}$ \\
\hline$\chi_{ \pm, s}$ & 2 & $\pm \chi_{ \pm, s}$ & $\chi_{ \pm, s}$ & $\pm \chi_{ \pm, s}$ \\
\hline$f_{ \pm, a} b, \mu \nu$ & 2 & $\pm f_{ \pm, a}{ }^{\prime}{ }_{\mu \nu}$ & $\mp f_{ \pm, b}^{a, \mu \nu}$ & $f_{ \pm, a}, \mu \nu$ \\
\hline$f_{+, s}{ }^{\mu \nu}$ & 2 & $f_{+, s, \mu \nu}$ & $-f_{+, s}{ }^{\mu \nu}$ & $f_{+, s}^{\mu \nu}$ \\
\hline
\end{tabular}

Levi-Civita tensors are given in the second column of Table II $[17,18,56]$. The baryon fields are chiral dimensionless and the information is not shown in these tables. The covariant derivatives acting on the meson fields and the external sources are counted as $\mathcal{O}\left(p^{1}\right)$, but those acting on the baryon fields are counted as $\mathcal{O}\left(p^{0}\right)$.

The chiral Lagrangian should be invariant under the chiral rotation $(R)$, parity transformation $(P)$, charge conjugation transformation $(C)$, and Hermitian transformation (h.c.). The chiral rotations for the building blocks have been discussed in Eqs. (5) and (12). The $P, C$, and h.c. transformations are almost the same as those in Ref. [22] and we also present such properties in Tables I and II. Only different properties will be mentioned.

Compared with Table I of Ref. [22], Table I here shows the flavor indices explicitly. The meanings of plus and minus signs in Table II are the same as those in Refs. [17,20,22]. One thing different is the $\epsilon^{i j k}$. This symbol in Ref. [22] is in the isovector space and it absorbs a minus sign in $C$ transformations (Eq. (31) of Ref. [22]). But now $\epsilon^{a b c}$ and $\epsilon^{a b}$ are the Levi-Civita tensors in the three- (two)-flavor space. They do not need to absorb an extra minus sign.

TABLE II. Chiral dimension (Dim), parity $(P)$, charge conjugation $(C)$, and Hermiticity (H.c.) of the Clifford algebra elements, the Levi-Civita tensors, and the covariant derivatives. The subscript "TT" ("BT") denotes the meson-decuplet-decuplet (meson-octet-decuplet) interactions in the three flavors $(\pi \Delta \Delta$ $(\pi N \Delta)$ interactions in the two-flavor case). $\Psi$ denotes any baryon field, decuplet baryon, $\Delta$, octet baryon, or nucleon. $\epsilon^{a b c}\left(\epsilon^{a b}\right)$ is the Levi-Civita tensor in three- (two)-flavor space. The meaning of the plus or minus sign is explained in the text.

\begin{tabular}{lccccccc}
\hline \hline & Dim & $P_{\mathrm{TT}}$ & $C_{\mathrm{TT}}$ & H.c.. $\mathrm{TT}$ & $P_{\mathrm{BT}}$ & $C_{\mathrm{BT}}$ & H.c. $\cdot \mathrm{BT}$ \\
\hline 1 & 0 & + & + & + & - & + & + \\
$\gamma_{5}$ & 1 & - & + & - & + & + & - \\
$\gamma^{\mu}$ & 0 & + & - & + & - & - & + \\
$\gamma_{5} \gamma^{\mu}$ & 0 & - & + & + & + & + & + \\
$\sigma^{\mu \nu}$ & 0 & + & - & + & - & - & + \\
$\epsilon^{\mu \nu \lambda \rho}$ & 0 & - & + & + & - & + & + \\
$\epsilon^{a b c}$ & 0 & + & + & + & + & + & + \\
$\epsilon^{a b}$ & 0 & + & + & + & + & + & + \\
$D^{\mu} \Psi$ & 0 & + & - & - & + & + & + \\
\hline \hline
\end{tabular}

\section{B. Linear relations}

Some linear relations exist in reducing the chiralinvariant terms to a minimal set. The relations coming from partial integration, EOM, covariant derivatives, and Bianchi identity are the same as those in Ref. [22]. The relations coming from the Cayley-Hamilton relation are the same as those in Ref. [6]. We will not discuss them any more and we only focus on the different and new relations in the following parts.

\section{Schouten identity}

The Schouten identity in the Lorentz space is the same as that in Ref. [22], but some differences exist in the flavor space. For the Levi-Civita tensor $\epsilon^{a b c}\left(\epsilon^{a b}\right)$ in the three- (two)-flavor space, the Schouten identities for any operator $A$ are

$$
\begin{aligned}
& 0=\epsilon_{a b c} A_{d}-\epsilon_{d b c} A_{a}-\epsilon_{a d c} A_{b}-\epsilon_{a b d} A_{c}, \\
& 0=\epsilon_{a b} A_{c}-\epsilon_{c b} A_{a}-\epsilon_{a c} A_{b} .
\end{aligned}
$$

There are two types of indices in $A$ (row or column). Equation (32) works only for the case that the indices in the Levi-Civita tensor and the indices in $A$ are the same type.

\section{Fierz transformations}

The basic Fierz transformation for the Pauli matrices is

$$
\tau_{a b}^{i} \tau_{c d}^{i}=2 \delta_{a d} \delta_{c b}-\delta_{a b} \delta_{c d} .
$$

With this equation, for any two $2 \times 2$ building blocks $X_{a}{ }^{b}$ and $Y_{a}{ }^{b}$ in Table I, one may obtain [59]

$$
\begin{aligned}
X_{a}{ }^{d} Y_{b}{ }^{e}= & \frac{1}{2}\left(Y_{a}{ }^{e} X_{b}{ }^{d}+X_{a}{ }^{e} Y_{b}{ }^{d}+X_{c}{ }^{f} Y_{f}{ }^{c} \delta_{a}{ }^{e} \delta_{b}{ }^{d}\right. \\
& \left.-X_{c}{ }^{f} Y_{f}{ }^{c} \delta_{a}{ }^{d} \delta_{b}{ }^{e}+X_{a}{ }^{c} Y_{c}{ }^{e} \delta_{b}{ }^{d}-\delta_{a}{ }^{e} X_{b}{ }^{c} Y_{c}{ }^{d}\right) .
\end{aligned}
$$

The basic Fierz transformation for the Gall-Mann matrices is

$$
\lambda_{a c}^{i} \lambda_{b d}^{i}=2 \delta_{a d} \delta_{c b}-\frac{2}{3} \delta_{a c} \delta_{b d} .
$$

With the relation in Ref. [60] and the properties of the structure constants of $S U(3)$, one finds that the following relation exists for any two $3 \times 3$ building blocks $X_{a}{ }^{b}$ and $Y_{a}{ }^{b}$ in Table I,

$$
\begin{aligned}
0= & X_{a}{ }^{b} Y_{c}{ }^{d}-X_{a}{ }^{d} Y_{c}{ }^{b}-X_{c}{ }^{b} Y_{a}{ }^{d}+X_{c}{ }^{d} Y_{a}{ }^{b}+X_{a}{ }^{e} Y_{e}{ }^{b} \delta_{c}{ }^{d} \\
& -X_{a}{ }^{e} Y_{e}{ }^{d} \delta_{c}{ }^{b}-X_{c}{ }^{e} Y_{e}{ }^{b} \delta_{a}{ }^{d}+X_{c}{ }^{e} Y_{e}{ }^{d} \delta_{a}{ }^{b}+\delta_{a}{ }^{b} Y_{c}{ }^{e} X_{e}{ }^{d} \\
& -\delta_{a}{ }^{d} Y_{c}{ }^{e} X_{e}{ }^{b}-\delta_{c}{ }^{b} Y_{a}{ }^{e} X_{e}{ }^{d}+\delta_{c}{ }^{d} Y_{a}{ }^{e} X_{e}{ }^{b}-X_{e}{ }^{f} Y_{f}{ }^{e} \delta_{a}{ }^{b} \delta_{c}{ }^{d} \\
& +X_{e}{ }^{f} Y_{f}{ }^{e} \delta_{a}{ }^{d} \delta_{c}{ }^{b} .
\end{aligned}
$$




\section{Contact terms}

The method to construct contact terms is the same as that in Ref. [22]. In the two- (three)-flavor case, the total number of the contact terms is six (five) and we list them in the end of Table V. The last term in Table V is at the $\mathcal{O}\left(p^{6}\right)$ order in the $S U(3)$ case.

\section{Relations between the original chiral Lagrangians with $\Delta$ and the new ones}

In Ref. [22], we have obtained the chiral Lagrangians with $\Delta$ to one loop. There, the $\Delta$ fields are represented by an isovector-isospinor RS field $\psi_{i}^{\mu}(i=1,2,3)$. Now, we use a totally symmetrical tensor $T_{a b c}^{\mu}(a, b, c=1,2)$ to represent them. The difference lies only in the flavor representations. By some calculations, one gets the following relations between these two formalisms of interaction terms,

$$
\begin{aligned}
\bar{T}^{a b c} O T_{a b c}= & \bar{\psi}_{i} O \psi_{i}, \\
\bar{T}^{a b e} O_{e}{ }^{f} T_{a b f}= & \bar{\psi}_{i} O_{j} \tau_{j} \psi_{i}, \\
\bar{T}^{a b c} X_{b}{ }^{e} Y_{c}{ }^{f} T_{a e f}= & \bar{\psi}_{i} X_{j} Y_{j} \psi_{i}-\bar{\psi}_{i} X_{i} Y_{j} \psi_{j} \\
& -\bar{\psi}_{i} X_{j} Y_{i} \psi_{j}, \\
\bar{T}^{a b c} X_{a}{ }^{d} Y_{b}{ }^{e} Z_{c}{ }^{f} T_{d e f}= & \frac{1}{6} \bar{\psi}_{i} X_{l} Y_{j} \tau_{j} Z_{l} \psi_{i} \\
& -\frac{1}{3} \bar{\psi}_{i} X_{i} Y_{k} \tau_{k} Z_{j} \psi_{j} \\
& +P(X, Y, Z), \\
\epsilon^{a b} \bar{\psi}^{c} X_{a}{ }^{e} Y_{c}{ }^{f} T_{e b f}= & \sqrt{2} \bar{\psi} X_{i} Y_{j} \tau_{j} \psi_{i}, \\
\epsilon^{a b} \bar{\psi}^{c} X_{a}{ }^{e} Y_{b}{ }^{f} T_{e f c}= & \sqrt{2} i \epsilon_{i j k} \bar{\psi} X_{i} Y_{j} \psi_{k}, \\
\epsilon^{a b} \bar{\psi}^{c} X_{a}{ }^{d} Y_{b}{ }^{e} Z_{c}{ }^{f} T_{d e f}= & \sqrt{2} i \epsilon_{i j k} \bar{\psi} X_{i} Y_{j} Z_{l} \tau_{l} \psi_{k},
\end{aligned}
$$

where $P(X, Y, Z)$ means all permutations for the symbols $X, Y$, and $Z$. $O, O_{i}, X_{i}, Y_{i}$ and $Z_{i}$ are building blocks in Ref. [22] or their products. The definitions of the symbols in the right-hand side can be found in Ref. [22].

Alternatively, we may transform the original formalism to the new one. To do that, we define transition isospin $I^{j} \quad$ through $\psi_{j}=I_{j} \phi$ with $\phi=\left(\Delta^{++}, \Delta^{+}, \Delta^{0}, \Delta^{-}\right)^{T}$. Similarly, we define $T_{a b c}=W_{a b c}^{i} \phi^{i}$. The matrix forms of $I^{j}$ and the values of $W_{a b c}^{i}$ are easy to obtain from the definitions. We have two relations in connecting the original $\pi \Delta \Delta$ Lagrangians with the new ones,

$$
\left(I_{i}^{\dagger} I_{j}\right)_{x}{ }^{y}=\frac{1}{2}\left[W_{x}^{a b c}\left(\tau_{i} \tau_{j}\right)_{a}{ }^{d} W_{b c d}^{y}-W_{x}^{a b c}\left(\tau_{i}\right)_{a}{ }^{d}\left(\tau_{j}\right)_{b}{ }^{e} W_{c d e}^{y}\right],
$$

$$
\begin{aligned}
\left(I_{i}^{\dagger} \tau_{l} I_{j}\right)_{x}{ }^{y}= & \frac{1}{2}\left[W_{x}^{a b c}\left(\tau_{i} \tau_{j}\right)_{a}{ }^{d}\left(\tau_{l}\right)_{b}{ }^{e} W_{c d e}^{y}\right. \\
& \left.-W_{x}^{a b c}\left(\tau_{i}\right)_{a}{ }^{d}\left(\tau_{j}\right)_{b}{ }^{e}\left(\tau_{l}\right)_{c}{ }^{f} W_{d e f}^{y}\right] .
\end{aligned}
$$

For the special case $j=i$, one has

$$
\begin{aligned}
\left(I_{i}^{\dagger} I_{i}\right)_{x}{ }^{y} & =W_{x}^{a b c} W_{a b c}^{y}, \\
\left(I_{i}^{\dagger} \tau_{l} I_{i}\right)_{x}{ }^{y} & =W_{x}^{a b c}\left(\tau_{l}\right)_{a}{ }^{d} W_{b c d}^{y} .
\end{aligned}
$$

To connect the original $\pi N \Delta$ Lagrangians with the new ones, we may use

$$
\begin{aligned}
\left(I_{i}\right)_{x}{ }^{y} & =\frac{1}{\sqrt{2}} \epsilon^{3 a b}\left(\tau_{i}\right)_{a}{ }^{c} W_{x b c}^{y}, \\
\left(\tau_{i} I_{j}\right)_{x}{ }^{y} & =\frac{1}{\sqrt{2}} \epsilon^{3 a b}\left(\tau_{i}\right)_{x}{ }^{c}\left(\tau_{j}\right)_{a}{ }^{d} W_{b c d}^{y} .
\end{aligned}
$$

Note $\left(\tau_{i} I_{j}\right)_{x}{ }^{y} \neq \frac{1}{\sqrt{2}} \epsilon^{3 a b}\left(\tau_{i}\right)_{a}{ }^{c}\left(\tau_{j}\right)_{x}{ }^{d} W_{b c d}^{y}$. Substituting these six equations into the right-hand sides of Eqs. (37)-(44), one may prove the equivalence of the two sets of relations by using the formula $\epsilon^{3 a b}\left(\tau_{i} \tau_{j}\right)_{a}{ }^{c} W_{x b c}^{y}=\epsilon^{3 a b}\left(\tau_{i}\right)_{a}{ }^{c}\left(\tau_{j}\right)_{b}{ }^{d} W_{x c d}^{y}$.

\section{RESULTS AND DISCUSSIONS}

Following the same steps from Sec. IV C to Sec. IV E in Ref. [22], we obtain the chiral Lagrangians with decuplet baryons up to the order $\mathcal{O}\left(p^{4}\right)$ and list them below.

\section{A. $\mathcal{O}\left(p^{1}\right)$ order}

In the three-flavor case, the lowest order mesondecuplet-decuplet chiral Lagrangian is

$$
\mathcal{L}_{\mathrm{MTT}}^{(1)}=\cdots+C_{1}^{(1)} \bar{T}^{a b c \mu} u_{a}{ }^{d \nu} \gamma_{5} \gamma_{\nu} T_{b c d \mu},
$$

where $C_{1}^{(1)}$ is the low-energy constant at this order and the ellipsis represents the terms coming from the first part in Eq. (22). The lowest order meson-octet-decuplet chiral Lagrangian reads

$$
\mathcal{L}_{\mathrm{MBT}}^{(1)}=D_{1}^{(1)} \epsilon^{a b c} \bar{B}_{a}{ }^{d} u_{b}{ }^{e \mu} T_{c d e \mu}+\text { H.c.. }
$$

In the two-flavor case, the lowest order $\pi \Delta \Delta$ chiral Lagrangian has the same form as Eq. (51),

$$
\mathcal{L}_{\pi \Delta \Delta}^{(1)}=\cdots+e_{1}^{(1)} \bar{T}^{a b c \mu} u_{a}{ }^{d \nu} \gamma_{5} \gamma_{\nu} T_{b c d \mu} .
$$

The difference lies only in the allowed numbers for the indices $a, b, c$, and $d$. Similarly, the lowest order $\pi N \Delta$ chiral Lagrangian can be written as

$$
\mathcal{L}_{\pi N \Delta}^{(1)}=f_{1}^{(1)}\left(\epsilon^{a b} \bar{\psi}^{c} u_{a}^{d \mu} T_{A, n, b c d \mu}+\text { H.c. }\right) .
$$

We have confirmed the previous results in Ref. [22] with the newly constructed Lagrangians. With the relations in 
the last section, we get the relations between these two kinds of LECs,

$$
e_{1}^{(1)}=c_{1}^{(1)}=\frac{1}{2} g_{1}, \quad f_{1}^{(1)}=\frac{1}{\sqrt{2}} g_{\pi N \Delta} .
$$

\section{B. $\mathcal{O}\left(p^{2}\right)$ order}

The $\mathcal{O}\left(p^{2}\right)$ order meson-decuplet-decuplet chiral Lagrangian has the form

$$
\mathcal{L}_{\text {MTT }}^{(2)}=\sum_{n=1}^{13} C_{n}^{(2)} O_{n}^{(3,2)},
$$

where the operators $O_{n}^{\left(N_{f}=3,2\right)}$ are listed in Table III. The meson-octet-decuplet chiral Lagrangian at this order is

$$
\begin{aligned}
\mathcal{L}_{\mathrm{MBT}}^{(2)}= & D_{1}^{(2)}\left(\epsilon^{a b c} \bar{B}_{a}{ }^{d} u_{b}{ }^{e \mu} u_{c}{ }^{f \nu} \gamma_{5} \gamma_{\mu} T_{A, n, d e f \nu}+\text { H.c. }\right) \\
& +D_{2}^{(2)}\left(\epsilon^{a b c} \bar{B}_{a}{ }^{d} u_{b}{ }^{e \mu} u_{d}{ }^{f \nu} \gamma_{5} \gamma_{\mu} T_{A, n, c e f \nu}+\text { H.c. }\right) \\
& +D_{3}^{(2)}\left(\epsilon^{a b c} \bar{B}_{a}{ }^{d} u_{b}{ }^{e \mu} u_{e}{ }^{f \nu} \gamma_{5} \gamma_{\mu} T_{A, n, c d f \nu}+\text { H.c. }\right) \\
& +D_{4}^{(2)}\left(\epsilon^{a b c} \bar{B}_{a}{ }^{d} u_{b}{ }^{e \mu} u_{e}{ }^{f \nu} \gamma_{5} \gamma_{\nu} T_{A, n, c d f \mu}+\text { H.c. }\right) \\
& +D_{5}^{(2)}\left(i \epsilon^{a b c} \bar{B}_{a}{ }^{d} f_{+b}{ }^{e \mu \nu} \gamma_{5} \gamma_{\mu} T_{A, n, c d e \nu}+\text { H.c. }\right) .
\end{aligned}
$$

This result is consistent with that in Ref. [34].

The new form of the $\pi \Delta \Delta$ chiral Lagrangian at the $\mathcal{O}\left(p^{2}\right)$ order is

$$
\mathcal{L}_{\pi \Delta \Delta}^{(2)}=\sum_{n=1}^{11} e_{n}^{(2)} O_{n}^{(2,2)}
$$

TABLE III. The order $\mathcal{O}\left(p^{2}\right)$ meson-decuplet-decuplet $(\pi \Delta \Delta)$ chiral Lagrangians, and the relations between $\pi \Delta \Delta$ LECs here and those in Ref. [22].

\begin{tabular}{lccc}
\hline \hline$O_{n}^{\left(n_{f}, 2\right)}$ & $S U(2)$ & $S U(3)$ & $e_{n}^{(2)}$ \\
\hline $\bar{T}^{a b c \mu} u_{a}{ }^{d}{ }_{\mu} u_{b}{ }^{e \nu} T_{c d e \nu}$ & 1 & 1 & $-c_{1}^{(2)} / 2-c_{2}^{(2)} / 2$ \\
$\bar{T}^{a b c \mu} u_{a}{ }^{d \nu} u_{b}{ }^{e}{ }_{\nu} T_{c d e \mu}$ & 2 & 2 & $-c_{3}^{(2)} / 2$ \\
$\bar{T}^{a b c \mu} u_{a}{ }^{d}{ }_{\mu} u_{d}{ }^{e \nu} T_{b c e \nu}$ & 3 & 3 & $c_{1}^{(2)} / 2+c_{4}^{(2)} / 2$ \\
$\bar{T}^{a b c \mu} u_{a}{ }^{d \nu} u_{d}{ }^{e}{ }_{\mu} T_{b c e \nu}$ & 4 & 4 & $c_{2}^{(2)} / 2+c_{4}^{(2)} / 2$ \\
$\bar{T}^{a b c \mu} u_{a}{ }^{d \nu} u_{d}{ }^{2}{ }_{\nu} T_{b c e \mu}$ & 5 & 5 & $c_{3}^{(2)} / 2+c_{5}^{(2)}$ \\
$\bar{T}^{a b c \mu} u^{d e}{ }_{\mu} u_{e d}{ }^{\nu} T_{a b c \nu}$ & & 6 & \\
$\bar{T}^{a b c \mu} u^{d e \nu} u_{e d \nu} T_{a b c \mu}$ & & 7 & \\
$\bar{T}^{a b c \mu} u_{a}{ }^{d \nu} u_{b}{ }^{e \lambda} D_{\nu \lambda} T_{c d e \mu}$ & 6 & 8 & $-c_{6}^{(2)} / 2$ \\
$\bar{T}^{a b c \mu} u_{a}{ }^{d \nu} u_{d}{ }^{e \lambda} D_{\nu \lambda} T_{b c e \mu}$ & 7 & 9 & $c_{6}^{(2)} / 2+c_{7}^{(2)}$ \\
$\bar{T}^{a b c \mu} u^{d e \nu} u_{e d}{ }^{\lambda} D_{\nu \lambda} T_{a b c \mu}$ & & 10 & \\
$i \bar{T}^{a b c \mu} f_{s,+\mu}{ }^{\nu} T_{a b c \nu}$ & 8 & & $c_{8}^{(2)}$ \\
$i \bar{T}^{a b c \mu} f_{+a}{ }^{\mu}{ }_{\mu}{ }^{\nu} T_{b c d \nu}$ & 9 & 11 & $c_{9}^{(2)}$ \\
$\bar{T}^{a b c \mu} \chi_{+, s} T_{a b c \mu}$ & 10 & 12 & $c_{10}^{(2)}$ \\
$\bar{T}^{a b c \mu} \chi_{+a}{ }^{d} T_{b c d \mu}$ & 11 & 13 & $c_{11}^{(2)}$ \\
\hline \hline
\end{tabular}

where the operators $O_{n}^{\left(N_{f}=2,2\right)}$ can also be found in Table III. The new form $\pi N \Delta$ chiral Lagrangian reads

$$
\begin{aligned}
\mathcal{L}_{\pi N \Delta}^{(2)}= & f_{1}^{(2)}\left(\epsilon^{a b} \bar{\psi}^{c} u_{a}{ }^{d \mu} u_{b}{ }^{e \nu} \gamma_{5} \gamma_{\mu} T_{A, n, c d e \nu}+\text { H.c. }\right) \\
& +f_{2}^{(2)}\left(\epsilon^{a b} \bar{\psi}^{c} u_{a}{ }^{d \mu} u_{c}{ }^{e \nu} \gamma_{5} \gamma_{\mu} T_{A, n, b d e \nu}+\text { H.c. }\right) \\
& +f_{3}^{(2)}\left(i \epsilon^{a b} \bar{\psi}^{c} f_{+a}{ }^{d \mu \nu} \gamma_{5} \gamma_{\mu} T_{A, n, b c d \nu}+\text { H.c. }\right) .
\end{aligned}
$$

This result is consistent with the Lagrangian in Ref. [22]. We present the relations between these two kinds of $\pi \Delta \Delta$ LECs in the last column of Table III. The obtained relations for the $\pi N \Delta$ LECs are

$$
\begin{aligned}
& f_{1}^{(2)}=-\frac{1}{\sqrt{2}} d_{1}^{(2)}, \\
& f_{2}^{(2)}=\frac{1}{\sqrt{2}} d_{1}^{(2)}+\frac{1}{\sqrt{2}} d_{2}^{(2)}, \\
& f_{3}^{(2)}=\frac{1}{\sqrt{2}} d_{3}^{(2)} .
\end{aligned}
$$

\section{C. $\mathcal{O}\left(p^{3}\right)$ and $\mathcal{O}\left(p^{4}\right)$ orders}

We define the $\mathcal{O}\left(p^{3}\right)$ and $\mathcal{O}\left(p^{4}\right)$ chiral Lagrangians as

$$
\begin{aligned}
& \mathcal{L}_{\mathrm{MTT}}^{(m)}=\sum_{n} C_{n}^{(m)} O_{n}^{(3, m)}, \\
& \mathcal{L}_{\mathrm{MBT}}^{(m)}=\sum_{n} D_{n}^{(m)}\left(P_{n}^{(3, m)}+\text { H.c. }\right), \\
& \mathcal{L}_{\pi \Delta \Delta}^{(m)}=\sum_{n} e_{n}^{(m)} O_{n}^{(2, m)}, \\
& \mathcal{L}_{\pi N \Delta}^{(m)}=\sum_{n} f_{n}^{(m)}\left(P_{n}^{(2, m)}+\text { H.c. }\right),
\end{aligned}
$$

where $m=3$ or 4 denotes the chiral dimension, $C_{n}^{(m)}, D_{n}^{(m)}$, $e_{n}^{(m)}$, and $f_{n}^{(m)}$ are the LECs, and $O_{n}^{\left(N_{f}, m\right)}$ and $P_{n}^{\left(N_{f}, m\right)}$ are the independent chiral-invariant terms in the $N_{f}$-flavor case. The results are listed in the Appendix. At the $\mathcal{O}\left(p^{3}\right)$ order, the meson-decuplet-decuplet $(\pi \Delta \Delta)$ Lagrangians are presented in Table IV. There are 55 (38) independent terms in the $S U(3)$ $(S U(2))$ case. The meson-octet-decuplet $(\pi N \Delta)$ Lagrangians are given in Table VI. There are 67 (33) independent terms in the $S U(3)(S U(2))$ case. At the $\mathcal{O}\left(p^{4}\right)$ order, the mesondecuplet-decuplet $(\pi \Delta \Delta)$ Lagrangians are presented in Table V. There are 548 (318) independent terms in the $S U(3)(S U(2))$ case. The meson-octet-decuplet $(\pi N \Delta)$ Lagrangians are listed in Table VII. There are 611 (218) independent terms in the $S U(3)(S U(2))$ case. Note that the $z_{n}$ parameters should be different for the meson-octet-decuplet and $\pi N \Delta$ Lagrangians at the different orders, but we do not distinguish them explicitly in the results.

To merge the meson-octet-decuplet and the $\pi N \Delta$ results, similar to those for the meson-decuplet-decuplet and $\pi \Delta \Delta$, we write them in a unified form. We have changed the 
$S U(2)$ results with $\epsilon^{a b} \bar{\psi}^{c} \rightarrow \epsilon^{d a b} \bar{B}_{d}^{c}$ by setting $d=3$ but $a, b, c=1,2$ as before. Now, one can get the $S U(2)$ results from corresponding terms in Table VI and Table VII with

$$
\epsilon^{a b c} \bar{B}_{a}{ }^{d} \ldots \stackrel{N_{f}=2}{\longrightarrow} \epsilon^{b c} \bar{\psi}^{d} \ldots
$$

Because the number of LECs in $\mathcal{O}\left(p^{3}\right)$ and $\mathcal{O}\left(p^{4}\right)$ Lagrangians is large and only several LECs will be involved in a study, we here do not give the LEC relations between the new and original results at high orders. Each form of Lagrangian can be chosen to study low-energy processes. One may use relations in Sec. IV C to determine LECs from another form terms, if necessary.

From the results, one can see that not only the total number of terms but also the numbers in each type of external source in the chiral Lagrangians with $\Delta$ are the same as those in Ref. [22]. The equality in number is a strict condition for consistency of Lagrangians in different forms. The violation of this condition means that the number of terms in either or both forms is not minimal. This check confirms our previous results.

\section{SUMMARY}

In this paper, we construct the relativistic chiral Lagrangians with decuplet baryons and give a new form of the chiral Lagrangians with $\Delta(1232)$ to one loop. These chiral Lagrangians are for the meson-decupletdecuplet, meson-octet-decuplet, $\pi \Delta \Delta$, and $\pi N \Delta$ interactions. The correspondence between the $\pi \Delta \Delta$ and $\pi N \Delta$ chiral Lagrangians in Ref. [22] and those in the present form can be obtained with the relations we get in Sec. IV C.

\section{ACKNOWLEDGMENTS}

We thank Professor Li-Sheng Geng for useful discussions. Y.-R. L. also thanks the hospitality of Professor M. Oka and other people at Tokyo Institute of Technology where the draft was finished. This work was supported by the National Science Foundation of China (NSFC) under Grants No. 11565004, No. 11775132, No. U1731239, and No. 11673006, the special funding for Guangxi distinguished professors (Bagui Yingcai and Bagui Xuezhe) and the High Level Innovation Team and Outstanding Scholar Program in Guangxi Colleges.

Note added in proof.-After the manuscript was submitted, a work for the next-to-leading order relativistic chiral Lagrangian (Ref. [61]) appeared. The Lagrangians are consistent once the item (vi) below Eq. (46) of Ref. [22] is noted.

\section{APPENDIX: INDEPENDENT TERMS IN $\mathcal{O}\left(p^{3}\right)$ AND $\mathcal{O}\left(p^{4}\right)$ CHIRAL LAGRANGIANS WITH DECUPLET BARYONS}

TABLE IV. Terms in the $\mathcal{O}\left(p^{3}\right)$ meson-decuplet-decuplet and $\pi \Delta \Delta$ chiral Lagrangians, where $O_{n}^{\left(N_{f}, 3\right)}$ is defined in Eqs. (63) and (65).

\begin{tabular}{|c|c|c|c|c|c|}
\hline$O_{n}^{\left(N_{f}, 3\right)}$ & $S U(2)$ & $S U(3)$ & $O_{n}^{\left(N_{f}, 3\right)}$ & $S U(2)$ & $S U(3)$ \\
\hline$\overline{\bar{T}^{a b c \mu} u_{a}{ }_{\mu}^{d} u_{b}{ }^{e \nu} u_{c}{ }^{f \lambda} \gamma_{5} \gamma_{\nu} T_{\text {def } \lambda}}$ & 1 & 1 & $\bar{T}^{a b c \mu} u_{a}{ }^{d \nu} f_{-d{ }^{e} \mu}{ }^{\lambda} D_{\nu} T_{b c e \lambda}+$ H.c. & 15 & 30 \\
\hline $\bar{T}^{a b c \mu} u_{a}{ }^{d}{ }_{\mu} u_{b}{ }^{e \nu} u_{d}{ }^{f \lambda} \gamma_{5} \gamma_{\nu} T_{c e f \lambda}$ & 2 & 2 & $\bar{T}^{a b c \mu} u_{a}{ }^{d \nu} f_{-d^{e}{ }_{\mu}{ }^{\lambda} D_{\lambda} T_{b c e \nu}}+$ H.c. & 16 & 31 \\
\hline $\bar{T}^{a b c \mu} u_{a}{ }^{d}{ }_{\mu} u_{b}{ }^{e \nu} u_{d}{ }^{f \lambda} \gamma_{5} \gamma_{\lambda} T_{c e f \nu}+$ H.c. & 3 & 3 & $\bar{T}^{a b c \mu} u_{a}{ }^{d \nu} f_{-d}{ }^{\mu}{ }_{\nu}{ }^{\lambda} D_{\lambda} T_{b c e \mu}+$ H.c. & 17 & 32 \\
\hline $\bar{T}^{a b c \mu} u_{a}{ }^{d}{ }_{\mu} u_{b}{ }^{e \nu} u_{e}{ }^{f \lambda} \gamma_{5} \gamma_{\lambda} T_{c d f \nu}+$ H.c. & 4 & 4 & $\bar{T}^{a b c \mu} u^{d e}{ }_{\mu} f_{-e d}{ }^{\nu \lambda} D_{\nu} T_{a b c \lambda}+$ H.c. & & 33 \\
\hline $\bar{T}^{a b c \mu} u_{a}{ }^{d \nu} u_{b}{ }^{e}{ }_{\nu} u_{c}{ }^{f \lambda} \gamma_{5} \gamma_{\lambda} T_{\text {def } \mu}$ & 5 & 5 & $\bar{T}^{a b c \mu} u^{d e \nu} f_{-e d \mu}{ }^{\lambda} D_{\nu} T_{a b c \lambda}$ & & 34 \\
\hline $\bar{T}^{a b c \mu} u_{a}{ }^{d}{ }_{\mu} u_{d}{ }^{e \nu} u_{e}{ }^{f \lambda} \gamma_{5} \gamma_{\nu} T_{b c f \lambda}$ & & 6 & $\bar{T}^{a b c \mu} u_{a}{ }^{d}{ }_{\mu} h_{d}{ }^{e \nu \lambda} D_{\nu} T_{b c e \lambda}+$ H.c. & 18 & 35 \\
\hline $\bar{T}^{a b c \mu} u_{a}{ }^{d \nu} u_{b}{ }^{e}{ }_{\nu} u_{d}{ }^{f \lambda} \gamma_{5} \gamma_{\lambda} T_{c e f \mu}+$ H.c. & 6 & 7 & $\bar{T}^{a b c \mu} u_{a}^{d \nu} h_{d}{ }^{e}{ }_{\nu}^{\lambda} D_{\lambda} T_{b c e \mu}+$ H.c. & 19 & 36 \\
\hline $\bar{T}^{a b c \mu} u_{a}{ }^{d \nu} u_{b}{ }^{e \lambda} u_{d}{ }^{f}{ }_{\mu} \gamma_{5} \gamma_{\lambda} T_{c e f \nu}$ & 7 & 8 & $\bar{T}^{a b c \mu} u_{a}{ }^{d \nu} h_{d}^{e \lambda \rho} D_{\nu \lambda \rho} T_{b c e \mu}+$ H.c. & 20 & 37 \\
\hline $\bar{T}^{a b c \mu} u_{a}{ }^{d}{ }_{\mu} u_{d}{ }^{e \nu} u_{e}{ }^{f \lambda} \gamma_{5} \gamma_{\lambda} T_{b c f \nu}+$ H.c. & & 9 & $\bar{T}^{a b c \mu} \nabla^{\nu} f_{-a \nu}^{d}{ }^{\lambda} \gamma_{5} \gamma_{\lambda} T_{b c d \mu}$ & 21 & 38 \\
\hline $\bar{T}^{a b c \mu} u_{a}{ }^{d \nu} u_{b}{ }^{e \lambda} u_{d}{ }^{f}{ }_{\nu} \gamma_{5} \gamma_{\lambda} T_{c e f \mu}$ & 8 & 10 & $i \bar{T}^{a b c \mu} f_{+a}{ }^{d}{ }_{\mu}{ }^{\nu} u_{b}{ }^{e \lambda} \gamma_{5} \gamma_{\nu} T_{c d e \lambda}+$ H.c. & 22 & 39 \\
\hline $\bar{T}^{a b c \mu} u_{a}{ }^{d}{ }_{\mu} u^{e f \nu} u_{f e}{ }^{\lambda} \gamma_{5} \gamma_{\nu} T_{b c d \lambda}+$ H.c. & & 11 & $i \bar{T}^{a b c \mu} f_{+a}{ }^{\mu}{ }_{\mu} \nu u_{b}{ }^{e \lambda} \gamma_{5} \gamma_{\lambda} T_{c d e \nu}$ & 23 & 40 \\
\hline $\bar{T}^{a b c \mu} u_{a}{ }^{d \nu} u_{d}{ }^{e}{ }_{\mu} u_{e}{ }^{f \lambda} \gamma_{5} \gamma_{\lambda} T_{b c f \nu}+$ H.c. & & 12 & $i \bar{T}^{a b c \mu} f_{+a}{ }^{\mu}{ }_{\mu}^{\nu} u_{d}{ }^{e \lambda} \gamma_{5} \gamma_{\nu} T_{b c e \lambda}+$ H.c. & 24 & 41 \\
\hline $\bar{T}^{a b c \mu} u_{a}{ }^{d \nu} u_{d}{ }^{e}{ }_{\nu} u_{e} e^{f \lambda} \gamma_{5} \gamma_{\lambda} T_{b c f \mu}+$ H.c. & & 13 & $i \bar{T}^{a b c \mu} f_{+a}{ }^{\mu}{ }_{\mu}{ }^{\nu} u_{d}{ }^{e \lambda} \gamma_{5} \gamma_{\lambda} T_{b c e \nu}+$ H.c. & 25 & 42 \\
\hline $\bar{T}^{a b c \mu} u_{a}{ }^{d \nu} u_{d}{ }^{e \lambda} u_{e}^{f}{ }_{\mu} \gamma_{5} \gamma_{\lambda} T_{b c f \nu}$ & & 14 & $i \bar{T}^{a b c \mu} f_{+a}{ }^{d \nu \lambda} u_{d}{ }^{e}{ }_{\mu} \gamma_{5} \gamma_{\nu} T_{b c e \lambda}+$ H.c. & 26 & 43 \\
\hline $\bar{T}^{a b c \mu} u_{a}{ }^{d \nu} u_{d}{ }^{e \lambda} u_{e}{ }^{f}{ }_{\nu} \gamma_{5} \gamma_{\lambda} T_{b c f \mu}$ & & 15 & $i \bar{T}^{a b c \mu} f_{+a}{ }^{d \nu \lambda} u_{d}{ }^{e}{ }_{\nu} \gamma_{5} \gamma_{\lambda} T_{b c e \mu}+$ H.c. & 27 & 44 \\
\hline $\bar{T}^{a b c \mu} u_{a}{ }^{d \nu} u^{e f}{ }_{\mu} u_{f e}{ }^{\lambda} \gamma_{5} \gamma_{\nu} T_{b c d \lambda}$ & & 16 & $i \bar{T}^{a b c \mu} f_{+}{ }^{d e}{ }_{\mu}{ }^{\nu} u_{e d}{ }^{\lambda} \gamma_{5} \gamma_{\nu} T_{a b c \lambda}+$ H.c. & & 45 \\
\hline $\bar{T}^{a b c \mu} u_{a}{ }^{d \nu} u^{e f}{ }_{\nu} u_{f e}{ }^{\lambda} \gamma_{5} \gamma_{\lambda} T_{b c d \mu}$ & & 17 & $i \bar{T}^{a b c \mu} f_{+}{ }^{d e}{ }_{\mu}^{\nu}{ }^{\nu} u_{e d}{ }^{\lambda} \gamma_{5} \gamma_{\lambda} T_{a b c \nu}$ & & 46 \\
\hline
\end{tabular}


TABLE IV. (Continued)

\begin{tabular}{|c|c|c|c|c|c|}
\hline$O_{n}^{\left(N_{f}, 3\right)}$ & $S U(2)$ & $S U(3)$ & $O_{n}^{\left(N_{f}, 3\right)}$ & $S U(2)$ & $S U(3)$ \\
\hline $\bar{T}^{a b c \mu} u_{a}{ }^{d \nu} u^{e f \lambda} u_{f e \lambda} \gamma_{5} \gamma_{\nu} T_{b c d \mu}$ & & 18 & $i \bar{T}^{a b c \mu} f_{+a}{ }^{d \nu \lambda} u_{d}{ }^{e \rho} \gamma_{5} \gamma_{\nu} D_{\lambda \rho} T_{b c e \mu}+$ H.c. & 28 & 47 \\
\hline $\bar{T}^{a b c \mu} u^{d e}{ }_{\mu} u_{e}{ }^{f \nu} u_{f d}{ }^{\lambda} \gamma_{5} \gamma_{\nu} T_{a b c \lambda}$ & & 19 & $i \bar{T}^{a b c \mu} f_{s,+\mu}{ }^{\nu} u_{a}{ }^{d \lambda} \gamma_{5} \gamma_{\nu} T_{b c d \lambda}+$ H.c. & 29 & \\
\hline $\bar{T}^{a b c \mu} u_{a}{ }^{d \nu} u_{b}{ }^{e \lambda} u_{c}{ }^{f \rho} \gamma_{5} \gamma_{\nu} D_{\lambda \rho} T_{\text {def } \mu}$ & 9 & 20 & $i \bar{T}^{a b c \mu} f_{s,+\mu}{ }^{\nu} u_{a} d \lambda \gamma_{5} \gamma_{\lambda} T_{b c d \nu}$ & 30 & \\
\hline $\bar{T}^{a b c \mu} u_{a}{ }^{d \nu} u_{b}{ }^{e \lambda} u_{d}^{f \rho} \gamma_{5} \gamma_{\nu} D_{\lambda \rho} T_{c e f \mu}+$ H.c. & 10 & 21 & $i \bar{T}^{a b c \mu} \nabla^{\nu} f_{+a}^{d}{ }_{\nu}{ }^{\lambda} D_{\lambda} T_{b c d \mu}$ & 31 & 48 \\
\hline $\bar{T}^{a b c \mu} u_{a}{ }^{d \nu} u_{b}{ }^{e \lambda} u_{d}^{f \rho} \gamma_{5} \gamma_{\lambda} D_{\nu \rho} T_{c e f \mu}$ & 11 & 22 & $i \bar{T}^{a b c \mu} \nabla^{\nu} f_{s,+\nu}{ }^{\lambda} D_{\lambda} T_{a b c \mu}$ & 32 & \\
\hline $\bar{T}^{a b c \mu} u_{a}{ }^{d \nu} u_{d}{ }^{e \lambda} u_{e}^{f \rho} \gamma_{5} \gamma_{\nu} D_{\lambda \rho} T_{b c f \mu}+$ H.c. & & 23 & $\bar{T}^{a b c \mu} u_{a}^{d \nu} \chi_{+b}^{e} \gamma_{5} \gamma_{\nu} T_{c d e \mu}$ & 33 & 49 \\
\hline $\bar{T}^{a b c \mu} u_{a}{ }^{d \nu} u_{d}{ }^{e \lambda} u_{e}{ }^{f \rho} \gamma_{5} \gamma_{\lambda} D_{\nu \rho} T_{b c f \mu}$ & & 24 & $\bar{T}^{a b c \mu} u_{a}{ }^{d \nu} \chi_{+d}{ }^{e} \gamma_{5} \gamma_{\nu} T_{b c e \mu}+$ H.c. & 34 & 50 \\
\hline $\bar{T}^{a b c \mu} u_{a}{ }^{d \nu} u^{e f \lambda} u_{f e}{ }^{\rho} \gamma_{5} \gamma_{\nu} D_{\lambda \rho} T_{b c d \mu}$ & & 25 & $\bar{T}^{a b c \mu} u^{d e \nu} \chi_{+e d} \gamma_{5} \gamma_{\nu} T_{a b c \mu}$ & & 51 \\
\hline $\bar{T}^{a b c \mu} u_{a}{ }^{d \nu} u^{e f \lambda} u_{f e}{ }^{\rho} \gamma_{5} \gamma_{\lambda} D_{\nu \rho} T_{b c d \mu}$ & & 26 & $\bar{T}^{a b c \mu} u_{a}^{d \nu} \chi_{+, s} \gamma_{5} \gamma_{\nu} T_{b c d \mu}$ & 35 & 52 \\
\hline $\bar{T}^{a b c \mu} u_{a}{ }^{d} f_{-b}{ }^{e \nu \lambda} D_{\nu} T_{c d e \lambda}+$ H.c. & 12 & 27 & $i \bar{T}^{a b c \mu} u_{a}^{d \nu} \chi_{-d}^{e} D_{\nu} T_{b c e \mu}+$ H.c. & 36 & 53 \\
\hline $\bar{T}^{a b c \mu} u_{a}{ }^{d \nu} f_{-b}{ }^{e}{ }_{\mu}^{\lambda} D_{\nu} T_{c d e \lambda}$ & 13 & 28 & $i \bar{T}^{a b c \mu} \nabla^{\nu} \chi_{-a}^{d} \gamma_{5} \gamma_{\nu} T_{b c d \mu}$ & 37 & 54 \\
\hline $\bar{T}^{a b c \mu} u_{a}{ }^{d} f_{-d}{ }^{e \nu \lambda} D_{\nu} T_{b c e \lambda}+$ H.c. & 14 & 29 & $i \bar{T}^{a b c \mu} \nabla^{\nu} \chi_{-, s} \gamma_{5} \gamma_{\nu} T_{a b c \mu}$ & 38 & 55 \\
\hline
\end{tabular}

TABLE V. Terms in the $\mathcal{O}\left(p^{4}\right)$ meson-decuplet-decuplet and $\pi \Delta \Delta$ chiral Lagrangians, where $O_{n}^{\left(N_{f}, 4\right)}$ is defined in Eqs. (63) and (65).

\begin{tabular}{|c|c|c|c|c|c|}
\hline$\overline{O_{n}^{\left(N_{f}, 4\right)}}$ & $S U(2)$ & $S U(3)$ & $O_{n}^{\left(N_{f}, 4\right)}$ & $S U(2)$ & $S U(3)$ \\
\hline $\bar{T}^{a b c \mu} u_{a}{ }^{d}{ }_{\mu} u_{b}{ }^{e \nu} u_{c}{ }^{f}{ }_{\nu} u_{d}{ }^{g \lambda} T_{e f g \lambda}$ & 1 & 1 & $\bar{T}^{a b c \mu} h^{d e \nu \lambda} h_{e d}^{\rho \sigma} D_{\nu \lambda \rho \sigma} T_{a b c \mu}$ & & 299 \\
\hline $\bar{T}^{a b c \mu} u_{a}{ }^{d}{ }_{\mu} u_{b}{ }^{e \nu} u_{c}{ }^{f}{ }_{\nu} u_{e}{ }^{g \lambda} T_{d f g \lambda}+$ H.c. & 2 & 2 & $i \bar{T}^{a b c \mu} h_{a}{ }^{d}{ }_{\mu}^{\nu} h_{d}{ }^{e \lambda \rho} \sigma_{\nu \lambda} T_{b c e \rho}$ & 122 & 300 \\
\hline $\bar{T}^{a b c \mu} u_{a}{ }^{d}{ }_{\mu} u_{b}{ }^{e \nu} u_{c}{ }^{f \lambda} u_{e}{ }^{g}{ }_{\nu} T_{d f g \lambda}$ & 3 & 3 & $\bar{T}^{a b c \mu} u_{a}{ }^{d}{ }_{\mu} \nabla^{\nu} f_{-b}{ }^{e}{ }_{\nu}^{\lambda} T_{c d e \lambda}+$ H.c. & 123 & 301 \\
\hline $\bar{T}^{a b c \mu} u_{a}{ }^{d}{ }_{\mu} u_{b}{ }^{e \nu} u_{c}{ }^{f \lambda} u_{e}{ }_{\lambda}{ }_{\lambda} T_{d f g \nu}+$ H.c. & 4 & 4 & $\bar{T}^{a b c \mu} u_{a}{ }^{d \nu} \nabla^{\lambda} f_{-b}{ }^{e}{ }_{\nu \lambda} T_{c d e \mu}$ & 124 & 302 \\
\hline $\bar{T}^{a b c \mu} u_{a}{ }^{d}{ }_{\mu} u_{b}{ }^{e \nu} u_{d}{ }^{f}{ }_{\nu} u_{e}{ }^{g \lambda} T_{c f g \lambda}$ & 5 & 5 & $\bar{T}^{a b c \mu} u_{a}{ }^{d}{ }_{\mu} \nabla^{\nu} f_{-d}{ }^{e}{ }^{\lambda} T_{b c e \lambda}+$ H.c. & 125 & 303 \\
\hline $\bar{T}^{a b c \mu} u_{a}{ }^{d}{ }_{\mu} u_{b}{ }^{e \nu} u_{d}{ }^{f \lambda} u_{e}{ }_{\nu}{ }_{\nu} T_{c f g \lambda}$ & & 6 & $\bar{T}^{a b c \mu} u_{a}{ }^{d \nu} \nabla^{\lambda} f_{-d}{ }^{e} \mu \lambda T_{b c e \nu}+$ H.c. & 126 & 304 \\
\hline $\bar{T}^{a b c \mu} u_{a}{ }^{d}{ }_{\mu} u_{b}{ }^{e \nu} u_{d}{ }_{\nu}^{f} u_{f}{ }^{g \lambda} T_{c e g \lambda}$ & & 7 & $\bar{T}^{a b c \mu} u_{a}{ }^{d \nu} \nabla^{\lambda} f_{-d}{ }^{e}{ }_{\nu \lambda} T_{b c e \mu}+$ H.c. & 127 & 305 \\
\hline $\bar{T}^{a b c \mu} u_{a}{ }^{d}{ }_{\mu} u_{b}{ }^{e \nu} u_{d}{ }^{f \lambda} u_{e}{ }_{\lambda}{ }_{\lambda} T_{c f g \nu}+$ H.c. & 6 & 8 & $\bar{T}^{a b c \mu} u^{d e}{ }_{\mu} \nabla^{\nu} f_{-e d \nu}^{\lambda} T_{a b c \lambda}+$ H.c. & & 306 \\
\hline $\bar{T}^{a b c \mu} u_{a}{ }^{d}{ }_{\mu} u_{b}{ }^{e \nu} u_{d}{ }^{f \lambda} u_{f}{ }^{g}{ }_{\nu} T_{c e g \lambda}+$ H.c. & & 9 & $\bar{T}^{a b c \mu} u^{d e \nu} \nabla^{\lambda} f_{-e d \nu \lambda} T_{a b c \mu}$ & & 307 \\
\hline $\bar{T}^{a b c \mu} u_{a}{ }^{d}{ }_{\mu} u_{b}{ }^{e \nu} u_{e}{ }^{f}{ }_{\nu} u_{f}{ }^{g \lambda} T_{c d g \lambda}+$ H.c. & & 10 & $\bar{T}^{a b c \mu} u_{a}{ }^{d \nu} \nabla^{\lambda} f_{-b}{ }^{e}{ }^{\rho}{ }^{\rho} D_{\nu \rho} T_{c d e \mu}$ & 128 & 308 \\
\hline $\bar{T}^{a b c \mu} u_{a}{ }^{d}{ }_{\mu} u_{b}{ }^{e \nu} u_{e}{ }^{f \lambda} u_{f}{ }_{\nu}{ }_{\nu} T_{c d g \lambda}+$ H.c. & & 11 & $\bar{T}^{a b c \mu} u_{a}{ }^{d \nu} \nabla^{\lambda} f_{-d}{ }^{e}{ }^{\rho}{ }^{\rho} D_{\nu \rho} T_{b c e \mu}+$ H.c. & 129 & 309 \\
\hline $\bar{T}^{a b c \mu} u_{a}{ }^{d}{ }_{\mu} u_{b}{ }^{e \nu} u_{e}{ }^{f \lambda} u_{f}{ }_{\lambda}^{g} T_{c d g \nu}+$ H.c. & & 12 & $\bar{T}^{a b c \mu} u^{d e \nu} \nabla^{\lambda} f_{-e d \lambda}{ }^{\rho} D_{\nu \rho} T_{a b c \mu}$ & & 310 \\
\hline $\bar{T}^{a b c \mu} u_{a}{ }^{d}{ }_{\mu} u_{b}{ }^{e \nu} u^{f g}{ }_{\nu} u_{g f}{ }^{\lambda} T_{c d e \lambda}+$ H.c. & & 13 & $i \bar{T}^{a b c \mu} f_{+a_{\mu}{ }^{\nu}{ }^{\nu} u_{b}{ }^{e}{ }_{\nu} u_{c}{ }^{f \lambda} T_{\operatorname{def} \lambda}+\text { H.c. }}$ & 130 & 311 \\
\hline $\bar{T}^{a b c \mu} u_{a}{ }^{d \nu} u_{b}{ }_{\nu}^{e} u_{c}{ }^{f \lambda} u_{f}{ }_{\mu}^{g} T_{\operatorname{deg} \lambda}$ & 7 & 14 & $i \bar{T}^{a b c \mu} f_{+a}{ }^{d}{ }_{\mu}^{\nu} u_{b}{ }^{e}{ }_{\nu} u_{d}{ }^{f \lambda} T_{c e f \lambda}+$ H.c. & 131 & 312 \\
\hline $\bar{T}^{a b c \mu} u_{a \mu}{ }^{d} u_{b}{ }^{e \nu} u^{f g \lambda} u_{g f \lambda} T_{c d e \nu}$ & & 15 & $i \bar{T}^{a b c \mu} f_{+a}{ }^{d}{ }_{\mu}^{\nu} u_{b}{ }^{e}{ }_{\nu} u_{e}{ }^{f \lambda} T_{c d f \lambda}+$ H.c. & 132 & 313 \\
\hline $\bar{T}^{a b c \mu} u_{a}{ }^{d \nu} u_{b}{ }^{e}{ }_{\nu} u_{c}{ }^{f \lambda} u_{d}{ }^{g} T_{e f g \mu}$ & 8 & 16 & $i \bar{T}^{a b c \mu} f_{+a}{ }^{d \mu}{ }_{\mu}^{\nu} u_{b}{ }^{e \lambda} u_{c}{ }^{f}{ }_{\lambda} T_{d e f \nu}$ & 133 & 314 \\
\hline $\bar{T}^{a b c \mu} u_{a}{ }^{d \nu} u_{b}{ }^{e}{ }_{\nu} u_{c}{ }^{f \lambda} u_{f}{ }_{\lambda} T_{\text {deg } \mu}$ & 9 & 17 & $i \bar{T}^{a b c \mu} f_{+a}{ }^{d}{ }_{\mu}{ }^{\nu} u_{b}{ }^{e \lambda} u_{d}{ }_{\nu}^{f} T_{c e f \lambda}+$ H.c. & 134 & 315 \\
\hline $\bar{T}^{a b c \mu} u_{a}{ }^{d \nu} u_{b}{ }^{e}{ }_{\nu} u_{d}{ }^{f \lambda} u_{f}{ }_{\mu}{ }_{\mu} T_{c e g \lambda}+$ H.c. & & 18 & $i \bar{T}^{a b c \mu} f_{+a}{ }^{d}{ }_{\mu}^{\nu} u_{b}{ }^{e \lambda} u_{d}{ }_{\lambda}^{f} T_{c e f \nu}+$ H.c. & 135 & 316 \\
\hline $\bar{T}^{a b c \mu} u_{a}{ }^{d}{ }_{\mu} u_{d}{ }^{e \nu} u_{e}{ }_{\nu}^{f} u_{f}{ }^{g \lambda} T_{b c g \lambda}$ & & 19 & $i \bar{T}^{a b c \mu} f_{+a}{ }^{d}{ }_{\mu}^{\nu} u_{b}{ }^{e \lambda} u_{e}{ }_{\nu}{ }_{\nu} T_{c d f \lambda}+$ H.c. & 136 & 317 \\
\hline $\bar{T}^{a b c \mu} u_{a}{ }^{d \nu} u_{b}{ }^{e}{ }_{\nu} u_{d}{ }^{f \lambda} u_{e}{ }^{g}{ }_{\lambda} T_{c f g \mu}$ & 10 & 20 & $i \bar{T}^{a b c \mu} f_{+a}{ }^{d}{ }_{\mu}^{\nu} u_{b}{ }^{e \lambda} u_{e}{ }_{\lambda} T_{c d f \nu}$ & 137 & 318 \\
\hline $\bar{T}^{a b c \mu} u_{a}{ }^{d \nu} u_{b}{ }^{e \lambda} u_{d}{ }^{f}{ }_{\mu} u_{e}{ }^{g}{ }_{\nu} T_{c f g \lambda}$ & 11 & 21 & $i \bar{T}^{a b c \mu} f_{+a}{ }^{d}{ }_{\mu}^{\nu} u_{d}{ }^{e}{ }_{\nu} u_{e}{ }^{f \lambda} T_{b c f \lambda}+$ H.c. & & 319 \\
\hline $\bar{T}^{a b c \mu} u_{a}{ }^{d \nu} u_{b}{ }^{e}{ }_{\nu} u_{d}{ }^{f \lambda} u_{f}{ }_{\lambda}{ }_{\lambda} T_{c e g \mu}+$ H.c. & & 22 & $i \bar{T}^{a b c \mu} f_{+a}{ }^{d \nu \lambda} u_{b}{ }^{e}{ }_{\mu} u_{d}{ }^{f}{ }_{\nu} T_{c e f \lambda}+$ H.c. & 138 & 320 \\
\hline $\bar{T}^{a b c \mu} u_{a}{ }^{d}{ }_{\mu} u_{d}{ }^{e \nu} u_{e}{ }^{f \lambda} u_{f}{ }_{\nu}^{g} T_{b c g \lambda}+$ H.c. & & 23 & $i \bar{T}^{a b c \mu} f_{+a}{ }^{d}{ }_{\mu}^{\nu} u_{d}{ }^{e \lambda} u_{e}{ }^{f}{ }_{\nu} T_{b c f \lambda}+$ H.c. & & 321 \\
\hline $\bar{T}^{a b c \mu} u_{a}{ }^{d \nu} u_{b}{ }^{e \lambda} u_{d}{ }^{f}{ }_{\mu} u_{e}{ }_{\lambda} T_{c f g \nu}$ & & 24 & $i \bar{T}^{a b c \mu} f_{+a}{ }^{d}{ }_{\mu}^{\nu} u_{d}{ }^{e \lambda} u_{e}{ }_{\lambda}{ }_{\lambda} T_{b c f \nu}+$ H.c. & & 322 \\
\hline $\bar{T}^{a b c \mu} u_{a}{ }^{d}{ }_{\mu} u_{d}{ }^{e \nu} u_{e}{ }^{f \lambda} u_{f}{ }_{\lambda}^{g} T_{b c g \nu}+$ H.c. & & 25 & $i \bar{T}^{a b c \mu} f_{+a}{ }^{d \nu \lambda} u_{b}{ }^{e}{ }_{\nu} u_{d}{ }^{f}{ }_{\mu} T_{c e f \lambda}+$ H.c. & 139 & 323 \\
\hline $\bar{T}^{a b c \mu} u_{a}{ }^{d \nu} u_{b}{ }_{\nu}{ }_{\nu} u^{f g}{ }_{\mu} u_{g f}{ }^{\lambda} T_{c d e \lambda}$ & & 26 & $i \bar{T}^{a b c \mu} f_{+a}{ }^{d \nu \lambda} u_{b}{ }^{e}{ }_{\nu} u_{d}{ }^{f}{ }_{\lambda} T_{c e f \mu}+$ H.c. & 140 & 324 \\
\hline $\bar{T}^{a b c \mu} u_{a}{ }^{d \nu} u_{b}{ }^{e \lambda} u_{d}{ }^{f}{ }_{\nu} u_{e}{ }^{g} T_{c f g \mu}$ & 12 & 27 & $i \bar{T}^{a b c \mu} f_{+a}{ }^{d}{ }_{\mu}^{\nu} u^{e f}{ }_{\nu} u_{f e}{ }^{\lambda} T_{b c d \lambda}+$ H.c. & & 325 \\
\hline $\bar{T}^{a b c \mu} u_{a}{ }^{d \nu} u_{b}{ }^{e \lambda} u_{d}{ }_{\lambda}^{f} u_{e}{ }_{\nu}{ }_{\nu} T_{c f g \mu}$ & & 28 & $i \bar{T}^{a b c \mu} f_{+a}{ }^{d \nu \lambda} u_{b}{ }^{e}{ }_{\nu} u_{e}{ }^{f}{ }_{\lambda} T_{c d f \mu}$ & 141 & 326 \\
\hline $\bar{T}^{a b c \mu} u_{a}{ }^{d \nu} u_{b}{ }^{e \lambda} u_{d}{ }_{\lambda}{ }_{\lambda} u_{f}^{g}{ }_{\mu} T_{c e g \nu}$ & & 29 & $i \bar{T}^{a b c \mu} f_{+a}{ }^{d \nu \lambda} u_{d}{ }^{e}{ }_{\mu} u_{e}{ }^{f}{ }_{\nu} T_{b c f \lambda}+$ H.c. & & 327 \\
\hline $\bar{T}^{a b c \mu} u_{a}{ }^{d \nu} u_{b}{ }^{e \lambda} u_{d}{ }_{\lambda}{ }_{\lambda} u_{f}^{g}{ }_{\nu} T_{c e g \mu}$ & & 30 & $i \bar{T}^{a b c \mu} f_{+a \mu}{ }^{d}{ }^{\nu} u^{e f \lambda} u_{f e \lambda} T_{b c d \nu}$ & & 328 \\
\hline
\end{tabular}


TABLE V. (Continued)

\begin{tabular}{|c|c|c|c|c|c|}
\hline$O_{n}^{\left(N_{f}, 4\right)}$ & $S U(2)$ & $S U(3)$ & $O_{n}^{\left(N_{f}, 4\right)}$ & $S U(2)$ & $S U(3)$ \\
\hline $\bar{T}^{a b c \mu} u_{a}{ }^{d \nu} u_{b}{ }^{e}{ }_{\nu} u^{f g \lambda} u_{g f \lambda} T_{c d e \mu}$ & & 31 & $i \bar{T}^{a b c \mu} f_{+a}{ }^{d \nu \lambda} u_{d}{ }^{e}{ }_{\nu} u_{e}{ }_{\mu} T_{b c f \lambda}+$ H.c. & & 329 \\
\hline $\bar{T}^{a b c \mu} u_{a}{ }^{d \nu} u_{d}{ }^{e}{ }_{\mu} u_{e}{ }^{f \lambda} u_{f}{ }_{\nu}^{g} T_{b c g \lambda}$ & & 32 & $i \bar{T}^{a b c \mu} f_{+a}{ }^{d \nu \lambda} u_{d}{ }^{e}{ }_{\nu} u_{e}{ }_{\lambda}^{f} T_{b c f \mu}+$ H.c. & & 330 \\
\hline $\bar{T}^{a b c \mu} u_{a}{ }^{d \nu} u_{d}{ }^{e}{ }_{\mu} u_{e}{ }^{f \lambda} u_{f}{ }_{\lambda}{ }_{\lambda} T_{b c g \nu}+$ H.c. & & 33 & $i \bar{T}^{a b c \mu} f_{+}{ }^{d e}{ }_{\mu}^{\nu} u_{a d \nu} u_{e}^{f \lambda} T_{b c f \lambda}+$ H.c. & & 331 \\
\hline $\bar{T}^{a b c \mu} u_{a}{ }^{d \nu} u_{d}{ }^{e}{ }_{\mu} u^{f g}{ }_{\nu} u_{g f}{ }^{\lambda} T_{b c e \lambda}+$ H.c. & & 34 & $i \bar{T}^{a b c \mu} f_{+}{ }^{d e}{ }_{\mu}{ }^{\nu} u_{a d}{ }^{\lambda} u_{e}{ }^{f}{ }_{\nu} T_{b c f \lambda}+$ H.c. & & 332 \\
\hline $\bar{T}^{a b c \mu} u_{a}{ }^{d \nu} u_{b}{ }^{e \lambda} u^{f g}{ }_{\nu} u_{g f \lambda} T_{c d e \mu}$ & & 35 & $i \bar{T}^{a b c \mu} f_{+}{ }^{\mu}{ }_{\mu}{ }_{\mu}^{\nu} u_{a d}{ }^{\lambda} u_{e}{ }_{\lambda}{ }_{\lambda} T_{b c f \nu}$ & & 333 \\
\hline $\bar{T}^{a b c \mu} u_{a}{ }^{d \nu} u_{d}{ }_{\nu}{ }_{\nu} u^{f g}{ }_{\mu} u_{g f}{ }^{\lambda} T_{b c e \lambda}$ & & 36 & $i \bar{T}^{a b c \mu} f_{+}{ }^{\mu}{ }_{\mu}{ }_{\mu} u_{a}{ }^{f}{ }_{\nu} u_{e d}{ }^{\lambda} T_{b c f \lambda}+$ H.c. & & 334 \\
\hline $\bar{T}^{a b c \mu} u_{a}{ }^{d \nu} u_{d \mu}{ }^{e} u^{f g \lambda} u_{g f \lambda} T_{b c e \nu}$ & & 37 & $i \bar{T}^{a b c \mu} f_{+}{ }^{d e}{ }_{\mu}^{\nu} u_{a}{ }^{f \lambda} u_{e d \nu} T_{b c f \lambda}+$ H.c. & & 335 \\
\hline $\bar{T}^{a b c \mu} u_{a}{ }^{d \nu} u_{d}{ }^{e}{ }_{\nu} u_{e}{ }^{f \lambda} u_{f}{ }_{\lambda}{ }_{\lambda} T_{b c g \mu}$ & & 38 & $i \bar{T}^{a b c \mu} f_{+}{ }^{d e}{ }_{\mu}^{\nu} u_{a}{ }^{f \lambda} u_{e d \lambda} T_{b c f \nu}$ & & 336 \\
\hline $\bar{T}^{a b c \mu} u_{a}{ }^{d \nu} u_{d}{ }^{e \lambda} u_{e}{ }_{\nu}{ }_{\nu} u_{f}^{g}{ }_{\lambda} T_{b c g \mu}$ & & 39 & $i \bar{T}^{a b c \mu} f_{+}{ }^{\operatorname{de\nu } \lambda} u_{a d \nu} u_{e}{ }_{\lambda}^{f} T_{b c f \mu}$ & & 337 \\
\hline $\bar{T}^{a b c \mu} u_{a}{ }^{d \nu} u_{d}{ }^{e}{ }_{\nu} u^{f g \lambda} u_{g f \lambda} T_{b c e \mu}$ & & 40 & $i \bar{T}^{a b c \mu} f_{+}{ }^{d e}{ }_{\mu}{ }^{\nu} u_{e}{ }_{\nu}{ }_{\nu} u_{f d}{ }^{\lambda} T_{a b c \lambda}+$ H.c. & & 338 \\
\hline $\bar{T}^{a b c \mu} u_{a}{ }^{d \nu} u^{e f}{ }_{\mu} u_{f}^{g}{ }_{\nu} u_{g e}{ }^{\lambda} T_{b c d \lambda}$ & & 41 & $i \bar{T}^{a b c \mu} f_{+}{ }^{d e \nu \lambda} u_{e}{ }_{\nu}{ } u_{f d \lambda} T_{a b c \mu}$ & & 339 \\
\hline $\bar{T}^{a b c \mu} u^{d e}{ }_{\mu} u_{e d}{ }^{\nu} u^{f g}{ }_{\nu} u_{g f}{ }^{\lambda} T_{a b c \lambda}$ & & 42 & $i \bar{T}^{a b c \mu} f_{+a}{ }^{d}{ }_{\mu}^{\nu} u_{b}{ }^{e \lambda} u_{c}{ }^{f \rho} D_{\nu \lambda} T_{d e f \rho}+$ H.c. & 142 & 340 \\
\hline $\bar{T}^{a b c \mu} u_{\mu}^{d e} u_{e d}{ }^{\nu} u^{f g \lambda} u_{g f \lambda} T_{a b c \nu}$ & & 43 & $i \bar{T}^{a b c \mu} f_{+a}{ }^{d \mu}{ }_{\mu}^{\nu} u_{b}{ }^{e \lambda} u_{c}{ }^{f \rho} D_{\lambda \rho} T_{d e f \nu}$ & 143 & 341 \\
\hline $\bar{T}^{a b c \mu} u^{d e \nu} u_{e d \nu} u^{f g \lambda} u_{g f \lambda} T_{a b c \mu}$ & & 44 & $i \bar{T}^{a b c \mu} f_{+a}{ }^{d}{ }_{\mu}^{\nu} u_{b}{ }^{e \lambda} u_{d}{ }^{f \rho} D_{\nu \lambda} T_{c e f \rho}+$ H.c. & 144 & 342 \\
\hline $\bar{T}^{a b c \mu} u^{d e \nu} u_{e d}{ }^{\lambda} u^{f g}{ }_{\nu} u_{g f \lambda} T_{a b c \mu}$ & & 45 & $i \bar{T}^{a b c \mu} f_{+a}{ }^{d}{ }_{\mu}^{\nu} u_{b}{ }^{e \lambda} u_{d}{ }^{f \rho} D_{\nu \rho} T_{c e f \lambda}+$ H.c. & 145 & 343 \\
\hline $\bar{T}^{a b c \mu} u_{a}{ }_{\mu}^{d} u_{b}{ }^{e \nu} u_{c}{ }^{f \lambda} u_{d}^{g \rho} D_{\nu \lambda} T_{e f g \rho}$ & 13 & 46 & $i \bar{T}^{a b c \mu} f_{+a}{ }^{d}{ }_{\mu}^{\nu} u_{b}{ }^{e \lambda} u_{d}{ }^{f \rho} D_{\lambda \rho} T_{c e f \nu}+$ H.c. & 146 & 344 \\
\hline $\bar{T}^{a b c \mu} u_{a}{ }^{d}{ }_{\mu} u_{b}{ }^{e \nu} u_{c}{ }^{f \lambda} u_{d}{ }^{g \rho} D_{\nu \rho} T_{e f g \lambda}+$ H.c. & 14 & 47 & $i \bar{T}^{a b c \mu} f_{+a}{ }^{d}{ }_{\mu}^{\nu} u_{b}{ }^{e \lambda} u_{e}{ }^{f \rho} D_{\nu \lambda} T_{c d f \rho}+$ H.c. & 147 & 345 \\
\hline $\bar{T}^{a b c \mu} u_{a}{ }^{d}{ }_{\mu} u_{b}{ }^{e \nu} u_{c}{ }^{f \lambda} u_{e}{ }^{g \rho} D_{\nu \rho} T_{d f g \lambda}$ & 15 & 48 & $i \bar{T}^{a b c \mu} f_{+a}{ }^{d}{ }_{\mu}^{\nu} u_{b}{ }^{e \lambda} u_{e}{ }^{f \rho} D_{\nu \rho} T_{c d f \lambda}+$ H.c. & 148 & 346 \\
\hline $\bar{T}^{a b c \mu} u_{a}{ }^{d}{ }_{\mu} u_{b}{ }^{e \nu} u_{c}{ }^{f \lambda} u_{e}{ }^{g \rho} D_{\lambda \rho} T_{d f g \nu}+$ H.c. & 16 & 49 & $i \bar{T}^{a b c \mu} f_{+a}{ }^{d}{ }_{\mu}^{\nu} u_{b}{ }^{e \lambda} u_{e}{ }^{f \rho} D_{\lambda \rho} T_{c d f \nu}$ & 149 & 347 \\
\hline $\bar{T}^{a b c \mu} u_{a}{ }^{d}{ }_{\mu} u_{b}{ }^{e \nu} u_{d}{ }^{f \lambda} u_{e}{ }^{g \rho} D_{\nu \lambda} T_{c f g \rho}$ & 17 & 50 & $i \bar{T}^{a b c \mu} f_{+a}{ }^{d}{ }_{\mu}^{\nu} u_{d}{ }^{e \lambda} u_{e}{ }^{f \rho} D_{\nu \lambda} T_{b c f \rho}+$ H.c. & & 348 \\
\hline $\bar{T}^{a b c \mu} u_{a}{ }^{d}{ }_{\mu} u_{b}{ }^{e \nu} u_{d}{ }^{f \lambda} u_{e}{ }^{g \rho} D_{\nu \rho} T_{c f g \lambda}$ & & 51 & $i \bar{T}^{a b c \mu} f_{+a}{ }^{d}{ }_{\mu}{ }^{\nu} u_{d}{ }^{e \lambda} u_{e}{ }^{f \rho} D_{\nu \rho} T_{b c f \lambda}+$ H.c. & & 349 \\
\hline $\bar{T}^{a b c \mu} u_{a}{ }^{d}{ }_{\mu} u_{b}{ }^{e \nu} u_{d}{ }^{f \lambda} u_{e}{ }^{g \rho} D_{\lambda \rho} T_{c f g \nu}+$ H.c. & 18 & 52 & $i \bar{T}^{a b c \mu} f_{+a}{ }^{d}{ }_{\mu}^{\nu} u_{d}{ }^{e \lambda} u_{e}{ }^{f \rho} D_{\lambda \rho} T_{b c f \nu}+$ H.c. & & 350 \\
\hline $\bar{T}^{a b c \mu} u_{a}{ }^{d}{ }_{\mu} u_{b}{ }^{e \nu} u_{d}{ }^{f \lambda} u_{f}{ }^{g \rho} D_{\nu \lambda} T_{c e g \rho}$ & & 53 & $i \bar{T}^{a b c \mu} f_{+a}{ }^{d \nu \lambda} u_{b}{ }^{e}{ }_{\mu} u_{d}{ }^{f \rho} D_{\nu \rho} T_{c e f \lambda}+$ H.c. & 150 & 351 \\
\hline $\bar{T}^{a b c \mu} u_{a}{ }^{d}{ }_{\mu} u_{b}{ }^{e \nu} u_{d}{ }^{f \lambda} u_{f}{ }^{g \rho} D_{\nu \rho} T_{c e g \lambda}+$ H.c. & & 54 & $i \bar{T}^{a b c \mu} f_{+a}{ }^{d \nu \lambda} u_{b}{ }^{e}{ }_{\nu} u_{d}{ }^{f \rho} D_{\lambda \rho} T_{c e f \mu}+$ H.c. & 151 & 352 \\
\hline $\bar{T}^{a b c \mu} u_{a}{ }^{d}{ }_{\mu} u_{b}{ }^{e \nu} u_{d}{ }^{f \lambda} u_{f}{ }^{g \rho} D_{\lambda \rho} T_{c e g \nu}+$ H.c. & & 55 & $i \bar{T}^{a b c \mu} f_{+a}{ }^{d \nu \lambda} u_{b}{ }^{e}{ }_{\nu} u_{e}{ }^{f \rho} D_{\lambda \rho} T_{c d f \mu}+$ H.c. & 152 & 353 \\
\hline $\bar{T}^{a b c \mu} u_{a}{ }^{d}{ }_{\mu} u_{b}{ }^{e \nu} u_{e}{ }^{f \lambda} u_{f}{ }^{g \rho} D_{\nu \rho} T_{c d g \lambda}+$ H.c. & & 56 & $i \bar{T}^{a b c \mu} f_{+a}^{d \nu \lambda} u_{b}{ }^{e \rho} u_{d}^{f}{ }_{\mu} D_{\nu \rho} T_{c e f \lambda}+$ H.c. & 153 & 354 \\
\hline $\bar{T}^{a b c \mu} u_{a}{ }^{d}{ }_{\mu}^{\mu} u_{b}{ }^{e \nu} u_{e}{ }^{f \lambda} u_{f}{ }^{g \rho} D_{\lambda \rho} T_{c d g \nu}+$ H.c. & & 57 & $i \bar{T}^{a b c \mu} f_{+a}{ }^{d}{ }_{\mu}^{\nu} u^{e f \lambda} u_{f e}{ }^{\rho} D_{\nu \lambda} T_{b c d \rho}+$ H.c. & & 355 \\
\hline $\bar{T}^{a b c \mu} u_{a}{ }^{d}{ }_{\mu} u_{b}{ }^{e \nu} u^{f g \lambda} u_{g f}{ }^{\rho} D_{\nu \lambda} T_{c d e \rho}+$ H.c. & & 58 & $i \bar{T}^{a b c \mu} f_{+a}{ }^{d}{ }_{\mu}^{\nu} u^{e f \lambda} u_{f e}{ }^{\rho} D_{\lambda \rho} T_{b c d \nu}$ & & 356 \\
\hline $\bar{T}^{a b c \mu} u_{a}{ }^{d}{ }_{\mu} u_{b}{ }^{e \nu} u^{f g \lambda} u_{g f}{ }^{\rho} D_{\lambda \rho} T_{c d e \nu}$ & & 59 & $i \bar{T}^{a b c \mu} f_{+a}{ }^{d \nu \lambda} u_{b}^{e \rho} u_{d}^{f}{ }_{\nu} D_{\lambda \rho} T_{c e f \mu}+$ H.c. & 154 & 357 \\
\hline $\bar{T}^{a b c \mu} u_{a}{ }^{d \nu} u_{b}{ }^{e}{ }_{\nu} u_{c}{ }^{f \lambda} u_{d}{ }^{g \rho} D_{\lambda \rho} T_{e f g \mu}+$ H.c. & 19 & 60 & $i \bar{T}^{a b c \mu} f_{+a}{ }^{d \nu \lambda} u_{d}{ }^{e}{ }_{\mu} u_{e}{ }^{f \rho} D_{\nu \rho} T_{b c f \lambda}+$ H.c. & & 358 \\
\hline $\bar{T}^{a b c \mu} u_{a}{ }^{d \nu} u_{b}{ }^{e}{ }_{\nu} u_{c}{ }^{f \lambda} u_{f}{ }^{g \rho} D_{\lambda \rho} T_{\text {deg } \mu}$ & 20 & 61 & $i \bar{T}^{a b c \mu} f_{+a}{ }^{d \nu \lambda} u_{d}{ }^{e}{ }_{\nu} u_{e}^{f \rho} D_{\lambda \rho} T_{b c f \mu}+$ H.c. & & 359 \\
\hline $\bar{T}^{a b c \mu} u_{a}{ }^{d}{ }_{\mu} u_{d}{ }^{e \nu} u_{e}{ }^{f \lambda} u_{f}{ }^{g \rho} D_{\nu \lambda} T_{b c g \rho}$ & & 62 & $i \bar{T}^{a b c \mu} f_{+a}{ }^{d \nu \lambda} u_{d}^{e \rho} u_{e}{ }_{\mu}^{f} D_{\nu \rho} T_{b c f \lambda}+$ H.c. & & 360 \\
\hline $\bar{T}^{a b c \mu} u_{a}{ }^{d}{ }_{\mu} u_{d}{ }^{e \nu} u_{e}{ }^{f \lambda} u_{f}{ }^{g \rho} D_{\nu \rho} T_{b c g \lambda}+$ H.c. & & 63 & $i \bar{T}^{a b c \mu} f_{+a}{ }^{d \nu \lambda} u_{d}^{e \rho} u_{e}^{f}{ }_{\nu} D_{\lambda \rho} T_{b c f \mu}+$ H.c. & & 361 \\
\hline $\bar{T}^{a b c \mu} u_{a}{ }^{d}{ }_{\mu} u_{d}{ }^{e \nu} u_{e}{ }^{f \lambda} u_{f}{ }^{g \rho} D_{\lambda \rho} T_{b c g \nu}+$ H.c. & & 64 & $i \bar{T}^{a b c \mu} f_{+}{ }^{d e}{ }_{\mu}{ }^{\nu} u_{a d}{ }^{\lambda} u_{e}{ }^{f \rho} D_{\nu \lambda} T_{b c f \rho}+$ H.c. & & 362 \\
\hline $\bar{T}^{a b c \mu} u_{a}{ }^{d \nu} u_{b}{ }_{\nu}{ }_{\nu} u_{d}{ }^{f \lambda} u_{e}{ }^{g \rho} D_{\lambda \rho} T_{c f g \mu}+$ H.c. & 21 & 65 & $i \bar{T}^{a b c \mu} f_{+}{ }^{d e}{ }_{\mu}{ }^{\nu} u_{a d}{ }^{\lambda} u_{e}{ }^{f \rho} D_{\nu \rho} T_{b c f \lambda}+$ H.c. & & 363 \\
\hline $\bar{T}^{a b c \mu} u_{a}{ }^{d \nu} u_{b}{ }_{\nu}{ }_{\nu} u_{d}{ }^{f \lambda} u_{f}{ }^{g \rho} D_{\lambda \rho} T_{c e g \mu}+$ H.c. & & 66 & $i \bar{T}^{a b c \mu} f_{+}{ }^{d e}{ }_{\mu}^{\nu} u_{a d}{ }^{\lambda} u_{e}{ }^{f \rho} D_{\lambda \rho} T_{b c f \nu}$ & & 364 \\
\hline $\bar{T}^{a b c \mu} u_{a}{ }^{d \nu} u_{b}{ }^{e \lambda} u_{d}{ }_{\mu}^{f} u_{e}{ }^{g \rho} D_{\nu \rho} T_{c f g \lambda}$ & 22 & 67 & $i \bar{T}^{a b c \mu} f_{+}{ }^{d e}{ }_{\mu}{ }^{\nu} u_{a}{ }^{f \lambda} u_{e d}{ }^{\rho} D_{\nu \lambda} T_{b c f \rho}+$ H.c. & & 365 \\
\hline $\bar{T}^{a b c \mu} u_{a}{ }^{d \nu} u_{b}{ }^{e \lambda} u_{d}{ }_{\mu}^{f} u_{e}^{g \rho} D_{\lambda \rho} T_{c f g \nu}$ & 23 & 68 & $i \bar{T}^{a b c \mu} f_{+}{ }^{d e}{ }_{\mu}{ }^{\nu} u_{a}{ }^{f \lambda} u_{e d}{ }^{\rho} D_{\nu \rho} T_{b c f \lambda}+$ H.c. & & 366 \\
\hline $\bar{T}^{a b c \mu} u_{a}{ }^{d \nu} u_{b}{ }^{e \lambda} u_{d}{ }^{f}{ }_{\mu} u_{f}^{g \rho} D_{\lambda \rho} T_{c e g \nu}+$ H.c. & & 69 & $i \bar{T}^{a b c \mu} f_{+}{ }^{d e}{ }_{\mu}^{\nu} u_{a}{ }^{f \lambda} u_{e d}{ }^{\rho} D_{\lambda \rho} T_{b c f \nu}$ & & 367 \\
\hline $\bar{T}^{a b c \mu} u_{a}{ }^{d \nu} u_{b}{ }^{e \lambda} u_{c}{ }^{f \rho} u_{d}{ }_{\mu}^{g} D_{\lambda \rho} T_{e f g \nu}$ & & 70 & $i \bar{T}^{a b c \mu} f_{+}{ }^{\operatorname{de\nu } \lambda} u_{a d \nu} u_{e}^{f \rho} D_{\lambda \rho} T_{b c f \mu}+$ H.c. & & 368 \\
\hline $\bar{T}^{a b c \mu} u_{a}{ }^{d \nu} u_{b}{ }^{e \lambda} u_{c}{ }^{f \rho} u_{d}{ }^{g}{ }_{\nu} D_{\lambda \rho} T_{e f g \mu}$ & 24 & 71 & $i \bar{T}^{a b c \mu} f_{+}{ }^{d e}{ }_{\mu}^{\nu} u_{e}{ }^{f \lambda} u_{f d}{ }^{\rho} D_{\nu \lambda} T_{a b c \rho}+$ H.c. & & 369 \\
\hline $\bar{T}^{a b c \mu} u_{a}{ }^{d \nu} u_{b}{ }^{e \lambda} u_{d}{ }_{\nu}^{f} u_{e}{ }^{g \rho} D_{\lambda \rho} T_{c f g \mu}$ & 25 & 72 & $i \bar{T}^{a b c \mu} f_{+}{ }^{\operatorname{de\nu } \lambda} u_{e}{ }^{f}{ }_{\nu} u_{f d}{ }^{\rho} D_{\lambda \rho} T_{a b c \mu}+$ H.c. & & 370 \\
\hline $\bar{T}^{a b c \mu} u_{a}{ }^{d \nu} u_{b}{ }^{e \lambda} u_{d}{ }_{\nu}^{f}{ }_{\nu} u_{f}^{g \rho} D_{\lambda \rho} T_{c e g \mu}+$ H.c. & & 73 & $\bar{T}^{a b c \mu} f_{+a}{ }^{d}{ }_{\mu}^{\nu} u_{b}{ }^{e \lambda} u_{c}{ }^{f \rho} \sigma_{\nu \lambda} T_{d e f \rho}+$ H.c. & 155 & 371 \\
\hline $\bar{T}^{a b c \mu} u_{a}{ }^{d \nu} u_{b}{ }^{e \lambda} u_{d}{ }_{\lambda}^{f} u_{e}{ }^{g \rho} D_{\nu \rho} T_{c f g \mu}$ & & 74 & $\bar{T}^{a b c \mu} f_{+a}{ }^{d}{ }_{\mu}^{\nu} u_{b}{ }^{e \lambda} u_{d}{ }^{f \rho} \sigma_{\nu \lambda} T_{c e f \rho}+$ H.c. & 156 & 372 \\
\hline $\bar{T}^{a b c \mu} u_{a}{ }^{d \nu} u_{b}{ }^{e}{ }_{\nu} u^{f g \lambda} u_{g f}{ }^{\rho} D_{\lambda \rho} T_{c d e \mu}$ & & 75 & $\bar{T}^{a b c \mu} f_{+a}{ }^{d}{ }_{\mu}^{\nu} u_{b}{ }^{e \lambda} u_{d}{ }^{f \rho} \sigma_{\nu \rho} T_{c e f \lambda}+$ H.c. & 157 & 373 \\
\hline $\bar{T}^{a b c \mu} u_{a}{ }^{d \nu} u_{b}{ }^{e \lambda} u_{d}{ }_{\lambda}{ }_{\lambda} u_{f}^{g \rho} D_{\nu \rho} T_{c e g \mu}$ & & 76 & $\bar{T}^{a b c \mu} f_{+a}{ }^{d}{ }_{\mu}^{\nu} u_{b}{ }^{e \lambda} u_{e}{ }^{f \rho} \sigma_{\nu \lambda} T_{c d f \rho}+$ H.c. & 158 & 374 \\
\hline $\bar{T}^{a b c \mu} u_{a}{ }^{d \nu} u_{b}{ }^{e \lambda} u_{d}{ }^{f \rho} u_{f}^{g}{ }_{\mu} D_{\lambda \rho} T_{c e g \nu}$ & & 77 & $\bar{T}^{a b c \mu} f_{+a}{ }^{d}{ }_{\mu}^{\nu} u_{b}{ }^{e \lambda} u_{e}{ }^{f \rho} \sigma_{\nu \rho} T_{c d f \lambda}+$ H.c. & 159 & 375 \\
\hline
\end{tabular}


TABLE V. (Continued)

\begin{tabular}{|c|c|c|c|c|c|}
\hline$O_{n}^{\left(N_{f}, 4\right)}$ & $S U(2)$ & $S U(3)$ & $O_{n}^{\left(N_{f}, 4\right)}$ & $S U(2)$ & $S U(3)$ \\
\hline $\bar{T}^{a b c \mu} u_{a}{ }^{d \nu} u_{b}{ }^{e \lambda} u_{d}{ }^{f \rho} u_{f}{ }^{g}{ }_{\nu} D_{\lambda \rho} T_{c e g \mu}$ & & 78 & $i \bar{T}^{a b c \mu} f_{s,+\mu}{ }^{\nu} u_{a}{ }^{d}{ }_{\nu} u_{b}{ }^{e \lambda} T_{c d e \lambda}+$ H.c. & 160 & \\
\hline $\bar{T}^{a b c \mu} u_{a}{ }^{d \nu} u_{d}{ }^{e}{ }_{\mu} u_{e}{ }^{f \lambda} u_{f}{ }^{g \rho} D_{\nu \rho} T_{b c g \lambda}$ & & 79 & $i \bar{T}^{a b c \mu} f_{s,+\mu}{ }^{\nu} u_{a}{ }^{d \lambda} u_{b}{ }^{e}{ }_{\lambda} T_{c d e \nu}$ & 161 & \\
\hline $\bar{T}^{a b c \mu} u_{a}{ }^{d \nu} u_{d}{ }^{e}{ }_{\mu} u_{e}{ }^{f \lambda} u_{f}{ }^{g \rho} D_{\lambda \rho} T_{b c g \nu}+$ H.c. & & 80 & $i \bar{T}^{a b c \mu} f_{s,+\mu}{ }^{\nu} u_{a}{ }^{d}{ }_{\nu} u_{d}{ }^{e \lambda} T_{b c e \lambda}+$ H.c. & 162 & \\
\hline $\bar{T}^{a b c \mu} u_{a}{ }^{d \nu} u_{b}{ }^{e \lambda} u^{f g}{ }_{\mu} u_{g f}{ }^{\rho} D_{\nu \lambda} T_{c d e \rho}$ & & 81 & $i \bar{T}^{a b c \mu} f_{s,+\mu}{ }^{\nu} u_{a}{ }^{d \lambda} u_{d}{ }^{e}{ }_{\nu} T_{b c e \lambda}+$ H.c. & 163 & \\
\hline $\bar{T}^{a b c \mu} u_{a}{ }^{d \nu} u_{b}{ }^{e \lambda} u^{f g}{ }_{\nu} u_{g f} \rho D_{\lambda \rho} T_{c d e \mu}$ & & 82 & $i \bar{T}^{a b c \mu} f_{s,+\mu}{ }^{\nu} u_{a}{ }^{d \lambda} u_{d}{ }^{e}{ }_{\lambda} T_{b c e \nu}$ & 164 & \\
\hline $\bar{T}^{a b c \mu} u_{a}{ }^{d \nu} u_{d \mu}{ }^{e} u^{f g \lambda} u_{g f}{ }^{\rho} D_{\nu \lambda} T_{b c e \rho}+$ H.c. & & 83 & $i \bar{T}^{a b c \mu} f_{s,+}{ }^{\nu \lambda} u_{a}{ }^{d}{ }_{\nu} u_{d}{ }^{e} T_{b c e \mu}$ & 165 & \\
\hline $\bar{T}^{a b c \mu} u_{a}{ }^{d \nu} u_{d \mu}^{e} u^{f g \lambda} u_{g f}{ }^{\rho} D_{\lambda \rho} T_{b c e \nu}$ & & 84 & $i \bar{T}^{a b c \mu} f_{s,+\mu}{ }^{\nu} u_{a}{ }^{d \lambda} u_{b}{ }^{e \rho} D_{\nu \lambda} T_{c d e \rho}+$ H.c. & 166 & \\
\hline $\bar{T}^{a b c \mu} u_{a}{ }^{d \nu} u_{d}{ }^{e}{ }_{\nu} u_{e}{ }^{f \lambda} u_{f}^{g \rho} D_{\lambda \rho} T_{b c g \mu}+$ H.c. & & 85 & $i \bar{T}^{a b c \mu} f_{s,+\mu}{ }^{\nu} u_{a}^{d \lambda} u_{b}^{e \rho} D_{\lambda \rho} T_{c d e \nu}$ & 167 & \\
\hline $\bar{T}^{a b c \mu} u_{a}{ }^{d \nu} u_{d}{ }^{e \lambda} u_{e}{ }_{\mu}^{f} u_{f}^{g \rho} D_{\nu \rho} T_{b c g \lambda}$ & & 86 & $i \bar{T}^{a b c \mu} f_{s,+\mu}{ }^{\nu} u_{a}^{d \lambda} u_{d}^{e \rho} D_{\nu \lambda} T_{b c e \rho}+$ H.c. & 168 & \\
\hline $\bar{T}^{a b c \mu} u_{a}{ }^{d \nu} u_{b}{ }^{e \lambda} u^{f g \rho} u_{g f \rho} D_{\nu \lambda} T_{c d e \mu}$ & & 87 & $i \bar{T}^{a b c \mu} f_{s,+\mu}{ }^{\nu} u_{a}^{d \lambda} u_{d}^{e \rho} D_{\nu \rho} T_{b c e \lambda}+$ H.c. & 169 & \\
\hline $\bar{T}^{a b c \mu} u_{a}{ }^{d \nu} u_{d}{ }^{e}{ }_{\nu} u^{f g \lambda} u_{g f}{ }^{\rho} D_{\lambda \rho} T_{b c e \mu}$ & & 88 & $i \bar{T}^{a b c \mu} f_{s,+\mu}{ }^{\nu} u_{a}{ }^{d \lambda} u_{d}^{e \rho} D_{\lambda \rho} T_{b c e \nu}$ & 170 & \\
\hline $\bar{T}^{a b c \mu} u_{a}{ }^{d \nu} u_{d}{ }^{e \lambda} u_{e}{ }^{f}{ }_{\nu} u_{f}{ }^{g \rho} D_{\lambda \rho} T_{b c g \mu}+$ H.c. & & 89 & $i \bar{T}^{a b c \mu} f_{s,+}{ }^{\nu \lambda} u_{a}{ }^{d}{ }_{\nu} u_{d}{ }^{e \rho} D_{\lambda \rho} T_{b c e \mu}+$ H.c. & 171 & \\
\hline $\bar{T}^{a b c \mu} u_{a}{ }^{d \nu} u_{d}{ }^{e \lambda} u_{e}{ }_{\lambda}^{f} u_{f}^{g \rho} D_{\nu \rho} T_{b c g \mu}$ & & 90 & $\bar{T}^{a b c \mu} f_{s,+\mu}{ }^{\nu} u_{a}{ }^{d \lambda} u_{b}{ }^{e \rho} \sigma_{\nu \lambda} T_{c d e \rho}+$ H.c. & 172 & \\
\hline $\bar{T}^{a b c \mu} u_{a}{ }^{d \nu} u^{e f}{ }_{\mu} u_{f}^{g \lambda} u_{g e}{ }^{\rho} D_{\nu \lambda} T_{b c d \rho}$ & & 91 & $\bar{T}^{a b c \mu} f_{s,+\mu}{ }^{\nu} u_{a}{ }^{d \lambda} u_{d}{ }^{e \rho} \sigma_{\nu \lambda} T_{b c e \rho}+$ H.c. & 173 & \\
\hline $\bar{T}^{a b c \mu} u^{d e}{ }_{\mu} u_{e d}{ }^{\nu} u^{f g \lambda} u_{g f}{ }^{\rho} D_{\nu \lambda} T_{a b c \rho}$ & & 92 & $\bar{T}^{a b c \mu} f_{s,+\mu}{ }^{\nu} u_{a}{ }^{d \lambda} u_{d}{ }^{e \rho} \sigma_{\nu \rho} T_{b c e \lambda}+$ H.c. & 174 & \\
\hline $\bar{T}^{a b c \mu} u^{d e}{ }_{\mu} u_{e d}{ }^{\nu} u^{f g \lambda} u_{g f}{ }^{\rho} D_{\lambda \rho} T_{a b c \nu}$ & & 93 & $\bar{T}^{a b c \mu} f_{+a}{ }^{d}{ }_{\mu}{ }^{\nu} u_{d}{ }^{e \lambda} u_{e}{ }^{f \rho} \sigma_{\nu \lambda} T_{b c f \rho}+$ H.c. & & 376 \\
\hline $\bar{T}^{a b c \mu} u^{d e \nu} u_{e d \nu} u^{f g \lambda} u_{g f}{ }^{\rho} D_{\lambda \rho} T_{a b c \mu}$ & & 94 & $\bar{T}^{a b c \mu} f_{+a}{ }^{d}{ }_{\mu}{ }^{\nu} u_{d}{ }^{e \lambda} u_{e}{ }^{f \rho} \sigma_{\nu \rho} T_{b c f \lambda}+$ H.c. & & 377 \\
\hline $\bar{T}^{a b c \mu} u^{d e \nu} u_{e d}{ }^{\lambda} u^{f g}{ }_{\nu} u_{g f}{ }^{\rho} D_{\lambda \rho} T_{a b c \mu}$ & & 95 & $\bar{T}^{a b c \mu} f_{+a}{ }^{d}{ }_{\mu}{ }^{\nu} u^{e f \lambda} u_{f e}{ }^{\rho} \sigma_{\nu \lambda} T_{b c d \rho}+$ H.c. & & 378 \\
\hline $\bar{T}^{a b c \mu} u_{a}{ }^{d \nu} u_{b}{ }^{e \lambda} u_{c}{ }^{f \rho} u_{d}{ }^{g \sigma} D_{\nu \lambda \rho \sigma} T_{e f g \mu}$ & 26 & 96 & $\bar{T}^{a b c \mu} f_{+}{ }^{d e}{ }_{\mu}^{\nu} u_{a d}{ }^{\lambda} u_{e}{ }^{f \rho} \sigma_{\nu \lambda} T_{b c f \rho}+$ H.c. & & 379 \\
\hline $\bar{T}^{a b c \mu} u_{a}{ }^{d \nu} u_{b}{ }^{e \lambda} u_{d}{ }^{f \rho} u_{e}{ }^{g \sigma} D_{\nu \lambda \rho \sigma} T_{c f g \mu}$ & 27 & 97 & $\bar{T}^{a b c \mu} f_{+}{ }^{d e}{ }_{\mu}^{\nu} u_{a d}{ }^{\lambda} u_{e}{ }^{f \rho} \sigma_{\nu \rho} T_{b c f \lambda}+$ H.c. & & 380 \\
\hline $\bar{T}^{a b c \mu} u_{a}{ }^{d \nu} u_{b}{ }^{e \lambda} u_{d}{ }^{f \rho} u_{f}{ }^{g \sigma} D_{\nu \lambda \rho \sigma} T_{c e g \mu}$ & & 98 & $\bar{T}^{a b c \mu} f_{+}{ }^{d e}{ }_{\mu}^{\nu} u_{a}{ }^{f \lambda} u_{e d}{ }^{\rho} \sigma_{\nu \lambda} T_{b c f \rho}+$ H.c. & & 381 \\
\hline $\bar{T}^{a b c \mu} u_{a}{ }^{d \nu} u_{b}{ }^{e \lambda} u^{f g \rho} u_{g f}{ }^{\sigma} D_{\nu \lambda \rho \sigma} T_{c d e \mu}$ & & 99 & $\bar{T}^{a b c \mu} f_{+}{ }^{d e}{ }_{\mu}^{\nu} u_{e}{ }^{f \lambda} u_{f d}{ }^{\rho} \sigma_{\nu \lambda} T_{a b c \rho}+$ H.c. & & 382 \\
\hline $\bar{T}^{a b c \mu} u_{a}{ }^{d \nu} u_{d}{ }^{e \lambda} u_{e}{ }^{f \rho} u_{f}^{g \sigma} D_{\nu \lambda \rho \sigma} T_{b c g \mu}$ & & 100 & $i \bar{T}^{a b c \mu} f_{+a}{ }^{d}{ }_{\mu}^{\nu} f_{-b}{ }^{e \lambda \rho} \gamma_{5} \gamma_{\nu} D_{\lambda} T_{c d e \rho}+$ H.c. & 175 & 383 \\
\hline $\bar{T}^{a b c \mu} u^{d e \nu} u_{e d}^{\lambda} u^{f g \rho} u_{g f}^{\sigma} D_{\nu \lambda \rho \sigma} T_{a b c \mu}$ & & 101 & $i \bar{T}^{a b c \mu} f_{+a}{ }^{d}{ }_{\mu}^{\nu} f_{-b}{ }^{e \lambda \rho} \gamma_{5} \gamma_{\lambda} D_{\nu} T_{c d e \rho}+$ H.c. & 176 & 384 \\
\hline$i \bar{T}^{a b c \mu} u_{a}{ }^{d}{ }_{\mu} u_{b}{ }^{e \nu} u_{c}{ }^{f \lambda} u_{d}{ }^{g \rho} \sigma_{\nu \rho} T_{e f g \lambda}+$ H.c. & 28 & 102 & $i \bar{T}^{a b c \mu} f_{+a}{ }^{d \nu \lambda} f_{-b}{ }^{e}{ }_{\nu} \gamma_{5} \gamma_{\lambda} D_{\rho} T_{c d e \mu}$ & 177 & 385 \\
\hline$i \bar{T}^{a b c \mu} u_{a}{ }^{d} u_{b}{ }^{e \nu} u_{c}{ }^{f \lambda} u_{e}{ }^{g \rho} \sigma_{\nu \rho} T_{d f g \lambda}$ & 29 & 103 & $i \bar{T}^{a b c \mu} f_{+a}{ }^{d \nu \lambda} f_{-b}{ }^{e}{ }_{\nu} \gamma_{5} \gamma_{\rho} D_{\lambda} T_{c d e \mu}$ & 178 & 386 \\
\hline$i \bar{T}^{a b c \mu} u_{a}{ }^{d}{ }_{\mu} u_{b}{ }^{e \nu} u_{d}{ }^{f \lambda} u_{e}{ }^{g \rho} \sigma_{\nu \lambda} T_{c f g \rho}$ & 30 & 104 & $i \bar{T}^{a b c \mu} f_{+a}{ }^{d}{ }_{\mu}^{\nu} f_{-d}{ }^{e \lambda \rho} \gamma_{5} \gamma_{\nu} D_{\lambda} T_{b c e \rho}+$ H.c. & 179 & 387 \\
\hline$i \bar{T}^{a b c \mu} u_{a}^{d}{ }_{\mu}^{d} u_{b}{ }^{e \nu} u_{d}{ }^{f \lambda} u_{e}{ }^{g \rho} \sigma_{\nu \rho} T_{c f g \lambda}$ & & 105 & $i \bar{T}^{a b c \mu} f_{+a}{ }^{d}{ }_{\mu}^{\nu} f_{-d}^{e \lambda \rho} \gamma_{5} \gamma_{\lambda} D_{\nu} T_{b c e \rho}+$ H.c. & 180 & 388 \\
\hline$i \bar{T}^{a b c \mu} u_{a}{ }^{d} u_{b}{ }^{e \nu} u_{d}{ }^{f \lambda} u_{f}{ }^{g \rho} \sigma_{\nu \rho} T_{c e g \lambda}+$ H.c. & & 106 & $i \bar{T}^{a b c \mu} f_{+a}{ }^{d}{ }_{\mu}^{\nu} f_{-d}^{e \lambda \rho} \gamma_{5} \gamma_{\lambda} D_{\rho} T_{b c e \nu}+$ H.c. & 181 & 389 \\
\hline$i \bar{T}^{a b c \mu} u_{a}{ }^{d} u_{b}{ }^{e \nu} u_{e}{ }^{f \lambda} u_{f}{ }^{g \rho} \sigma_{\nu \rho} T_{c d g \lambda}+$ H.c. & & 107 & $i \bar{T}^{a b c \mu} f_{+a}^{d \nu \lambda} f_{-d}{ }^{e}{ }^{\rho} \gamma_{5} \gamma_{\nu} D_{\lambda} T_{b c e \rho}+$ H.c. & 182 & 390 \\
\hline$i \bar{T}^{a b c \mu} u_{a}{ }^{d}{ }_{\mu} u_{b}{ }^{e \nu} u^{f g \lambda} u_{g f}{ }^{\rho} \sigma_{\nu \lambda} T_{c d e \rho}+$ H.c. & & 108 & $i \bar{T}^{a b c \mu} f_{+a}{ }^{d \nu \lambda} f_{-d}{ }^{e}{ }^{\rho} \gamma_{5} \gamma_{\nu} D_{\rho} T_{b c e \lambda}+$ H.c. & 183 & 391 \\
\hline$i \bar{T}^{a b c \mu} u_{a}^{d \nu} u_{b}^{e \lambda} u_{d}^{f}{ }_{\mu} u_{e}^{g \rho} \sigma_{\nu \rho} T_{c f g \lambda}$ & 31 & 109 & $i \bar{T}^{a b c \mu} f_{+a}{ }^{d \nu \lambda} f_{-d}{ }^{e}{ }^{\rho} \gamma_{5} \gamma_{\rho} D_{\nu} T_{b c e \lambda}+$ H.c. & 184 & 392 \\
\hline$i \bar{T}^{a b c \mu} u_{a}{ }^{d}{ }_{\mu} u_{d}^{e \nu} u_{e}^{f \lambda} u_{f}^{g \rho} \sigma_{\nu \lambda} T_{b c g \rho}$ & & 110 & $i \bar{T}^{a b c \mu} f_{+a}{ }^{d \nu \lambda} f_{-d}{ }^{e}{ }_{\nu}^{\rho} \gamma_{5} \gamma_{\lambda} D_{\rho} T_{b c e \mu}+$ H.c. & 185 & 393 \\
\hline$i \bar{T}^{a b c \mu} u_{a}{ }^{d}{ }_{\mu} u_{d}{ }^{e \nu} u_{e}{ }^{f \lambda} u_{f}{ }^{g \rho} \sigma_{\nu \rho} T_{b c g \lambda}+$ H.c. & & 111 & $i \bar{T}^{a b c \mu} f_{+a}^{d \nu \lambda} f_{-d}{ }^{e}{ }_{\nu}^{\rho} \gamma_{5} \gamma_{\rho} D_{\lambda} T_{b c e \mu}+$ H.c. & 186 & 394 \\
\hline$i \bar{T}^{a b c \mu} u_{a}{ }^{d \nu} u_{d}^{e}{ }_{\mu} u_{e}^{f \lambda} u_{f}^{g \rho} \sigma_{\nu \rho} T_{b c g \lambda}$ & & 112 & $i \bar{T}^{a b c \mu} f_{s,+\mu}^{\nu} f_{-a}^{d \lambda \rho} \gamma_{5} \gamma_{\nu} D_{\lambda} T_{b c d \rho}+$ H.c. & 187 & \\
\hline$i \bar{T}^{a b c \mu} u_{a}{ }^{d \nu} u_{d}{ }^{e}{ }_{\mu} u^{f g \lambda} u_{g f}{ }^{\rho} \sigma_{\nu \lambda} T_{b c e \rho}+$ H.c. & & 113 & $i \bar{T}^{a b c \mu} f_{s,+\mu}^{\nu} f_{-a}^{d \lambda \rho} \gamma_{5} \gamma_{\lambda} D_{\nu} T_{b c d \rho}+$ H.c. & 188 & \\
\hline$i \bar{T}^{a b c \mu} u^{d e}{ }_{\mu} u_{e d}{ }^{\nu} u^{f g \lambda} u_{g f}{ }^{\rho} \sigma_{\nu \lambda} T_{a b c \rho}$ & & 114 & $i \bar{T}^{a b c \mu} f_{s,+}{ }^{\nu \lambda} f_{-a}{ }^{d}{ }^{\rho} \gamma_{5} \gamma_{\lambda} D_{\rho} T_{b c d \mu}$ & 189 & \\
\hline $\bar{T}^{a b c \mu} u_{a}{ }^{d}{ }_{\mu} u_{b}{ }^{e \nu} f_{-c}{ }^{f \lambda \rho} \gamma_{5} \gamma_{\nu} D_{\lambda} T_{\text {def } \rho}+$ H.c. & 32 & 115 & $i \bar{T}^{a b c \mu} f_{s,+}{ }^{\nu \lambda} f_{-a}{ }^{d}{ }_{\nu} \gamma_{5} \gamma_{\rho} D_{\lambda} T_{b c d \mu}$ & 190 & \\
\hline $\bar{T}^{a b c \mu} u_{a}{ }^{d}{ }_{\mu} u_{b}{ }^{e \nu} f_{-c}{ }^{f \lambda \rho} \gamma_{5} \gamma_{\lambda} D_{\nu} T_{\text {def } \rho}+$ H.c. & 33 & 116 & $i \bar{T}^{a b c \mu} f_{+}{ }^{d e}{ }_{\mu}^{\nu} f_{-e d}{ }^{\lambda \rho} \gamma_{5} \gamma_{\nu} D_{\lambda} T_{a b c \rho}+$ H.c. & & 395 \\
\hline $\bar{T}^{a b c \mu} u_{a}{ }^{d}{ }_{\mu} u_{b}{ }^{e \nu} f_{-d}{ }^{f \lambda \rho} \gamma_{5} \gamma_{\nu} D_{\lambda} T_{c e f \rho}+$ H.c. & 34 & 117 & $i \bar{T}^{a b c \mu} f_{+}{ }^{d e}{ }_{\mu}^{\nu} f_{-e d}{ }^{\lambda \rho} \gamma_{5} \gamma_{\lambda} D_{\nu} T_{a b c \rho}+$ H.c. & & 396 \\
\hline $\bar{T}^{a b c \mu} u_{a}{ }^{d}{ }_{\mu} u_{b}{ }^{e \nu} f_{-d}{ }^{f \lambda \rho} \gamma_{5} \gamma_{\lambda} D_{\nu} T_{c e f \rho}+$ H.c. & 35 & 118 & $i \bar{T}^{a b c \mu} f_{+}{ }^{d e \nu \lambda} f_{-e d \nu}{ }^{\rho} \gamma_{5} \gamma_{\lambda} D_{\rho} T_{a b c \mu}$ & & 397 \\
\hline $\bar{T}^{a b c \mu} u_{a}{ }_{\mu}^{d} u_{b}{ }^{e \nu} f_{-d}{ }^{f \lambda \rho} \gamma_{5} \gamma_{\lambda} D_{\rho} T_{c e f \nu}+$ H.c. & 36 & 119 & $i \bar{T}^{a b c \mu} f_{+}{ }^{d e \nu \lambda} f_{-e d \nu}{ }^{\rho} \gamma_{5} \gamma_{\rho} D_{\lambda} T_{a b c \mu}$ & & 398 \\
\hline $\bar{T}^{a b c \mu} u_{a}{ }^{d}{ }_{\mu} u_{b}{ }^{e \nu} f_{-e}{ }^{f \lambda \rho} \gamma_{5} \gamma_{\nu} D_{\lambda} T_{c d f \rho}+$ H.c. & 37 & 120 & $i \bar{T}^{a b c \mu} f_{+a{ }_{\mu}{ }^{\nu} h_{b}{ }^{e \lambda \rho} \gamma_{5} \gamma_{\nu} D_{\lambda} T_{c d e \rho}+\text { H.c. }}$ & 191 & 399 \\
\hline $\bar{T}^{a b c \mu} u_{a}{ }^{d}{ }_{\mu} u_{b}{ }^{e \nu} f_{-e}{ }^{f \lambda \rho} \gamma_{5} \gamma_{\lambda} D_{\nu} T_{c d f \rho}+$ H.c. & 38 & 121 & $i \bar{T}^{a b c \mu} f_{+a{ }_{\mu}{ }^{\nu} h_{b}{ }^{e \lambda \rho} \gamma_{5} \gamma_{\lambda} D_{\nu} T_{c d e \rho}+\text { H.c. }}$ & 192 & 400 \\
\hline $\bar{T}^{a b c \mu} u_{a}{ }^{d}{ }_{\mu} u_{b}{ }^{e \nu} f_{-e}{ }^{f \lambda \rho} \gamma_{5} \gamma_{\lambda} D_{\rho} T_{c d f \nu}+$ H.c. & 39 & 122 & $i \bar{T}^{a b c \mu} f_{+a}{ }^{d \nu \lambda} h_{b}{ }^{e}{ }_{\mu}^{\rho} \gamma_{5} \gamma_{\nu} D_{\lambda} T_{c d e \rho}$ & 193 & 401 \\
\hline $\bar{T}^{a b c \mu} u_{a}{ }^{d}{ }_{\mu} u_{d}{ }^{e \nu} f_{-b}{ }^{f \lambda \rho} \gamma_{5} \gamma_{\nu} D_{\lambda} T_{c e f \rho}+$ H.c. & 40 & 123 & $i \bar{T}^{a b c \mu} f_{+a}{ }^{d \nu \lambda} h_{b}{ }^{e}{ }_{\nu} \gamma_{5} \gamma_{\lambda} D_{\rho} T_{c d e \mu}$ & 194 & 402 \\
\hline $\bar{T}^{a b c \mu} u_{a}{ }^{d}{ }_{\mu} u_{d}{ }^{e \nu} f_{-b}{ }^{f \lambda \rho} \gamma_{5} \gamma_{\lambda} D_{\nu} T_{c e f \rho}+$ H.c. & 41 & 124 & $i \bar{T}^{a b c \mu} f_{+a}{ }^{d \nu \lambda} h_{b}{ }^{e}{ }_{\nu}{ }^{\rho} \gamma_{5} \gamma_{\rho} D_{\lambda} T_{c d e \mu}$ & 195 & 403 \\
\hline $\bar{T}^{a b c \mu} u_{a}^{d \nu} u_{b}^{e \lambda} f_{-c}^{f}{ }_{\mu}^{\rho} \gamma_{5} \gamma_{\nu} D_{\lambda} T_{\text {def } \rho}$ & 42 & 125 & $i \bar{T}^{a b c \mu} f_{+a}{ }^{d}{ }_{\mu}^{\nu} h_{d}{ }^{e \lambda \rho} \gamma_{5} \gamma_{\nu} D_{\lambda} T_{b c e \rho}+$ H.c. & 196 & 404 \\
\hline
\end{tabular}


TABLE V. (Continued)

\begin{tabular}{|c|c|c|c|c|c|}
\hline$O_{n}^{\left(N_{f}, 4\right)}$ & $S U(2)$ & $S U(3)$ & $O_{n}^{\left(N_{f}, 4\right)}$ & $S U(2)$ & $S U(3)$ \\
\hline $\bar{T}^{a b c \mu} u_{a}{ }^{d}{ }_{\mu} u_{d}{ }^{e \nu} f_{-e}{ }^{f \lambda \rho} \gamma_{5} \gamma_{\nu} D_{\lambda} T_{b c f \rho}+$ H.c. & & 126 & $i \bar{T}^{a b c \mu} f_{+a}{ }^{d}{ }_{\mu}^{\nu} h_{d}{ }^{e \lambda \rho} \gamma_{5} \gamma_{\lambda} D_{\nu} T_{b c e \rho}+$ H.c. & 197 & 405 \\
\hline $\bar{T}^{a b c \mu} u_{a}{ }^{d}{ }_{\mu} u_{d}{ }^{e \nu} f_{-e}{ }^{f \lambda \rho} \gamma_{5} \gamma_{\lambda} D_{\nu} T_{b c f \rho}+$ H.c. & & 127 & $i \bar{T}^{a b c \mu} f_{+a}{ }^{d \mu}{ }_{\mu}^{\nu} h_{d}{ }^{e \lambda \rho} \gamma_{5} \gamma_{\lambda} D_{\rho} T_{b c e \nu}+$ H.c. & 198 & 406 \\
\hline $\bar{T}^{a b c \mu} u_{a}{ }^{d}{ }_{\mu} u_{d}{ }^{e \nu} f_{-e}{ }^{f \lambda \rho} \gamma_{5} \gamma_{\lambda} D_{\rho} T_{b c f \nu}+$ H.c. & & 128 & $i \bar{T}^{a b c \mu} f_{+a}^{d \nu \lambda} h_{d \mu}^{e}{ }^{\rho} \gamma_{5} \gamma_{\nu} D_{\lambda} T_{b c e \rho}+$ H.c. & 199 & 407 \\
\hline $\bar{T}^{a b c \mu} u_{a}{ }^{d \nu} u_{b}{ }^{e \lambda} f_{-d}{ }^{f}{ }_{\mu}^{\rho} \gamma_{5} \gamma_{\nu} D_{\lambda} T_{c e f \rho}+$ H.c. & 43 & 129 & $i \bar{T}^{a b c \mu} f_{+a}^{d \nu \lambda} h_{d} e_{\mu}^{\mu} \gamma_{5} \gamma_{\nu} D_{\rho} T_{b c e \lambda}+$ H.c. & 200 & 408 \\
\hline $\bar{T}^{a b c \mu} u_{a}{ }^{d \nu} u_{b}{ }^{e \lambda} f_{-d}{ }^{\mu}{ }_{\mu}^{\rho} \gamma_{5} \gamma_{\nu} D_{\rho} T_{c e f \lambda}+$ H.c. & 44 & 130 & $i \bar{T}^{a b c \mu} f_{+a} d \nu \lambda h_{d}^{e}{ }_{\nu}^{\rho} \gamma_{5} \gamma_{\lambda} D_{\rho} T_{b c e \mu}+$ H.c. & 201 & 409 \\
\hline $\bar{T}^{a b c \mu} u_{a}{ }^{d \nu} u_{d}{ }_{\mu} f_{-b}{ }^{f \lambda \rho} \gamma_{5} \gamma_{\nu} D_{\lambda} T_{c e f \rho}+$ H.c. & 45 & 131 & $i \bar{T}^{a b c \mu} f_{+a}{ }^{d \nu \lambda} h_{d}^{e}{ }_{\nu}^{\rho} \gamma_{5} \gamma_{\rho} D_{\lambda} T_{b c e \mu}+$ H.c. & 202 & 410 \\
\hline $\bar{T}^{a b c \mu} u_{a}{ }^{d \nu} u_{b}{ }^{e}{ }_{\nu} f_{-d}{ }^{f \lambda \rho} \gamma_{5} \gamma_{\lambda} D_{\rho} T_{c e f \mu}+$ H.c. & 46 & 132 & $i \bar{T}^{a b c \mu} f_{+}{ }^{d e}{ }_{\mu}{ }^{\nu} h_{e d}{ }^{\lambda \rho} \gamma_{5} \gamma_{\nu} D_{\lambda} T_{a b c \rho}+$ H.c. & & 411 \\
\hline $\bar{T}^{a b c \mu} u_{a}{ }^{d}{ }_{\mu} u^{e f \nu} f_{-b e}{ }^{\lambda \rho} \gamma_{5} \gamma_{\lambda} D_{\nu} T_{c d f \rho}+$ H.c. & 47 & 133 & $i \bar{T}^{a b c \mu} f_{+}{ }^{d e}{ }_{\mu}{ }^{\nu} h_{e d}{ }^{\lambda \rho} \gamma_{5} \gamma_{\lambda} D_{\nu} T_{a b c \rho}+$ H.c. & & 412 \\
\hline $\bar{T}^{a b c \mu} u_{a}{ }^{d \nu} u_{b}{ }^{e \lambda} f_{-d}^{f}{ }_{\mu}^{\rho} \gamma_{5} \gamma_{\lambda} D_{\nu} T_{c e f \rho}+$ H.c. & 48 & 134 & $i \bar{T}^{a b c \mu} f_{+}{ }^{d e \nu \lambda} h_{e d \mu}{ }^{\rho} \gamma_{5} \gamma_{\nu} D_{\lambda} T_{a b c \rho}$ & & 413 \\
\hline $\bar{T}^{a b c \mu} u_{a}{ }^{d \nu} u_{b}{ }^{e \lambda} f_{-d}^{f}{ }_{\mu}^{\rho} \gamma_{5} \gamma_{\lambda} D_{\rho} T_{c e f \nu}+$ H.c. & 49 & 135 & $i \bar{T}^{a b c \mu} f_{+}{ }^{d e \nu \lambda} h_{e d \nu}{ }^{\rho} \gamma_{5} \gamma_{\lambda} D_{\rho} T_{a b c \mu}$ & & 414 \\
\hline $\bar{T}^{a b c \mu} u_{a}{ }^{d \nu} u_{d}{ }^{e} f_{-b}{ }^{f \lambda \rho} \gamma_{5} \gamma_{\lambda} D_{\nu} T_{c e f \rho}+$ H.c. & 50 & 136 & $i \bar{T}^{a b c \mu} f_{+}{ }^{\operatorname{de\nu } \lambda} h_{e d \nu}{ }^{\rho} \gamma_{5} \gamma_{\rho} D_{\lambda} T_{a b c \mu}$ & & 415 \\
\hline $\bar{T}^{a b c \mu} u_{a}{ }^{d \nu} u_{b}{ }^{e \lambda} f_{-d}^{f}{ }_{\mu}^{\rho} \gamma_{5} \gamma_{\rho} D_{\lambda} T_{c e f \nu}+$ H.c. & 51 & 137 & $i \bar{T}^{a b c \mu} f_{+a}{ }^{d \nu \lambda} h_{b}{ }^{e \rho \sigma} \gamma_{5} \gamma_{\nu} D_{\lambda \rho \sigma} T_{c d e \mu}$ & 203 & 416 \\
\hline $\bar{T}^{a b c \mu} u_{a}{ }^{d \nu} u_{b}{ }^{e \lambda} f_{-d}{ }^{f}{ }_{\nu}{ }^{\rho} \gamma_{5} \gamma_{\lambda} D_{\rho} T_{c e f \mu}+$ H.c. & 52 & 138 & $i \bar{T}^{a b c \mu} f_{+a}{ }^{d \nu \lambda} h_{d}{ }^{e \rho \sigma} \gamma_{5} \gamma_{\nu} D_{\lambda \rho \sigma} T_{b c e \mu}+$ H.c. & 204 & 417 \\
\hline $\bar{T}^{a b c \mu} u_{a}{ }^{d}{ }_{\mu} u^{e f \nu} f_{-d e}{ }^{\lambda \rho} \gamma_{5} \gamma_{\nu} D_{\lambda} T_{b c f \rho}+$ H.c. & & 139 & $i \bar{T}^{a b c \mu} f_{s,+\mu}{ }^{\nu} h_{a}{ }^{d \lambda \rho} \gamma_{5} \gamma_{\nu} D_{\lambda} T_{b c d \rho}+$ H.c. & 205 & \\
\hline $\bar{T}^{a b c \mu} u_{a}{ }^{d \nu} u_{b}{ }^{e \lambda} f_{-d}{ }^{f}{ }_{\nu}{ }^{\rho} \gamma_{5} \gamma_{\rho} D_{\lambda} T_{c e f \mu}+$ H.c. & 53 & 140 & $i \bar{T}^{a b c \mu} f_{s,+\mu}{ }^{\nu} h_{a}{ }^{d \lambda \rho} \gamma_{5} \gamma_{\lambda} D_{\nu} T_{b c d \rho}+$ H.c. & 206 & \\
\hline $\bar{T}^{a b c \mu} u_{a}{ }^{d \nu} u_{b}{ }^{e \lambda} f_{-d}{ }^{f}{ }_{\lambda}{ }^{\rho} \gamma_{5} \gamma_{\nu} D_{\rho} T_{c e f \mu}+$ H.c. & 54 & 141 & $i \bar{T}^{a b c \mu} f_{s,+}{ }^{\nu \lambda} h_{a}{ }^{d}{ }_{\mu}^{\rho} \gamma_{5} \gamma_{\nu} D_{\lambda} T_{b c d \rho}$ & 207 & \\
\hline $\bar{T}^{a b c \mu} u_{a}{ }^{d}{ }_{\mu} u^{e f \nu} f_{-d e}{ }^{\lambda \rho} \gamma_{5} \gamma_{\lambda} D_{\nu} T_{b c f \rho}+$ H.c. & & 142 & $i \bar{T}^{a b c \mu} f_{s,+}{ }^{\nu \lambda} h_{a}{ }^{d}{ }_{\nu} \gamma_{5} \gamma_{\lambda} D_{\rho} T_{b c d \mu}$ & 208 & \\
\hline $\bar{T}^{a b c \mu} u_{a}{ }^{d \nu} u_{d}{ }^{e \lambda} f_{-b}{ }^{f}{ }_{\mu}^{\rho} \gamma_{5} \gamma_{\nu} D_{\lambda} T_{c e f \rho}+$ H.c. & 55 & 143 & $i \bar{T}^{a b c \mu} f_{s,+}{ }^{\nu \lambda} h_{a}{ }^{d}{ }_{\nu}{ }^{\rho} \gamma_{5} \gamma_{\rho} D_{\lambda} T_{b c d \mu}$ & 209 & \\
\hline $\bar{T}^{a b c \mu} u_{a}{ }^{d \nu} u_{d \mu}^{e} f_{-e}{ }^{f \lambda \rho} \gamma_{5} \gamma_{\nu} D_{\lambda} T_{b c f \rho}+$ H.c. & & 144 & $i \bar{T}^{a b c \mu} f_{s,+}{ }^{\nu \lambda} h_{a}{ }^{d \rho \sigma} \gamma_{5} \gamma_{\nu} D_{\lambda \rho \sigma} T_{b c d \mu}$ & 210 & \\
\hline $\bar{T}^{a b c \mu} u_{a}{ }^{d \nu} u_{b}{ }^{e \lambda} f_{-d}{ }^{f}{ }_{\lambda}{ }^{\rho} \gamma_{5} \gamma_{\rho} D_{\nu} T_{c e f \mu}+$ H.c. & 56 & 145 & $i \bar{T}^{a b c \mu} f_{+}{ }^{\operatorname{de\nu } \lambda} h_{e d}{ }^{\rho \sigma} \gamma_{5} \gamma_{\nu} D_{\lambda \rho \sigma} T_{a b c \mu}$ & & 418 \\
\hline $\bar{T}^{a b c \mu} u_{a}{ }^{d}{ }_{\mu} u^{e f \nu} f_{-f e}{ }^{\lambda \rho} \gamma_{5} \gamma_{\nu} D_{\lambda} T_{b c d \rho}+$ H.c. & & 146 & $i \bar{T}^{a b c \mu} \nabla^{\nu} f_{+a}{ }_{\nu}{ }_{\nu}^{\lambda} u_{b}{ }^{e \rho} \gamma_{5} \gamma_{\lambda} D_{\rho} T_{c d e \mu}$ & 211 & 419 \\
\hline $\bar{T}^{a b c \mu} u_{a}{ }^{d}{ }_{\mu} u^{e f \nu} f_{-f e}{ }^{\lambda \rho} \gamma_{5} \gamma_{\lambda} D_{\nu} T_{b c d \rho}+$ H.c. & & 147 & $i \bar{T}^{a b c \mu} \nabla^{\nu} f_{+a}{ }_{\nu}^{d}{ }^{\lambda} u_{b}{ }^{e \rho} \gamma_{5} \gamma_{\rho} D_{\lambda} T_{c d e \mu}$ & 212 & 420 \\
\hline $\bar{T}^{a b c \mu} u_{a}{ }^{d}{ }_{\mu} u^{e f \nu} f_{-f e}{ }^{\lambda \rho} \gamma_{5} \gamma_{\lambda} D_{\rho} T_{b c d \nu}+$ H.c. & & 148 & $i \bar{T}^{a b c \mu} \nabla^{\nu} f_{+a}{ }^{d \lambda \rho} u_{b}{ }^{e}{ }_{\nu} \gamma_{5} \gamma_{\lambda} D_{\rho} T_{c d e \mu}$ & 213 & 421 \\
\hline $\bar{T}^{a b c \mu} u_{a}{ }^{d \nu} u_{d}{ }^{e} f_{-e}{ }^{f \lambda \rho} \gamma_{5} \gamma_{\lambda} D_{\nu} T_{b c f \rho}+$ H.c. & & 149 & $i \bar{T}^{a b c \mu} \nabla^{\nu} f_{+a}{ }^{d}{ }_{\nu}{ }^{2} u_{d}{ }^{e \rho} \gamma_{5} \gamma_{\lambda} D_{\rho} T_{b c e \mu}+$ H.c. & 214 & 422 \\
\hline $\bar{T}^{a b c \mu} u_{a}{ }^{d \nu} u_{d}{ }_{\mu} f_{-e}{ }^{f \lambda \rho} \gamma_{5} \gamma_{\lambda} D_{\rho} T_{b c f \nu}+$ H.c. & & 150 & $i \bar{T}^{a b c \mu} \nabla^{\nu} f_{+a}{ }^{d}{ }_{\nu}{ }^{\lambda} u_{d}{ }^{e \rho} \gamma_{5} \gamma_{\rho} D_{\lambda} T_{b c e \mu}+$ H.c. & 215 & 423 \\
\hline $\bar{T}^{a b c \mu} u_{a}{ }^{d \nu} u_{d}{ }^{e \lambda} f_{-b}{ }_{\nu}{ }_{\nu}{ }^{\rho} \gamma_{5} \gamma_{\lambda} D_{\rho} T_{c e f \mu}+$ H.c. & 57 & 151 & $i \bar{T}^{a b c \mu} \nabla^{\nu} f_{+a}{ }^{d \lambda \rho} u_{d}{ }^{e}{ }_{\nu} \gamma_{5} \gamma_{\lambda} D_{\rho} T_{b c e \mu}+$ H.c. & 216 & 424 \\
\hline $\bar{T}^{a b c \mu} u_{a}{ }^{d \nu} u_{d}{ }^{e \lambda} f_{-b}{ }_{\nu}{ }_{\nu}{ } \gamma_{5} \gamma_{\rho} D_{\lambda} T_{c e f \mu}+$ H.c. & 58 & 152 & $i \bar{T}^{a b c \mu} \nabla^{\nu} f_{s,+\nu}^{\lambda} u_{a}^{d \rho} \gamma_{5} \gamma_{\lambda} D_{\rho} T_{b c d \mu}$ & 217 & \\
\hline $\bar{T}^{a b c \mu} u_{a}{ }^{d \nu} u^{e f}{ }_{\mu} f_{-d e}{ }^{\lambda \rho} \gamma_{5} \gamma_{\nu} D_{\lambda} T_{b c f \rho}+$ H.c. & & 153 & $i \bar{T}^{a b c \mu} \nabla^{\nu} f_{s,+\nu}{ }^{\lambda} u_{a}{ }^{d \rho} \gamma_{5} \gamma_{\rho} D_{\lambda} T_{b c d \mu}$ & 218 & \\
\hline $\bar{T}^{a b c \mu} u_{a}{ }^{d \nu} u_{d}{ }^{e \lambda} f_{-e^{f}{ }_{\mu}{ }^{\rho} \gamma_{5} \gamma_{\nu} D_{\lambda} T_{b c f \rho}+\text { H.c. }}$ & & 154 & $i \bar{T}^{a b c \mu} \nabla^{\nu} f_{s,+}{ }^{\lambda \rho} u_{a \nu}{ }^{d} \gamma_{5} \gamma_{\lambda} D_{\rho} T_{b c d \mu}$ & 219 & \\
\hline $\bar{T}^{a b c \mu} u_{a}{ }^{d \nu} u_{d}{ }^{e \lambda} f_{-e^{f}{ }_{\mu}^{\rho} \gamma_{5} \gamma_{\nu} D_{\rho} T_{b c f \lambda}+\text { H.c. }}$ & & 155 & $i \bar{T}^{a b c \mu} \nabla^{\nu} f_{+}{ }^{d e}{ }_{\nu}^{\lambda} u_{e d}{ }^{\rho} \gamma_{5} \gamma_{\lambda} D_{\rho} T_{a b c \mu}$ & & 425 \\
\hline $\bar{T}^{a b c \mu} u_{a}{ }^{d \nu} u_{d}{ }_{\nu}^{e} f_{-e}{ }^{f \lambda \rho} \gamma_{5} \gamma_{\lambda} D_{\rho} T_{b c f \mu}+$ H.c. & & 156 & $i \bar{T}^{a b c \mu} \nabla^{\nu} f_{+}{ }^{d e}{ }_{\nu}^{\lambda} u_{e d}{ }^{\rho} \gamma_{5} \gamma_{\rho} D_{\lambda} T_{a b c \mu}$ & & 426 \\
\hline $\bar{T}^{a b c \mu} u_{a}{ }^{d \nu} u_{d}^{e \lambda} f_{-e^{f}}{ }_{\mu}^{\rho} \gamma_{5} \gamma_{\lambda} D_{\nu} T_{b c f \rho}+$ H.c. & & 157 & $i \bar{T}^{a b c \mu} \nabla^{\nu} f_{+}{ }^{d e \lambda \rho} u_{e d \nu} \gamma_{5} \gamma_{\lambda} D_{\rho} T_{a b c \mu}$ & & 427 \\
\hline $\bar{T}^{a b c \mu} u_{a}^{d \nu} u_{d}^{e \lambda} f_{-e^{f}}{ }_{\mu}^{\rho} \gamma_{5} \gamma_{\lambda} D_{\rho} T_{b c f \nu}+$ H.c. & & 158 & $i \varepsilon^{\mu \nu \lambda \rho} \bar{T}^{a b c \sigma} f_{+a}{ }^{d}{ }_{\mu \sigma} f_{-b}{ }^{e}{ }_{\nu \lambda} T_{c d e \rho}+$ H.c. & 220 & 428 \\
\hline $\bar{T}^{a b c \mu} u_{a}{ }^{d \nu} u^{e f}{ }_{\mu} f_{-d e}{ }^{\lambda \rho} \gamma_{5} \gamma_{\lambda} D_{\nu} T_{b c f \rho}+$ H.c. & & 159 & $i \varepsilon^{\mu \nu \lambda \rho} \bar{T}^{a b c \sigma} f_{+a}{ }^{d}{ }_{\mu \sigma} f_{-d}{ }^{e}{ }_{\nu \lambda} T_{b c e \rho}+$ H.c. & 221 & 429 \\
\hline $\bar{T}^{a b c \mu} u_{a}{ }^{d \nu} u_{d}{ }^{e \lambda} f_{-e^{f}{ }_{\mu}{ }^{\rho} \gamma_{5} \gamma_{\rho} D_{\nu} T_{b c f \lambda}}+$ H.c. & & 160 & $i \varepsilon^{\mu \nu \lambda \rho} \bar{T}^{a b c \sigma} f_{+a}{ }^{d}{ }_{\mu \nu} f_{-d}{ }^{e} \lambda \sigma T_{b c e \rho}+$ H.c. & 222 & 430 \\
\hline $\bar{T}^{a b c \mu} u_{a}{ }^{d \nu} u_{d}{ }^{e \lambda} f_{-e^{f}{ }_{\mu}{ }^{\rho} \gamma_{5} \gamma_{\rho} D_{\lambda} T_{b c f \nu}}+$ H.c. & & 161 & $i \varepsilon^{\mu \nu \lambda \rho} \bar{T}_{\mu}^{a b c} f_{+a}{ }^{d \mu}{ }_{\nu} f_{-d}{ }^{e} \lambda \sigma T_{b c e \rho}+$ H.c. & 223 & 431 \\
\hline $\bar{T}^{a b c \mu} u_{a}{ }^{d \nu} u_{d}{ }^{e \lambda} f_{-e}{ }^{f}{ }_{\nu}^{\rho} \gamma_{5} \gamma_{\lambda} D_{\rho} T_{b c f \mu}+$ H.c. & & 162 & $i \varepsilon^{\mu \nu \lambda \rho} \bar{T}^{a b c \sigma} f_{s,+\mu \sigma} f_{-a}^{d}{ }^{d} T_{b c d \rho}+$ H.c. & 224 & \\
\hline $\bar{T}^{a b c \mu} u_{a}{ }^{d \nu} u^{e f}{ }_{\mu} f_{-f e}{ }^{\lambda \rho} \gamma_{5} \gamma_{\nu} D_{\lambda} T_{b c d \rho}+$ H.c. & & 163 & $i \varepsilon^{\mu \nu \lambda \rho} \bar{T}^{a b c \sigma} f_{+}{ }^{d e}{ }_{\mu \sigma} f_{-e d \nu \lambda} T_{a b c \rho}+$ H.c. & & 432 \\
\hline $\bar{T}^{a b c \mu} u_{a}{ }^{d \nu} u_{d}{ }^{e \lambda} f_{-e_{\nu}{ }^{\rho} \gamma_{5} \gamma_{\rho} D_{\lambda} T_{b c f \mu}+\text { H.c. }}$ & & 164 & $i \varepsilon^{\mu \nu \lambda \rho} \bar{T}^{a b c \sigma} f_{+a}{ }_{\mu \nu} h_{b}{ }^{e}{ }_{\lambda \sigma} T_{c d e \rho}+$ H.c. & 225 & 433 \\
\hline $\bar{T}^{a b c \mu} u_{a}{ }^{d \nu} u_{d}{ }^{e \lambda} f_{-e}{ }^{f} \lambda^{\rho} \gamma_{5} \gamma_{\nu} D_{\rho} T_{b c f \mu}+$ H.c. & & 165 & $i \varepsilon^{\mu \nu \lambda \rho} \bar{T}^{a b c \sigma} f_{+a}{ }^{d}{ }_{\mu \nu} h_{d}{ }^{e} \lambda \sigma T_{b c e \rho}+$ H.c. & 226 & 434 \\
\hline $\bar{T}^{a b c \mu} u_{a}{ }^{d \nu} u^{e f}{ }_{\mu} f_{-f e^{\lambda \rho} \gamma_{5} \gamma_{\lambda} D_{\nu} T_{b c d \rho}+\text { H.c. }}$ & & 166 & $i \varepsilon^{\mu \nu \lambda \rho} \bar{T}^{a b c}{ }_{\mu} f_{+a}{ }_{\nu}^{d}{ }^{\sigma} h_{d}{ }^{e} \lambda \sigma T_{b c e \rho}+$ H.c. & 227 & 435 \\
\hline $\bar{T}^{a b c \mu} u_{a}{ }^{d \nu} u_{d}{ }^{e \lambda} f_{-e}{ }^{f}{ }_{\lambda}^{\rho} \gamma_{5} \gamma_{\rho} D_{\nu} T_{b c f \mu}+$ H.c. & & 167 & $i \varepsilon^{\mu \nu \lambda \rho} \bar{T}^{a b c \sigma} f_{s,+\mu \nu} h_{a}^{d}{ }_{\lambda \sigma} T_{b c d \rho}+$ H.c. & 228 & \\
\hline $\bar{T}^{a b c \mu} u_{a}{ }^{d \nu} u^{e f \lambda} f_{-d e \mu}{ }^{\rho} \gamma_{5} \gamma_{\nu} D_{\lambda} T_{b c f \rho}+$ H.c. & & 168 & $i \varepsilon^{\mu \nu \lambda \rho} \bar{T}^{a b c \sigma} f_{+}{ }^{d e}{ }_{\mu \nu} h_{e d \lambda \sigma} T_{a b c \rho}+$ H.c. & & 436 \\
\hline $\bar{T}^{a b c \mu} u_{a}{ }^{d \nu} u^{e f \lambda} f_{-d e \nu}{ }^{\rho} \gamma_{5} \gamma_{\lambda} D_{\rho} T_{b c f \mu}+$ H.c. & & 169 & $i \varepsilon^{\mu \nu \lambda \rho} \bar{T}^{a b c}{ }_{\mu} \nabla^{\sigma} f_{+a}{ }^{d}{ }_{\nu \sigma} u_{b}{ }^{e}{ }_{\lambda} T_{c d e \rho}$ & 229 & 437 \\
\hline $\bar{T}^{a b c \mu} u_{a}{ }^{d \nu} u^{e f \lambda} f_{-d e \nu}{ }^{\rho} \gamma_{5} \gamma_{\rho} D_{\lambda} T_{b c f \mu}+$ H.c. & & 170 & $i \varepsilon^{\mu \nu \lambda \rho} \bar{T}^{a b c}{ }_{\mu} \nabla^{\sigma} f_{+a}{ }_{\nu \sigma}{ }_{\nu \sigma} u_{d}{ }^{e} T_{b c e \rho}+$ H.c. & 230 & 438 \\
\hline $\bar{T}^{a b c \mu} u_{a}^{d \nu} u^{e f \lambda} f_{-f e \mu}{ }^{\rho} \gamma_{5} \gamma_{\nu} D_{\lambda} T_{b c d \rho}$ & & 171 & $i \varepsilon^{\mu \nu \lambda \rho} \bar{T}^{a b c}{ }_{\mu} \nabla^{\sigma} f_{s,+\nu \sigma} u_{a}{ }^{d}{ }_{\lambda} T_{b c d \rho}$ & 231 & \\
\hline $\bar{T}^{a b c \mu} u_{a}^{d \nu} u^{e f \lambda} f_{-f e \mu}{ }^{\rho} \gamma_{5} \gamma_{\lambda} D_{\nu} T_{b c d \rho}$ & & 172 & $i \varepsilon^{\mu \nu \lambda \rho} \bar{T}^{a b c}{ }_{\mu} \nabla^{\sigma} f_{+}{ }_{d e}^{d e} u_{e d \lambda} T_{a b c \rho}$ & & 439 \\
\hline $\bar{T}^{a b c \mu} u^{d e}{ }_{\mu} u_{e d}{ }^{\nu} f_{-a}{ }^{f \lambda \rho} \gamma_{5} \gamma_{\nu} D_{\lambda} T_{b c f \rho}+$ H.c. & & 173 & $\bar{T}^{a b c \mu} f_{+a}{ }^{d}{ }_{\mu}^{\nu} f_{+b}{ }^{e}{ }_{\nu}^{\lambda} T_{c d e \lambda}$ & 232 & 440 \\
\hline
\end{tabular}


TABLE V. (Continued)

\begin{tabular}{|c|c|c|c|c|c|}
\hline$O_{n}^{\left(N_{f}, 4\right)}$ & $S U(2)$ & $S U(3)$ & $O_{n}^{\left(N_{f}, 4\right)}$ & $S U(2)$ & $S U(3)$ \\
\hline$\overline{\bar{T}^{a b c \mu} u^{d e}{ }_{\mu} u_{e d}{ }^{\nu} f_{-a}{ }^{f \lambda \rho} \gamma_{5} \gamma_{\lambda} D_{\nu} T_{b c f \rho}+\text { H.c. }}$ & & 174 & $\bar{T}^{a b c \mu} f_{+a}{ }^{d \nu \lambda} f_{+b}{ }^{e}{ }_{\nu \lambda} T_{c d e \mu}$ & 233 & 441 \\
\hline $\bar{T}^{a b c \mu} u^{d e}{ }_{\mu} u^{f}{ }_{d}^{\nu} f_{-e f}^{\lambda \rho} \gamma_{5} \gamma_{\nu} D_{\lambda} T_{a b c \rho}+$ H.c. & & 175 & $\bar{T}^{a b c \mu} f_{+a}{ }^{d \nu} f_{+d} e_{\nu}^{\lambda} T_{b c e \lambda}$ & 234 & 442 \\
\hline $\bar{T}^{a b c \mu} u^{d e}{ }_{\mu} u^{f}{ }_{d}^{\nu} f_{-e f}{ }^{\lambda \rho} \gamma_{5} \gamma_{\lambda} D_{\nu} T_{a b c \rho}+$ H.c. & & 176 & $\bar{T}^{a b c \mu} f_{+a} d \nu \lambda f_{+d}^{e}{ }_{\mu \nu} T_{b c e \lambda}$ & & 443 \\
\hline $\bar{T}^{a b c \mu} u^{d e \nu} u_{e d}{ }^{\lambda} f_{-a}^{f}{ }_{\mu}^{\rho} \gamma_{5} \gamma_{\nu} D_{\lambda} T_{b c f \rho}$ & & 177 & $\bar{T}^{a b c \mu} f_{+a} d \nu \lambda f_{+d}{ }^{e}{ }_{\nu \lambda} T_{b c e \mu}$ & & 444 \\
\hline $\bar{T}^{a b c \mu} u^{d e \nu} u_{e}{ }^{f \lambda} f_{-f d \nu}{ }^{\rho} \gamma_{5} \gamma_{\lambda} D_{\rho} T_{a b c \mu}+$ H.c. & & 178 & $\bar{T}^{a b c \mu} f_{+a}{ }^{d}{ }_{\mu}^{\nu} f_{+b}{ }^{e \lambda \rho} D_{\nu \lambda} T_{c d e \rho}$ & 235 & 445 \\
\hline $\bar{T}^{a b c \mu} u^{d e \nu} u_{e}^{f \lambda} f_{-f d \nu}^{\rho} \gamma_{5} \gamma_{\rho} D_{\lambda} T_{a b c \mu}+$ H.c. & & 179 & $\bar{T}^{a b c \mu} f_{+a}{ }^{d \nu \lambda} f_{+b}{ }^{e}{ }_{\nu}^{\rho} D_{\lambda \rho} T_{c d e \mu}$ & 236 & 446 \\
\hline $\bar{T}^{a b c \mu} u_{a}{ }^{d \nu} u_{b}{ }^{e \lambda} f_{-d}{ }^{f \rho \sigma} \gamma_{5} \gamma_{\rho} D_{\nu \lambda \sigma} T_{c e f \mu}+$ H.c. & 59 & 180 & $\bar{T}^{a b c \mu} f_{+a}{ }^{d}{ }_{\mu}^{\nu} f_{+d}{ }^{e \lambda \rho} D_{\nu \lambda} T_{b c e \rho}$ & 237 & 447 \\
\hline $\bar{T}^{a b c \mu} u_{a}{ }^{d \nu} u_{d}{ }^{e \lambda} f_{-e}{ }^{f \rho \sigma} \gamma_{5} \gamma_{\rho} D_{\nu \lambda \sigma} T_{b c f \mu}+$ H.c. & & 181 & $\bar{T}^{a b c \mu} f_{+a}{ }^{d \nu \lambda} f_{+d^{e}}^{e}{ }^{\rho} D_{\nu \rho} T_{b c e \lambda}$ & & 448 \\
\hline $\bar{T}^{a b c \mu} u_{a}{ }_{\mu}^{d} u_{b}{ }^{e \nu} h_{c}{ }^{f \lambda \rho} \gamma_{5} \gamma_{\nu} D_{\lambda} T_{d e f \rho}+$ H.c. & 60 & 182 & $\bar{T}^{a b c \mu} f_{+a} d \nu \lambda f_{+d^{e}}{ }_{\nu}^{\rho} D_{\lambda \rho} T_{b c e \mu}$ & & 449 \\
\hline $\bar{T}^{a b c \mu} u_{a}{ }^{d}{ }_{\mu} u_{b}{ }^{e \nu} h_{d}{ }^{f \lambda \rho} \gamma_{5} \gamma_{\nu} D_{\lambda} T_{c e f \rho}+$ H.c. & 61 & 183 & $i \bar{T}^{a b c \mu} f_{+a}{ }^{d}{ }_{\mu}^{\nu} f_{+b}{ }^{e \lambda \rho} \sigma_{\nu \lambda} T_{c d e \rho}$ & 238 & 450 \\
\hline $\bar{T}^{a b c \mu} u_{a}{ }^{d}{ }_{\mu} u_{b}{ }^{e \nu} h_{d}{ }^{f \lambda \rho} \gamma_{5} \gamma_{\lambda} D_{\nu} T_{c e f \rho}+$ H.c. & 62 & 184 & $i \bar{T}^{a b c \mu} f_{+a}{ }^{d \mu}{ }_{\mu}^{\nu} f_{+d}^{e \lambda \rho} \sigma_{\nu \lambda} T_{b c e \rho}$ & 239 & 451 \\
\hline $\bar{T}^{a b c \mu} u_{a}{ }^{d}{ }_{\mu} u_{b}{ }^{e \nu} h_{d}{ }^{f \lambda \rho} \gamma_{5} \gamma_{\lambda} D_{\rho} T_{c e f \nu}+$ H.c. & 63 & 185 & $\bar{T}^{a b c \mu} f_{+a \mu}{ }^{\mu \nu} f_{s,+\nu}{ }^{\lambda} T_{b c d \lambda}+$ H.c. & 240 & \\
\hline $\bar{T}^{a b c \mu} u_{a}{ }^{d}{ }_{\mu} u_{b}{ }^{e \nu} h_{e}{ }^{f \lambda \rho} \gamma_{5} \gamma_{\nu} D_{\lambda} T_{c d f \rho}+$ H.c. & 64 & 186 & $\bar{T}^{a b c \mu} f_{+a}^{d \nu \lambda} f_{s,+\nu \lambda} T_{b c d \mu}$ & 241 & \\
\hline $\bar{T}^{a b c \mu} u_{a}{ }^{d}{ }_{\mu} u_{b}{ }^{e \nu} h_{e}{ }^{f \lambda \rho} \gamma_{5} \gamma_{\lambda} D_{\nu} T_{c d f \rho}+$ H.c. & 65 & 187 & $\bar{T}^{a b c \mu} f_{+a}{ }^{d}{ }_{\mu}^{\nu} f_{s,+}{ }^{\lambda \rho} D_{\nu \lambda} T_{b c d \rho}+$ H.c. & 242 & \\
\hline $\bar{T}^{a b c \mu} u_{a}{ }_{\mu}^{d} u_{b}{ }^{e \nu} h_{e}{ }^{f \lambda \rho} \gamma_{5} \gamma_{\lambda} D_{\rho} T_{c d f \nu}+$ H.c. & 66 & 188 & $\bar{T}^{a b c \mu} f_{+a}{ }^{d \nu \lambda} f_{s,+\nu}^{\rho} D_{\lambda \rho} T_{b c d \mu}$ & 243 & \\
\hline $\bar{T}^{a b c \mu} u_{a}{ }_{\mu}^{d} u_{d}{ }^{e \nu} h_{b}{ }^{f \lambda \rho} \gamma_{5} \gamma_{\lambda} D_{\nu} T_{c e f \rho}+$ H.c. & 67 & 189 & $i \bar{T}^{a b c \mu} f_{+a}{ }^{d}{ }_{\mu}^{\nu} f_{s,+}{ }^{\lambda \rho} \sigma_{\nu \lambda} T_{b c d \rho}+$ H.c. & 244 & \\
\hline $\bar{T}^{a b c \mu} u_{a}{ }_{\mu}^{d} u_{d}{ }^{e \nu} h_{e}{ }^{f \lambda \rho} \gamma_{5} \gamma_{\nu} D_{\lambda} T_{b c f \rho}+$ H.c. & & 190 & $\bar{T}^{a b c \mu} f_{s,+\mu}^{\nu} f_{s,+\nu}^{\lambda} T_{a b c \lambda}$ & 245 & \\
\hline $\bar{T}^{a b c \mu} u_{a}{ }^{d}{ }_{\mu} u_{d}{ }^{e \nu} h_{e}{ }^{f \lambda \rho} \gamma_{5} \gamma_{\lambda} D_{\nu} T_{b c f \rho}+$ H.c. & & 191 & $\bar{T}^{a b c \mu} f_{s,+}{ }^{\nu \lambda} f_{s,+\nu \lambda} T_{a b c \mu}$ & 246 & \\
\hline $\bar{T}^{a b c \mu} u_{a}{ }^{d}{ }_{\mu} u_{d}{ }^{e \nu} h_{e}{ }^{f \lambda \rho} \gamma_{5} \gamma_{\lambda} D_{\rho} T_{b c f \nu}+$ H.c. & & 192 & $\bar{T}^{a b c \mu} f_{s,+\mu}^{\nu} f_{s,+}^{\lambda \rho} D_{\nu \lambda} T_{a b c \rho}$ & 247 & \\
\hline $\bar{T}^{a b c \mu} u_{a}{ }^{d \nu} u_{b}{ }^{e \lambda} h_{d}^{f}{ }_{\mu}^{\rho} \gamma_{5} \gamma_{\nu} D_{\rho} T_{c e f \lambda}+$ H.c. & 68 & 193 & $\bar{T}^{a b c \mu} f_{s,+}{ }^{\nu \lambda} f_{s,+\nu}{ }^{\rho} D_{\lambda \rho} T_{a b c \mu}$ & 248 & \\
\hline $\bar{T}^{a b c \mu} u_{a}{ }^{d \nu} u_{b}{ }^{e}{ }_{\nu} h_{d}{ }^{f \lambda \rho} \gamma_{5} \gamma_{\lambda} D_{\rho} T_{c e f \mu}+$ H.c. & 69 & 194 & $i \bar{T}^{a b c \mu} f_{s,+\mu}{ }^{\nu} f_{s,+}^{\lambda \rho} \sigma_{\nu \lambda} T_{a b c \rho}$ & 249 & \\
\hline $\bar{T}^{a b c \mu} u_{a}{ }^{d \nu} u_{b}{ }^{e \lambda} h_{d}{ }_{\mu}^{f}{ }^{\rho} \gamma_{5} \gamma_{\lambda} D_{\rho} T_{c e f \nu}+$ H.c. & 70 & 195 & $i \bar{T}^{a b c \mu} f_{+}{ }^{d e}{ }_{\mu}^{\nu} f_{+e d}{ }^{\lambda \rho} \sigma_{\nu \lambda} T_{a b c \rho}$ & & 452 \\
\hline $\bar{T}^{a b c \mu} u_{a}{ }^{d \nu} u_{b}{ }^{e \lambda} h_{d}{ }_{\nu}^{f}{ }_{\nu}^{\rho} \gamma_{5} \gamma_{\lambda} D_{\rho} T_{c e f \mu}+$ H.c. & 71 & 196 & $\bar{T}^{a b c \mu} u_{a}{ }^{d}{ }_{\mu} u_{b}{ }^{e \nu} \chi_{+c}{ }^{f} T_{d e f \nu}$ & 250 & 453 \\
\hline $\bar{T}^{a b c \mu} u_{a}{ }^{d}{ }_{\mu} u^{e f \nu} h_{d e}{ }^{\lambda \rho} \gamma_{5} \gamma_{\nu} D_{\lambda} T_{b c f \rho}+$ H.c. & & 197 & $\bar{T}^{a b c \mu} u_{a}{ }^{d \nu} u_{b}{ }^{e}{ }_{\nu} \chi_{+c}{ }^{f} T_{d e f \mu}$ & 251 & 454 \\
\hline $\bar{T}^{a b c \mu} u_{a}{ }^{d \nu} u_{b}{ }^{e \lambda} h_{d}^{f}{ }_{\nu}^{\rho} \gamma_{5} \gamma_{\rho} D_{\lambda} T_{c e f \mu}+$ H.c. & 72 & 198 & $\bar{T}^{a b c \mu} u_{a}{ }^{d}{ }_{\mu} u_{b}{ }^{e \nu} \chi_{+d}{ }^{f} T_{c e f \nu}+$ H.c. & 252 & 455 \\
\hline $\bar{T}^{a b c \mu} u_{a}{ }^{d \nu} u_{b}{ }^{e \lambda} h_{d}{ }^{f}{ }_{\lambda}^{\rho} \gamma_{5} \gamma_{\nu} D_{\rho} T_{c e f \mu}+$ H.c. & 73 & 199 & $\bar{T}^{a b c \mu} u_{a}{ }^{d}{ }_{\mu} u_{b}{ }^{e \nu} \chi_{+e}{ }^{f} T_{c d f \nu}+$ H.c. & 253 & 456 \\
\hline $\bar{T}^{a b c \mu} u_{a}{ }^{d}{ }_{\mu} u^{e f \nu} h_{d e}{ }^{\lambda \rho} \gamma_{5} \gamma_{\lambda} D_{\nu} T_{b c f \rho}+$ H.c. & & 200 & $\bar{T}^{a b c \mu} u_{a}{ }^{d \nu} u_{b}{ }^{e}{ }_{\nu} \chi_{+d}{ }^{f} T_{c e f \mu}+$ H.c. & 254 & 457 \\
\hline $\bar{T}^{a b c \mu} u_{a}{ }^{d \nu} u_{b}{ }^{e \lambda} h_{d}{ }_{\lambda}^{f}{ }_{\lambda}^{\rho} \gamma_{5} \gamma_{\rho} D_{\nu} T_{c e f \mu}+$ H.c. & 74 & 201 & $\bar{T}^{a b c \mu} u_{a}{ }^{d}{ }_{\mu} u_{d}^{e \nu} \chi_{+b}{ }^{f} T_{c e f \nu}$ & 255 & 458 \\
\hline $\bar{T}^{a b c \mu} u_{a}{ }^{d}{ }_{\mu} u^{e f \nu} h_{f e}{ }^{\lambda \rho} \gamma_{5} \gamma_{\nu} D_{\lambda} T_{b c d \rho}+$ H.c. & & 202 & $\bar{T}^{a b c \mu} u_{a}{ }^{d \nu} u_{d}{ }^{e}{ }_{\mu} \chi_{+b}{ }^{f} T_{c e f \nu}$ & 256 & 459 \\
\hline $\bar{T}^{a b c \mu} u_{a}{ }^{d}{ }_{\mu} u^{e f \nu} h_{f e}{ }^{\lambda \rho} \gamma_{5} \gamma_{\lambda} D_{\rho} T_{b c d \nu}+$ H.c. & & 203 & $\bar{T}^{a b c \mu} u_{a}{ }^{d \nu} u_{d}{ }^{e}{ }_{\nu} \chi_{+b}{ }^{f} T_{c e f \mu}$ & 257 & 460 \\
\hline $\bar{T}^{a b c \mu} u_{a}{ }^{d \nu} u_{d}{ }^{e} h_{e}{ }^{f \lambda \rho} \gamma_{5} \gamma_{\lambda} D_{\nu} T_{b c f \rho}+$ H.c. & & 204 & $\bar{T}^{a b c \mu} u_{a}{ }^{d}{ }_{\mu} u_{d}{ }^{e \nu} \chi_{+e}{ }^{f} T_{b c f \nu}+$ H.c. & & 461 \\
\hline $\bar{T}^{a b c \mu} u_{a}{ }^{d \nu} u_{d}{ }^{e}{ }_{\mu} h_{e}{ }^{f \lambda \rho} \gamma_{5} \gamma_{\lambda} D_{\rho} T_{b c f \nu}+$ H.c. & & 205 & $\bar{T}^{a b c \mu} u_{a}{ }^{d \nu} u_{d}{ }^{e} \chi_{+e}{ }^{f} T_{b c f \nu}+$ H.c. & & 462 \\
\hline $\bar{T}^{a b c \mu} u_{a}{ }^{d \nu} u_{d}{ }^{e \lambda} h_{b}{ }_{\nu}^{f}{ }_{\nu} \gamma_{5} \gamma_{\rho} D_{\lambda} T_{c e f \mu}+$ H.c. & 75 & 206 & $\bar{T}^{a b c \mu} u_{a}{ }^{d \nu} u_{d}{ }^{e} \chi_{\nu+e}{ }^{f} T_{b c f \mu}+$ H.c. & & 463 \\
\hline $\bar{T}^{a b c \mu} u_{a}{ }^{d \nu} u^{e f}{ }_{\mu} h_{d e}{ }^{\lambda \rho} \gamma_{5} \gamma_{\nu} D_{\lambda} T_{b c f \rho}+$ H.c. & & 207 & $\bar{T}^{a b c \mu} u_{a \mu}{ }_{\mu} u^{e f \nu} \chi_{+d e} T_{b c f \nu}$ & & 464 \\
\hline $\bar{T}^{a b c \mu} u_{a}{ }^{d \nu} u_{d}{ }_{\nu}^{e} h_{e}{ }^{f \lambda \rho} \gamma_{5} \gamma_{\lambda} D_{\rho} T_{b c f \mu}+$ H.c. & & 208 & $\bar{T}^{a b c \mu} u_{a}^{d \nu} u^{e f}{ }_{\mu} \chi_{+d e} T_{b c f \nu}$ & & 465 \\
\hline $\bar{T}^{a b c \mu} u_{a}{ }^{d \nu} u_{d}{ }^{e \lambda} h_{e}^{f}{ }_{\mu}^{\rho} \gamma_{5} \gamma_{\lambda} D_{\rho} T_{b c f \nu}+$ H.c. & & 209 & $\bar{T}^{a b c \mu} u_{a}{ }^{d \nu} u^{e f}{ }_{\nu} \chi_{+d e} T_{b c f \mu}$ & & 466 \\
\hline $\bar{T}^{a b c \mu} u_{a}{ }^{d \nu} u_{d}{ }^{e \lambda} h_{e}^{f}{ }_{\nu}^{\rho} \gamma_{5} \gamma_{\lambda} D_{\rho} T_{b c f \mu}+$ H.c. & & 210 & $\bar{T}^{a b c \mu} u_{a}{ }^{d}{ }_{\mu} u^{e f \nu} \chi_{+f e} T_{b c d \nu}+$ H.c. & & 467 \\
\hline $\bar{T}^{a b c \mu} u_{a}{ }^{d \nu} u^{e f}{ }_{\mu} h_{f e}{ }^{\lambda \rho} \gamma_{5} \gamma_{\nu} D_{\lambda} T_{b c d \rho}+$ H.c. & & 211 & $\bar{T}^{a b c \mu} u_{a}{ }^{d \nu} u^{e f}{ }_{\nu} \chi_{+f e} T_{b c d \mu}$ & & 468 \\
\hline $\bar{T}^{a b c \mu} u_{a}{ }^{d \nu} u_{d}{ }^{e \lambda} h_{e}^{f}{ }_{\nu}^{\rho} \gamma_{5} \gamma_{\rho} D_{\lambda} T_{b c f \mu}+$ H.c. & & 212 & $\bar{T}^{a b c \mu} u^{d e}{ }_{\mu} u_{e d}{ }^{\nu} \chi_{+a}{ }^{f} T_{b c f \nu}$ & & 469 \\
\hline $\bar{T}^{a b c \mu} u_{a}{ }^{d \nu} u_{d}{ }^{e \lambda} h_{e}{ }^{f}{ }_{\lambda}^{\rho} \gamma_{5} \gamma_{\nu} D_{\rho} T_{b c f \mu}+$ H.c. & & 213 & $\bar{T}^{a b c \mu} u^{d e \nu} u_{e d \nu} \chi_{+a}^{f} T_{b c f \mu}$ & & 470 \\
\hline $\bar{T}^{a b c \mu} u_{a}{ }^{d \nu} u_{d}{ }^{e \lambda} h_{e}{ }^{f}{ }_{\lambda}^{\rho} \gamma_{5} \gamma_{\rho} D_{\nu} T_{b c f \mu}+$ H.c. & & 214 & $\bar{T}^{a b c \mu} u^{d e}{ }_{\mu} u_{e}{ }^{f \nu} \chi_{+f d} T_{a b c \nu}$ & & 471 \\
\hline $\bar{T}^{a b c \mu} u_{a}^{d \nu} u^{e f \lambda} h_{d e \nu}{ }^{\rho} \gamma_{5} \gamma_{\rho} D_{\lambda} T_{b c f \mu}+$ H.c. & & 215 & $\bar{T}^{a b c \mu} u_{a}^{d \nu} u_{b}^{e \lambda} \chi_{+c}^{f} D_{\nu \lambda} T_{d e f \mu}$ & 258 & 472 \\
\hline $\bar{T}^{a b c \mu} u^{d e}{ }_{\mu} u_{d}^{f}{ }^{\nu} h_{e f}{ }^{\lambda \rho} \gamma_{5} \gamma_{\nu} D_{\lambda} T_{a b c \rho}+$ H.c. & & 216 & $\bar{T}^{a b c \mu} u_{a}^{d \nu} u_{b}^{e \lambda} \chi_{+d}^{f} D_{\nu \lambda} T_{c e f \mu}+$ H.c. & 259 & 473 \\
\hline $\bar{T}^{a b c \mu} u^{d e}{ }_{\mu} u^{f}{ }_{d}^{\nu} h_{e f}{ }^{\lambda \rho} \gamma_{5} \gamma_{\lambda} D_{\nu} T_{a b c \rho}+$ H.c. & & 217 & $\bar{T}^{a b c \mu} u_{a}{ }^{d \nu} u_{d}{ }^{e \lambda} \chi_{+b}{ }^{f} D_{\nu \lambda} T_{c e f \mu}$ & 260 & 474 \\
\hline $\bar{T}^{a b c \mu} u^{d e \nu} u_{e}^{f \lambda} h_{f d \nu}{ }^{\rho} \gamma_{5} \gamma_{\lambda} D_{\rho} T_{a b c \mu}+$ H.c. & & 218 & $\bar{T}^{a b c \mu} u_{a}^{d \nu} u_{d}^{e \lambda} \chi_{+e}{ }^{f} D_{\nu \lambda} T_{b c f \mu}+$ H.c. & & 475 \\
\hline $\bar{T}^{a b c \mu} u^{d e \nu} u_{e}^{f \lambda} h_{f d \nu}{ }^{\rho} \gamma_{5} \gamma_{\rho} D_{\lambda} T_{a b c \mu}+$ H.c. & & 219 & $\bar{T}^{a b c \mu} u_{a}{ }^{d \nu} u^{e f \lambda} \chi_{+d e} D_{\nu \lambda} T_{b c f \mu}$ & & 476 \\
\hline $\bar{T}^{a b c \mu} u_{a}{ }^{d \nu} u_{b}{ }^{e \lambda} h_{d}{ }^{f \rho \sigma} \gamma_{5} \gamma_{\nu} D_{\lambda \rho \sigma} T_{c e f \mu}+$ H.c. & 76 & 220 & $\bar{T}^{a b c \mu} u_{a}{ }^{d \nu} u^{e f \lambda} \chi_{+f e} D_{\nu \lambda} T_{b c d \mu}$ & & 477 \\
\hline $\bar{T}^{a b c \mu} u_{a}{ }^{d \nu} u_{b}{ }^{e \lambda} h_{d}{ }^{f \rho \sigma} \gamma_{5} \gamma_{\lambda} D_{\nu \rho \sigma} T_{c e f \mu}+$ H.c. & 77 & 221 & $\bar{T}^{a b c \mu} u^{d e \nu} u_{e d}^{\lambda} \chi_{+a}{ }^{f} D_{\nu \lambda} T_{b c f \mu}$ & & 478 \\
\hline
\end{tabular}


TABLE V. (Continued)

\begin{tabular}{|c|c|c|c|c|c|}
\hline$O_{n}^{\left(N_{f}, 4\right)}$ & $S U(2)$ & $S U(3)$ & $O_{n}^{\left(N_{f}, 4\right)}$ & $S U(2)$ & $S U(3)$ \\
\hline $\bar{T}^{a b c \mu} u_{a}{ }^{d \nu} u_{b}{ }^{e \lambda} h_{d}{ }^{f \rho \sigma} \gamma_{5} \gamma_{\rho} D_{\nu \lambda \sigma} T_{c e f \mu}+$ H.c. & 78 & 222 & $\bar{T}^{a b c \mu} u_{a}{ }^{d}{ }_{\mu} u_{b}{ }^{e \nu} \chi_{+, s} T_{c d e \nu}$ & 261 & 479 \\
\hline $\bar{T}^{a b c \mu} u_{a}^{d \nu} u_{d}^{e \lambda} h_{e}^{f \rho \sigma} \gamma_{5} \gamma_{\nu} D_{\lambda \rho \sigma} T_{b c f \mu}+$ H.c. & & 223 & $\bar{T}^{a b c \mu} u_{a}{ }^{d \nu} u_{b}{ }^{e}{ }_{\nu} \chi_{+, s} T_{c d e \mu}$ & 262 & 480 \\
\hline $\bar{T}^{a b c \mu} u_{a}{ }^{d \nu} u_{d}{ }^{e \lambda} h_{e}{ }^{f \rho \sigma} \gamma_{5} \gamma_{\lambda} D_{\nu \rho \sigma} T_{b c f \mu}+$ H.c. & & 224 & $\bar{T}^{a b c \mu} u_{a}{ }^{d}{ }_{\mu} u_{d}{ }^{e \nu} \chi_{+, s} T_{b c e \nu}$ & 263 & 481 \\
\hline $\bar{T}^{a b c \mu} u_{a}{ }^{d \nu} u_{d}^{e \lambda} h_{e}^{f \rho \sigma} \gamma_{5} \gamma_{\rho} D_{\nu \lambda \sigma} T_{b c f \mu}+$ H.c. & & 225 & $\bar{T}^{a b c \mu} u_{a}{ }^{d \nu} u_{d}{ }^{e}{ }_{\mu} \chi_{+, s} T_{b c e \nu}$ & 264 & 482 \\
\hline $\bar{T}^{a b c \mu} u^{d e \nu} u_{e}^{f \lambda} h_{f d} \rho \sigma \gamma_{5} \gamma_{\nu} D_{\lambda \rho \sigma} T_{a b c \mu}+$ H.c. & & 226 & $\bar{T}^{a b c \mu} u_{a}{ }^{d \nu} u_{d}{ }^{e}{ }_{\nu} \chi_{+, s} T_{b c e \mu}$ & 265 & 483 \\
\hline$\varepsilon^{\mu \nu \lambda \rho} \bar{T}^{a b c}{ }_{\mu} u_{a}{ }^{d}{ }_{\nu} u_{b}{ }_{\lambda}{ }_{\lambda} f_{-d}{ }_{\rho}^{f}{ }^{\sigma} T_{c e f \sigma}+$ H.c. & 79 & 227 & $\bar{T}^{a b c \mu} u^{d e}{ }_{\mu} u_{e d}{ }^{\nu} \chi_{+, s} T_{a b c \nu}$ & & 484 \\
\hline$\varepsilon^{\mu \nu \lambda \rho} \bar{T}^{a b c}{ }_{\mu} u_{a}{ }^{d}{ }_{\nu} u_{d}{ }^{e} f_{-b}{ }^{f}{ }_{\rho}^{\sigma} T_{c e f \sigma}+$ H.c. & 80 & 228 & $\bar{T}^{a b c \mu} u^{d e \nu} u_{e d \nu} \chi_{+, s} T_{a b c \mu}$ & & 485 \\
\hline$\varepsilon^{\mu \nu \lambda \rho} \bar{T}^{a b c}{ }_{\mu} u_{a}{ }^{d}{ }_{\nu} u_{d}{ }^{e} f_{-e^{f}{ }_{\rho}{ }^{\sigma}} T_{b c f \sigma}+$ H.c. & & 229 & $\bar{T}^{a b c \mu} u_{a}{ }^{d \nu} u_{b}{ }^{e \lambda} \chi_{+, s} D_{\nu \lambda} T_{c d e \mu}$ & 266 & 486 \\
\hline$\varepsilon^{\mu \nu \lambda \rho} \bar{T}^{a b c}{ }_{\mu} u_{a}{ }^{d}{ }_{\nu} u^{e f}{ }_{\lambda} f_{-b e \rho}{ }^{\sigma} T_{c d f \sigma}+$ H.c. & 81 & 230 & $\bar{T}^{a b c \mu} u_{a}{ }^{d \nu} u_{d}{ }^{e \lambda} \chi_{+, s} D_{\nu \lambda} T_{b c e \mu}$ & 267 & 487 \\
\hline$\varepsilon^{\mu \nu \lambda \rho} \bar{T}^{a b c}{ }_{\mu} u_{a}{ }^{d}{ }_{\nu} u_{b}{ }^{e \sigma} f_{-c}{ }^{f}{ }_{\lambda \rho} T_{d e f \sigma}+$ H.c. & 82 & 231 & $\bar{T}^{a b c \mu} u^{d e \nu} u_{e d}^{\lambda} \chi_{+, s} D_{\nu \lambda} T_{a b c \mu}$ & & 488 \\
\hline$\varepsilon^{\mu \nu \lambda \rho} \bar{T}^{a b c}{ }_{\mu} u_{a}{ }^{d}{ }_{\nu} u^{e f}{ }_{\lambda} f_{-d e \rho}{ }^{\sigma} T_{b c f \sigma}+$ H.c. & & 232 & $\bar{T}^{a b c \mu} f_{-a}{ }^{d \nu \lambda} \chi_{+d}{ }^{e} \gamma_{5} \gamma_{\nu} D_{\lambda} T_{b c e \mu}+$ H.c. & 268 & 489 \\
\hline$\varepsilon^{\mu \nu \lambda \rho} \bar{T}^{a b c}{ }_{\mu} u_{a}{ }^{d}{ }_{\nu} u_{b}{ }^{e \sigma} f_{-d}{ }^{f}{ }_{\lambda \rho} T_{c e f \sigma}+$ H.c. & 83 & 233 & $\bar{T}^{a b c \mu} h_{a}{ }^{d \nu \lambda} \chi_{+d}{ }^{e} \gamma_{5} \gamma_{\nu} D_{\lambda} T_{b c e \mu}+$ H.c. & 269 & 490 \\
\hline$\varepsilon^{\mu \nu \lambda \rho} \bar{T}^{a b c}{ }_{\mu} u_{a}{ }^{d}{ }_{\nu} u_{b}{ }^{e \sigma} f_{-e}{ }^{f}{ }_{\lambda \rho} T_{c d f \sigma}+$ H.c. & 84 & 234 & $\bar{T}^{a b c \mu} u_{a}{ }^{d \nu} \nabla^{\lambda} \chi_{+d}^{e} \gamma_{5} \gamma_{\lambda} D_{\nu} T_{b c e \mu}+$ H.c. & 270 & 491 \\
\hline$\varepsilon^{\mu \nu \lambda \rho} \bar{T}^{a b c}{ }_{\mu} u_{a}{ }^{d}{ }_{\nu} u^{e f}{ }_{\lambda} f_{-f e \rho}{ }^{\sigma} T_{b c d \sigma}+$ H.c. & & 235 & $\varepsilon^{\mu \nu \lambda \rho} \bar{T}^{a b c}{ }_{\mu} f_{-a}{ }^{d}{ }_{\nu \lambda} \chi_{+d}{ }^{e} T_{b c e \rho}+$ H.c. & 271 & 492 \\
\hline$\varepsilon^{\mu \nu \lambda \rho} \bar{T}^{a b c}{ }_{\mu} u_{a}{ }^{d}{ }_{\nu} u_{b}{ }^{e \sigma} f_{-d}{ }^{f} \lambda \sigma T_{c e f \rho}+$ H.c. & 85 & 236 & $\varepsilon^{\mu \nu \lambda \rho} \bar{T}^{a b c}{ }_{\mu} u_{a}{ }^{d}{ }_{\nu} \nabla_{\lambda{ }_{+d}{ }^{e}} T_{b c e \rho}+$ H.c. & 272 & 493 \\
\hline$\varepsilon^{\mu \nu \lambda \rho} \bar{T}^{a b c}{ }_{\mu} u_{a}{ }^{d}{ }_{\nu} u_{d}{ }^{e \sigma} f_{-b}{ }_{\lambda \rho} T_{c e f \sigma}+$ H.c. & 86 & 237 & $\bar{T}^{a b c \mu} \nabla^{\nu} \nabla_{\nu} \chi_{+a}{ }^{d} T_{b c d \mu}$ & 273 & 494 \\
\hline$\varepsilon^{\mu \nu \lambda \rho} \bar{T}^{a b c}{ }_{\mu} u_{a}{ }^{d}{ }_{\nu} u_{d}{ }^{e \sigma} f_{-e}{ }^{f}{ }_{\lambda \rho} T_{b c f \sigma}+$ H.c. & & 238 & $\bar{T}^{a b c \mu} \nabla^{\nu} \nabla_{\nu} \chi_{+, s} T_{a b c \mu}$ & 274 & 495 \\
\hline$\varepsilon^{\mu \nu \lambda \rho} \bar{T}^{a b c}{ }_{\mu} u_{a}{ }^{d}{ }_{\nu} u_{d}{ }^{e \sigma} f_{-e}{ }^{f} \lambda \sigma T_{b c f \rho}+$ H.c. & & 239 & $i \bar{T}^{a b c \mu} f_{+a}{ }^{d}{ }_{\mu}^{\nu} \chi_{+b}{ }^{e} T_{c d e \nu}$ & 275 & 496 \\
\hline$\varepsilon^{\mu \nu \lambda \rho} \bar{T}_{\mu}^{a b c} u_{a}{ }^{d}{ }_{\nu} u^{e f \sigma} f_{-b e \lambda \rho} T_{c d f \sigma}+$ H.c. & 87 & 240 & $i \bar{T}^{a b c \mu} f_{+a}{ }^{d}{ }_{\mu}^{\nu} \chi_{+d}{ }^{e} T_{b c e \nu}+$ H.c. & 276 & 497 \\
\hline$\varepsilon^{\mu \nu \lambda \rho} \bar{T}_{\mu}^{a b c} u_{a}{ }^{d}{ }_{\nu} u^{e f \sigma} f_{-d e \lambda \rho} T_{b c f \sigma}+$ H.c. & & 241 & $i \bar{T}^{a b c \mu} f_{s,+\mu}{ }^{\nu} \chi_{+a}{ }^{d} T_{b c d \nu}$ & 277 & \\
\hline$\varepsilon^{\mu \nu \lambda \rho} \bar{T}_{\mu}^{a b c}{ }_{\mu} u_{a}{ }^{d}{ }_{\nu} u^{e f \sigma} f_{-f e \lambda \rho} T_{b c d \sigma}+$ H.c. & & 242 & $i \bar{T}^{a b c \mu} f_{+}{ }^{d e}{ }_{\mu}{ }^{\nu} \chi_{+e d} T_{a b c \nu}$ & & 498 \\
\hline$\varepsilon^{\mu \nu \lambda \rho} \bar{T}_{\mu}^{a b c}{ }_{\mu} u_{\nu}^{d e} u_{e}{ }_{\lambda} f_{-a d \rho}{ }^{\sigma} T_{b c f \sigma}+$ H.c. & & 243 & $i \bar{T}^{a b c \mu} f_{+a}{ }^{d}{ }_{\mu}^{\nu} \chi_{+, s} T_{b c d \nu}$ & 278 & 499 \\
\hline$\varepsilon^{\mu \nu \lambda \rho} \bar{T}_{\mu}^{a b c}{ }_{\mu} u^{d e}{ }_{\nu} u_{e}{ }_{\lambda} f_{-f d \rho}{ }^{\sigma} T_{a b c \sigma}+$ H.c. & & 244 & $i \bar{T}^{a b c \mu} f_{s,+\mu}{ }^{\nu} \chi_{+, s} T_{a b c \nu}$ & 279 & \\
\hline$\varepsilon^{\mu \nu \lambda \rho} \bar{T}^{a b c}{ }_{\mu} u_{a}{ }^{d \sigma} u_{d}{ }^{e} f_{-e} f_{\lambda \rho} T_{b c f \sigma}+$ H.c. & & 245 & $\bar{T}^{a b c \mu} \chi_{+a}{ }^{d} \chi_{+b}{ }^{e} T_{c d e \mu}$ & 280 & 500 \\
\hline$\varepsilon^{\mu \nu \lambda \rho} \bar{T}^{a b c}{ }_{\mu} u^{d e}{ }_{\nu} u_{e d}{ }^{\sigma} f_{-a}{ }_{\lambda \rho}^{f} T_{b c f \sigma}+$ H.c. & & 246 & $\bar{T}^{a b c \mu} \chi_{+a}{ }^{d} \chi_{+d}^{e} T_{b c e \mu}$ & 281 & 501 \\
\hline$\varepsilon^{\mu \nu \lambda \rho} \bar{T}^{a b c}{ }_{\mu} u^{d e}{ }_{\nu} u_{e}{ }^{f \sigma} f_{-a d \lambda \rho} T_{b c f \sigma}+$ H.c. & & 247 & $\bar{T}^{a b c \mu} \chi_{+}{ }^{d e} \chi_{+e d} T_{a b c \mu}$ & & 502 \\
\hline$\varepsilon^{\mu \nu \lambda \rho} \bar{T}^{a b c}{ }_{\mu} u_{a}{ }^{d \sigma} u^{e f}{ }_{\nu} f_{-f e \lambda \rho} T_{b c d \sigma}+$ H.c. & & 248 & $\bar{T}^{a b c \mu} \chi_{+a}{ }^{d} \chi_{+, s} T_{b c d \mu}$ & 282 & 503 \\
\hline$\varepsilon^{\mu \nu \lambda \rho} \bar{T}^{a b c}{ }_{\mu} u_{a}{ }^{d}{ }_{\nu} u_{b}{ }_{\lambda}{ }_{\lambda} h_{d}{ }_{\rho}^{f}{ }_{\rho}^{\sigma} T_{c e f \sigma}+$ H.c. & 88 & 249 & $\bar{T}^{a b c \mu} \chi_{+, s} \chi_{+, s} T_{a b c \mu}$ & 283 & 504 \\
\hline$\varepsilon^{\mu \nu \lambda \rho} \bar{T}^{a b c}{ }_{\mu} u_{a}{ }^{d}{ }_{\nu} u_{d}{ }_{\lambda}{ }_{\lambda} h_{b}{ }^{f}{ }_{\rho}{ }^{\sigma} T_{c e f \sigma}+$ H.c. & 89 & 250 & $i \bar{T}^{a b c \mu} u_{a}^{d \nu} u_{b}{ }^{e \lambda} \chi_{-d}{ }^{f} \gamma_{5} \gamma_{\nu} D_{\lambda} T_{c e f \mu}+$ H.c. & 284 & 505 \\
\hline$\varepsilon^{\mu \nu \lambda \rho} \bar{T}^{a b c}{ }_{\mu} u_{a}{ }_{\nu}{ }_{\nu} u_{d}{ }^{e}{ }_{\lambda} h_{e}{ }^{f}{ }_{\rho}^{\sigma} T_{b c f \sigma}+$ H.c. & & 251 & $i \bar{T}^{a b c \mu} u_{a}{ }^{d \nu} u_{b}{ }^{e \lambda} \chi_{-d}{ }^{f} \gamma_{5} \gamma_{\lambda} D_{\nu} T_{c e f \mu}+$ H.c. & 285 & 506 \\
\hline$\varepsilon^{\mu \nu \lambda \rho} \bar{T}^{a b c}{ }_{\mu} u_{a}{ }_{\nu}{ }_{\nu} u^{e f}{ }_{\lambda} h_{d e \rho}{ }^{\sigma} T_{b c f \sigma}+$ H.c. & & 252 & $i \bar{T}^{a b c \mu} u_{a}{ }^{d \nu} u_{d}{ }^{e \lambda} \chi_{-b}{ }^{f} \gamma_{5} \gamma_{\nu} D_{\lambda} T_{c e f \mu}+$ H.c. & 286 & 507 \\
\hline$\varepsilon^{\mu \nu \lambda \rho} \bar{T}^{a b c}{ }_{\mu} u_{a}{ }^{d}{ }_{\nu} u^{e f}{ }_{\lambda} h_{f e \rho}{ }^{\sigma} T_{b c d \sigma}+$ H.c. & & 253 & $i \bar{T}^{a b c \mu} u_{a}{ }^{d \nu} u_{d}^{e \lambda} \chi_{-e}{ }^{f} \gamma_{5} \gamma_{\nu} D_{\lambda} T_{b c f \mu}+$ H.c. & & 508 \\
\hline$\varepsilon^{\mu \nu \lambda \rho} \bar{T}^{a b c}{ }_{\mu} u_{a}{ }^{d}{ }_{\nu} u_{b}{ }^{e \sigma} h_{d}{ }^{f}{ }_{\lambda \sigma} T_{c e f \rho}+$ H.c. & 90 & 254 & $i \bar{T}^{a b c \mu} u_{a}{ }^{d \nu} u_{d}^{e \lambda} \chi_{-e}{ }^{f} \gamma_{5} \gamma_{\lambda} D_{\nu} T_{b c f \mu}+$ H.c. & & 509 \\
\hline$\varepsilon^{\mu \nu \lambda \rho} \bar{T}^{a b c}{ }_{\mu} u_{a}{ }^{d}{ }_{\nu} u_{d}{ }^{e \sigma} h_{e}{ }_{\lambda \sigma} T_{b c f \rho}+$ H.c. & & 255 & $i \bar{T}^{a b c \mu} u_{a}{ }^{d \nu} u^{e f \lambda} \chi_{-d e} \gamma_{5} \gamma_{\nu} D_{\lambda} T_{b c f \mu}+$ H.c. & & 510 \\
\hline $\bar{T}^{a b c \mu} f_{-a \mu}{ }^{\mu}{ }^{\nu} f_{-b \nu}{ }^{\lambda}{ }^{\lambda} T_{c d e \lambda}$ & 91 & 256 & $i \bar{T}^{a b c \mu} u^{d e \nu} u_{e}^{f \lambda} \chi_{-f d} \gamma_{5} \gamma_{\nu} D_{\lambda} T_{a b c \mu}+$ H.c. & & 511 \\
\hline $\bar{T}^{a b c \mu} f_{-a}{ }^{\mu \nu \lambda} f_{-b}{ }^{e}{ }_{\nu \lambda} T_{c d e \mu}$ & 92 & 257 & $i \bar{T}^{a b c \mu} u_{a}{ }^{d \nu} u_{d}^{e \lambda} \chi_{-, s} \gamma_{5} \gamma_{\nu} D_{\lambda} T_{b c e \mu}+$ H.c. & 287 & 512 \\
\hline $\bar{T}^{a b c \mu} f_{-a}{ }^{d}{ }^{\nu} f_{-d}{ }^{e}{ }_{\nu}^{\lambda} T_{b c e \lambda}$ & 93 & 258 & $i \varepsilon^{\mu \nu \lambda \rho} \bar{T}^{a b c}{ }_{\mu} u_{a}{ }^{d}{ }_{\nu} u_{b}{ }^{e} \lambda \chi_{-d}{ }^{f} T_{c e f \rho}+$ H.c. & 288 & 513 \\
\hline $\bar{T}^{a b c \mu} f_{-a}{ }^{d \nu \lambda} f_{-d}{ }^{e} \mu \nu T_{b c e \lambda}$ & 94 & 259 & $i \varepsilon^{\mu \nu \lambda \rho} \bar{T}^{a b c}{ }_{\mu} u_{a}{ }^{d}{ }_{\nu} u_{d}{ }^{e}{ }_{\lambda} \chi_{-b}{ }^{f} T_{c e f \rho}$ & 289 & 514 \\
\hline $\bar{T}^{a b c \mu} f_{-a}{ }^{d \nu \lambda} f_{-d}{ }^{e \nu \lambda} T_{b c e \mu}$ & 95 & 260 & $i \varepsilon^{\mu \nu \lambda \rho} \bar{T}^{a b c}{ }_{\mu} u_{a}{ }^{d}{ }_{\nu} u_{d}{ }^{e} \lambda \chi_{-e}{ }^{f} T_{b c f \rho}+$ H.c. & & 515 \\
\hline $\bar{T}^{a b c \mu} f_{-}{ }^{d e}{ }_{\mu}^{\nu} f_{-e d \nu}^{\lambda} T_{a b c \lambda}$ & & 261 & $i \varepsilon^{\mu \nu \lambda \rho} \bar{T}^{a b c}{ }_{\mu} u_{a}{ }^{d}{ }_{\nu} u^{e f}{ }_{\lambda} \chi_{-d e} T_{b c f \rho}$ & & 516 \\
\hline $\bar{T}^{a b c \mu} f_{-}{ }^{d e \nu \lambda} f_{-e d \nu \lambda} T_{a b c \mu}$ & & 262 & $i \varepsilon^{\mu \nu \lambda \rho} \bar{T}^{a b c}{ }_{\mu} u^{d e}{ }_{\nu} u_{e}{ }_{\lambda}^{f} \chi_{-f d} T_{a b c \rho}$ & & 517 \\
\hline $\bar{T}^{a b c \mu} f_{-a}{ }^{d}{ }^{\nu} f_{-b}{ }^{e \lambda \rho} D_{\nu \lambda} T_{c d e \rho}$ & 96 & 263 & $i \varepsilon^{\mu \nu \lambda \rho} \bar{T}^{a b c}{ }_{\mu} u_{a}{ }^{d}{ }_{\nu} u_{d}{ }^{e} \lambda \chi_{-, s} T_{b c e \rho}$ & 290 & 518 \\
\hline $\bar{T}^{a b c \mu} f_{-a}{ }^{\mu \nu \lambda} f_{-b}{ }^{e}{ }^{\rho} D_{\lambda \rho} T_{c d e \mu}$ & 97 & 264 & $i \bar{T}^{a b c \mu} f_{-a}^{d}{ }_{\mu}^{\nu} \chi_{-d}^{e} T_{b c e \nu}+$ H.c. & 291 & 519 \\
\hline $\bar{T}^{a b c \mu} f_{-a}{ }^{\mu}{ }_{\mu}^{\nu} f_{-d}{ }^{e \lambda \rho} D_{\nu \lambda} T_{b c e \rho}$ & 98 & 265 & $i \bar{T}^{a b c \mu} h_{a}{ }^{d}{ }_{\mu}{ }^{\nu} \chi_{-b}{ }^{e} T_{c d e \nu}$ & 292 & 520 \\
\hline $\bar{T}^{a b c \mu} f_{-a}{ }^{\mu \nu \lambda} f_{-d}{ }^{e}{ }^{\rho} D_{\nu \rho} T_{b c e \lambda}$ & 99 & 266 & $i \bar{T}^{a b c \mu} h_{a}{ }^{d}{ }_{\mu}^{\nu} \chi_{-d}^{e} T_{b c e \nu}+$ H.c. & 293 & 521 \\
\hline $\bar{T}^{a b c \mu} f_{-a}{ }^{d \nu \lambda} f_{-d}{ }^{e}{ }^{\rho}{ }^{\rho} D_{\lambda \rho} T_{b c e \mu}$ & 100 & 267 & $i \bar{T}^{a b c \mu} h^{d e}{ }_{\mu}^{\nu} \chi_{-e d} T_{a b c \nu}$ & & 522 \\
\hline $\bar{T}^{a b c \mu} f_{-}{ }^{d e}{ }_{\mu}^{\nu} f_{-e d}{ }^{\lambda \rho} D_{\nu \lambda} T_{a b c \rho}$ & & 268 & $i \bar{T}^{a b c \mu} h_{a}^{d \nu \lambda} \chi_{-b}^{e} D_{\nu \lambda} T_{c d e \mu}$ & 294 & 523 \\
\hline $\bar{T}^{a b c \mu} f_{-}{ }^{\operatorname{de\nu } \lambda} f_{-e d \nu}{ }^{\rho} D_{\lambda \rho} T_{a b c \mu}$ & & 269 & $i \bar{T}^{a b c \mu} h_{a}{ }^{d \nu \lambda} \chi_{-d}^{e} D_{\nu \lambda} T_{b c e \mu}+$ H.c. & 295 & 524 \\
\hline
\end{tabular}


TABLE V. (Continued)

\begin{tabular}{|c|c|c|c|c|c|}
\hline$O_{n}^{\left(N_{f}, 4\right)}$ & $S U(2)$ & $S U(3)$ & $O_{n}^{\left(N_{f}, 4\right)}$ & $S U(2)$ & $S U(3)$ \\
\hline$i \bar{T}^{a b c \mu} f_{-a}{ }^{d \mu}{ }^{\nu} f_{-b}{ }^{e \lambda \rho} \sigma_{\nu \lambda} T_{c d e \rho}$ & 101 & 270 & $i \bar{T}^{a b c \mu} h^{d e \nu \lambda} \chi_{-e d} D_{\nu \lambda} T_{a b c \mu}$ & & 525 \\
\hline$i \bar{T}^{a b c \mu} f_{-a}{ }^{\lambda}{ }^{\nu} f_{-d}{ }^{e \lambda \rho} \sigma_{\nu \lambda} T_{b c e \rho}$ & 102 & 271 & $i \bar{T}^{a b c \mu} h_{a}{ }_{\mu}{ }^{\nu} \chi_{-, s} T_{b c d \nu}$ & 296 & 526 \\
\hline$i \bar{T}^{a b c \mu} f_{-} d{ }_{\mu}^{\nu} f_{-e d}{ }^{\lambda \rho} \sigma_{\nu \lambda} T_{a b c \rho}$ & & 272 & $i \bar{T}^{a b c \mu} h_{a}^{d \nu \lambda} \chi_{-, s} D_{\nu \lambda} T_{b c d \mu}$ & 297 & 527 \\
\hline $\bar{T}^{a b c \mu} h_{a}{ }^{d}{ }_{\mu}^{\nu} f_{-b}{ }^{e}{ }_{\nu}^{\lambda} T_{c d e \lambda}+$ H.c. & 103 & 273 & $i \bar{T}^{a b c \mu} u_{a}{ }^{d \nu} \nabla_{\nu} \chi_{-b}{ }^{e} T_{c d e \mu}$ & 298 & 528 \\
\hline $\bar{T}^{a b c \mu} h_{a}{ }^{d}{ }^{\nu} f_{-d}{ }^{e}{ }_{\nu}^{\lambda} T_{b c e \lambda}+$ H.c. & 104 & 274 & $i \bar{T}^{a b c \mu} u_{a}{ }^{d \nu} \nabla_{\nu} \chi_{-d}{ }^{e} T_{b c e \mu}+$ H.c. & 299 & 529 \\
\hline $\bar{T}^{a b c \mu} h_{a}{ }^{d \nu \lambda} f_{-d}{ }^{e}{ }_{\mu \nu} T_{b c e \lambda}+$ H.c. & 105 & 275 & $i \bar{T}^{a b c \mu} u^{d e \nu} \nabla_{\nu} \chi_{-e d} T_{a b c \mu}$ & & 530 \\
\hline $\bar{T}^{a b c \mu} h^{d e}{ }_{\mu}^{\nu} f_{-e d \nu}{ }^{\lambda} T_{a b c \lambda}+$ H.c. & & 276 & $i \bar{T}^{a b c \mu} u_{a}^{d \nu} \nabla_{\nu} \chi_{-, s} T_{b c d \mu}$ & 300 & 531 \\
\hline $\bar{T}^{a b c \mu} h_{a}{ }^{\mu}{ }_{\mu}^{\nu} f_{-b}{ }^{e \lambda \rho} D_{\nu \lambda} T_{c d e \rho}+$ H.c. & 106 & 277 & $\bar{T}^{a b c \mu} f_{+a}{ }^{d \nu \lambda} \chi_{-b}{ }^{e} \gamma_{5} \gamma_{\nu} D_{\lambda} T_{c d e \mu}$ & 301 & 532 \\
\hline $\bar{T}^{a b c \mu} h_{a}^{d \nu \lambda} f_{-b}{ }^{e}{ }_{\nu}^{\rho} D_{\lambda \rho} T_{c d e \mu}$ & 107 & 278 & $\bar{T}^{a b c \mu} f_{+a}{ }^{d \nu \lambda} \chi_{-d}{ }^{e} \gamma_{5} \gamma_{\nu} D_{\lambda} T_{b c e \mu}+$ H.c. & 302 & 533 \\
\hline $\bar{T}^{a b c \mu} h_{a}{ }^{d}{ }_{\mu}^{\nu} f_{-d}{ }^{e \lambda \rho} D_{\nu \lambda} T_{b c e \rho}+$ H.c. & 108 & 279 & $\bar{T}^{a b c \mu} f_{s,+}{ }^{\nu \lambda} \chi_{-a}{ }^{d} \gamma_{5} \gamma_{\nu} D_{\lambda} T_{b c d \mu}$ & 303 & \\
\hline $\bar{T}^{a b c \mu} h_{a}{ }^{d \nu \lambda} f_{-d \mu}{ }^{\rho}{ }^{\rho} D_{\nu \lambda} T_{b c e \rho}+$ H.c. & 109 & 280 & $\bar{T}^{a b c \mu} f_{+}{ }^{d e \nu \lambda} \chi_{-e d} \gamma_{5} \gamma_{\nu} D_{\lambda} T_{a b c \mu}$ & & 534 \\
\hline $\bar{T}^{a b c \mu} h_{a}{ }^{d \nu \lambda} f_{-d}{ }^{e}{ }^{\rho} D_{\lambda \rho} T_{b c e \mu}+$ H.c. & 110 & 281 & $\bar{T}^{a b c \mu} f_{+a}{ }^{d \nu \lambda} \chi_{-, s} \gamma_{5} \gamma_{\nu} D_{\lambda} T_{b c d \mu}$ & 304 & 535 \\
\hline $\bar{T}^{a b c \mu} h^{d e}{ }_{\mu}^{\nu} f_{-e d}{ }^{\lambda \rho} D_{\nu \lambda} T_{a b c \rho}+$ H.c. & & 282 & $\bar{T}^{a b c \mu} f_{s,+}{ }^{\nu \lambda} \chi_{-, s} \gamma_{5} \gamma_{\nu} D_{\lambda} T_{a b c \mu}$ & 305 & \\
\hline $\bar{T}^{a b c \mu} h^{d e \nu \lambda} f_{-e d \nu}^{\rho} D_{\lambda \rho} T_{a b c \mu}$ & & 283 & $\varepsilon^{\mu \nu \lambda \rho} \bar{T}^{a b c}{ }_{\mu} f_{+a}{ }^{d}{ }_{\nu \lambda} \chi_{-b}{ }^{e} T_{c d e \rho}$ & 306 & 536 \\
\hline$i \bar{T}^{a b c \mu} h_{a}{ }^{d}{ }_{\mu}^{\nu} f_{-d}{ }^{e \lambda \rho} \sigma_{\nu \lambda} T_{b c e \rho}+$ H.c. & 111 & 284 & $\varepsilon^{\mu \nu \lambda \rho} \bar{T}^{a b c}{ }_{\mu} f_{+a}{ }^{d}{ }_{\nu \lambda} \chi_{-d}{ }^{e} T_{b c e \rho}+$ H.c. & 307 & 537 \\
\hline $\bar{T}^{a b c \mu} h_{a}{ }_{\mu}^{d}{ }^{\nu} h_{b}{ }_{\nu}{ }_{\nu}^{\lambda} T_{c d e \lambda}$ & 112 & 285 & $\varepsilon^{\mu \nu \lambda \rho} \bar{T}_{\mu}^{a b c} f_{s,+\nu \lambda \chi_{-a}{ }^{d} T_{b c d \rho}}$ & 308 & \\
\hline $\bar{T}^{a b c \mu} h_{a}{ }^{d \nu \lambda} h_{b}{ }^{e}{ }_{\nu \lambda} T_{c d e \mu}$ & 113 & 286 & $\varepsilon^{\mu \nu \lambda \rho} \bar{T}^{a b c}{ }_{\mu} f_{+}{ }^{d e}{ }_{\nu \lambda} \chi_{-e d} T_{a b c \rho}$ & & 538 \\
\hline $\bar{T}^{a b c \mu} h_{a \mu}{ }^{d}{ }^{\nu} h_{d \nu}{ }^{e}{ }^{\lambda} T_{b c e \lambda}$ & 114 & 287 & $\varepsilon^{\mu \nu \lambda \rho} \bar{T}_{\mu}^{a b c} f_{+a}{ }_{\nu \lambda} \chi_{-, s} T_{b c d \rho}$ & 309 & 539 \\
\hline $\bar{T}^{a b c \mu} h_{a}^{d \nu \lambda} h_{d}^{e}{ }^{e} T_{b c e \mu}$ & 115 & 288 & $\varepsilon^{\mu \nu \lambda \rho} \bar{T}_{\mu}^{a b c} f_{s,+\nu \lambda \chi_{-, s} T_{a b c \rho}}$ & 310 & \\
\hline $\bar{T}^{a b c \mu} h^{d e}{ }_{\mu}^{\nu} h_{e d \nu}{ }^{\lambda} T_{a b c \lambda}$ & & 289 & $\bar{T}^{a b c \mu} \chi_{-a}{ }^{d} \chi_{-b}{ }^{e} T_{c d e \mu}$ & 311 & 540 \\
\hline $\bar{T}^{a b c \mu} h^{d e \nu \lambda} h_{e d \nu \lambda} T_{a b c \mu}$ & & 290 & $\bar{T}^{a b c \mu} \chi_{-a}{ }^{d} \chi_{-d}{ }^{e} T_{b c e \mu}$ & & 541 \\
\hline $\bar{T}^{a b c \mu} h_{a}{ }^{d}{ }_{\mu}{ }^{\nu} h_{b}{ }^{e \lambda \rho} D_{\nu \lambda} T_{c d e \rho}$ & 116 & 291 & $\bar{T}^{a b c \mu} \chi_{-}{ }^{d e} \chi_{-e d} T_{a b c \mu}$ & & 542 \\
\hline $\bar{T}^{a b c \mu} h_{a}{ }^{d \nu \lambda} h_{b}{ }^{e}{ }_{\nu}{ }^{\rho} D_{\lambda \rho} T_{c d e \mu}$ & 117 & 292 & $\bar{T}^{a b c \mu} \chi_{-a}{ }^{d} \chi_{-, s} T_{b c d \mu}$ & 312 & 543 \\
\hline $\bar{T}^{a b c \mu} h_{a}{ }^{d}{ }_{\mu}{ }^{\nu} h_{d}{ }^{e \lambda \rho} D_{\nu \lambda} T_{b c e \rho}$ & 118 & 293 & $\bar{T}^{a b c \mu}\left\langle F_{L \mu}{ }^{\nu} F_{L \nu}{ }^{\lambda}\right\rangle T_{a b c \lambda}+$ H.c. & 313 & 544 \\
\hline $\bar{T}^{a b c \mu} h_{a}{ }^{d \nu \lambda} h_{d}{ }^{e}{ }_{\nu}^{\rho} D_{\lambda \rho} T_{b c e \mu}$ & 119 & 294 & $\bar{T}^{a b c \mu}\left\langle F_{L}^{\nu \lambda} F_{L \nu \lambda}\right\rangle T_{a b c \mu}+$ H.c. & 314 & 545 \\
\hline $\bar{T}^{a b c \mu} h^{d e}{ }_{\mu}{ }^{\nu} h_{e d}{ }^{\lambda \rho} D_{\nu \lambda} T_{a b c \rho}$ & & 295 & $\bar{T}^{a b c \mu}\left\langle F_{L \mu}{ }^{\nu} F_{L}{ }^{\lambda \rho}\right\rangle D_{\nu \lambda} T_{a b c \rho}+$ H.c. & 315 & 546 \\
\hline $\bar{T}^{a b c \mu} h^{d e \nu \lambda} h_{e d \nu}{ }^{\rho} D_{\lambda \rho} T_{a b c \mu}$ & & 296 & $\bar{T}^{a b c \mu}\left\langle F_{L}^{\nu \lambda} F_{L \nu}{ }^{\rho}\right\rangle D_{\lambda \rho} T_{a b c \mu}+$ H.c. & 316 & 547 \\
\hline $\bar{T}^{a b c \mu} h_{a}{ }^{d \nu \lambda} h_{b}{ }^{e \rho \sigma} D_{\nu \lambda \rho \sigma} T_{c d e \mu}$ & 120 & 297 & $\bar{T}^{a b c \mu}\left\langle\chi \chi^{\dagger}\right\rangle T_{a b c \mu}$ & 317 & 548 \\
\hline $\bar{T}^{a b c \mu} h_{a}{ }^{d \nu \lambda} h_{d}{ }^{e \rho \sigma} D_{\nu \lambda \rho \sigma} T_{b c e \mu}$ & 121 & 298 & $\bar{T}^{a b c \mu} \operatorname{det} \chi T_{a b c \mu}+$ H.c. & 318 & \\
\hline
\end{tabular}

TABLE VI. Terms in the $\mathcal{O}\left(p^{3}\right)$ meson-octet-decuplet and $\pi N \Delta$ chiral Lagrangians, where $P_{n}^{\left(N_{f}, 3\right)}$ is defined in Eqs. (64) and (66). For the $S U(2)$ case, the form needs to be changed; see the sentences around Eq. (67).

\begin{tabular}{|c|c|c|c|c|c|}
\hline$P_{n}^{\left(N_{f}, 3\right)}$ & $S U(2)$ & $S U(3)$ & $P_{n}^{\left(N_{f}, 3\right)}$ & $S U(2)$ & $S U(3)$ \\
\hline$\epsilon^{a b c} \bar{B}_{a}{ }^{d} u_{b}{ }^{e \mu} u_{c}{ }^{f}{ }_{\mu} u_{e}{ }^{g \nu} T_{A, n, d f g \nu}$ & 1 & 1 & $\epsilon^{a b c} \bar{B}_{a}^{d} u_{b}^{e \mu} f_{-e}{ }^{f \nu \lambda} \gamma_{5} \gamma_{\nu} D_{\lambda} T_{A, n, c d f \mu}$ & & 36 \\
\hline$\epsilon^{a b c} \bar{B}_{a}{ }^{d} u_{b}{ }^{e \mu} u_{c}{ }^{f \nu} u_{d}^{g}{ }_{\mu} T_{A, n, e f g \nu}$ & 2 & 2 & $\epsilon^{a b c} \bar{B}_{a}^{d} u^{e f \mu} f_{-b e}{ }^{\nu \lambda} \gamma_{5} \gamma_{\mu} D_{\nu} T_{A, n, c d f \lambda}$ & & 37 \\
\hline$\epsilon^{a b c} \bar{B}_{a}{ }^{d} u_{b}{ }^{e \mu} u_{c}{ }^{f \nu} u_{e}{ }_{\mu}{ }_{\mu} T_{A, n, d f g \nu}$ & & 3 & $\epsilon^{a b c} \bar{B}_{a}{ }^{d} u^{e f \mu} f_{-b e}{ }^{\nu \lambda} \gamma_{5} \gamma_{\nu} D_{\mu} T_{A, n, c d f \lambda}$ & & 38 \\
\hline$\epsilon^{a b c} \bar{B}_{a}{ }^{d} u_{b}{ }^{e \mu} u_{c}{ }^{f \nu} u_{e}{ }^{g}{ }_{\nu} T_{A, n, d f g \mu}$ & 3 & 4 & $\epsilon^{a b c} \bar{B}_{a}{ }^{d} u^{e f \mu} f_{-b e}{ }^{\nu \lambda} \gamma_{5} \gamma_{\nu} D_{\lambda} T_{A, n, c d f \mu}$ & & 39 \\
\hline$\epsilon^{a b c} \bar{B}_{a}{ }^{d} u_{b}{ }^{e \mu} u_{d}{ }^{f}{ }_{\mu} u_{e}{ }^{g \nu} T_{A, n, c f g \nu}$ & & 5 & $\epsilon^{a b c} \bar{B}_{a}{ }^{d} u_{b}{ }^{e \mu} h_{c}{ }^{f \nu \lambda} \gamma_{5} \gamma_{\mu} D_{\nu} T_{A, n, \operatorname{def} \lambda}$ & 15 & 40 \\
\hline$\epsilon^{a b c} \bar{B}_{a}{ }^{d} u_{b}{ }^{e \mu} u_{d}{ }^{f \nu} u_{e}{ }_{\mu}{ }_{\mu} T_{A, n, c f g \nu}$ & & 6 & $\epsilon^{a b c} \bar{B}_{a}{ }^{d} u_{b}{ }^{e \mu} h_{d}{ }^{f \nu \lambda} \gamma_{5} \gamma_{\mu} D_{\nu} T_{A, n, c e f \lambda}$ & 16 & 41 \\
\hline$\epsilon^{a b c} \bar{B}_{a}{ }^{d} u_{b}{ }^{e \mu} u_{d}{ }^{f \nu} u_{e}{ }_{\nu}{ }_{\nu} T_{A, n, c f g \mu}$ & & 7 & $\epsilon^{a b c} \bar{B}_{a}{ }^{d} u_{b}{ }^{e \mu} h_{e}{ }^{f \nu \lambda} \gamma_{5} \gamma_{\mu} D_{\nu} T_{A, n, c d f \lambda}$ & & 42 \\
\hline$\epsilon^{a b c} \bar{B}_{a}{ }^{d} u_{b}{ }^{e \mu} u_{e}{ }^{f}{ }_{\mu} u_{f}^{g \nu} T_{A, n, c d g \nu}$ & & 8 & $\epsilon^{a b c} \bar{B}_{a}{ }^{d} u_{b}{ }^{e \mu} h_{e}{ }^{f \nu \lambda} \gamma_{5} \gamma_{\nu} D_{\mu} T_{A, n, c d f \lambda}$ & & 43 \\
\hline$\epsilon^{a b c} \bar{B}_{a}^{d} u_{b}{ }^{e \mu} u_{e}{ }^{f \nu} u_{f}^{g}{ }_{\mu} T_{A, n, c d g \nu}$ & & 9 & $\varepsilon^{\mu \nu \lambda \rho} \epsilon^{a b c} \bar{B}_{a}{ }^{d} u_{b}{ }_{\mu} f_{-c}{ }^{f}{ }_{\nu \lambda} T_{A, n, \operatorname{def} \rho}$ & 17 & 44 \\
\hline$\epsilon^{a b c} \bar{B}_{a}^{d} u_{b}^{e \mu} u_{e}^{f \nu} u_{f}^{g}{ }_{\nu} T_{A, n, c d g \mu}$ & & 10 & $\varepsilon^{\mu \nu \lambda \rho} \epsilon^{a b c} \bar{B}_{a}{ }^{d} u_{b}{ }_{\mu}{ }_{\mu} f_{-d}{ }^{f}{ }_{\nu \lambda} T_{A, n, c e f \rho}$ & 18 & 45 \\
\hline$\epsilon^{a b c} \bar{B}_{a}{ }^{d} u_{b}{ }^{e \mu} u_{c}{ }^{f \nu} u_{d}{ }^{g \lambda} D_{\mu \lambda} T_{A, n, e f g \nu}$ & 4 & 11 & $\varepsilon^{\mu \nu \lambda \rho} \epsilon^{a b c} \bar{B}_{a}{ }^{d} u_{b}{ }^{e}{ }_{\mu} f_{-e}{ }_{\nu \lambda \lambda} T_{A, n, c d f \rho}$ & & 46 \\
\hline$\epsilon^{a b c} \bar{B}_{a}{ }^{d} u_{b}{ }^{e \mu} u_{c}{ }^{f \nu} u_{e}{ }^{g \lambda} D_{\mu \nu} T_{A, n, d f g \lambda}$ & 5 & 12 & $\varepsilon^{\mu \nu \lambda \rho} \epsilon^{a b c} \bar{B}_{a}{ }^{d} u^{e f}{ }_{\mu} f_{-b e \nu \lambda} T_{A, n, c d f \rho}$ & & 47 \\
\hline
\end{tabular}


TABLE VI. (Continued)

\begin{tabular}{|c|c|c|c|c|c|}
\hline$P_{n}^{\left(N_{f}, 3\right)}$ & $S U(2)$ & $S U(3)$ & $P_{n}^{\left(N_{f}, 3\right)}$ & $S U(2)$ & $S U(3)$ \\
\hline$\epsilon^{a b c} \bar{B}_{a}{ }^{d} u_{b}{ }^{e \mu} u_{c}{ }^{f \nu} u_{e}{ }^{g \lambda} D_{\mu \lambda} T_{A, n, d f g \nu}$ & & 13 & $\epsilon^{a b c} \bar{B}_{a}{ }^{d} \nabla^{\mu} f_{-b}{ }^{e}{ }^{\nu} T_{A, n, c d e \nu}$ & 19 & 48 \\
\hline$\epsilon^{a b c} \bar{B}_{a}{ }^{d} u_{b}{ }^{e \mu} u_{c}{ }^{f \nu} u_{e}{ }^{g \lambda} D_{\nu \lambda} T_{A, n, d f g \mu}$ & 6 & 14 & $i \epsilon^{a b c} \bar{B}_{a}{ }^{d} \nabla^{\mu} f_{-b}{ }^{e \nu \lambda} \sigma_{\mu \nu} T_{A, n, c d e \lambda}$ & 20 & 49 \\
\hline$\epsilon^{a b c} \bar{B}_{a}{ }^{d} u_{b}{ }^{e \mu} u_{d}{ }^{f \nu} u_{e}{ }^{g \lambda} D_{\mu \nu} T_{A, n, c f g \lambda}$ & & 15 & $i \epsilon^{a b c} \bar{B}_{a}{ }^{d} f_{+b}{ }^{e \mu \nu} u_{c}{ }^{f}{ }_{\mu} T_{A, n, d e f \nu}$ & 21 & 50 \\
\hline$\epsilon^{a b c} \bar{B}_{a}{ }^{d} u_{b}{ }^{e \mu} u_{d}{ }^{f \nu} u_{e}{ }^{g \lambda} D_{\mu \lambda} T_{A, n, c f g \nu}$ & & 16 & $i \epsilon^{a b c} \bar{B}_{a}^{d} f_{+b}{ }^{e \mu \nu} u_{d}^{f}{ }_{\mu} T_{A, n, c e f \nu}$ & 22 & 51 \\
\hline$\epsilon^{a b c} \bar{B}_{a}{ }^{d} u_{b}{ }^{e \mu} u_{d}{ }^{f \nu} u_{e}{ }^{g \lambda} D_{\nu \lambda} T_{A, n, c f g \mu}$ & & 17 & $i \epsilon^{a b c} \bar{B}_{a}{ }^{d} f_{+b}{ }^{e \mu \nu} u_{e}{ }^{f_{\mu}^{\prime}} T_{A, n, c d f \nu}$ & & 52 \\
\hline$\epsilon^{a b c} \bar{B}_{a}^{d} u_{b}{ }^{e \mu} u_{e}{ }^{f \nu} u_{f}^{g \lambda} D_{\mu \nu} T_{A, n, c d g \lambda}$ & & 18 & $i \epsilon^{a b c} \bar{B}_{a}^{d} f_{+}{ }^{e f \mu \nu} u_{b e \mu} T_{A, n, c d f \nu}$ & & 53 \\
\hline$\epsilon^{a b c} \bar{B}_{a}{ }^{d} u_{b}{ }^{e \mu} u_{e}{ }^{f \nu} u_{f}^{g \lambda} D_{\mu \lambda} T_{A, n, c d g \nu}$ & & 19 & $i \epsilon^{a b c} \bar{B}_{a}{ }^{d} f_{+b}{ }^{e \mu \nu} u_{c}{ }^{f \lambda} D_{\mu \lambda} T_{A, n, d e f \nu}$ & 23 & 54 \\
\hline$\epsilon^{a b c} \bar{B}_{a}{ }^{d} u_{b}{ }^{e \mu} u_{e}{ }^{f \nu} u_{f}^{g \lambda} D_{\nu \lambda} T_{A, n, c d g \mu}$ & & 20 & $i \epsilon^{a b c} \bar{B}_{a}{ }^{d} f_{+b}{ }^{e \mu \nu} u_{d}{ }^{f \lambda} D_{\mu \lambda} T_{A, n, c e f \nu}$ & 24 & 55 \\
\hline$i \epsilon^{a b c} \bar{B}_{a}{ }^{d} u_{b}{ }^{e \mu} u_{c}{ }^{f \nu} u_{d}{ }^{g \lambda} \sigma_{\mu \nu} T_{A, n, e f g \lambda}$ & 7 & 21 & $i \epsilon^{a b c} \bar{B}_{a}^{d} f_{+b}^{e \mu \nu} u_{e}^{f \lambda} D_{\mu \lambda} T_{A, n, c d f \nu}$ & & 56 \\
\hline$i \epsilon^{a b c} \bar{B}_{a}{ }^{d} u_{b}{ }^{e \mu} u_{c}{ }^{f \nu} u_{e}{ }^{g \lambda} \sigma_{\mu \nu} T_{A, n, d f g \lambda}$ & 8 & 22 & $i \epsilon^{a b c} \bar{B}_{a}^{d} f_{+}{ }^{e f \mu \nu} u_{b e}{ }^{\lambda} D_{\mu \lambda} T_{A, n, c d f \nu}$ & & 57 \\
\hline$i \epsilon^{a b c} \bar{B}_{a}{ }^{d} u_{b}{ }^{e \mu} u_{c}{ }^{f \nu} u_{e}{ }^{g \lambda} \sigma_{\mu \lambda} T_{A, n, d f g \nu}$ & & 23 & $\epsilon^{a b c} \bar{B}_{a}^{d} f_{+b}{ }^{e \mu \nu} u_{c}{ }^{f \lambda} \sigma_{\mu \nu} T_{A, n, \operatorname{def} \lambda}$ & 25 & 58 \\
\hline$i \epsilon^{a b c} \bar{B}_{a}{ }^{d} u_{b}{ }^{e \mu} u_{d}{ }^{f \nu} u_{e}{ }^{g \lambda} \sigma_{\mu \nu} T_{A, n, c f g \lambda}$ & & 24 & $\epsilon^{a b c} \bar{B}_{a}^{d} f_{+b}{ }^{e \mu \nu} u_{d}^{f \lambda} \sigma_{\mu \nu} T_{A, n, c e f \lambda}$ & 26 & 59 \\
\hline$i \epsilon^{a b c} \bar{B}_{a}{ }^{d} u_{b}{ }^{e \mu} u_{d}{ }^{f \nu} u_{e}{ }^{g \lambda} \sigma_{\mu \lambda} T_{A, n, c f g \nu}$ & & 25 & $i \epsilon^{a b c} \bar{B}_{a}{ }^{d} f_{s,+}{ }^{\mu \nu} u_{b}{ }^{e}{ }_{\mu} T_{A, n, c d e \nu}$ & 27 & \\
\hline$i \epsilon^{a b c} \bar{B}_{a}{ }^{d} u_{b}{ }^{e \mu} u_{e}{ }^{f \nu} u_{f}^{g \lambda} \sigma_{\mu \nu} T_{A, n, c d g \lambda}$ & & 26 & $i \epsilon^{a b c} \bar{B}_{a}{ }^{d} f_{s,+}{ }^{\mu \nu} u_{b}{ }^{e \lambda} D_{\mu \lambda} T_{A, n, c d e \nu}$ & 28 & \\
\hline$i \epsilon^{a b c} \bar{B}_{a}{ }^{d} u_{b}{ }^{e \mu} u_{e}{ }^{f \nu} u_{f}^{g \lambda} \sigma_{\mu \lambda} T_{A, n, c d g \nu}$ & & 27 & $\epsilon^{a b c} \bar{B}_{a}^{d} f_{s,+}^{\mu \nu} u_{b}^{e \lambda} \sigma_{\mu \nu} T_{A, n, c d e \lambda}$ & 29 & \\
\hline$\epsilon^{a b c} \bar{B}_{a}{ }^{d} u_{b}{ }^{e \mu} f_{-c}{ }^{f \nu \lambda} \gamma_{5} \gamma_{\mu} D_{\nu} T_{A, n, \text { def } \lambda}$ & 9 & 28 & $\epsilon^{a b c} \bar{B}_{a}^{d} f_{+b}{ }^{e \mu \nu} u_{e}^{f \lambda} \sigma_{\mu \nu} T_{A, n, c d f \lambda}$ & & 60 \\
\hline$\epsilon^{a b c} \bar{B}_{a}{ }^{d} u_{b}{ }^{e \mu} f_{-c}{ }^{f \nu \lambda} \gamma_{5} \gamma_{\nu} D_{\mu} T_{A, n, \text { def } \lambda}$ & 10 & 29 & $\epsilon^{a b c} \bar{B}_{a}^{d} f_{+}{ }^{e f \mu \nu} u_{b e}{ }^{\lambda} \sigma_{\mu \nu} T_{A, n, c d f \lambda}$ & & 61 \\
\hline$\epsilon^{a b c} \bar{B}_{a}{ }^{d} u_{b}{ }^{e \mu} f_{-c}{ }^{f \nu \lambda} \gamma_{5} \gamma_{\nu} D_{\lambda} T_{A, n, \text { def } \mu}$ & 11 & 30 & $\epsilon^{a b c} \bar{B}_{a}{ }^{d} u_{b}{ }^{e \mu} \chi_{+c}{ }^{f} T_{A, n, \text { def } \mu}$ & 30 & 62 \\
\hline$\epsilon^{a b c} \bar{B}_{a}{ }^{d} u_{b}{ }^{e \mu} f_{-d}{ }^{f \nu \lambda} \gamma_{5} \gamma_{\mu} D_{\nu} T_{A, n, c e f \lambda}$ & 12 & 31 & $\epsilon^{a b c} \bar{B}_{a}^{d} u_{b}{ }^{e \mu} \chi_{+d}{ }^{f} T_{A, n, c e f \mu}$ & 31 & 63 \\
\hline$\epsilon^{a b c} \bar{B}_{a}{ }^{d} u_{b}{ }^{e \mu} f_{-d}{ }^{f \nu \lambda} \gamma_{5} \gamma_{\nu} D_{\mu} T_{A, n, c e f \lambda}$ & 13 & 32 & $\epsilon^{a b c} \bar{B}_{a}{ }^{d} u_{b}{ }^{e \mu} \chi_{+e}{ }^{f} T_{A, n, c d f \mu}$ & & 64 \\
\hline$\epsilon^{a b c} \bar{B}_{a}{ }^{d} u_{b}{ }^{e \mu} f_{-d}{ }^{f \nu \lambda} \gamma_{5} \gamma_{\nu} D_{\lambda} T_{A, n, c e f \mu}$ & 14 & 33 & $\epsilon^{a b c} \bar{B}_{a}{ }^{d} u^{e f \mu} \chi_{+b e} T_{A, n, c d f \mu}$ & & 65 \\
\hline$\epsilon^{a b c} \bar{B}_{a}{ }^{d} u_{b}{ }^{e \mu} f_{-e}{ }^{f \nu \lambda} \gamma_{5} \gamma_{\mu} D_{\nu} T_{A, n, c d f \lambda}$ & & 34 & $\epsilon^{a b c} \bar{B}_{a}{ }^{d} u_{b}{ }^{e \mu} \chi_{+, s} T_{A, n, c d e \mu}$ & 32 & 66 \\
\hline$\epsilon^{a b c} \bar{B}_{a}{ }^{d} u_{b}{ }^{e \mu} f_{-e}{ }^{f \nu \lambda} \gamma_{5} \gamma_{\nu} D_{\mu} T_{A, n, c d f \lambda}$ & & 35 & $i \epsilon^{a b c} \bar{B}_{a}{ }^{d} \nabla^{\mu} \chi_{-b}{ }^{e} T_{A, n, c d e \mu}$ & 33 & 67 \\
\hline
\end{tabular}

TABLE VII. Terms in the $\mathcal{O}\left(p^{4}\right)$ meson-octet-decuplet and $\pi N \Delta$ chiral Lagrangians, where $P_{n}^{\left(N_{f}, 4\right)}$ is defined in Eqs. (64) and (66). For the $S U(2)$ case, the form needs to be changed; see the sentences around Eq. (67).

\begin{tabular}{|c|c|c|c|c|c|}
\hline$\overline{P_{n}^{\left(N_{f}, 4\right)}}$ & $S U(2)$ & $S U(3)$ & $P_{n}^{\left(N_{f}, 4\right)}$ & $S U(2)$ & $S U(3)$ \\
\hline $\bar{\epsilon}^{a b c} \bar{B}_{a}{ }^{d} u_{b}{ }^{e \mu} u_{c}{ }^{f}{ }_{\mu} u_{d}{ }^{g \nu} u_{e}{ }^{h \lambda} \gamma_{5} \gamma_{\nu} T_{A, n, f g h \lambda}$ & 1 & 1 & $\epsilon^{a b c} \bar{B}_{a}{ }^{d} u_{b}{ }^{e \mu} \nabla^{\nu} f_{-c}{ }^{f}{ }_{\mu}^{\lambda} \gamma_{5} \gamma_{\nu} T_{A, n, \text { def } \lambda}$ & 78 & 322 \\
\hline$\epsilon^{a b c} \bar{B}_{a}{ }^{d} u_{b}{ }^{e \mu} u_{c}{ }^{f}{ }_{\mu} u_{d}{ }^{g \nu} u_{e}{ }^{h \lambda} \gamma_{5} \gamma_{\lambda} T_{A, n, f g h \nu}$ & 2 & 2 & $\epsilon^{a b c} \bar{B}_{a}{ }^{d} u_{b}{ }^{e \mu} \nabla^{\nu} f_{-c}{ }^{f}{ }^{\lambda} \gamma_{5} \gamma_{\mu} T_{A, n, \text { def } \lambda}$ & 79 & 323 \\
\hline$\epsilon^{a b c} \bar{B}_{a}{ }^{d} u_{b}{ }^{e \mu} u_{c}{ }^{f}{ }_{\mu} u_{e}{ }^{g \nu} u_{f}{ }^{h \lambda} \gamma_{5} \gamma_{\nu} T_{A, n, d g h \lambda}$ & & 3 & $\epsilon^{a b c} \bar{B}_{a}^{d}{ }^{d} u_{b}{ }^{e \mu} \nabla^{\nu} f_{-c}{ }^{f}{ }^{\lambda} \gamma_{5} \gamma_{\lambda} T_{A, n, \text { def } \mu}$ & 80 & 324 \\
\hline$\epsilon^{a b c} \bar{B}_{a}{ }^{d} u_{b}{ }^{e \mu} u_{c}{ }^{f}{ }_{\mu} u_{e}{ }^{g \nu} u_{g}{ }^{h \lambda} \gamma_{5} \gamma_{\nu} T_{A, n, d f h \lambda}$ & & 4 & $\epsilon^{a b c} \bar{B}_{a}{ }^{d} u_{b}{ }^{e \mu} \nabla_{\mu} f_{-d}{ }^{f \nu \lambda} \gamma_{5} \gamma_{\nu} T_{A, n, c e f \lambda}$ & 81 & 325 \\
\hline$\epsilon^{a b c} \bar{B}_{a}{ }^{d} u_{b}{ }^{e \mu} u_{c}{ }^{f}{ }_{\mu} u_{e} e^{g \nu} u_{g}{ }^{h \lambda} \gamma_{5} \gamma_{\lambda} T_{A, n, d f h \nu}$ & & 5 & $\epsilon^{a b c} \bar{B}_{a}{ }^{d} u_{b}{ }^{e \mu} \nabla^{\nu} f_{-d}{ }^{f}{ }_{\mu}^{\lambda} \gamma_{5} \gamma_{\nu} T_{A, n, c e f \lambda}$ & 82 & 326 \\
\hline$\epsilon^{a b c} \bar{B}_{a}{ }^{d} u_{b}{ }^{e \mu} u_{c}{ }^{f \nu} u_{d}{ }_{\mu} u_{e}{ }^{h \lambda} \gamma_{5} \gamma_{\nu} T_{A, n, f g h \lambda}$ & 3 & 6 & $\epsilon^{a b c} \bar{B}_{a}^{d}{ }^{d} u_{b}{ }^{e \mu} \nabla^{\nu} f_{-d} f_{\nu}{ }^{\lambda} \gamma_{5} \gamma_{\mu} T_{A, n, c e f \lambda}$ & 83 & 327 \\
\hline$\epsilon^{a b c} \bar{B}_{a}^{d} u_{b}{ }^{e \mu} u_{c}{ }^{f \nu} u_{d}{ }^{g} u_{e} u_{e}{ }^{h \lambda} \gamma_{5} \gamma_{\lambda} T_{A, n, f g h \nu}$ & & 7 & $\epsilon^{a b c} \bar{B}_{a}^{d} u_{b}{ }^{e \mu} \nabla^{\nu} f_{-d^{f} \nu^{\lambda} \gamma_{5} \gamma_{\lambda} T_{A, n, \text { cef } \mu}}$ & 84 & 328 \\
\hline$\epsilon^{a b c} \bar{B}_{a}{ }^{d} u_{b}{ }^{e \mu} u_{c}{ }^{f \nu} u_{d}{ }^{g}{ }_{\mu} u_{f}{ }^{h \lambda} \gamma_{5} \gamma_{\nu} T_{A, n, e g h \lambda}$ & 4 & 8 & $\epsilon^{a b c} \bar{B}_{a}{ }^{d} u_{b}{ }^{e \mu} \nabla_{\mu} f_{-e}{ }^{f \nu \lambda} \gamma_{5} \gamma_{\nu} T_{A, n, c d f \lambda}$ & & 329 \\
\hline$\epsilon^{a b c} \bar{B}_{a}^{d}{ }^{d} u_{b}{ }^{e \mu} u_{c}{ }^{f \nu} u_{d}{ }^{g}{ }_{\mu} u_{f}{ }^{h \lambda} \gamma_{5} \gamma_{\lambda} T_{A, n, e g h \nu}$ & 5 & 9 & $\epsilon^{a b c} \bar{B}_{a}{ }^{d} u_{b}{ }^{e \mu} \nabla^{\nu} f_{-e}{ }^{f}{ }_{\mu}^{\lambda} \gamma_{5} \gamma_{\nu} T_{A, n, c d f \lambda}$ & & 330 \\
\hline$\epsilon^{a b c} \bar{B}_{a}{ }^{d} u_{b}{ }^{e \mu} u_{c}{ }^{f \nu} u_{d}{ }^{g \lambda} u_{e}{ }_{\mu}{ }_{\mu} \gamma_{5} \gamma_{\nu} T_{A, n, f g h \lambda}$ & & 10 & $\epsilon^{a b c} \bar{B}_{a}{ }^{d} u_{b}{ }^{e \mu} \nabla^{\nu} f_{-e}{ }^{f}{ }_{\nu}{ }^{\lambda} \gamma_{5} \gamma_{\mu} T_{A, n, c d f \lambda}$ & & 331 \\
\hline$\epsilon^{a b c} \bar{B}_{a}{ }^{d} u_{b}{ }^{e \mu} u_{c}{ }^{f \nu} u_{d}{ }^{g \lambda} u_{e}{ }^{h}{ }_{\mu} \gamma_{5} \gamma_{\lambda} T_{A, n, f g h \nu}$ & & 11 & $\epsilon^{a b c} \bar{B}_{a}{ }^{d} u_{b}{ }^{e \mu} \nabla^{\nu} f_{-e}{ }^{f}{ }_{\nu}{ }^{\lambda} \gamma_{5} \gamma_{\lambda} T_{A, n, c d f \mu}$ & & 332 \\
\hline$\epsilon^{a b c} \bar{B}_{a}{ }^{d} u_{b}{ }^{e \mu} u_{c}{ }^{f \nu} u_{d}{ }^{g \lambda} u_{e}{ }^{h}{ }_{\nu} \gamma_{5} \gamma_{\mu} T_{A, n, f g h \lambda}$ & 6 & 12 & $\epsilon^{a b c} \bar{B}_{a}{ }^{d} u^{e f \mu} \nabla_{\mu} f_{-b e}{ }^{\nu \lambda} \gamma_{5} \gamma_{\nu} T_{A, n, c d f \lambda}$ & & 333 \\
\hline$\epsilon^{a b c} \bar{B}_{a}{ }^{d} u_{b}{ }^{e \mu} u_{c}{ }^{f \nu} u_{d}{ }^{g \lambda} u_{e}{ }^{h}{ }_{\nu} \gamma_{5} \gamma_{\lambda} T_{A, n, f g h \mu}$ & 7 & 13 & $\epsilon^{a b c} \bar{B}_{a}{ }^{d} u^{e f \mu} \nabla^{\nu} f_{-b e \mu}{ }^{\lambda} \gamma_{5} \gamma_{\nu} T_{A, n, c d f \lambda}$ & & 334 \\
\hline$\epsilon^{a b c} \bar{B}_{a}^{d} u_{b}{ }^{e \mu} u_{c}{ }^{f \nu} u_{d}{ }^{g \lambda} u_{e}{ }_{\lambda}{ }_{\lambda} \gamma_{5} \gamma_{\mu} T_{A, n, f g h \nu}$ & & 14 & $\epsilon^{a b c} \bar{B}_{a}^{d}{ }^{d} u^{e f \mu} \nabla^{\nu} f_{-b e \nu}{ }^{\lambda} \gamma_{5} \gamma_{\mu} T_{A, n, c d f \lambda}$ & & 335 \\
\hline$\epsilon^{a b c} \bar{B}_{a}{ }^{d} u_{b}{ }^{e \mu} u_{c}{ }^{f \nu} u_{d}{ }^{g \lambda} u_{e}{ }^{h}{ }_{\lambda} \gamma_{5} \gamma_{\nu} T_{A, n, f g h \mu}$ & & 15 & $\epsilon^{a b c} \bar{B}_{a}{ }^{d} u^{e f \mu} \nabla^{\nu} f_{-b e \nu}{ }^{\lambda} \gamma_{5} \gamma_{\lambda} T_{A, n, c d f \mu}$ & & 336 \\
\hline$\epsilon^{a b c} \bar{B}_{a}^{d}{ }^{d} u_{b}{ }^{e \mu} u_{c}{ }^{f \nu} u_{e}{ }^{g} u_{f}{ }_{f}{ }^{h \lambda} \gamma_{5} \gamma_{\nu} T_{A, n, d g h \lambda}$ & & 16 & $\epsilon^{a b c} \bar{B}_{a}{ }^{d} u_{b}{ }^{e \mu} \nabla_{\mu} h_{c}{ }^{f \nu \lambda} \gamma_{5} \gamma_{\nu} T_{A, n, \text { def } \lambda}$ & 85 & 337 \\
\hline$\epsilon^{a b c} \bar{B}_{a}^{d} u_{b}{ }^{e \mu} u_{c}{ }^{f \nu} u_{e}{ }^{g}{ }_{\mu} u_{f}{ }^{h \lambda} \gamma_{5} \gamma_{\lambda} T_{A, n, d g h \nu}$ & & 17 & $\epsilon^{a b c} \bar{B}_{a}^{d} u_{b}^{e \mu} \nabla_{\mu} h_{d}{ }^{f \nu \lambda} \gamma_{5} \gamma_{\nu} T_{A, n, c e f \lambda}$ & 86 & 338 \\
\hline$\epsilon^{a b c} \bar{B}_{a}{ }^{d} u_{b}{ }^{e \mu} u_{c}{ }^{f \nu} u_{e}{ }^{g}{ }_{\mu} u_{g}{ }^{h \lambda} \gamma_{5} \gamma_{\nu} T_{A, n, d f h \lambda}$ & & 18 & $\epsilon^{a b c} \bar{B}_{a}{ }^{d} u_{b}{ }^{e \mu} \nabla_{\mu} h_{e}{ }^{f \nu \lambda} \gamma_{5} \gamma_{\nu} T_{A, n, c d f \lambda}$ & & 339 \\
\hline$\epsilon^{a b c} \bar{B}_{a}{ }^{d} u_{b}{ }^{e \mu} u_{c}{ }^{f \nu} u_{e}{ }^{g}{ }_{\mu} u_{g}{ }^{h \lambda} \gamma_{5} \gamma_{\lambda} T_{A, n, d f h \nu}$ & & 19 & $\epsilon^{a b c} \bar{B}_{a}^{d} u^{e f \mu} \nabla_{\mu} h_{b e}{ }^{\nu \lambda} \gamma_{5} \gamma_{\nu} T_{A, n, c d f \lambda}$ & & 340 \\
\hline
\end{tabular}


TABLE VII. (Continued)

\begin{tabular}{|c|c|c|c|c|c|}
\hline$P_{n}^{\left(N_{f}, 4\right)}$ & $S U(2)$ & $S U(3)$ & $P_{n}^{\left(N_{f}, 4\right)}$ & $S U(2)$ & $S U(3)$ \\
\hline$\epsilon^{a b c} \bar{B}_{a}{ }^{d} u_{b}{ }^{e \mu} u_{c}{ }^{f \nu} u_{e}{ }_{\nu}{ }_{\nu} u_{f}{ }^{h \lambda} \gamma_{5} \gamma_{\mu} T_{A, n, d g h \lambda}$ & & 20 & $\varepsilon^{\mu \nu \lambda \rho} \epsilon^{a b c} \bar{B}_{a}{ }^{d} f_{-b}{ }^{e}{ }_{\mu \nu} f_{-c}{ }^{f}{ }_{\lambda}{ }^{\sigma} D_{\rho} T_{A, n, \operatorname{def} \sigma}$ & 87 & 341 \\
\hline$\epsilon^{a b c} \bar{B}_{a}{ }^{d} u_{b}{ }^{e \mu} u_{c}{ }^{f \nu} u_{e}{ }_{\nu}{ }_{\nu} u_{f}{ }^{h \lambda} \gamma_{5} \gamma_{\lambda} T_{A, n, d g h \mu}$ & & 21 & $\varepsilon^{\mu \nu \lambda \rho} \epsilon^{a b c} \bar{B}_{a}{ }^{d} f_{-b}{ }^{e} \mu \nu f_{-e^{f}{ }_{\lambda}{ }^{\sigma} D_{\rho} T_{A, n, c d f \sigma}}$ & & 342 \\
\hline$\epsilon^{a b c} \bar{B}_{a}{ }^{d} u_{b}{ }^{e \mu} u_{c}{ }^{f \nu} u_{e}{ }_{\nu}{ } u_{g}{ }^{h \lambda} \gamma_{5} \gamma_{\mu} T_{A, n, d f h \lambda}$ & & 22 & $\varepsilon^{\mu \nu \lambda \rho} \epsilon^{a b c} \bar{B}_{a}{ }^{d} h_{b}{ }^{e}{ }_{\mu}{ }^{\sigma} f_{-c}{ }_{\nu \lambda}^{f} D_{\rho} T_{A, n, \operatorname{def} \sigma}$ & 88 & 343 \\
\hline$\epsilon^{a b c} \bar{B}_{a}{ }^{d} u_{b}{ }^{e \mu} u_{c}{ }^{f \nu} u_{e}{ }_{\nu}{ }_{\nu} u_{g}{ }^{h \lambda} \gamma_{5} \gamma_{\lambda} T_{A, n, d f h \mu}$ & & 23 & $\varepsilon^{\mu \nu \lambda \rho} \epsilon^{a b c} \bar{B}_{a}{ }^{d} h_{b}{ }^{e}{ }_{\mu}^{\sigma} f_{-d}{ }^{f}{ }_{\nu \lambda} D_{\rho} T_{A, n, c e f \sigma}$ & 89 & 344 \\
\hline$\epsilon^{a b c} \bar{B}_{a}{ }^{d} u_{b}{ }^{e \mu} u_{c}{ }^{f \nu} u_{e}{ }^{g \lambda} u_{f}^{h}{ }_{\lambda} \gamma_{5} \gamma_{\mu} T_{A, n, d g h \nu}$ & & 24 & $\varepsilon^{\mu \nu \lambda \rho} \epsilon^{a b c} \bar{B}_{a}{ }^{d} h_{b}{ }^{e}{ }_{\mu}^{\sigma} f_{-e^{f}{ }_{\nu \lambda} D_{\rho} T_{A, n, c d f \sigma}}$ & & 345 \\
\hline$\epsilon^{a b c} \bar{B}_{a}{ }^{d} u_{b}{ }^{e \mu} u_{c}{ }^{f \nu} u_{e}{ }^{g \lambda} u_{g}{ }^{h}{ }_{\mu} \gamma_{5} \gamma_{\nu} T_{A, n, d f h \lambda}$ & & 25 & $\varepsilon^{\mu \nu \lambda \rho} \epsilon^{a b c} \bar{B}_{a}{ }^{d} h^{e f}{ }_{\mu} \sigma f_{-b e \nu \lambda} D_{\rho} T_{A, n, c d f \sigma}$ & & 346 \\
\hline$\epsilon^{a b c} \bar{B}_{a}{ }^{d} u_{b}{ }^{e \mu} u_{c}{ }^{f \nu} u_{e}{ }^{g \lambda} u_{g_{\mu}}{ }^{\mu} \gamma_{5} \gamma_{\lambda} T_{A, n, d f h \nu}$ & & 26 & $i \epsilon^{a b c} \bar{B}_{a}{ }^{d} f_{+b}{ }^{e \mu \nu} u_{c}{ }^{f}{ }_{\mu} u_{d}{ }^{g \lambda} \gamma_{5} \gamma_{\nu} T_{A, n, e f g \lambda}$ & 90 & 347 \\
\hline$\epsilon^{a b c} \bar{B}_{a}{ }^{d} u_{b}{ }^{e \mu} u_{c}{ }^{f \nu} u_{e}{ }^{g \lambda} u_{g}{ }^{h}{ }_{\nu} \gamma_{5} \gamma_{\mu} T_{A, n, d f h \lambda}$ & & 27 & $i \epsilon^{a b c} \bar{B}_{a}{ }^{d} f_{+b}{ }^{e \mu \nu} u_{c}{ }^{f}{ }_{\mu} u_{d}{ }^{g \lambda} \gamma_{5} \gamma_{\lambda} T_{A, n, e f g \nu}$ & 91 & 348 \\
\hline$\epsilon^{a b c} \bar{B}_{a}{ }^{d} u_{b}{ }^{e \mu} u_{c}{ }^{f \nu} u_{e}{ }^{g \lambda} u_{g}{ }^{h}{ }_{\nu} \gamma_{5} \gamma_{\lambda} T_{A, n, d f h \mu}$ & & 28 & $i \epsilon^{a b c} \bar{B}_{a}{ }^{d} f_{+b}{ }^{e \mu \nu} u_{c}{ }^{f}{ }_{\mu} u_{e}^{g \lambda} \gamma_{5} \gamma_{\nu} T_{A, n, d f g \lambda}$ & 92 & 349 \\
\hline$\epsilon^{a b c} \bar{B}_{a}{ }^{d} u_{b}{ }^{e \mu} u_{c}{ }^{f \nu} u_{e}{ }^{g \lambda} u_{g}{ }_{\lambda}{ }_{\lambda} \gamma_{5} \gamma_{\mu} T_{A, n, d f h \nu}$ & & 29 & $i \epsilon^{a b c} \bar{B}_{a}^{d} f_{+b}{ }^{e \mu \nu} u_{c}{ }^{f}{ }_{\mu} u_{e}{ }^{g \lambda} \gamma_{5} \gamma_{\lambda} T_{A, n, d f g \nu}$ & 93 & 350 \\
\hline$\epsilon^{a b c} \bar{B}_{a}{ }^{d} u_{b}{ }^{e \mu} u_{c}{ }^{f \nu} u_{e}{ }^{g \lambda} u_{g}{ }^{h}{ }_{\lambda} \gamma_{5} \gamma_{\nu} T_{A, n, d f h \mu}$ & & 30 & $i \epsilon^{a b c} \bar{B}_{a}^{d} f_{+b}{ }^{e \mu \nu} u_{c}{ }^{f}{ }_{\mu} u_{f}{ }^{g \lambda} \gamma_{5} \gamma_{\nu} T_{A, n, \operatorname{deg} \lambda}$ & 94 & 351 \\
\hline$\epsilon^{a b c} \bar{B}_{a}{ }^{d} u_{b}{ }^{e \mu} u_{d}{ }_{\mu}^{f} u_{e}{ }^{g \nu} u_{f}{ }^{h \lambda} \gamma_{5} \gamma_{\nu} T_{A, n, c g h \lambda}$ & & 31 & $i \epsilon^{a b c} \bar{B}_{a}{ }^{d} f_{+b}{ }^{e \mu \nu} u_{c}{ }^{f}{ }_{\mu} u_{f}{ }^{g \lambda} \gamma_{5} \gamma_{\lambda} T_{A, n, \operatorname{deg} \nu}$ & 95 & 352 \\
\hline$\epsilon^{a b c} \bar{B}_{a}{ }^{d} u_{b}{ }^{e \mu} u_{d}{ }_{\mu}^{f} u_{e}{ }^{g \nu} u_{g}{ }^{h \lambda} \gamma_{5} \gamma_{\nu} T_{A, n, c f h \lambda}$ & & 32 & $i \epsilon^{a b c} \bar{B}_{a}^{d} f_{+b}{ }^{e \mu \nu} u_{c}{ }^{f \lambda} u_{d^{g}}^{g} \gamma_{5} \gamma_{\nu} T_{A, n, e f g \lambda}$ & 96 & 353 \\
\hline$\epsilon^{a b c} \bar{B}_{a}{ }^{d} u_{b}{ }^{e \mu} u_{d}{ }_{\mu}^{f} u_{e}{ }^{g \nu} u_{g}{ }^{h \lambda} \gamma_{5} \gamma_{\lambda} T_{A, n, c f h \nu}$ & & 33 & $i \epsilon^{a b c} \bar{B}_{a}^{d} f_{+b}{ }^{e \mu \nu} u_{c}{ }^{f \lambda} u_{d^{g}}{ }_{\mu} \gamma_{5} \gamma_{\lambda} T_{A, n, \text { ef } g \nu}$ & 97 & 354 \\
\hline$\epsilon^{a b c} \bar{B}_{a}{ }^{d} u_{b}{ }^{e \mu} u_{d}{ }^{f}{ }_{\mu} u^{g h \nu} u_{h g}{ }^{\lambda} \gamma_{5} \gamma_{\nu} T_{A, n, c e f \lambda}$ & & 34 & $i \epsilon^{a b c} \bar{B}_{a}^{d} f_{+b}{ }^{e \mu \nu} u_{c}{ }^{f \lambda} u_{d}{ }_{\lambda}^{g} \gamma_{5} \gamma_{\mu} T_{A, n, e f g \nu}$ & 98 & 355 \\
\hline$\epsilon^{a b c} \bar{B}_{a}{ }^{d} u_{b}{ }^{e \mu} u_{d}{ }^{f \nu} u_{e}{ }_{\mu}^{g} u_{f}{ }^{h \lambda} \gamma_{5} \gamma_{\nu} T_{A, n, c g h \lambda}$ & & 35 & $i \epsilon^{a b c} \bar{B}_{a}{ }^{d} f_{+b}{ }^{e \mu \nu} u_{c}{ }^{f \lambda} u_{e}{ }_{\mu}^{g} \gamma_{5} \gamma_{\nu} T_{A, n, d f g \lambda}$ & & 356 \\
\hline$\epsilon^{a b c} \bar{B}_{a}{ }^{d} u_{b}{ }^{e \mu} u_{d}{ }^{f \nu} u_{e}{ }_{\mu}{ }_{\mu} u_{f}{ }^{h \lambda} \gamma_{5} \gamma_{\lambda} T_{A, n, c g h \nu}$ & & 36 & $i \epsilon^{a b c} \bar{B}_{a}^{d} f_{+b}{ }^{e \mu \nu} u_{c}{ }^{f \lambda} u_{e}^{g}{ }_{\mu} \gamma_{5} \gamma_{\lambda} T_{A, n, d f g \nu}$ & & 357 \\
\hline$\epsilon^{a b c} \bar{B}_{a}{ }^{d} u_{b}{ }^{e \mu} u_{d}{ }^{f \nu} u_{e}{ }_{\mu}{ }_{\mu} u_{g}{ }^{h \lambda} \gamma_{5} \gamma_{\nu} T_{A, n, c f h \lambda}$ & & 37 & $i \epsilon^{a b c} \bar{B}_{a}^{d} f_{+b}{ }^{e \mu \nu} u_{c}{ }^{f \lambda} u_{e}^{g}{ }_{\lambda} \gamma_{5} \gamma_{\mu} T_{A, n, d f g \nu}$ & 99 & 358 \\
\hline$\epsilon^{a b c} \bar{B}_{a}{ }^{d} u_{b}{ }^{e \mu} u_{d}{ }^{f \nu} u_{e}{ }_{\nu}{ }_{\nu} u_{f}{ }^{h \lambda} \gamma_{5} \gamma_{\mu} T_{A, n, c g h \lambda}$ & & 38 & $i \epsilon^{a b c} \bar{B}_{a}^{d} f_{+b}{ }^{e \mu \nu} u_{c}{ }^{f \lambda} u_{f}^{g}{ }_{\mu} \gamma_{5} \gamma_{\nu} T_{A, n, \operatorname{deg} \lambda}$ & 100 & 359 \\
\hline$\epsilon^{a b c} \bar{B}_{a}{ }^{d} u_{b}{ }^{e \mu} u_{d}{ }^{f \nu} u_{e}{ }_{\nu}^{g} u_{f}{ }^{h \lambda} \gamma_{5} \gamma_{\lambda} T_{A, n, c g h \mu}$ & & 39 & $i \epsilon^{a b c} \bar{B}_{a}{ }^{d} f_{+b}{ }^{e \mu \nu} u_{c}{ }^{f \lambda} u_{f}^{g}{ }_{\mu}^{\mu} \gamma_{5} \gamma_{\lambda} T_{A, n, \operatorname{deg} \nu}$ & 101 & 360 \\
\hline$\epsilon^{a b c} \bar{B}_{a}{ }^{d} u_{b}{ }^{e \mu} u_{d}{ }^{f \nu} u_{e}{ }_{\nu}{ }_{\nu} u_{g}{ }^{h \lambda} \gamma_{5} \gamma_{\mu} T_{A, n, c f h \lambda}$ & & 40 & $i \epsilon^{a b c} \bar{B}_{a}{ }^{d} f_{+b}{ }^{e \mu \nu} u_{c}{ }^{f \lambda} u_{f}{ }_{\lambda}{ }_{\lambda} \gamma_{5} \gamma_{\mu} T_{A, n, \operatorname{deg} \nu}$ & 102 & 361 \\
\hline$\epsilon^{a b c} \bar{B}_{a}{ }^{d} u_{b}{ }^{e \mu} u_{d}{ }^{f \nu} u_{e}{ }_{\nu}{ }_{\nu} u_{g}{ }^{h \lambda} \gamma_{5} \gamma_{\lambda} T_{A, n, c f h \mu}$ & & 41 & $i \epsilon^{a b c} \bar{B}_{a}^{d} f_{+b}{ }^{e \mu \nu} u_{d}{ }_{\mu}^{f} u_{e}{ }^{g \lambda} \gamma_{5} \gamma_{\nu} T_{A, n, c f g \lambda}$ & & 362 \\
\hline$\epsilon^{a b c} \bar{B}_{a}{ }^{d} u_{b}{ }^{e \mu} u_{d}{ }^{f \nu} u_{e}{ }^{g \lambda} u_{f}{ }_{\lambda}^{h} \gamma_{5} \gamma_{\mu} T_{A, n, c g h \nu}$ & & 42 & $i \epsilon^{a b c} \bar{B}_{a}{ }^{d} f_{+b}{ }^{e \mu \nu} u_{d}{ }_{\mu}^{f} u_{e}{ }^{g \lambda} \gamma_{5} \gamma_{\lambda} T_{A, n, c f g \nu}$ & & 363 \\
\hline$\epsilon^{a b c} \bar{B}_{a}{ }^{d} u_{b}{ }^{e \mu} u_{d}{ }^{f \nu} u_{e}{ }^{g \lambda} u_{g}{ }^{h}{ }_{\nu} \gamma_{5} \gamma_{\mu} T_{A, n, c f h \lambda}$ & & 43 & $i \epsilon^{a b c} \bar{B}_{a}^{d} f_{+b}{ }^{e \mu \nu} u_{d}{ }_{\mu}^{f} u_{f}{ }^{g \lambda} \gamma_{5} \gamma_{\nu} T_{A, n, c e g \lambda}$ & & 364 \\
\hline$\epsilon^{a b c} \bar{B}_{a}{ }^{d} u_{b}{ }^{e \mu} u_{e}{ }_{\mu}^{f} u_{f}^{g \nu} u_{g}^{h \lambda} \gamma_{5} \gamma_{\nu} T_{A, n, c d h \lambda}$ & & 44 & $i \epsilon^{a b c} \bar{B}_{a}{ }^{d} f_{+b}{ }^{e \mu \nu} u_{d}{ }_{\mu}^{f} u_{f}{ }^{g \lambda} \gamma_{5} \gamma_{\lambda} T_{A, n, c e g \nu}$ & & 365 \\
\hline$\epsilon^{a b c} \bar{B}_{a}{ }^{d} u_{b}{ }^{e \mu} u_{e}{ }_{\mu}{ }_{\mu} u_{f}^{g \nu} u_{g}{ }^{h \lambda} \gamma_{5} \gamma_{\lambda} T_{A, n, c d h \nu}$ & & 45 & $i \epsilon^{a b c} \bar{B}_{a}^{d} f_{+b}{ }^{e \mu \nu} u_{d}{ }^{f \lambda} u_{e}{ }_{\mu} \gamma_{5} \gamma_{\nu} T_{A, n, c f g \lambda}$ & & 366 \\
\hline$\epsilon^{a b c} \bar{B}_{a}{ }^{d} u_{b}{ }^{e \mu} u_{e}{ }^{f}{ }_{\mu} u^{g h \nu} u_{h g}{ }^{\lambda} \gamma_{5} \gamma_{\nu} T_{A, n, c d f \lambda}$ & & 46 & $i \epsilon^{a b c} \bar{B}_{a}^{d} f_{+b}{ }^{e \mu \nu} u_{d}^{f \lambda} u_{e}^{g}{ }_{\mu} \gamma_{5} \gamma_{\lambda} T_{A, n, c f g \nu}$ & & 367 \\
\hline$\epsilon^{a b c} \bar{B}_{a}{ }^{d} u_{b}{ }^{e \mu} u_{e}{ }^{f \nu} u_{f}{ }_{\mu}^{g} u_{g}{ }^{h \lambda} \gamma_{5} \gamma_{\nu} T_{A, n, c d h \lambda}$ & & 47 & $i \epsilon^{a b c} \bar{B}_{a}^{d} f_{+b}{ }^{e \mu \nu} u_{d}^{f \lambda} u_{e}{ }_{\lambda} \gamma_{5} \gamma_{\mu} T_{A, n, c f g \nu}$ & & 368 \\
\hline$\epsilon^{a b c} \bar{B}_{a}{ }^{d} u_{b}{ }^{e \mu} u_{e}{ }^{f \nu} u_{f}{ }_{\mu}^{g} u_{g}{ }^{h \lambda} \gamma_{5} \gamma_{\lambda} T_{A, n, c d h \nu}$ & & 48 & $i \epsilon^{a b c} \bar{B}_{a}^{d} f_{+b}{ }^{e \mu \nu} u_{d}{ }^{f \lambda} u_{f}^{g}{ }_{\mu} \gamma_{5} \gamma_{\nu} T_{A, n, c e g \lambda}$ & & 369 \\
\hline$\epsilon^{a b c} \bar{B}_{a}{ }^{d} u_{b}{ }^{e \mu} u_{e}{ }^{f \nu} u_{f}{ }^{g \lambda} u_{g}{ }^{h}{ }_{\mu} \gamma_{5} \gamma_{\nu} T_{A, n, c d h \lambda}$ & & 49 & $i \epsilon^{a b c} \bar{B}_{a}^{d} f_{+b}{ }^{e \mu \nu} u_{d}{ }^{f \lambda} u_{f}{ }_{\mu}{ }_{\mu} \gamma_{5} \gamma_{\lambda} T_{A, n, c e g \nu}$ & & 370 \\
\hline$\epsilon^{a b c} \bar{B}_{a}{ }^{d} u_{b}{ }^{e \mu} u_{e}{ }^{f \nu} u_{f}{ }^{g \lambda} u_{g}{ }^{h}{ }_{\nu} \gamma_{5} \gamma_{\mu} T_{A, n, c d h \lambda}$ & & 50 & $i \epsilon^{a b c} \bar{B}_{a}{ }^{d} f_{+b}{ }^{e \mu \nu} u_{d}{ }^{f \lambda} u_{f}{ }_{\lambda}{ }_{\lambda} \gamma_{5} \gamma_{\mu} T_{A, n, c e g \nu}$ & & 371 \\
\hline$\epsilon^{a b c} \bar{B}_{a}{ }^{d} u_{b}{ }^{e \mu} u_{e}{ }^{f \nu} u_{f}{ }^{g \lambda} u_{g}{ }^{h}{ }_{\nu} \gamma_{5} \gamma_{\lambda} T_{A, n, c d h \mu}$ & & 51 & $i \epsilon^{a b c} \bar{B}_{a}^{d} f_{+b}{ }^{e \mu \nu} u_{e}{ }_{\mu}^{f} u_{f}{ }^{g \lambda} \gamma_{5} \gamma_{\nu} T_{A, n, c d g \lambda}$ & & 372 \\
\hline$\epsilon^{a b c} \bar{B}_{a}{ }^{d} u_{b}{ }^{e \mu} u_{c}{ }^{f \nu} u_{d}{ }^{g \lambda} u_{e}{ }^{h \rho} \gamma_{5} \gamma_{\mu} D_{\nu \lambda} T_{A, n, f g h \rho}$ & 8 & 52 & $i \epsilon^{a b c} \bar{B}_{a}^{d} f_{+b}{ }^{e \mu \nu} u_{e}{ }_{\mu}^{f} u_{f}{ }^{g \lambda} \gamma_{5} \gamma_{\lambda} T_{A, n, c d g \nu}$ & & 373 \\
\hline$\epsilon^{a b c} \bar{B}_{a}{ }^{d} u_{b}{ }^{e \mu} u_{c}{ }^{f \nu} u_{d}{ }^{g \lambda} u_{e}{ }^{h \rho} \gamma_{5} \gamma_{\mu} D_{\nu \rho} T_{A, n, f g h \lambda}$ & 9 & 53 & $i \epsilon^{a b c} \bar{B}_{a}^{d} f_{+b}{ }^{e \mu \nu} u_{e}{ }^{f \lambda} u_{f}^{g}{ }_{\mu} \gamma_{5} \gamma_{\nu} T_{A, n, c d g \lambda}$ & & 374 \\
\hline$\epsilon^{a b c} \bar{B}_{a}{ }^{d} u_{b}{ }^{e \mu} u_{c}{ }^{f \nu} u_{d}{ }^{g \lambda} u_{e}{ }^{h \rho} \gamma_{5} \gamma_{\mu} D_{\lambda \rho} T_{A, n, f g h \nu}$ & & 54 & $i \epsilon^{a b c} \bar{B}_{a}^{d} f_{+b}{ }^{e \mu \nu} u_{e}{ }^{f \lambda} u_{f}^{g}{ }_{\mu} \gamma_{5} \gamma_{\lambda} T_{A, n, c d g \nu}$ & & 375 \\
\hline$\epsilon^{a b c} \bar{B}_{a}{ }^{d} u_{b}{ }^{e \mu} u_{c}{ }^{f \nu} u_{d}{ }^{g \lambda} u_{e}{ }^{h \rho} \gamma_{5} \gamma_{\nu} D_{\mu \lambda} T_{A, n, f g h \rho}$ & 10 & 55 & $i \epsilon^{a b c} \bar{B}_{a}{ }^{d} f_{+b}{ }^{e \mu \nu} u_{e}{ }^{f \lambda} u_{f}^{g}{ }_{\lambda}^{\mu} \gamma_{5} \gamma_{\mu} T_{A, n, c d g \nu}$ & & 376 \\
\hline$\epsilon^{a b c} \bar{B}_{a}{ }^{d} u_{b}{ }^{e \mu} u_{c}{ }^{f \nu} u_{d}{ }^{g \lambda} u_{e}{ }^{h \rho} \gamma_{5} \gamma_{\nu} D_{\mu \rho} T_{A, n, f g h \lambda}$ & 11 & 56 & $i \epsilon^{a b c} \bar{B}_{a}^{d} f_{+b}{ }^{e \mu \nu} u^{f g}{ }_{\mu} u_{g f}^{\lambda} \gamma_{5} \gamma_{\nu} T_{A, n, c d e \lambda}$ & & 377 \\
\hline$\epsilon^{a b c} \bar{B}_{a}{ }^{d} u_{b}{ }^{e \mu} u_{c}{ }^{f \nu} u_{d}{ }^{g \lambda} u_{e}{ }^{h \rho} \gamma_{5} \gamma_{\nu} D_{\lambda \rho} T_{A, n, f g h \mu}$ & 12 & 57 & $i \epsilon^{a b c} \bar{B}_{a}^{d} f_{+b}{ }^{e \mu \nu} u^{f g}{ }_{\mu} u_{g f}^{\lambda} \gamma_{5} \gamma_{\lambda} T_{A, n, c d e \nu}$ & & 378 \\
\hline$\epsilon^{a b c} \bar{B}_{a}{ }^{d} u_{b}{ }^{e \mu} u_{c}{ }^{f \nu} u_{d}{ }^{g \lambda} u_{e}{ }^{h \rho} \gamma_{5} \gamma_{\lambda} D_{\mu \nu} T_{A, n, f g h \rho}$ & 13 & 58 & $i \epsilon^{a b c} \bar{B}_{a}^{d} f_{+b}{ }^{e \mu \nu} u^{f g \lambda} u_{g f \lambda} \gamma_{5} \gamma_{\mu} T_{A, n, c d e \nu}$ & & 379 \\
\hline$\epsilon^{a b c} \bar{B}_{a}{ }^{d} u_{b}{ }^{e \mu} u_{c}{ }^{f \nu} u_{d}{ }^{g \lambda} u_{e}{ }^{h \rho} \gamma_{5} \gamma_{\lambda} D_{\mu \rho} T_{A, n, f g h \nu}$ & & 59 & $i \epsilon^{a b c} \bar{B}_{a}{ }^{d} f_{+}{ }^{e f \mu \nu} u_{b e \mu} u_{c}{ }^{g \lambda} \gamma_{5} \gamma_{\nu} T_{A, n, d f g \lambda}$ & & 380 \\
\hline$\epsilon^{a b c} \bar{B}_{a}{ }^{d} u_{b}{ }^{e \mu} u_{c}{ }^{f \nu} u_{d}{ }^{g \lambda} u_{e}{ }^{h \rho} \gamma_{5} \gamma_{\lambda} D_{\nu \rho} T_{A, n, f g h \mu}$ & 14 & 60 & $i \epsilon^{a b c} \bar{B}_{a}^{d} f_{+}{ }^{e f \mu \nu} u_{b e \mu} u_{c}{ }^{g \lambda} \gamma_{5} \gamma_{\lambda} T_{A, n, d f g \nu}$ & & 381 \\
\hline$\epsilon^{a b c} \bar{B}_{a}{ }^{d} u_{b}{ }^{e \mu} u_{c}{ }^{f \nu} u_{d}{ }^{g \lambda} u_{e}{ }^{h \rho} \gamma_{5} \gamma_{\rho} D_{\mu \nu} T_{A, n, f g h \lambda}$ & & 61 & $i \epsilon^{a b c} \bar{B}_{a}^{d} f_{+}{ }^{e f \mu \nu} u_{b e \mu} u_{d}^{g \lambda} \gamma_{5} \gamma_{\nu} T_{A, n, c f g \lambda}$ & & 382 \\
\hline$\epsilon^{a b c} \bar{B}_{a}{ }^{d} u_{b}{ }^{e \mu} u_{c}{ }^{f \nu} u_{d}{ }^{g \lambda} u_{e}{ }^{h \rho} \gamma_{5} \gamma_{\rho} D_{\mu \lambda} T_{A, n, f g h \nu}$ & & 62 & $i \epsilon^{a b c} \bar{B}_{a}^{d} f_{+}{ }^{e f \mu \nu} u_{b e \mu} u_{d}^{g \lambda} \gamma_{5} \gamma_{\lambda} T_{A, n, c f g \nu}$ & & 383 \\
\hline$\epsilon^{a b c} \bar{B}_{a}{ }^{d} u_{b}{ }^{e \mu} u_{c}{ }^{f \nu} u_{d}{ }^{g \lambda} u_{e}{ }^{h \rho} \gamma_{5} \gamma_{\rho} D_{\nu \lambda} T_{A, n, f g h \mu}$ & & 63 & $i \epsilon^{a b c} \bar{B}_{a}^{d} f_{+}{ }^{e f \mu \nu} u_{b e \mu} u_{f}^{g \lambda} \gamma_{5} \gamma_{\nu} T_{A, n, c d g \lambda}$ & & 384 \\
\hline$\epsilon^{a b c} \bar{B}_{a}^{d} u_{b}{ }^{e \mu} u_{c}{ }^{f \nu} u_{e}{ }^{g \lambda} u_{f}{ }^{h \rho} \gamma_{5} \gamma_{\mu} D_{\nu \lambda} T_{A, n, d g h \rho}$ & & 64 & $i \epsilon^{a b c} \bar{B}_{a}^{d} f_{+}{ }^{e f \mu \nu} u_{b e \mu} u_{f}^{g \lambda} \gamma_{5} \gamma_{\lambda} T_{A, n, c d g \nu}$ & & 385 \\
\hline$\epsilon^{a b c} \bar{B}_{a}{ }^{d} u_{b}{ }^{e \mu} u_{c}{ }^{f \nu} u_{e}{ }^{g \lambda} u_{f}{ }^{h \rho} \gamma_{5} \gamma_{\mu} D_{\nu \rho} T_{A, n, d g h \lambda}$ & & 65 & $i \epsilon^{a b c} \bar{B}_{a}{ }^{d} f_{+}{ }^{e f \mu \nu} u_{b e}{ }^{\lambda} u_{c}{ }^{g}{ }_{\mu} \gamma_{5} \gamma_{\nu} T_{A, n, d f g \lambda}$ & & 386 \\
\hline$\epsilon^{a b c} \bar{B}_{a}{ }^{d} u_{b}{ }^{e \mu} u_{c}{ }^{f \nu} u_{e}{ }^{g \lambda} u_{f}{ }^{h \rho} \gamma_{5} \gamma_{\mu} D_{\lambda \rho} T_{A, n, d g h \nu}$ & & 66 & $i \epsilon^{a b c} \bar{B}_{a}{ }^{d} f_{+}{ }^{e f \mu \nu} u_{b e}{ }^{\lambda} u_{c}{ }^{g}{ }_{\mu} \gamma_{5} \gamma_{\lambda} T_{A, n, d f g \nu}$ & & 387 \\
\hline
\end{tabular}


TABLE VII. (Continued)

\begin{tabular}{|c|c|c|c|c|c|}
\hline$P_{n}^{\left(N_{f}, 4\right)}$ & $S U(2)$ & $S U(3)$ & $P_{n}^{\left(N_{f}, 4\right)}$ & $S U(2)$ & $S U(3)$ \\
\hline$\epsilon^{a b c} \bar{B}_{a}{ }^{d} u_{b}{ }^{e \mu} u_{c}{ }^{f \nu} u_{e}{ }^{g \lambda} u_{f}{ }^{h \rho} \gamma_{5} \gamma_{\lambda} D_{\mu \nu} T_{A, n, d g h \rho}$ & & 67 & $i \epsilon^{a b c} \bar{B}_{a}{ }^{d} f_{+}{ }^{e f \mu \nu} u_{b e}{ }^{\lambda} u_{c}{ }^{g} \lambda \gamma_{5} \gamma_{\mu} T_{A, n, d f g \nu}$ & & 388 \\
\hline$\epsilon^{a b c} \bar{B}_{a}{ }^{d} u_{b}{ }^{e \mu} u_{c}{ }^{f \nu} u_{e}{ }^{g \lambda} u_{f}{ }^{h \rho} \gamma_{5} \gamma_{\lambda} D_{\mu \rho} T_{A, n, d g h \nu}$ & & 68 & $i \epsilon^{a b c} \bar{B}_{a}^{d} f_{+}{ }^{e f \mu \nu} u_{b e}{ }^{\lambda} u_{d}^{g}{ }_{\mu} \gamma_{5} \gamma_{\nu} T_{A, n, c f g \lambda}$ & & 389 \\
\hline$\epsilon^{a b c} \bar{B}_{a}{ }^{d} u_{b}{ }^{e \mu} u_{c}{ }^{f \nu} u_{e}{ }^{g \lambda} u_{f}{ }^{h \rho} \gamma_{5} \gamma_{\lambda} D_{\nu \rho} T_{A, n, d g h \mu}$ & & 69 & $i \epsilon^{a b c} \bar{B}_{a}{ }^{d} f_{+}{ }^{e f \mu \nu} u_{b e}{ }^{\lambda} u_{d}^{g}{ }_{\mu} \gamma_{5} \gamma_{\lambda} T_{A, n, c f g \nu}$ & & 390 \\
\hline$\epsilon^{a b c} \bar{B}_{a}{ }^{d} u_{b}{ }^{e \mu} u_{c}{ }^{f \nu} u_{e}{ }^{g \lambda} u_{g}{ }^{h \rho} \gamma_{5} \gamma_{\mu} D_{\nu \lambda} T_{A, n, d f h \rho}$ & & 70 & $i \epsilon^{a b c} \bar{B}_{a}{ }^{d} f_{+}{ }^{e f \mu \nu} u_{b e}{ }^{\lambda} u_{d}{ }^{g} \gamma_{5} \gamma_{\mu} T_{A, n, c f g \nu}$ & & 391 \\
\hline$\epsilon^{a b c} \bar{B}_{a}{ }^{d} u_{b}{ }^{e \mu} u_{c}{ }^{f \nu} u_{e}{ }^{g \lambda} u_{g}{ }^{h \rho} \gamma_{5} \gamma_{\mu} D_{\nu \rho} T_{A, n, d f h \lambda}$ & & 71 & $i \epsilon^{a b c} \bar{B}_{a}{ }^{d} f_{+}{ }^{e f \mu \nu} u_{b e^{\lambda} u_{f}^{g}{ }_{\mu} \gamma_{5} \gamma_{\nu} T_{A, n, c d g \lambda}}$ & & 392 \\
\hline$\epsilon^{a b c} \bar{B}_{a}{ }^{d} u_{b}{ }^{e \mu} u_{c}{ }^{f \nu} u_{e}{ }^{g \lambda} u_{g}{ }^{h \rho} \gamma_{5} \gamma_{\mu} D_{\lambda \rho} T_{A, n, d f h \nu}$ & & 72 & $i \epsilon^{a b c} \bar{B}_{a}{ }^{d} f_{+}{ }^{e f \mu \nu} u_{b e^{\lambda} u_{f}{ }^{g}{ }_{\mu} \gamma_{5} \gamma_{\lambda} T_{A, n, c d g \nu}}$ & & 393 \\
\hline$\epsilon^{a b c} \bar{B}_{a}{ }^{d} u_{b}{ }^{e \mu} u_{c}{ }^{f \nu} u_{e}{ }^{g \lambda} u_{g}{ }^{h \rho} \gamma_{5} \gamma_{\nu} D_{\mu \lambda} T_{A, n, d f h \rho}$ & & 73 & $i \epsilon^{a b c} \bar{B}_{a}{ }^{d} f_{+}{ }^{e f \mu \nu} u_{b e}{ }^{\lambda} u_{f}^{g}{ }_{\lambda} \gamma_{5} \gamma_{\mu} T_{A, n, c d g \nu}$ & & 394 \\
\hline$\epsilon^{a b c} \bar{B}_{a}{ }^{d} u_{b}{ }^{e \mu} u_{c}{ }^{f \nu} u_{e}{ }^{g \lambda} u_{g}{ }^{h \rho} \gamma_{5} \gamma_{\nu} D_{\mu \rho} T_{A, n, d f h \lambda}$ & & 74 & $i \epsilon^{a b c} \bar{B}_{a}{ }^{d} f_{+}{ }^{e f \mu \nu} u_{b}{ }_{\mu} u_{g e}{ }^{\lambda} \gamma_{5} \gamma_{\nu} T_{A, n, c d f \lambda}$ & & 395 \\
\hline$\epsilon^{a b c} \bar{B}_{a}{ }^{d} u_{b}{ }^{e \mu} u_{c}{ }^{f \nu} u_{e}{ }^{g \lambda} u_{g}{ }^{h \rho} \gamma_{5} \gamma_{\nu} D_{\lambda \rho} T_{A, n, d f h \mu}$ & & 75 & $i \epsilon^{a b c} \bar{B}_{a}^{d} f_{+}{ }^{e f \mu \nu} u_{b}{ }_{\mu}{ }_{\mu} u_{g e}{ }^{\lambda} \gamma_{5} \gamma_{\lambda} T_{A, n, c d f \nu}$ & & 396 \\
\hline$\epsilon^{a b c} \bar{B}_{a}{ }^{d} u_{b}{ }^{e \mu} u_{c}{ }^{f \nu} u_{e}{ }^{g \lambda} u_{g}{ }^{h \rho} \gamma_{5} \gamma_{\lambda} D_{\mu \nu} T_{A, n, d f h \rho}$ & & 76 & $i \epsilon^{a b c} \bar{B}_{a}^{d} f_{+b}{ }^{e \mu \nu} u_{c}{ }^{f \lambda} u_{d}^{g \rho} \gamma_{5} \gamma_{\mu} D_{\nu \lambda} T_{A, n, e f g \rho}$ & 103 & 397 \\
\hline$\epsilon^{a b c} \bar{B}_{a}{ }^{d} u_{b}{ }^{e \mu} u_{c}{ }^{f \nu} u_{e}{ }^{g \lambda} u_{g}{ }^{h \rho} \gamma_{5} \gamma_{\lambda} D_{\mu \rho} T_{A, n, d f h \nu}$ & & 77 & $i \epsilon^{a b c} \bar{B}_{a}^{d} f_{+b}{ }^{e \mu \nu} u_{c}{ }^{f \lambda} u_{d}^{g \rho} \gamma_{5} \gamma_{\mu} D_{\nu \rho} T_{A, n, e f g \lambda}$ & 104 & 398 \\
\hline$\epsilon^{a b c} \bar{B}_{a}{ }^{d} u_{b}{ }^{e \mu} u_{c}{ }^{f \nu} u_{e}{ }^{g \lambda} u_{g}{ }^{h \rho} \gamma_{5} \gamma_{\lambda} D_{\nu \rho} T_{A, n, d f h \mu}$ & & 78 & $i \epsilon^{a b c} \bar{B}_{a}^{d} f_{+b}{ }^{e \mu \nu} u_{c}{ }^{f \lambda} u_{d}^{g \rho} \gamma_{5} \gamma_{\mu} D_{\lambda \rho} T_{A, n, e f g \nu}$ & 105 & 399 \\
\hline$\epsilon^{a b c} \bar{B}_{a}^{d} u_{b}{ }^{e \mu} u_{c}{ }^{f \nu} u_{e}{ }^{g \lambda} u_{g}{ }^{h \rho} \gamma_{5} \gamma_{\rho} D_{\mu \nu} T_{A, n, d f h \lambda}$ & & 79 & $i \epsilon^{a b c} \bar{B}_{a}^{d} f_{+b}{ }^{e \mu \nu} u_{c}^{f \lambda} u_{d}^{g \rho} \gamma_{5} \gamma_{\lambda} D_{\mu \rho} T_{A, n, e f g \nu}$ & 106 & 400 \\
\hline$\epsilon^{a b c} \bar{B}_{a}{ }^{d} u_{b}{ }^{e \mu} u_{c}{ }^{f \nu} u_{e}{ }^{g \lambda} u_{g}{ }^{h \rho} \gamma_{5} \gamma_{\rho} D_{\mu \lambda} T_{A, n, d f h \nu}$ & & 80 & $i \epsilon^{a b c} \bar{B}_{a}^{d} f_{+b}{ }^{e \mu \nu} u_{c}{ }^{f \lambda} u_{d}^{g \rho} \gamma_{5} \gamma_{\rho} D_{\mu \lambda} T_{A, n, e f g \nu}$ & 107 & 401 \\
\hline$\epsilon^{a b c} \bar{B}_{a}{ }^{d} u_{b}{ }^{e \mu} u_{c}{ }^{f \nu} u_{e}{ }^{g \lambda} u_{g}{ }^{h \rho} \gamma_{5} \gamma_{\rho} D_{\nu \lambda} T_{A, n, d f h \mu}$ & & 81 & $i \epsilon^{a b c} \bar{B}_{a}^{d} f_{+b}{ }^{e \mu \nu} u_{c}^{f \lambda} u_{e}^{g \rho} \gamma_{5} \gamma_{\mu} D_{\nu \lambda} T_{A, n, d f g \rho}$ & 108 & 402 \\
\hline$\epsilon^{a b c} \bar{B}_{a}{ }^{d} u_{b}{ }^{e \mu} u_{d}{ }^{f \nu} u_{e}{ }^{g \lambda} u_{f}{ }^{h \rho} \gamma_{5} \gamma_{\mu} D_{\nu \lambda} T_{A, n, c g h \rho}$ & & 82 & $i \epsilon^{a b c} \bar{B}_{a}^{d} f_{+b}{ }^{e \mu \nu} u_{c}^{f \lambda} u_{e}^{g \rho} \gamma_{5} \gamma_{\mu} D_{\nu \rho} T_{A, n, d f g \lambda}$ & & 403 \\
\hline$\epsilon^{a b c} \bar{B}_{a}{ }^{d} u_{b}{ }^{e \mu} u_{d}{ }^{f \nu} u_{e}{ }^{g \lambda} u_{f}{ }^{h \rho} \gamma_{5} \gamma_{\mu} D_{\nu \rho} T_{A, n, c g h \lambda}$ & & 83 & $i \epsilon^{a b c} \bar{B}_{a}^{d} f_{+b}{ }^{e \mu \nu} u_{c}{ }^{f \lambda} u_{e}{ }^{g \rho} \gamma_{5} \gamma_{\mu} D_{\lambda \rho} T_{A, n, d f g \nu}$ & 109 & 404 \\
\hline$\epsilon^{a b c} \bar{B}_{a}{ }^{d} u_{b}{ }^{e \mu} u_{d}{ }^{f \nu} u_{e}{ }^{g \lambda} u_{f}{ }^{h \rho} \gamma_{5} \gamma_{\mu} D_{\lambda \rho} T_{A, n, c g h \nu}$ & & 84 & $i \epsilon^{a b c} \bar{B}_{a}^{d} f_{+b}{ }^{e \mu \nu} u_{c}{ }^{f \lambda} u_{e}{ }^{g \rho} \gamma_{5} \gamma_{\lambda} D_{\mu \rho} T_{A, n, d f g \nu}$ & 110 & 405 \\
\hline$\epsilon^{a b c} \bar{B}_{a}{ }^{d} u_{b}{ }^{e \mu} u_{d}{ }^{f \nu} u_{e}{ }^{g \lambda} u_{f}{ }^{h \rho} \gamma_{5} \gamma_{\lambda} D_{\mu \nu} T_{A, n, c g h \rho}$ & & 85 & $i \epsilon^{a b c} \bar{B}_{a}^{d} f_{+b}{ }^{e \mu \nu} u_{c}^{f \lambda} u_{e}^{g \rho} \gamma_{5} \gamma_{\rho} D_{\mu \lambda} T_{A, n, d f g \nu}$ & & 406 \\
\hline$\epsilon^{a b c} \bar{B}_{a}{ }^{d} u_{b}{ }^{e \mu} u_{d}{ }^{f \nu} u_{e}{ }^{g \lambda} u_{f}{ }^{h \rho} \gamma_{5} \gamma_{\lambda} D_{\mu \rho} T_{A, n, c g h \nu}$ & & 86 & $i \epsilon^{a b c} \bar{B}_{a}^{d} f_{+b}{ }^{e \mu \nu} u_{c}{ }^{f \lambda} u_{f}^{g \rho} \gamma_{5} \gamma_{\mu} D_{\nu \lambda} T_{A, n, \operatorname{deg} \rho}$ & 111 & 407 \\
\hline$\epsilon^{a b c} \bar{B}_{a}{ }^{d} u_{b}{ }^{e \mu} u_{d}{ }^{f \nu} u_{e}{ }^{g \lambda} u_{f}{ }^{h \rho} \gamma_{5} \gamma_{\lambda} D_{\nu \rho} T_{A, n, c g h \mu}$ & & 87 & $i \epsilon^{a b c} \bar{B}_{a}^{d} f_{+b}{ }^{e \mu \nu} u_{c}{ }^{f \lambda} u_{f}^{g \rho} \gamma_{5} \gamma_{\mu} D_{\nu \rho} T_{A, n, \operatorname{deg} \lambda}$ & 112 & 408 \\
\hline$\epsilon^{a b c} \bar{B}_{a}^{d} u_{b}{ }^{e \mu} u_{d}{ }^{f \nu} u_{e}{ }^{g \lambda} u_{g}{ }^{h \rho} \gamma_{5} \gamma_{\mu} D_{\nu \lambda} T_{A, n, c f h \rho}$ & & 88 & $i \epsilon^{a b c} \bar{B}_{a}^{d} f_{+b}{ }^{e \mu \nu} u_{c}^{f \lambda} u_{f}^{g \rho} \gamma_{5} \gamma_{\mu} D_{\lambda \rho} T_{A, n, \operatorname{deg} \nu}$ & 113 & 409 \\
\hline$\epsilon^{a b c} \bar{B}_{a}{ }^{d} u_{b}{ }^{e \mu} u_{d}{ }^{f \nu} u_{e}{ }^{g \lambda} u_{g}{ }^{h \rho} \gamma_{5} \gamma_{\mu} D_{\nu \rho} T_{A, n, c f h \lambda}$ & & 89 & $i \epsilon^{a b c} \bar{B}_{a}^{d} f_{+b}{ }^{e \mu \nu} u_{c}{ }^{f \lambda} u_{f}^{g \rho} \gamma_{5} \gamma_{\lambda} D_{\mu \rho} T_{A, n, \text { deg }}$ & 114 & 410 \\
\hline$\epsilon^{a b c} \bar{B}_{a}{ }^{d} u_{b}{ }^{e \mu} u_{d}{ }^{f \nu} u_{e}{ }^{g \lambda} u_{g}{ }^{h \rho} \gamma_{5} \gamma_{\nu} D_{\mu \lambda} T_{A, n, c f h \rho}$ & & 90 & $i \epsilon^{a b c} \bar{B}_{a}^{d} f_{+b}{ }^{e \mu \nu} u_{c}{ }^{f \lambda} u_{f}^{g \rho} \gamma_{5} \gamma_{\rho} D_{\mu \lambda} T_{A, n, \text { deg } \nu}$ & 115 & 411 \\
\hline$\epsilon^{a b c} \bar{B}_{a}{ }^{d} u_{b}{ }^{e \mu} u_{d}{ }^{f \nu} u_{e}{ }^{g \lambda} u_{g}{ }^{h \rho} \gamma_{5} \gamma_{\nu} D_{\mu \rho} T_{A, n, c f h \lambda}$ & & 91 & $i \epsilon^{a b c} \bar{B}_{a}^{d} f_{s,+}{ }^{\mu \nu} u_{b}{ }^{e}{ }_{\mu} u_{c}{ }^{f \lambda} \gamma_{5} \gamma_{\nu} T_{A, n, \text { def } \lambda}$ & 116 & \\
\hline$\epsilon^{a b c} \bar{B}_{a}{ }^{d} u_{b}{ }^{e \mu} u_{d}{ }^{f \nu} u_{e}{ }^{g \lambda} u_{g}{ }^{h \rho} \gamma_{5} \gamma_{\nu} D_{\lambda \rho} T_{A, n, c f h \mu}$ & & 92 & $i \epsilon^{a b c} \bar{B}_{a}{ }^{d} f_{s,+}{ }^{\mu \nu} u_{b}{ }^{e}{ }_{\mu} u_{c}{ }^{f \lambda} \gamma_{5} \gamma_{\lambda} T_{A, n, d e f \nu}$ & 117 & \\
\hline$\epsilon^{a b c} \bar{B}_{a}{ }^{d} u_{b}{ }^{e \mu} u_{d}{ }^{f \nu} u_{e}{ }^{g \lambda} u_{g}{ }^{h \rho} \gamma_{5} \gamma_{\lambda} D_{\mu \nu} T_{A, n, c f h \rho}$ & & 93 & $i \epsilon^{a b c} \bar{B}_{a}{ }^{d} f_{s,+}{ }^{\mu \nu} u_{b}{ }^{e}{ }_{\mu} u_{d}{ }^{f \lambda} \gamma_{5} \gamma_{\nu} T_{A, n, c e f \lambda}$ & 118 & \\
\hline$\epsilon^{a b c} \bar{B}_{a}{ }^{d} u_{b}{ }^{e \mu} u_{d}{ }^{f \nu} u_{e}{ }^{g \lambda} u_{g}{ }^{h \rho} \gamma_{5} \gamma_{\lambda} D_{\nu \rho} T_{A, n, c f h \mu}$ & & 94 & $i \epsilon^{a b c} \bar{B}_{a}{ }^{d} f_{s,+}{ }^{\mu \nu} u_{b}{ }^{e}{ }_{\mu} u_{d}{ }^{f \lambda} \gamma_{5} \gamma_{\lambda} T_{A, n, c e f \nu}$ & 119 & \\
\hline$\epsilon^{a b c} \bar{B}_{a}{ }^{d} u_{b}{ }^{e \mu} u_{e}{ }^{f \nu} u_{f}^{g \lambda} u_{g}^{h \rho} \gamma_{5} \gamma_{\mu} D_{\nu \lambda} T_{A, n, c d h \rho}$ & & 95 & $i \epsilon^{a b c} \bar{B}_{a}^{d} f_{s,+}^{\mu \nu} u_{b}{ }^{e \lambda} u_{d}{ }_{\lambda}{ }_{\lambda} \gamma_{5} \gamma_{\mu} T_{A, n, c e f \nu}$ & 120 & \\
\hline$\epsilon^{a b c} \bar{B}_{a}{ }^{d} u_{b}{ }^{e \mu} u_{e}{ }^{f \nu} u_{f}{ }^{g \lambda} u_{g}{ }^{h \rho} \gamma_{5} \gamma_{\mu} D_{\nu \rho} T_{A, n, c d h \lambda}$ & & 96 & $i \epsilon^{a b c} \bar{B}_{a}{ }^{d} f_{s,+}{ }^{\mu \nu} u_{b}{ }^{e \lambda} u_{c}{ }^{f \rho} \gamma_{5} \gamma_{\mu} D_{\nu \lambda} T_{A, n, \operatorname{def} \rho}$ & 121 & \\
\hline$\epsilon^{a b c} \bar{B}_{a}{ }^{d} u_{b}{ }^{e \mu} u_{e}{ }^{f \nu} u_{f}^{g \lambda} u_{g}^{h \rho} \gamma_{5} \gamma_{\mu} D_{\lambda \rho} T_{A, n, c d h \nu}$ & & 97 & $i \epsilon^{a b c} \bar{B}_{a}{ }^{d} f_{s,+}{ }^{\mu \nu} u_{b}{ }^{e \lambda} u_{c}{ }^{f \rho} \gamma_{5} \gamma_{\lambda} D_{\mu \rho} T_{A, n, \text { def } \nu}$ & 122 & \\
\hline$\epsilon^{a b c} \bar{B}_{a}{ }^{d} u_{b}{ }^{e \mu} u_{e}{ }^{f \nu} u_{f}{ }^{g \lambda} u_{g}{ }^{h \rho} \gamma_{5} \gamma_{\nu} D_{\mu \lambda} T_{A, n, c d h \rho}$ & & 98 & $i \epsilon^{a b c} \bar{B}_{a}{ }^{d} f_{s,+}{ }^{\mu \nu} u_{b}{ }^{e \lambda} u_{d}{ }^{f \rho} \gamma_{5} \gamma_{\mu} D_{\nu \lambda} T_{A, n, c e f \rho}$ & 123 & \\
\hline$\epsilon^{a b c} \bar{B}_{a}{ }^{d} u_{b}{ }^{e \mu} u_{e}{ }^{f \nu} u_{f}^{g \lambda} u_{g}^{h \rho} \gamma_{5} \gamma_{\nu} D_{\mu \rho} T_{A, n, c d h \lambda}$ & & 99 & $i \epsilon^{a b c} \bar{B}_{a}^{d} f_{s,+}^{\mu \nu} u_{b}^{e \lambda} u_{d}^{f \rho} \gamma_{5} \gamma_{\mu} D_{\lambda \rho} T_{A, n, c e f \nu}$ & 124 & \\
\hline$\epsilon^{a b c} \bar{B}_{a}{ }^{d} u_{b}{ }^{e \mu} u_{e}{ }^{f \nu} u_{f}^{g \lambda} u_{g}^{h \rho} \gamma_{5} \gamma_{\nu} D_{\lambda \rho} T_{A, n, c d h \mu}$ & & 100 & $i \epsilon^{a b c} \bar{B}_{a}^{d} f_{s,+}^{\mu \nu} u_{b}{ }^{e \lambda} u_{d}^{f \rho} \gamma_{5} \gamma_{\lambda} D_{\mu \rho} T_{A, n, c e f \nu}$ & 125 & \\
\hline$\epsilon^{a b c} \bar{B}_{a}{ }^{d} u_{b}{ }^{e \mu} u_{e}{ }^{f \nu} u_{f}^{g \lambda} u_{g}^{h \rho} \gamma_{5} \gamma_{\rho} D_{\mu \nu} T_{A, n, c d h \lambda}$ & & 101 & $i \epsilon^{a b c} \bar{B}_{a}^{d} f_{+b}{ }^{e \mu \nu} u_{d}^{f \lambda} u_{e}^{g \rho} \gamma_{5} \gamma_{\mu} D_{\nu \lambda} T_{A, n, c f g \rho}$ & & 412 \\
\hline$\epsilon^{a b c} \bar{B}_{a}{ }^{d} u_{b}{ }^{e \mu} u_{e}{ }^{f \nu} u_{f}^{g \lambda} u_{g}^{h \rho} \gamma_{5} \gamma_{\rho} D_{\mu \lambda} T_{A, n, c d h \nu}$ & & 102 & $i \epsilon^{a b c} \bar{B}_{a}^{d} f_{+b}{ }^{e \mu \nu} u_{d}^{f \lambda} u_{e}^{g \rho} \gamma_{5} \gamma_{\mu} D_{\nu \rho} T_{A, n, c f g \lambda}$ & & 413 \\
\hline$\varepsilon^{\mu \nu \lambda \rho} \epsilon^{a b c} \bar{B}_{a}{ }^{d} u_{b}{ }^{e}{ }_{\mu} u_{c}{ }^{f}{ }_{\nu} u_{d}{ }^{g}{ }_{\lambda} u_{e}{ }^{h \sigma} D_{\rho} T_{A, n, f g h \sigma}$ & 15 & 103 & $i \epsilon^{a b c} \bar{B}_{a}^{d} f_{+b}{ }^{e \mu \nu} u_{d}^{f \lambda} u_{e}^{g \rho} \gamma_{5} \gamma_{\mu} D_{\lambda \rho} T_{A, n, c f g \nu}$ & & 414 \\
\hline$\varepsilon^{\mu \nu \lambda \rho} \epsilon^{a b c} \bar{B}_{a}{ }^{d} u_{b}{ }_{\mu}{ }_{\mu} u_{c}{ }^{f}{ }_{\nu} u_{d}{ }^{g \sigma} u_{e}{ }_{\lambda}{ }_{\lambda} D_{\rho} T_{A, n, f g h \sigma}$ & & 104 & $i \epsilon^{a b c} \bar{B}_{a}^{d} f_{+b}{ }^{e \mu \nu} u_{d}^{f \lambda} u_{e}^{g \rho} \gamma_{5} \gamma_{\lambda} D_{\mu \rho} T_{A, n, c f g \nu}$ & & 415 \\
\hline$\varepsilon^{\mu \nu \lambda \rho} \epsilon^{a b c} \bar{B}_{a}{ }^{d} u_{b}{ }_{\mu}{ }_{\mu} u_{c}{ }^{f}{ }_{\nu} u_{d}{ }^{g \sigma} u_{g}{ }_{\lambda}{ }_{\lambda} D_{\rho} T_{A, n, e f h \sigma}$ & & 105 & $i \epsilon^{a b c} \bar{B}_{a}^{d} f_{+b}{ }^{e \mu \nu} u_{d}^{f \lambda} u_{e}^{g \rho} \gamma_{5} \gamma_{\rho} D_{\mu \lambda} T_{A, n, c f g \nu}$ & & 416 \\
\hline$\varepsilon^{\mu \nu \lambda \rho} \epsilon^{a b c} \bar{B}_{a}{ }^{d} u_{b}{ }^{e}{ }_{\mu} u_{c}{ }^{f}{ }_{\nu} u_{e}{ }^{g}{ }_{\lambda} u_{f}{ }^{h \sigma} D_{\rho} T_{A, n, d g h \sigma}$ & & 106 & $i \epsilon^{a b c} \bar{B}_{a}^{d} f_{+b}{ }^{e \mu \nu} u_{d}^{f \lambda} u_{f}^{g \rho} \gamma_{5} \gamma_{\mu} D_{\nu \lambda} T_{A, n, \text { ceg } \rho}$ & & 417 \\
\hline$\varepsilon^{\mu \nu \lambda \rho} \epsilon^{a b c} \bar{B}_{a}{ }^{d} u_{b}{ }^{e}{ }_{\mu} u_{c}{ }^{f}{ }_{\nu} u_{e}{ }^{g}{ }_{\lambda} u_{g}{ }^{h \sigma} D_{\rho} T_{A, n, d f h \sigma}$ & & 107 & $i \epsilon^{a b c} \bar{B}_{a}^{d} f_{+b}{ }^{e \mu \nu} u_{d}^{f \lambda} u_{f}^{g \rho} \gamma_{5} \gamma_{\mu} D_{\nu \rho} T_{A, n, \operatorname{ceg} \lambda}$ & & 418 \\
\hline$\varepsilon^{\mu \nu \lambda \rho} \epsilon^{a b c} \bar{B}_{a}{ }^{d} u_{b}{ }^{e}{ }_{\mu} u_{c}{ }^{f}{ }_{\nu} u_{e}{ }^{g \sigma} u_{g}{ }^{h}{ }_{\lambda} D_{\rho} T_{A, n, d f h \sigma}$ & & 108 & $i \epsilon^{a b c} \bar{B}_{a}^{d} f_{+b}{ }^{e \mu \nu} u_{d}{ }^{f \lambda} u_{f}{ }^{g \rho} \gamma_{5} \gamma_{\mu} D_{\lambda \rho} T_{A, n, c e g \nu}$ & & 419 \\
\hline$\varepsilon^{\mu \nu \lambda \rho} \epsilon^{a b c} \bar{B}_{a}{ }^{d} u_{b}{ }^{e}{ }_{\mu} u_{c}{ }^{f}{ }_{\nu} u^{g h}{ }_{\lambda} u_{h g}{ }^{\sigma} D_{\rho} T_{A, n, \operatorname{def} \sigma}$ & & 109 & $i \epsilon^{a b c} \bar{B}_{a}^{d} f_{+b}{ }^{e \mu \nu} u_{d}^{f \lambda} u_{f}^{g \rho} \gamma_{5} \gamma_{\lambda} D_{\mu \rho} T_{A, n, c e g \nu}$ & & 420 \\
\hline$\varepsilon^{\mu \nu \lambda \rho} \epsilon^{a b c} \bar{B}_{a}{ }^{d} u_{b}{ }_{\mu}{ }_{\mu} u_{c}{ }^{f \sigma} u_{e}{ }^{g}{ }_{\nu} u_{f}{ }_{\lambda}{ }_{\lambda} D_{\rho} T_{A, n, d g h \sigma}$ & & 110 & $i \epsilon^{a b c} \bar{B}_{a}^{d} f_{+b}{ }^{e \mu \nu} u_{d}{ }^{f \lambda} u_{f}{ }^{g \rho} \gamma_{5} \gamma_{\rho} D_{\mu \lambda} T_{A, n, c e g \nu}$ & & 421 \\
\hline$\varepsilon^{\mu \nu \lambda \rho} \epsilon^{a b c} \bar{B}_{a}{ }^{d} u_{b}{ }^{e}{ }_{\mu} u_{d}{ }^{f}{ }_{\nu} u_{e}{ }_{\lambda}{ }_{\lambda} u_{f}{ }^{h \sigma} D_{\rho} T_{A, n, c g h \sigma}$ & & 111 & $i \epsilon^{a b c} \bar{B}_{a}^{d} f_{+b}{ }^{e \mu \nu} u_{e}{ }^{f \lambda} u_{f}^{g \rho} \gamma_{5} \gamma_{\mu} D_{\nu \lambda} T_{A, n, c d g \rho}$ & & 422 \\
\hline$\varepsilon^{\mu \nu \lambda \rho} \epsilon^{a b c} \bar{B}_{a}{ }^{d} u_{b}{ }^{e}{ }_{\mu} u_{d}{ }^{f}{ }_{\nu} u_{e}{ }_{\lambda}{ }_{\lambda} u_{g}{ }^{h \sigma} D_{\rho} T_{A, n, c f h \sigma}$ & & 112 & $i \epsilon^{a b c} \bar{B}_{a}^{d} f_{+b}{ }^{e \mu \nu} u_{e}{ }^{f \lambda} u_{f}^{g \rho} \gamma_{5} \gamma_{\mu} D_{\nu \rho} T_{A, n, c d g \lambda}$ & & 423 \\
\hline$\varepsilon^{\mu \nu \lambda \rho} \epsilon^{a b c} \bar{B}_{a}{ }^{d} u_{b}{ }_{\mu}{ }_{\mu} u_{e}{ }_{\nu}{ }_{\nu} u_{f}{ }_{\lambda}^{g} u_{g}{ }^{h \sigma} D_{\rho} T_{A, n, c d h \sigma}$ & & 113 & $i \epsilon^{a b c} \bar{B}_{a}^{d} f_{+b}{ }^{e \mu \nu} u_{e}{ }^{f \lambda} u_{f}^{g \rho} \gamma_{5} \gamma_{\mu} D_{\lambda \rho} T_{A, n, c d g \nu}$ & & 424 \\
\hline$\varepsilon^{\mu \nu \lambda \rho} \epsilon^{a b c} \bar{B}_{a}{ }^{d} u_{b}{ }_{\mu}{ }_{\mu} u_{e}{ }^{f}{ }_{\nu} u_{f}{ }^{g \sigma} u_{g}{ }_{\lambda}{ }_{\lambda} D_{\rho} T_{A, n, c d h \sigma}$ & & 114 & $i \epsilon^{a b c} \bar{B}_{a}{ }^{d} f_{+b}{ }^{e \mu \nu} u_{e}{ }^{f \lambda} u_{f}^{g \rho} \gamma_{5} \gamma_{\lambda} D_{\mu \rho} T_{A, n, c d g \nu}$ & & 425 \\
\hline
\end{tabular}


TABLE VII. (Continued)

\begin{tabular}{|c|c|c|c|c|c|}
\hline$P_{n}^{\left(N_{f}, 4\right)}$ & $S U(2)$ & $S U(3)$ & $P_{n}^{\left(N_{f}, 4\right)}$ & $S U(2)$ & $S U(3)$ \\
\hline$\epsilon^{a b c} \bar{B}_{a}{ }^{d} u_{b}{ }^{e \mu} u_{c}{ }^{f}{ }_{\mu} f_{-e}{ }^{g \nu \lambda} D_{\nu} T_{A, n, d f g \lambda}$ & 16 & 115 & $i \epsilon^{a b c} \bar{B}_{a}{ }^{d} f_{+b}{ }^{e \mu \nu} u_{e}{ }^{f \lambda} u_{f}^{g \rho} \gamma_{5} \gamma_{\rho} D_{\mu \lambda} T_{A, n, c d g \nu}$ & & 426 \\
\hline$\epsilon^{a b c} \bar{B}_{a}{ }^{d} u_{b}{ }^{e \mu} u_{c}{ }^{f \nu} f_{-d^{\prime}{ }_{\mu}{ }^{\lambda} D_{\nu} T_{A, n, e f g \lambda}}$ & 17 & 116 & $i \epsilon^{a b c} \bar{B}_{a}^{d} f_{+b}^{e \mu \nu} u^{f g \lambda} u_{g f}{ }^{\rho} \gamma_{5} \gamma_{\mu} D_{\nu \lambda} T_{A, n, c d e \rho}$ & & 427 \\
\hline$\epsilon^{a b c} \bar{B}_{a}{ }^{d} u_{b}{ }^{e \mu} u_{c}{ }^{f \nu} f_{-d^{\prime}{ }_{\mu}{ }^{\lambda} D_{\lambda} T_{A, n, e f g \nu}}$ & 18 & 117 & $i \epsilon^{a b c} \bar{B}_{a}^{d} f_{+b}{ }^{e \mu \nu} u^{f g \lambda} u_{g f}{ }^{\rho} \gamma_{5} \gamma_{\mu} D_{\lambda \rho} T_{A, n, c d e \nu}$ & & 428 \\
\hline$\epsilon^{a b c} \bar{B}_{a}{ }^{d} u_{b}{ }^{e \mu} u_{c}{ }^{f \nu} f_{-e^{g}{ }_{\mu}{ }^{\lambda} D_{\nu} T_{A, n, d f g \lambda}}$ & 19 & 118 & $i \epsilon^{a b c} \bar{B}_{a}{ }^{d} f_{+b}{ }^{e \mu \nu} u^{f g \lambda} u_{g f}{ }^{\rho} \gamma_{5} \gamma_{\lambda} D_{\mu \rho} T_{A, n, c d e \nu}$ & & 429 \\
\hline$\epsilon^{a b c} \bar{B}_{a}{ }^{d} u_{b}{ }^{e \mu} u_{c}{ }^{f \nu} f_{-e^{g}{ }_{\mu}{ }^{\lambda} D_{\lambda} T_{A, n, d f g \nu}}$ & 20 & 119 & $i \epsilon^{a b c} \bar{B}_{a}^{d} f_{+}{ }^{e f \mu \nu} u_{b e}{ }^{\lambda} u_{c}{ }^{g \rho} \gamma_{5} \gamma_{\mu} D_{\nu \lambda} T_{A, n, d f g \rho}$ & & 430 \\
\hline$\epsilon^{a b c} \bar{B}_{a}{ }^{d} u_{b}{ }^{e \mu} u_{c}{ }^{f \nu} f_{-e^{g}{ }_{\nu}{ }^{\lambda}} D_{\mu} T_{A, n, d f g \lambda}$ & 21 & 120 & $i \epsilon^{a b c} \bar{B}_{a}^{d} f_{+}{ }^{e f \mu \nu} u_{b e^{\lambda}} u_{c}{ }^{g \rho} \gamma_{5} \gamma_{\mu} D_{\nu \rho} T_{A, n, d f g \lambda}$ & & 431 \\
\hline$\epsilon^{a b c} \bar{B}_{a}{ }^{d} u_{b}{ }^{e \mu} u_{c}{ }^{f \nu} f_{-e}{ }^{g}{ }_{\nu}{ }^{\lambda} D_{\lambda} T_{A, n, d f g \mu}$ & 22 & 121 & $i \epsilon^{a b c} \bar{B}_{a}{ }^{d} f_{+}{ }^{e f \mu \nu} u_{b e}{ }^{\lambda} u_{c}{ }^{g \rho} \gamma_{5} \gamma_{\mu} D_{\lambda \rho} T_{A, n, d f g \nu}$ & & 432 \\
\hline$\epsilon^{a b c} \bar{B}_{a}{ }^{d} u_{b}{ }^{e \mu} u_{d}{ }^{f} f_{-c}{ }^{g \nu \lambda} D_{\nu} T_{A, n, e f g \lambda}$ & 23 & 122 & $i \epsilon^{a b c} \bar{B}_{a}^{d} f_{+}{ }^{e f \mu \nu} u_{b e^{\lambda}} u_{c}{ }^{g \rho} \gamma_{5} \gamma_{\lambda} D_{\mu \rho} T_{A, n, d f g \nu}$ & & 433 \\
\hline$\epsilon^{a b c} \bar{B}_{a}{ }^{d} u_{b}{ }^{e \mu} u_{d}{ }^{f} f_{-e^{g \nu \lambda}} D_{\nu} T_{A, n, c f g \lambda}$ & & 123 & $i \epsilon^{a b c} \bar{B}_{a}^{d} f_{+}{ }^{e f \mu \nu} u_{b e^{\lambda} u_{c}{ }^{g \rho} \gamma_{5} \gamma_{\rho} D_{\mu \lambda} T_{A, n, d f g \nu}}$ & & 434 \\
\hline$\epsilon^{a b c} \bar{B}_{a}^{d} u_{b}{ }^{e \mu} u_{d}{ }^{f \nu} f_{-c}{ }^{g}{ }_{\mu}^{\lambda} D_{\nu} T_{A, n, e f g \lambda}$ & 24 & 124 & $i \epsilon^{a b c} \bar{B}_{a}^{d} f_{+}{ }^{e f \mu \nu} u_{b e}{ }^{\lambda} u_{d}{ }^{g \rho} \gamma_{5} \gamma_{\mu} D_{\nu \lambda} T_{A, n, c f g \rho}$ & & 435 \\
\hline$\epsilon^{a b c} \bar{B}_{a}{ }^{d} u_{b}{ }^{e \mu} u_{d}{ }^{f \nu} f_{-c^{\prime}{ }_{\mu}{ }^{\lambda} D_{\lambda} T_{A, n, e f g \nu}}$ & 25 & 125 & $i \epsilon^{a b c} \bar{B}_{a}^{d} f_{+}{ }^{e f \mu \nu} u_{b e}{ }^{\lambda} u_{d}^{g \rho} \gamma_{5} \gamma_{\mu} D_{\nu \rho} T_{A, n, c f g \lambda}$ & & 436 \\
\hline$\epsilon^{a b c} \bar{B}_{a}{ }^{d} u_{b}{ }^{e \mu} u_{d}{ }^{f \nu} f_{-e^{g}{ }_{\mu}{ }^{\lambda}} D_{\nu} T_{A, n, c f g \lambda}$ & & 126 & $i \epsilon^{a b c} \bar{B}_{a}{ }^{d} f_{+}{ }^{e f \mu \nu} u_{b e}{ }^{\lambda} u_{d}{ }^{g \rho} \gamma_{5} \gamma_{\mu} D_{\lambda \rho} T_{A, n, c f g \nu}$ & & 437 \\
\hline$\epsilon^{a b c} \bar{B}_{a}{ }^{d} u_{b}{ }^{e \mu} u_{d}{ }^{f \nu} f_{-e^{g}{ }_{\mu}{ }^{\lambda} D_{\lambda} T_{A, n, c f g \nu}}$ & & 127 & $i \epsilon^{a b c} \bar{B}_{a}{ }^{d} f_{+}{ }^{e f \mu \nu} u_{b e}{ }^{\lambda} u_{d}{ }^{g \rho} \gamma_{5} \gamma_{\lambda} D_{\mu \rho} T_{A, n, c f g \nu}$ & & 438 \\
\hline$\epsilon^{a b c} \bar{B}_{a}{ }^{d} u_{b}{ }^{e \mu} u_{d}{ }^{f \nu} f_{-e^{g}{ }_{\nu}{ }^{\lambda}} D_{\mu} T_{A, n, c f g \lambda}$ & & 128 & $i \epsilon^{a b c} \bar{B}_{a}^{d} f_{+}{ }^{e f \mu \nu} u_{b e}{ }^{\lambda} u_{d}^{g \rho} \gamma_{5} \gamma_{\rho} D_{\mu \lambda} T_{A, n, c f g \nu}$ & & 439 \\
\hline$\epsilon^{a b c} \bar{B}_{a}{ }^{d} u_{b}{ }^{e \mu} u_{d}{ }^{f \nu} f_{-e^{g}{ }_{\nu}{ }^{\lambda}} D_{\lambda} T_{A, n, c f g \mu}$ & & 129 & $i \epsilon^{a b c} \bar{B}_{a}^{d} f_{+}{ }^{e f \mu \nu} u_{b e}{ }^{\lambda} u_{f}^{g \rho} \gamma_{5} \gamma_{\mu} D_{\nu \lambda} T_{A, n, c d g \rho}$ & & 440 \\
\hline$\epsilon^{a b c} \bar{B}_{a}{ }^{d} u_{b}{ }^{e \mu} u_{e}{ }_{\mu}^{f} f_{-c}{ }^{g \nu \lambda} D_{\nu} T_{A, n, d f g \lambda}$ & & 130 & $i \epsilon^{a b c} \bar{B}_{a}^{d} f_{+}{ }^{e f \mu \nu} u_{b e}{ }^{\lambda} u_{f}{ }^{g \rho} \gamma_{5} \gamma_{\mu} D_{\nu \rho} T_{A, n, c d g \lambda}$ & & 441 \\
\hline$\epsilon^{a b c} \bar{B}_{a}{ }^{d} u_{b}{ }^{e \mu} u_{e}{ }_{\mu} f_{-d}{ }^{g \nu \lambda} D_{\nu} T_{A, n, c f g \lambda}$ & & 131 & $i \epsilon^{a b c} \bar{B}_{a}^{d} f_{+}{ }^{e f \mu \nu} u_{b e}{ }^{\lambda} u_{f}^{g \rho} \gamma_{5} \gamma_{\mu} D_{\lambda \rho} T_{A, n, c d g \nu}$ & & 442 \\
\hline$\epsilon^{a b c} \bar{B}_{a}{ }^{d} u_{b}{ }^{e \mu} u_{e}{ }_{\mu}^{f} f_{-f}{ }^{g \nu \lambda} D_{\nu} T_{A, n, c d g \lambda}$ & & 132 & $i \epsilon^{a b c} \bar{B}_{a}^{d} f_{+}{ }^{e f \mu \nu} u_{b e}{ }^{\lambda} u_{f}{ }^{g \rho} \gamma_{5} \gamma_{\lambda} D_{\mu \rho} T_{A, n, c d g \nu}$ & & 443 \\
\hline$\epsilon^{a b c} \bar{B}_{a}{ }^{d} u_{b}{ }^{e \mu} u_{e}{ }^{f \nu} f_{-c}{ }^{g}{ }_{\mu}^{\lambda} D_{\nu} T_{A, n, d f g \lambda}$ & & 133 & $i \epsilon^{a b c} \bar{B}_{a}{ }^{d} f_{+}{ }^{e f \mu \nu} u_{b e}{ }^{\lambda} u_{f}^{g \rho} \gamma_{5} \gamma_{\rho} D_{\mu \lambda} T_{A, n, c d g \nu}$ & & 444 \\
\hline$\epsilon^{a b c} \bar{B}_{a}{ }^{d} u_{b}{ }^{e \mu} u_{e}{ }^{f \nu} f_{-c}{ }^{g}{ }_{\mu}{ }^{\lambda} D_{\lambda} T_{A, n, d f g \nu}$ & & 134 & $i \epsilon^{a b c} \bar{B}_{a}^{d} f_{+}{ }^{e f \mu \nu} u_{b}{ }^{g \lambda} u_{g e}{ }^{\rho} \gamma_{5} \gamma_{\mu} D_{\nu \lambda} T_{A, n, c d f \rho}$ & & 445 \\
\hline$\epsilon^{a b c} \bar{B}_{a}{ }^{d} u_{b}{ }^{e \mu} u_{e}{ }^{f \nu} f_{-c}{ }^{g}{ }_{\nu}{ }^{\lambda} D_{\mu} T_{A, n, d f g \lambda}$ & & 135 & $i \epsilon^{a b c} \bar{B}_{a}{ }^{d} f_{+}{ }^{e f \mu \nu} u_{b}{ }^{g \lambda} u_{g e}{ }^{\rho} \gamma_{5} \gamma_{\lambda} D_{\mu \rho} T_{A, n, c d f \nu}$ & & 446 \\
\hline$\epsilon^{a b c} \bar{B}_{a}{ }^{d} u_{b}{ }^{e \mu} u_{e}{ }^{f \nu} f_{-c}{ }^{g}{ }_{\nu}{ }^{\lambda} D_{\lambda} T_{A, n, d f g \mu}$ & & 136 & $i \varepsilon^{\mu \nu \lambda \rho} \epsilon^{a b c} \bar{B}_{a}{ }^{d} f_{+b}{ }^{e}{ }_{\mu \nu} u_{c}{ }^{f}{ }_{\lambda} u_{d}{ }^{g \sigma} D_{\rho} T_{A, n, e f g \sigma}$ & 126 & 447 \\
\hline$\epsilon^{a b c} \bar{B}_{a}{ }^{d} u_{b}{ }^{e \mu} u_{e}{ }^{f \nu} f_{-d^{\prime}{ }_{\mu}{ }^{\lambda} D_{\nu} T_{A, n, c f g \lambda}}$ & & 137 & $i \varepsilon^{\mu \nu \lambda \rho} \epsilon^{a b c} \bar{B}_{a}{ }^{d} f_{+b}{ }_{\mu \nu} u_{c}{ }^{f}{ }_{\lambda} u_{e}{ }^{g \sigma} D_{\rho} T_{A, n, d f g \sigma}$ & 127 & 448 \\
\hline$\epsilon^{a b c} \bar{B}_{a}{ }^{d} u_{b}{ }^{e \mu} u_{e}{ }^{f \nu} f_{-d^{\prime}{ }_{\mu}{ }^{\lambda} D_{\lambda} T_{A, n, c f g \nu}}$ & & 138 & $i \varepsilon^{\mu \nu \lambda \rho} \epsilon^{a b c} \bar{B}_{a}{ }^{d} f_{+b}{ }^{e}{ }_{\mu \nu} u_{c}{ }^{f}{ }_{\lambda} u_{f}^{g \sigma} D_{\rho} T_{A, n, \operatorname{deg} \sigma}$ & 128 & 449 \\
\hline$\epsilon^{a b c} \bar{B}_{a}{ }^{d} u_{b}{ }^{e \mu} u_{e}{ }^{f \nu} f_{-d^{\prime}{ }_{\nu}{ }^{\lambda} D_{\mu} T_{A, n, c f g \lambda}}$ & & 139 & $i \varepsilon^{\mu \nu \lambda \rho} \epsilon^{a b c} \bar{B}_{a}{ }^{d} f_{+b}{ }^{e}{ }_{\mu \nu} u_{c}{ }^{f \sigma} u_{d}{ }_{\lambda} D_{\rho} T_{A, n, e f g \sigma}$ & 129 & 450 \\
\hline$\epsilon^{a b c} \bar{B}_{a}{ }^{d} u_{b}{ }^{e \mu} u_{e}{ }^{f \nu} f_{-d^{\prime}{ }_{\nu}{ }^{\lambda} D_{\lambda} T_{A, n, c f g \mu}}$ & & 140 & $i \varepsilon^{\mu \nu \lambda \rho} \epsilon^{a b c} \bar{B}_{a}{ }^{d} f_{+b}{ }^{e}{ }_{\mu \nu} u_{c}{ }^{f \sigma} u_{e}{ }_{\lambda} D_{\rho} T_{A, n, d f g \sigma}$ & & 451 \\
\hline$\epsilon^{a b c} \bar{B}_{a}{ }^{d} u_{b}{ }^{e \mu} u_{e}{ }^{f \nu} f_{-f_{\mu}}^{g}{ }^{\lambda} D_{\nu} T_{A, n, c d g \lambda}$ & & 141 & $i \varepsilon^{\mu \nu \lambda \rho} \epsilon^{a b c} \bar{B}_{a}{ }^{d} f_{+b}{ }^{e}{ }_{\mu \nu} u_{c}{ }^{f \sigma} u_{f}{ }_{\lambda}{ }_{\lambda} D_{\rho} T_{A, n, \operatorname{deg} \sigma}$ & 130 & 452 \\
\hline$\epsilon^{a b c} \bar{B}_{a}{ }^{d} u_{b}{ }^{e \mu} u_{e}{ }^{f \nu} f_{-f_{\mu}}^{g \lambda}{ }^{\lambda} D_{\lambda} T_{A, n, c d g \nu}$ & & 142 & $i \varepsilon^{\mu \nu \lambda \rho} \epsilon^{a b c} \bar{B}_{a}{ }^{d} f_{s,+\mu \nu} u_{b}{ }^{e}{ }_{\lambda} u_{c}{ }^{f \sigma} D_{\rho} T_{A, n, \operatorname{def} \sigma}$ & 131 & \\
\hline$\epsilon^{a b c} \bar{B}_{a}{ }^{d} u_{b}{ }^{e \mu} u_{e}{ }^{f \nu} f_{-f{ }^{g}{ }_{\nu} \lambda} D_{\mu} T_{A, n, c d g \lambda}$ & & 143 & $i \varepsilon^{\mu \nu \lambda \rho} \epsilon^{a b c} \bar{B}_{a}{ }^{d} f_{s,+\mu \nu} u_{b}{ }_{\lambda}{ }_{\lambda} u_{d}{ }^{f \sigma} D_{\rho} T_{A, n, c e f \sigma}$ & 132 & \\
\hline$\epsilon^{a b c} \bar{B}_{a}{ }^{d} u_{b}{ }^{e \mu} u_{e}{ }^{f \nu} f_{-f^{g}{ }_{\nu} \lambda} D_{\lambda} T_{A, n, c d g \mu}$ & & 144 & $i \varepsilon^{\mu \nu \lambda \rho} \epsilon^{a b c} \bar{B}_{a}{ }^{d} f_{+b}{ }^{e}{ }_{\mu \nu} u_{d}{ }^{f}{ }_{\lambda} u_{e}{ }^{g \sigma} D_{\rho} T_{A, n, c f g \sigma}$ & & 453 \\
\hline$\epsilon^{a b c} \bar{B}_{a}{ }^{d} u_{b}{ }^{e \mu} u^{f g}{ }_{\mu} f_{-c f}{ }^{\nu \lambda} D_{\nu} T_{A, n, \operatorname{deg} \lambda}$ & 26 & 145 & $i \varepsilon^{\mu \nu \lambda \rho} \epsilon^{a b c} \bar{B}_{a}{ }^{d} f_{+b}{ }_{\mu \nu} u_{d}{ }^{f}{ }_{\lambda} u_{f}^{g \sigma} D_{\rho} T_{A, n, \text { ceg } \sigma}$ & & 454 \\
\hline$\epsilon^{a b c} \bar{B}_{a}{ }^{d} u_{b}{ }^{e \mu} u^{f g}{ }_{\mu} f_{-d f}{ }^{\nu \lambda} D_{\nu} T_{A, n, \operatorname{ceg} \lambda}$ & & 146 & $i \varepsilon^{\mu \nu \lambda \rho} \epsilon^{a b c} \bar{B}_{a}{ }^{d} f_{+b}{ }^{e}{ }_{\mu \nu} u_{d}{ }^{f \sigma} u_{e}{ }_{\lambda} D_{\rho} T_{A, n, c f g \sigma}$ & & 455 \\
\hline$\epsilon^{a b c} \bar{B}_{a}{ }^{d} u_{b}{ }^{e \mu} u^{f g}{ }_{\mu} f_{-e f}{ }^{\nu \lambda} D_{\nu} T_{A, n, c d g \lambda}$ & & 147 & $i \varepsilon^{\mu \nu \lambda \rho} \epsilon^{a b c} \bar{B}_{a}{ }^{d} f_{+b}{ }^{e}{ }_{\mu \nu} u_{d}{ }^{f \sigma} u_{f}{ }_{\lambda}{ }_{\lambda} D_{\rho} T_{A, n, c e g \sigma}$ & & 456 \\
\hline$\epsilon^{a b c} \bar{B}_{a}{ }^{d} u_{b}{ }^{e \mu} u^{f g}{ }_{\mu} f_{-g f}{ }^{\nu \lambda} D_{\nu} T_{A, n, c d e \lambda}$ & & 148 & $i \varepsilon^{\mu \nu \lambda \rho} \epsilon^{a b c} \bar{B}_{a}{ }^{d} f_{+b}{ }^{e}{ }_{\mu \nu} u_{e}{ }_{\lambda}{ }_{\lambda} u_{f}^{g \sigma} D_{\rho} T_{A, n, c d g \sigma}$ & & 457 \\
\hline$\epsilon^{a b c} \bar{B}_{a}{ }^{d} u_{b}{ }^{e \mu} u^{f g \nu} f_{-c f \mu}{ }^{\lambda} D_{\nu} T_{A, n, \operatorname{deg} \lambda}$ & 27 & 149 & $i \varepsilon^{\mu \nu \lambda \rho} \epsilon^{a b c} \bar{B}_{a}{ }^{d} f_{+b}{ }^{e}{ }_{\mu \nu} u_{e}{ }^{f \sigma} u_{f}{ }_{\lambda} D_{\rho} T_{A, n, c d g \sigma}$ & & 458 \\
\hline$\epsilon^{a b c} \bar{B}_{a}{ }^{d} u_{b}{ }^{e \mu} u^{f g \nu} f_{-c f \mu}{ }^{\lambda} D_{\lambda} T_{A, n, \operatorname{deg} \nu}$ & 28 & 150 & $i \varepsilon^{\mu \nu \lambda \rho} \epsilon^{a b c} \bar{B}_{a}{ }^{d} f_{+b}{ }_{\mu \nu} u^{f g}{ }_{\lambda} u_{g f}{ }^{\sigma} D_{\rho} T_{A, n, c d e \sigma}$ & & 459 \\
\hline$\epsilon^{a b c} \bar{B}_{a}{ }^{d} u_{b}{ }^{e \mu} u^{f g \nu} f_{-c f \nu}{ }^{\lambda} D_{\mu} T_{A, n, \operatorname{deg} \lambda}$ & & 151 & $i \varepsilon^{\mu \nu \lambda \rho} \epsilon^{a b c} \bar{B}_{a}{ }^{d} f_{+}{ }^{e f}{ }_{\mu \nu} u_{b e \lambda} u_{c}{ }^{g \sigma} D_{\rho} T_{A, n, d f g \sigma}$ & & 460 \\
\hline$\epsilon^{a b c} \bar{B}_{a}{ }^{d} u_{b}{ }^{e \mu} u^{f g \nu} f_{-c f \nu}{ }^{\lambda} D_{\lambda} T_{A, n, \operatorname{deg} \mu}$ & & 152 & $i \varepsilon^{\mu \nu \lambda \rho} \epsilon^{a b c} \bar{B}_{a}{ }^{d} f_{+}{ }^{e f}{ }_{\mu \nu} u_{b e \lambda} u_{d}{ }^{g \sigma} D_{\rho} T_{A, n, c f g \sigma}$ & & 461 \\
\hline$\epsilon^{a b c} \bar{B}_{a}{ }^{d} u_{b}{ }^{e \mu} u^{f g \nu} f_{-d f \mu}{ }^{\lambda} D_{\nu} T_{A, n, c e g \lambda}$ & & 153 & $i \varepsilon^{\mu \nu \lambda \rho} \epsilon^{a b c} \bar{B}_{a}^{d} f_{+}{ }^{e f}{ }_{\mu \nu} u_{b e \lambda} u_{f}^{g \sigma} D_{\rho} T_{A, n, c d g \sigma}$ & & 462 \\
\hline$\epsilon^{a b c} \bar{B}_{a}{ }^{d} u_{b}{ }^{e \mu} u^{f g \nu} f_{-d f \mu}{ }^{\lambda} D_{\lambda} T_{A, n, c e g \nu}$ & & 154 & $i \varepsilon^{\mu \nu \lambda \rho} \epsilon^{a b c} \bar{B}_{a}{ }^{d} f_{+}{ }^{e f}{ }_{\mu \nu} u_{b e}{ }^{\sigma} u_{c}{ }_{\lambda}{ }_{\lambda} D_{\rho} T_{A, n, d f g \sigma}$ & & 463 \\
\hline$\epsilon^{a b c} \bar{B}_{a}{ }^{d} u_{b}{ }^{e \mu} u^{f g \nu} f_{-d f \nu}{ }^{\lambda} D_{\mu} T_{A, n, c e g \lambda}$ & & 155 & $i \varepsilon^{\mu \nu \lambda \rho} \epsilon^{a b c} \bar{B}_{a}{ }^{d} f_{+}{ }^{e f}{ }_{\mu \nu} u_{b e}{ }^{\sigma} u_{d}{ }_{\lambda} D_{\rho} T_{A, n, c f g \sigma}$ & & 464 \\
\hline$\epsilon^{a b c} \bar{B}_{a}{ }^{d} u_{b}{ }^{e \mu} u^{f g \nu} f_{-d f \nu}{ }^{\lambda} D_{\lambda} T_{A, n, c e g \mu}$ & & 156 & $i \varepsilon^{\mu \nu \lambda \rho} \epsilon^{a b c} \bar{B}_{a}{ }^{d} f_{+}{ }^{e f}{ }_{\mu \nu} u_{b e}{ }^{\sigma} u_{f}^{g}{ }_{\lambda} D_{\rho} T_{A, n, c d g \sigma}$ & & 465 \\
\hline$\epsilon^{a b c} \bar{B}_{a}{ }^{d} u_{b}{ }^{e \mu} u^{f g \nu} f_{-e f \mu}{ }^{\lambda} D_{\nu} T_{A, n, c d g \lambda}$ & & 157 & $i \varepsilon^{\mu \nu \lambda \rho} \epsilon^{a b c} \bar{B}_{a}{ }^{d} f_{+}{ }^{e f}{ }_{\mu \nu} u_{b}{ }_{\lambda}{ }_{\lambda} u_{g e}{ }^{\sigma} D_{\rho} T_{A, n, c d f \sigma}$ & & 466 \\
\hline$\epsilon^{a b c} \bar{B}_{a}{ }^{d} u_{b}{ }^{e \mu} u^{f g \nu} f_{-e f \mu}{ }^{\lambda} D_{\lambda} T_{A, n, c d g \nu}$ & & 158 & $i \epsilon^{a b c} \bar{B}_{a}{ }^{d} f_{+b}{ }^{e \mu \nu} f_{-c}^{f}{ }_{\mu}^{\lambda} D_{\nu} T_{A, n, \operatorname{def} \lambda}$ & 133 & 467 \\
\hline$\epsilon^{a b c} \bar{B}_{a}{ }^{d} u_{b}{ }^{e \mu} u^{f g \nu} f_{-e f \nu}{ }^{\lambda} D_{\mu} T_{A, n, c d g \lambda}$ & & 159 & $i \epsilon^{a b c} \bar{B}_{a}{ }^{d} f_{+b}{ }^{e \mu \nu} f_{-c}{ }^{f}{ }_{\mu}^{\lambda} D_{\lambda} T_{A, n, \text { def } \nu}$ & 134 & 468 \\
\hline$\epsilon^{a b c} \bar{B}_{a}{ }^{d} u_{b}{ }^{e \mu} u^{f g \nu} f_{-e f \nu}{ }^{\lambda} D_{\lambda} T_{A, n, c d g \mu}$ & & 160 & $i \epsilon^{a b c} \bar{B}_{a}^{d} f_{+b}^{e \mu \nu} f_{-d}{ }^{f}{ }_{\mu}^{\lambda} D_{\nu} T_{A, n, c e f \lambda}$ & 135 & 469 \\
\hline$\epsilon^{a b c} \bar{B}_{a}{ }^{d} u_{b}{ }^{e \mu} u^{f g \nu} f_{-g f \mu}{ }^{\lambda} D_{\nu} T_{A, n, c d e \lambda}$ & & 161 & $i \epsilon^{a b c} \bar{B}_{a}^{d} f_{+b}^{e \mu \nu} f_{-d^{f}{ }_{\mu}{ }^{\lambda} D_{\lambda} T_{A, n, c e f \nu}}$ & 136 & 470 \\
\hline
\end{tabular}


TABLE VII. (Continued)

\begin{tabular}{|c|c|c|c|c|c|}
\hline$P_{n}^{\left(N_{f}, 4\right)}$ & $S U(2)$ & $S U(3)$ & $P_{n}^{\left(N_{f}, 4\right)}$ & $S U(2)$ & $S U(3)$ \\
\hline$\epsilon^{a b c} \bar{B}_{a}{ }^{d} u_{b}{ }^{e \mu} u^{f g \nu} f_{-g f \mu}{ }^{\lambda} D_{\lambda} T_{A, n, c d e \nu}$ & & 162 & $i \epsilon^{a b c} \bar{B}_{a}{ }^{d} f_{+b}{ }^{e \mu \nu} f_{-e}{ }^{f}{ }_{\mu}^{\lambda} D_{\nu} T_{A, n, c d f \lambda}$ & & 471 \\
\hline$\epsilon^{a b c} \bar{B}_{a}{ }^{d} u_{b}{ }^{e \mu} u^{f g \nu} f_{-g f \nu}{ }^{\lambda} D_{\mu} T_{A, n, c d e \lambda}$ & & 163 & $i \epsilon^{a b c} \bar{B}_{a}^{d} f_{+b}{ }^{e \mu \nu} f_{-e^{f}{ }_{\mu}{ }^{\lambda} D_{\lambda} T_{A, n, c d f \nu}}$ & & 472 \\
\hline$\epsilon^{a b c} \bar{B}_{a}{ }^{d} u_{b}{ }^{e \mu} u^{f g \nu} f_{-g f \nu}{ }^{\lambda} D_{\lambda} T_{A, n, c d e \mu}$ & & 164 & $i \epsilon^{a b c} \bar{B}_{a}^{d} f_{+}{ }^{e f \mu \nu} f_{-b e \mu}{ }^{\lambda} D_{\nu} T_{A, n, c d f \lambda}$ & & 473 \\
\hline$\epsilon^{a b c} \bar{B}_{a}{ }^{d} u_{b}{ }^{e \mu} u_{c}{ }^{f \nu} f_{-e}{ }^{g \lambda \rho} D_{\mu \nu \lambda} T_{A, n, d f g \rho}$ & 29 & 165 & $i \epsilon^{a b c} \bar{B}_{a}^{d} f_{+}{ }^{e f \mu \nu} f_{-b e \mu}{ }^{\lambda} D_{\lambda} T_{A, n, c d f \nu}$ & & 474 \\
\hline$\epsilon^{a b c} \bar{B}_{a}{ }^{d} u_{b}{ }^{e \mu} u_{d}{ }^{f \nu} f_{-c}{ }^{g \lambda \rho} D_{\mu \nu \lambda} T_{A, n, \text { ef } g \rho}$ & 30 & 166 & $\epsilon^{a b c} \bar{B}_{a}{ }^{d} f_{+b}{ }^{e \mu \nu} f_{-c}{ }^{f \lambda \rho} \sigma_{\mu \nu} D_{\lambda} T_{A, n, \operatorname{def} \rho}$ & 137 & 475 \\
\hline$\epsilon^{a b c} \bar{B}_{a}{ }^{d} u_{b}{ }^{e \mu} u_{d}{ }^{f \nu} f_{-e}{ }^{g \lambda \rho} D_{\mu \nu \lambda} T_{A, n, c f g \rho}$ & & 167 & $\epsilon^{a b c} \bar{B}_{a}^{d} f_{+b}{ }^{e \mu \nu} f_{-c}{ }^{f \lambda \rho} \sigma_{\mu \lambda} D_{\nu} T_{A, n, d e f \rho}$ & 138 & 476 \\
\hline$\epsilon^{a b c} \bar{B}_{a}{ }^{d} u_{b}{ }^{e \mu} u_{e}{ }^{f \nu} f_{-c}{ }^{g \lambda \rho} D_{\mu \nu \lambda} T_{A, n, d f g \rho}$ & & 168 & $\epsilon^{a b c} \bar{B}_{a}{ }^{d} f_{+b}{ }^{e \mu \nu} f_{-d}^{f \lambda \rho} \sigma_{\mu \nu} D_{\lambda} T_{A, n, c e f \rho}$ & 139 & 477 \\
\hline$\epsilon^{a b c} \bar{B}_{a}{ }^{d} u_{b}{ }^{e \mu} u_{e}{ }^{f \nu} f_{-d}{ }^{g \lambda \rho} D_{\mu \nu \lambda} T_{A, n, c f g \rho}$ & & 169 & $\epsilon^{a b c} \bar{B}_{a}{ }^{d} f_{+b}{ }^{e \mu \nu} f_{-d}{ }^{f \lambda \rho} \sigma_{\mu \lambda} D_{\nu} T_{A, n, c e f \rho}$ & 140 & 478 \\
\hline$\epsilon^{a b c} \bar{B}_{a}{ }^{d} u_{b}{ }^{e \mu} u_{e}{ }^{f \nu} f_{-f}{ }^{g \lambda \rho} D_{\mu \nu \lambda} T_{A, n, c d g \rho}$ & & 170 & $i \epsilon^{a b c} \bar{B}_{a}{ }^{d} f_{s,+}{ }^{\mu \nu} f_{-b}{ }^{e}{ }_{\mu}^{\lambda} D_{\nu} T_{A, n, c d e \lambda}$ & 141 & \\
\hline$\epsilon^{a b c} \bar{B}_{a}^{d} u_{b}{ }^{e \mu} u^{f g \nu} f_{-c f}^{\lambda \rho} D_{\mu \nu \lambda} T_{A, n, \operatorname{deg} \rho}$ & 31 & 171 & $i \epsilon^{a b c} \bar{B}_{a}^{d} f_{s,+}{ }^{\mu \nu} f_{-b}{ }^{e}{ }_{\mu}^{\lambda} D_{\lambda} T_{A, n, c d e \nu}$ & 142 & \\
\hline$\epsilon^{a b c} \bar{B}_{a}{ }^{d} u_{b}{ }^{e \mu} u^{f g \nu} f_{-d f}^{\lambda \rho} D_{\mu \nu \lambda} T_{A, n, c e g \rho}$ & & 172 & $\epsilon^{a b c} \bar{B}_{a}{ }^{d} f_{s,+}{ }^{\mu \nu} f_{-b}{ }^{e \lambda \rho} \sigma_{\mu \lambda} D_{\nu} T_{A, n, c d e \rho}$ & 143 & \\
\hline$\epsilon^{a b c} \bar{B}_{a}{ }^{d} u_{b}{ }^{e \mu} u^{f g \nu} f_{-e f}{ }^{\lambda \rho} D_{\mu \nu \lambda} T_{A, n, c d g \rho}$ & & 173 & $\epsilon^{a b c} \bar{B}_{a}^{d} f_{+b}^{e \mu \nu} f_{-e}^{f \lambda \rho} \sigma_{\mu \nu} D_{\lambda} T_{A, n, c d f \rho}$ & & 479 \\
\hline$\epsilon^{a b c} \bar{B}_{a}{ }^{d} u_{b}{ }^{e \mu} u^{f g \nu} f_{-g f}{ }^{\lambda \rho} D_{\mu \nu \lambda} T_{A, n, c d e \rho}$ & & 174 & $\epsilon^{a b c} \bar{B}_{a}^{d} f_{+b}^{e \mu \nu} f_{-e}^{f \lambda \rho} \sigma_{\mu \lambda} D_{\nu} T_{A, n, c d f \rho}$ & & 480 \\
\hline$i \epsilon^{a b c} \bar{B}_{a}{ }^{d} u_{b}{ }^{e \mu} u_{c}{ }^{f \nu} f_{-d}^{g \lambda \rho} \sigma_{\mu \nu} D_{\lambda} T_{A, n, e f g \rho}$ & 32 & 175 & $\epsilon^{a b c} \bar{B}_{a}{ }^{d} f_{+}{ }^{e f \mu \nu} f_{-b e}{ }^{\lambda \rho} \sigma_{\mu \nu} D_{\lambda} T_{A, n, c d f \rho}$ & & 481 \\
\hline$i \epsilon^{a b c} \bar{B}_{a}{ }^{d} u_{b}{ }^{e \mu} u_{c}{ }^{f \nu} f_{-d^{g \lambda \rho}} \sigma_{\mu \lambda} D_{\nu} T_{A, n, \text { ef } g \rho}$ & 33 & 176 & $\epsilon^{a b c} \bar{B}_{a}{ }^{d} f_{+}{ }^{e f \mu \nu} f_{-b e^{\lambda \rho} \sigma_{\mu \lambda} D_{\nu} T_{A, n, c d f \rho}}$ & & 482 \\
\hline$i \epsilon^{a b c} \bar{B}_{a}{ }^{d} u_{b}{ }^{e \mu} u_{c}{ }^{f \nu} f_{-e^{g \lambda \rho}} \sigma_{\mu \nu} D_{\lambda} T_{A, n, d f g \rho}$ & 34 & 177 & $i \epsilon^{a b c} \bar{B}_{a}{ }^{d} f_{+b}{ }^{e \mu \nu} h_{c}{ }^{f}{ }_{\mu}^{\lambda} D_{\nu} T_{A, n, \operatorname{def} \lambda}$ & 144 & 483 \\
\hline$i \epsilon^{a b c} \bar{B}_{a}{ }^{d} u_{b}{ }^{e \mu} u_{c}{ }^{f \nu} f_{-e^{g \lambda \rho}} \sigma_{\mu \lambda} D_{\nu} T_{A, n, d f g \rho}$ & 35 & 178 & $i \epsilon^{a b c} \bar{B}_{a}{ }^{d} f_{+b}{ }^{e \mu \nu} h_{c}{ }^{f}{ }_{\mu}^{\lambda} D_{\lambda} T_{A, n, \text { def } \nu}$ & 145 & 484 \\
\hline$i \epsilon^{a b c} \bar{B}_{a}^{d} u_{b}^{e \mu} u_{c}^{f \nu} f_{-e^{g \lambda \rho}} \sigma_{\mu \lambda} D_{\rho} T_{A, n, d f g \nu}$ & 36 & 179 & $i \epsilon^{a b c} \bar{B}_{a}^{d} f_{+b}{ }^{e \mu \nu} h_{d}{ }^{f}{ }_{\mu}^{\lambda} D_{\nu} T_{A, n, c e f \lambda}$ & 146 & 485 \\
\hline$i \epsilon^{a b c} \bar{B}_{a}{ }^{d} u_{b}{ }^{e \mu} u_{c}{ }^{f \nu} f_{-e^{g \lambda \rho}} \sigma_{\nu \lambda} D_{\mu} T_{A, n, d f g \rho}$ & 37 & 180 & $i \epsilon^{a b c} \bar{B}_{a}{ }^{d} f_{+b}{ }^{e \mu \nu} h_{d}{ }_{\mu}^{f}{ }^{\lambda} D_{\lambda} T_{A, n, c e f \nu}$ & 147 & 486 \\
\hline$i \epsilon^{a b c} \bar{B}_{a}^{d} u_{b}^{e \mu} u_{d}^{f \nu} f_{-c}^{g \lambda \rho} \sigma_{\mu \lambda} D_{\nu} T_{A, n, e f g \rho}$ & 38 & 181 & $i \epsilon^{a b c} \bar{B}_{a}^{d} f_{+b}{ }^{e \mu \nu} h_{e}{ }^{f}{ }_{\mu}^{\lambda} D_{\nu} T_{A, n, c d f \lambda}$ & & 487 \\
\hline$i \epsilon^{a b c} \bar{B}_{a}{ }^{d} u_{b}{ }^{e \mu} u_{d}{ }^{f \nu} f_{-c}{ }^{g \lambda \rho} \sigma_{\mu \lambda} D_{\rho} T_{A, n, e f g \nu}$ & 39 & 182 & $i \epsilon^{a b c} \bar{B}_{a}^{d} f_{+b}{ }^{e \mu \nu} h_{e}^{f_{\mu}{ }^{\lambda} D_{\lambda} T_{A, n, c d f \nu}}$ & & 488 \\
\hline$i \epsilon^{a b c} \bar{B}_{a}{ }^{d} u_{b}{ }^{e \mu} u_{d}^{f \nu} f_{-e^{g \lambda \rho}} \sigma_{\mu \nu} D_{\lambda} T_{A, n, c f g \rho}$ & & 183 & $i \epsilon^{a b c} \bar{B}_{a}^{d} f_{+}{ }^{e f \mu \nu} h_{b e \mu}{ }^{\lambda} D_{\nu} T_{A, n, c d f \lambda}$ & & 489 \\
\hline$i \epsilon^{a b c} \bar{B}_{a}{ }^{d} u_{b}{ }^{e \mu} u_{d}{ }^{f \nu} f_{-e}{ }^{g \lambda \rho} \sigma_{\mu \lambda} D_{\nu} T_{A, n, c f g \rho}$ & & 184 & $i \epsilon^{a b c} \bar{B}_{a}{ }^{d} f_{+}{ }^{e f \mu \nu} h_{b e \mu}{ }^{\lambda} D_{\lambda} T_{A, n, c d f \nu}$ & & 490 \\
\hline$i \epsilon^{a b c} \bar{B}_{a}^{d} u_{b}^{e \mu} u_{d}^{f \nu} f_{-e^{g \lambda \rho}} \sigma_{\mu \lambda} D_{\rho} T_{A, n, c f g \nu}$ & & 185 & $i \epsilon^{a b c} \bar{B}_{a}{ }^{d} f_{+b}{ }^{e \mu \nu} h_{c}{ }^{f \lambda \rho} D_{\mu \lambda \rho} T_{A, n, d e f \nu}$ & 148 & 491 \\
\hline$i \epsilon^{a b c} \bar{B}_{a}^{d} u_{b}^{e \mu} u_{d}^{f \nu} f_{-e^{g \lambda \rho}} \sigma_{\nu \lambda} D_{\mu} T_{A, n, c f g \rho}$ & & 186 & $i \epsilon^{a b c} \bar{B}_{a}{ }^{d} f_{+b}{ }^{e \mu \nu} h_{d}{ }^{f \lambda \rho} D_{\mu \lambda \rho} T_{A, n, c e f \nu}$ & 149 & 492 \\
\hline$i \epsilon^{a b c} \bar{B}_{a}^{d} u_{b}{ }^{e \mu} u_{e}{ }^{f \nu} f_{-c}{ }^{g \lambda \rho} \sigma_{\mu \nu} D_{\lambda} T_{A, n, d f g \rho}$ & & 187 & $i \epsilon^{a b c} \bar{B}_{a}{ }^{d} f_{+b}{ }^{e \mu \nu} h_{e}{ }^{f \lambda \rho} D_{\mu \lambda \rho} T_{A, n, c d f \nu}$ & & 493 \\
\hline$i \epsilon^{a b c} \bar{B}_{a}{ }^{d} u_{b}{ }^{e \mu} u_{e}{ }^{f \nu} f_{-c}{ }^{g \lambda \rho} \sigma_{\mu \lambda} D_{\nu} T_{A, n, d f g \rho}$ & & 188 & $i \epsilon^{a b c} \bar{B}_{a}^{d} f_{+}{ }^{e f \mu \nu} h_{b e}{ }^{\lambda \rho} D_{\mu \lambda \rho} T_{A, n, c d f \nu}$ & & 494 \\
\hline$i \epsilon^{a b c} \bar{B}_{a}{ }^{d} u_{b}{ }^{e \mu} u_{e}{ }^{f \nu} f_{-c}{ }^{g \lambda \rho} \sigma_{\mu \lambda} D_{\rho} T_{A, n, d f g \nu}$ & & 189 & $\epsilon^{a b c} \bar{B}_{a}{ }^{d} f_{+b}{ }^{e \mu \nu} h_{c}{ }^{f \lambda \rho} \sigma_{\mu \nu} D_{\lambda} T_{A, n, d e f \rho}$ & 150 & 495 \\
\hline$i \epsilon^{a b c} \bar{B}_{a}^{d} u_{b}{ }^{e \mu} u_{e}{ }^{f \nu} f_{-c}{ }^{g \lambda \rho} \sigma_{\nu \lambda} D_{\mu} T_{A, n, d f g \rho}$ & & 190 & $\epsilon^{a b c} \bar{B}_{a}^{d} f_{+b}{ }^{e \mu \nu} h_{c}{ }^{f \lambda \rho} \sigma_{\mu \lambda} D_{\nu} T_{A, n, d e f \rho}$ & 151 & 496 \\
\hline$i \epsilon^{a b c} \bar{B}_{a}{ }^{d} u_{b}{ }^{e \mu} u_{e}{ }^{f \nu} f_{-d}{ }^{g \lambda \rho} \sigma_{\mu \nu} D_{\lambda} T_{A, n, c f g \rho}$ & & 191 & $\epsilon^{a b c} \bar{B}_{a}{ }^{d} f_{+b}{ }^{e \mu \nu} h_{d}{ }^{f \lambda \rho} \sigma_{\mu \nu} D_{\lambda} T_{A, n, c e f \rho}$ & 152 & 497 \\
\hline$i \epsilon^{a b c} \bar{B}_{a}{ }^{d} u_{b}{ }^{e \mu} u_{e}{ }^{f \nu} f_{-d^{g \lambda \rho}} \sigma_{\mu \lambda} D_{\nu} T_{A, n, c f g \rho}$ & & 192 & $\epsilon^{a b c} \bar{B}_{a}^{d} f_{+b}{ }^{e \mu \nu} h_{d}{ }^{f \lambda \rho} \sigma_{\mu \lambda} D_{\nu} T_{A, n, c e f \rho}$ & 153 & 498 \\
\hline$i \epsilon^{a b c} \bar{B}_{a}{ }^{d} u_{b}{ }^{e \mu} u_{e}{ }^{f \nu} f_{-d}{ }^{g \lambda \rho} \sigma_{\mu \lambda} D_{\rho} T_{A, n, c f g \nu}$ & & 193 & $\epsilon^{a b c} \bar{B}_{a}{ }^{d} f_{+b}{ }^{e \mu \nu} h_{e}{ }^{f \lambda \rho} \sigma_{\mu \nu} D_{\lambda} T_{A, n, c d f \rho}$ & & 499 \\
\hline$i \epsilon^{a b c} \bar{B}_{a}{ }^{d} u_{b}{ }^{e \mu} u_{e}{ }^{f \nu} f_{-d^{g \lambda \rho}} \sigma_{\nu \lambda} D_{\mu} T_{A, n, c f g \rho}$ & & 194 & $\epsilon^{a b c} \bar{B}_{a}^{d} f_{+b}{ }^{e \mu \nu} h_{e}{ }^{f \lambda \rho} \sigma_{\mu \lambda} D_{\nu} T_{A, n, c d f \rho}$ & & 500 \\
\hline$i \epsilon^{a b c} \bar{B}_{a}{ }^{d} u_{b}{ }^{e \mu} u_{e}{ }^{f \nu} f_{-f}{ }^{g \lambda \rho} \sigma_{\mu \nu} D_{\lambda} T_{A, n, c d g \rho}$ & & 195 & $\epsilon^{a b c} \bar{B}_{a}{ }^{d} f_{+}{ }^{e f \mu \nu} h_{b e}{ }^{\lambda \rho} \sigma_{\mu \nu} D_{\lambda} T_{A, n, c d f \rho}$ & & 501 \\
\hline$i \epsilon^{a b c} \bar{B}_{a}{ }^{d} u_{b}{ }^{e \mu} u_{e}{ }^{f \nu} f_{-f}^{g \lambda \rho} \sigma_{\mu \lambda} D_{\nu} T_{A, n, c d g \rho}$ & & 196 & $\epsilon^{a b c} \bar{B}_{a}^{d} f_{+}{ }^{e f \mu \nu} h_{b e}{ }^{\lambda \rho} \sigma_{\mu \lambda} D_{\nu} T_{A, n, c d f \rho}$ & & 502 \\
\hline$i \epsilon^{a b c} \bar{B}_{a}^{d} u_{b}^{e \mu} u_{e}^{f \nu} f_{-f}^{g \lambda \rho} \sigma_{\mu \lambda} D_{\rho} T_{A, n, c d g \nu}$ & & 197 & $i \epsilon^{a b c} \bar{B}_{a}{ }^{d} \nabla^{\mu} f_{+b}{ }^{e}{ }_{\mu}^{\nu} u_{c}{ }^{f \lambda} D_{\nu} T_{A, n, \text { def } \lambda}$ & 154 & 503 \\
\hline$i \epsilon^{a b c} \bar{B}_{a}^{d} u_{b}^{e \mu} u_{e}^{f \nu} f_{-f}^{g \lambda \rho} \sigma_{\nu \lambda} D_{\mu} T_{A, n, c d g \rho}$ & & 198 & $i \epsilon^{a b c} \bar{B}_{a}^{d} \nabla^{\mu} f_{+b}{ }^{e}{ }_{\mu}^{\nu} u_{c}{ }^{f \lambda} D_{\lambda} T_{A, n, d e f \nu}$ & 155 & 504 \\
\hline$i \epsilon^{a b c} \bar{B}_{a}^{d} u_{b}^{e \mu} u^{f g \nu} f_{-c f}^{\lambda \rho} \sigma_{\mu \nu} D_{\lambda} T_{A, n, \operatorname{deg} \rho}$ & & 199 & $i \epsilon^{a b c} \bar{B}_{a}{ }^{d} \nabla^{\mu} f_{+b}{ }^{e}{ }_{\mu}^{\nu} u_{d}^{f \lambda} D_{\nu} T_{A, n, c e f \lambda}$ & 156 & 505 \\
\hline$i \epsilon^{a b c} \bar{B}_{a}^{d} u_{b}^{e \mu} u^{f g \nu} f_{-c f}^{\lambda \rho} \sigma_{\mu \lambda} D_{\nu} T_{A, n, \operatorname{deg} \rho}$ & 40 & 200 & $i \epsilon^{a b c} \bar{B}_{a}^{d} \nabla^{\mu} f_{+b}{ }^{e}{ }_{\mu}^{\nu} u_{d}^{f \lambda} D_{\lambda} T_{A, n, c e f \nu}$ & 157 & 506 \\
\hline$i \epsilon^{a b c} \bar{B}_{a}{ }^{d} u_{b}{ }^{e \mu} u^{f g \nu} f_{-c f}^{\lambda \rho} \sigma_{\mu \lambda} D_{\rho} T_{A, n, \operatorname{deg} \nu}$ & 41 & 201 & $i \epsilon^{a b c} \bar{B}_{a}{ }^{d} \nabla^{\mu} f_{s,+\mu}{ }^{\nu} u_{b}{ }^{e \lambda} D_{\nu} T_{A, n, c d e \lambda}$ & 158 & \\
\hline$i \epsilon^{a b c} \bar{B}_{a}{ }^{d} u_{b}{ }^{e \mu} u^{f g \nu} f_{-c f}{ }^{\lambda \rho} \sigma_{\nu \lambda} D_{\mu} T_{A, n, \operatorname{deg} \rho}$ & & 202 & $i \epsilon^{a b c} \bar{B}_{a}{ }^{d} \nabla^{\mu} f_{s,+\mu}{ }^{\nu} u_{b}{ }^{e \lambda} D_{\lambda} T_{A, n, c d e \nu}$ & 159 & \\
\hline$i \epsilon^{a b c} \bar{B}_{a}{ }^{d} u_{b}{ }^{e \mu} u^{f g \nu} f_{-d f}{ }^{\lambda \rho} \sigma_{\mu \nu} D_{\lambda} T_{A, n, c e g \rho}$ & & 203 & $i \epsilon^{a b c} \bar{B}_{a}{ }^{d} \nabla^{\mu} f_{s,+}{ }^{\nu \lambda} u_{b}{ }^{e}{ }_{\mu} D_{\nu} T_{A, n, c d e \lambda}$ & 160 & \\
\hline$i \epsilon^{a b c} \bar{B}_{a}{ }^{d} u_{b}{ }^{e \mu} u^{f g \nu} f_{-d f}^{\lambda \rho} \sigma_{\mu \lambda} D_{\nu} T_{A, n, c e g \rho}$ & & 204 & $i \epsilon^{a b c} \bar{B}_{a}{ }^{d} \nabla^{\mu} f_{s,+}{ }^{\nu \lambda} u_{b}{ }^{e}{ }_{\nu} D_{\mu} T_{A, n, c d e \lambda}$ & 161 & \\
\hline$i \epsilon^{a b c} \bar{B}_{a}{ }^{d} u_{b}{ }^{e \mu} u^{f g \nu} f_{-d f}{ }^{\lambda \rho} \sigma_{\mu \lambda} D_{\rho} T_{A, n, c e g \nu}$ & & 205 & $i \epsilon^{a b c} \bar{B}_{a}{ }^{d} \nabla^{\mu} f_{s,+}{ }^{\nu \lambda} u_{b}{ }^{e \rho} D_{\mu \nu \rho} T_{A, n, c d e \lambda}$ & 162 & \\
\hline$i \epsilon^{a b c} \bar{B}_{a}{ }^{d} u_{b}{ }^{e \mu} u^{f g \nu} f_{-d f}{ }^{\lambda \rho} \sigma_{\nu \lambda} D_{\mu} T_{A, n, c e g \rho}$ & & 206 & $\epsilon^{a b c} \bar{B}_{a}{ }^{d} \nabla^{\mu} f_{s,+}{ }^{\nu \lambda} u_{b}{ }^{e \rho} \sigma_{\mu \nu} D_{\lambda} T_{A, n, c d e \rho}$ & 163 & \\
\hline$i \epsilon^{a b c} \bar{B}_{a}{ }^{d} u_{b}{ }^{e \mu} u^{f g \nu} f_{-e f}{ }^{\lambda \rho} \sigma_{\mu \nu} D_{\lambda} T_{A, n, c d g \rho}$ & & 207 & $\epsilon^{a b c} \bar{B}_{a}{ }^{d} \nabla^{\mu} f_{s,+}{ }^{\nu \lambda} u_{b}^{e \rho} \sigma_{\mu \nu} D_{\rho} T_{A, n, c d e \lambda}$ & 164 & \\
\hline$i \epsilon^{a b c} \bar{B}_{a}{ }^{d} u_{b}{ }^{e \mu} u^{f g \nu} f_{-e f}{ }^{\lambda \rho} \sigma_{\mu \lambda} D_{\nu} T_{A, n, c d g \rho}$ & & 208 & $\epsilon^{a b c} \bar{B}_{a}{ }^{d} \nabla^{\mu} f_{s,+}{ }^{\nu \lambda} u_{b}{ }^{e \rho} \sigma_{\mu \rho} D_{\nu} T_{A, n, c d e \lambda}$ & 165 & \\
\hline$i \epsilon^{a b c} \bar{B}_{a}{ }^{d} u_{b}{ }^{e \mu} u^{f g \nu} f_{-e f}^{\lambda \rho} \sigma_{\mu \lambda} D_{\rho} T_{A, n, c d g \nu}$ & & 209 & $i \epsilon^{a b c} \bar{B}_{a}{ }^{d} \nabla^{\mu} f_{+b}{ }^{e}{ }_{\mu}^{\nu} u_{e}{ }^{f \lambda} D_{\nu} T_{A, n, c d f \lambda}$ & & 507 \\
\hline
\end{tabular}


TABLE VII. (Continued)

\begin{tabular}{|c|c|c|c|c|c|}
\hline$P_{n}^{\left(N_{f}, 4\right)}$ & $S U(2)$ & $S U(3)$ & $P_{n}^{\left(N_{f}, 4\right)}$ & $S U(2)$ & $S U(3)$ \\
\hline$i \epsilon^{a b c} \bar{B}_{a}{ }^{d} u_{b}{ }^{e \mu} u^{f g \nu} f_{-e f}{ }^{\lambda \rho} \sigma_{\nu \lambda} D_{\mu} T_{A, n, c d g \rho}$ & & 210 & $i \epsilon^{a b c} \bar{B}_{a}{ }^{d} \nabla^{\mu} f_{+b}{ }^{e}{ }_{\mu}^{\nu} u_{e}{ }^{f \lambda} D_{\lambda} T_{A, n, c d f \nu}$ & & 508 \\
\hline$i \epsilon^{a b c} \bar{B}_{a}{ }^{d} u_{b}{ }^{e \mu} u^{f g \nu} f_{-g f}^{\lambda \rho} \sigma_{\mu \nu} D_{\lambda} T_{A, n, c d e \rho}$ & & 211 & $i \epsilon^{a b c} \bar{B}_{a}{ }^{d} \nabla^{\mu} f_{+}{ }^{e f}{ }_{\mu}^{\mu} u_{b e}{ }^{\lambda} D_{\nu} T_{A, n, c d f \lambda}$ & & 509 \\
\hline$i \epsilon^{a b c} \bar{B}_{a}{ }^{d} u_{b}{ }^{e \mu} u^{f g \nu} f_{-g f}^{\lambda \rho} \sigma_{\mu \lambda} D_{\nu} T_{A, n, c d e \rho}$ & & 212 & $i \epsilon^{a b c} \bar{B}_{a}{ }^{d} \nabla^{\mu} f_{+}{ }^{e f}{ }_{\mu}^{\mu} u_{b e}{ }^{\lambda} D_{\lambda} T_{A, n, c d f \nu}$ & & 510 \\
\hline$i \epsilon^{a b c} \bar{B}_{a}^{d} u_{b}^{e \mu} u^{f g \nu} f_{-g f}^{\lambda \rho} \sigma_{\mu \lambda} D_{\rho} T_{A, n, c d e \nu}$ & & 213 & $i \epsilon^{a b c} \bar{B}_{a}^{d} \nabla^{\mu} \nabla_{\mu} f_{+b}{ }^{e \nu \lambda} \gamma_{5} \gamma_{\nu} T_{A, n, c d e \lambda}$ & 166 & 511 \\
\hline$i \epsilon^{a b c} \bar{B}_{a}{ }^{d} u_{b}{ }^{e \mu} u^{f g \nu} f_{-g f}^{\lambda \rho} \sigma_{\nu \lambda} D_{\mu} T_{A, n, c d e \rho}$ & & 214 & $i \epsilon^{a b c} \bar{B}_{a}^{d} \nabla^{\mu} \nabla^{\nu} f_{+b}{ }^{e}{ }_{\mu}^{\lambda} \gamma_{5} \gamma_{\nu} T_{A, n, c d e \lambda}$ & 167 & 512 \\
\hline$\epsilon^{a b c} \bar{B}_{a}{ }^{d} u_{b}{ }^{e \mu} u_{c}{ }^{f}{ }_{\mu} h_{e}{ }^{g \nu \lambda} D_{\nu} T_{A, n, d f g \lambda}$ & 42 & 215 & $\epsilon^{a b c} \bar{B}_{a}{ }^{d} f_{+b}{ }^{e \mu \nu} f_{+c}{ }^{f}{ }_{\mu}^{\lambda} \gamma_{5} \gamma_{\nu} T_{A, n, d e f \lambda}$ & 168 & 513 \\
\hline$\epsilon^{a b c} \bar{B}_{a}{ }^{d} u_{b}{ }^{e \mu} u_{c}{ }^{f \nu} h_{d^{g}}^{g}{ }^{\lambda} D_{\nu} T_{A, n, e f g \lambda}$ & 43 & 216 & $\epsilon^{a b c} \bar{B}_{a}^{d} f_{+b}^{e \mu \nu} f_{+d^{f}{ }_{\mu} \lambda \gamma_{5} \gamma_{\nu} T_{A, n, c e f}}$ & 169 & 514 \\
\hline$\epsilon^{a b c} \bar{B}_{a}{ }^{d} u_{b}{ }^{e \mu} u_{c}{ }^{f \nu} h_{d}{ }^{g}{ }_{\mu}^{\lambda} D_{\lambda} T_{A, n, e f g \nu}$ & 44 & 217 & $\epsilon^{a b c} \bar{B}_{a}^{d} f_{+b}{ }^{e \mu \nu} f_{+e^{\prime}{ }_{\mu} \lambda \gamma_{5} \gamma_{\nu} T_{A, n, c d f \lambda}}$ & & 515 \\
\hline$\epsilon^{a b c} \bar{B}_{a}^{d} u_{b}{ }^{e \mu} u_{c}{ }^{f \nu} h_{e}^{g}{ }_{\mu}{ }^{\lambda} D_{\nu} T_{A, n, d f g \lambda}$ & 45 & 218 & $\epsilon^{a b c} \bar{B}_{a}^{d} f_{+b}{ }^{e \mu \nu} f_{+e^{f}{ }_{\mu}{ }^{\lambda} \gamma_{5} \gamma_{\lambda} T_{A, n, c d f \nu}}$ & & 516 \\
\hline$\epsilon^{a b c} \bar{B}_{a}{ }^{d} u_{b}{ }^{e \mu} u_{c}{ }^{f \nu} h_{e}{ }^{g}{ }_{\mu}{ }^{\lambda} D_{\lambda} T_{A, n, d f g \nu}$ & 46 & 219 & ${ }_{c}^{f \lambda \rho} \gamma_{5} \gamma_{\mu} D_{\nu \lambda} T_{A, n, \operatorname{def} \rho}$ & 170 & 517 \\
\hline$\epsilon^{a b c} \bar{B}_{a}^{d} u_{b}{ }^{e \mu} u_{c}{ }^{f \nu} h_{e}{ }^{g}{ }_{\nu}^{\lambda} D_{\mu} T_{A, n, d f g \lambda}$ & 47 & 220 & $\epsilon^{a b c} \bar{B}_{a}^{d} f_{+b}{ }^{e \mu \nu} f_{+d} f \lambda \rho \gamma_{5} \gamma_{\mu} D_{\nu \lambda} T_{A, n, c e f \rho}$ & 171 & 518 \\
\hline$\epsilon^{a b c} \bar{B}_{a}{ }^{d} u_{b}{ }^{e \mu} u_{c}{ }^{f \nu} h_{e}{ }^{g}{ }_{\nu}{ }^{\lambda} D_{\lambda} T_{A, n, d f g \mu}$ & & 221 & $\epsilon^{a b c} \bar{B}_{a}^{d} f_{+b}^{e \mu \nu} f_{s,+\mu}^{\lambda} \gamma_{5} \gamma_{\nu} T_{A, n, c d e \lambda}$ & 172 & \\
\hline$\epsilon^{a b c} \bar{B}_{a}{ }^{d} u_{b}{ }^{e \mu} u_{d}{ }^{f}{ }_{\mu} h_{c}{ }^{g \nu \lambda} D_{\nu} T_{A, n, e f g \lambda}$ & 48 & 222 & $\epsilon^{a b c} \bar{B}_{a}^{d} f_{+b}{ }^{e \mu \nu} f_{s,+\mu}{ }^{\lambda} \gamma_{5} \gamma_{\lambda} T_{A, n, c d e \nu}$ & 173 & \\
\hline$\epsilon^{a b c} \bar{B}_{a}{ }^{d} u_{b}{ }^{e \mu} u_{d}{ }^{f}{ }_{\mu} h_{e}{ }^{g \nu \lambda} D_{\nu} T_{A, n, c f g \lambda}$ & & 223 & $\epsilon^{a b c} \bar{B}_{a}^{d} f_{+b}{ }^{e \mu \nu} f_{s,+}{ }^{\lambda \rho} \gamma_{5} \gamma_{\mu} D_{\nu \lambda} T_{A, n, c d e \rho}$ & 174 & \\
\hline$\epsilon^{a b c} \bar{B}_{a}{ }^{d} u_{b}{ }^{e \mu} u_{d}{ }^{f \nu} h_{e}{ }^{g}{ }_{\mu}{ }^{\lambda} D_{\nu} T_{A, n, c f g \lambda}$ & & 224 & $\epsilon^{a b c} \bar{B}_{a}^{d} f_{+b}{ }^{e \mu \nu} f_{s,+}^{\lambda \rho} \gamma_{5} \gamma_{\lambda} D_{\mu \rho} T_{A, n, c d e \nu}$ & 175 & \\
\hline$\epsilon^{a b c} \bar{B}_{a}{ }^{d} u_{b}{ }^{e \mu} u_{d}{ }^{f \nu} h_{e}{ }_{\mu}^{g}{ }^{\lambda} D_{\lambda} T_{A, n, c f g \nu}$ & & 225 & $\epsilon^{a b c} \bar{B}_{a}^{d} f_{+b}{ }^{e \mu \nu} f_{+e}{ }^{f \lambda \rho} \gamma_{5} \gamma_{\mu} D_{\nu \lambda} T_{A, n, c d f \rho}$ & & 519 \\
\hline$\epsilon^{a b c} \bar{B}_{a}{ }^{d} u_{b}{ }^{e \mu} u_{d}{ }^{f \nu} h_{e}{ }^{g}{ }_{\nu}^{\lambda} D_{\mu} T_{A, n, c f g \lambda}$ & & 226 & 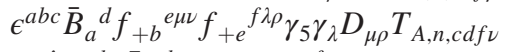 & & 520 \\
\hline$\epsilon^{a b c} \bar{B}_{a}{ }^{d} u_{b}{ }^{e \mu} u_{d}{ }^{f \nu} h_{e}{ }^{g}{ }_{\nu}{ }^{\lambda} D_{\lambda} T_{A, n, c f g \mu}$ & & 227 & $\varepsilon^{\mu \nu \lambda \rho} \epsilon^{a b c} \bar{B}_{a}{ }^{d} f_{+b}{ }^{e}{ }_{\mu \nu} f_{+c}{ }_{\lambda}^{f}{ }_{\lambda}^{\sigma} D_{\rho} T_{A, n, \text { def } \sigma}$ & 176 & 521 \\
\hline$\epsilon^{a b c} \bar{B}_{a}{ }^{d} u_{b}{ }^{e \mu} u_{e}{ }^{f}{ }_{\mu} h_{c}{ }^{g \nu \lambda} D_{\nu} T_{A, n, d f g \lambda}$ & & 228 & $\varepsilon^{\mu \nu \lambda \rho} \epsilon^{a b c} \bar{B}_{a}^{d} f_{+b}{ }^{e}{ }_{\mu \nu} f_{s,+\lambda}{ }^{\sigma} D_{\rho} T_{A, n, c d e \sigma}$ & 177 & \\
\hline$\epsilon^{a b c} \bar{B}_{a}{ }^{d} u_{b}{ }^{e \mu} u_{e}{ }^{f}{ }_{\mu} h_{d}{ }^{g \nu \lambda} D_{\nu} T_{A, n, c f g \lambda}$ & & 229 & $\varepsilon^{\mu \nu \lambda \rho} \epsilon^{a b c} \bar{B}_{a}{ }^{d} f_{+b}{ }^{e}{ }_{\mu \nu} f_{+e^{f}{ }_{\lambda}{ }^{\sigma} D_{\rho} T_{A, n, c d f \sigma}}$ & & 522 \\
\hline$\epsilon^{a b c} \bar{B}_{a}{ }^{d} u_{b}{ }^{e \mu} u_{e}{ }^{f}{ }_{\mu} h_{f}^{g \nu \lambda} D_{\nu} T_{A, n, c d g \lambda}$ & & 230 & $\epsilon^{a b c} \bar{B}_{a}{ }^{d} u_{b}{ }^{e \mu} u_{c}{ }^{f \nu} \chi_{+d}{ }^{g} \gamma_{5} \gamma_{\mu} T_{A, n, e f g \nu}$ & 178 & 523 \\
\hline$\epsilon^{a b c} \bar{B}_{a}{ }^{d} u_{b}{ }^{e \mu} u_{e}{ }^{f \nu} h_{c}{ }^{g}{ }_{\mu}{ }^{\lambda} D_{\nu} T_{A, n, d f g \lambda}$ & & 231 & $\epsilon^{a b c} \bar{B}_{a}{ }^{d} u_{b}{ }^{e \mu} u_{c}{ }^{f \nu} \chi_{+e}{ }^{g} \gamma_{5} \gamma_{\mu} T_{A, n, d f g \nu}$ & 179 & 524 \\
\hline$\epsilon^{a b c} \bar{B}_{a}{ }^{d} u_{b}{ }^{e \mu} u_{e}{ }^{f \nu} h_{c}{ }^{g}{ }^{\lambda} D_{\lambda} T_{A, n, d f g \nu}$ & & 232 & $\epsilon^{a b c} \bar{B}_{a}{ }^{d} u_{b}{ }^{e \mu} u_{c}{ }^{f \nu} \chi_{+e}{ }^{g} \gamma_{5} \gamma_{\nu} T_{A, n, d f g \mu}$ & 180 & 525 \\
\hline$\epsilon^{a b c} \bar{B}_{a}{ }^{d} u_{b}{ }^{e \mu} u_{e}{ }^{f \nu} h_{c}{ }^{g}{ }_{\nu}{ }^{\lambda} D_{\mu} T_{A, n, d f g \lambda}$ & & 233 & $\epsilon^{a b c} \bar{B}_{a}^{d} u_{b}{ }^{e \mu} u_{d}{ }^{f \nu} \chi_{+c}{ }^{g} \gamma_{5} \gamma_{\mu} T_{A, n, e f g \nu}$ & 181 & 526 \\
\hline$\epsilon^{a b c} \bar{B}_{a}{ }^{d} u_{b}{ }^{e \mu} u_{e}{ }^{f \nu} h_{d}^{g}{ }_{\mu}^{\lambda} D_{\nu} T_{A, n, c f g \lambda}$ & & 234 & $\epsilon^{a b c} \bar{B}_{a}{ }^{d} u_{b}{ }^{e \mu} u_{d}{ }^{f \nu} \chi_{+e}{ }^{g} \gamma_{5} \gamma_{\mu} T_{A, n, c f g \nu}$ & & 527 \\
\hline$\epsilon^{a b c} \bar{B}_{a}{ }^{d} u_{b}{ }^{e \mu} u_{e}{ }^{f \nu} h_{d}{ }^{g}{ }_{\mu}{ }^{\lambda} D_{\lambda} T_{A, n, c f g \nu}$ & & 235 & $\epsilon^{a b c} \bar{B}_{a}^{d} u_{b}{ }^{e \mu} u_{d}{ }^{f \nu} \chi_{+e^{g} \gamma_{5} \gamma_{\nu} T_{A, n, c f g \mu}}$ & & 528 \\
\hline$\epsilon^{a b c} \bar{B}_{a}{ }^{d} u_{b}{ }^{e \mu} u_{e} e^{f \nu} h_{d}{ }^{g}{ }_{\nu}^{\lambda} D_{\mu} T_{A, n, c f g \lambda}$ & & 236 & $\epsilon^{a b c} \bar{B}_{a}{ }^{d} u_{b}{ }^{e \mu} u_{e}{ }^{f \nu} \chi_{+c}{ }^{g} \gamma_{5} \gamma_{\mu} T_{A, n, d f g \nu}$ & & 529 \\
\hline$\epsilon^{a b c} \bar{B}_{a}{ }^{d} u_{b}{ }^{e \mu} u_{e}{ }^{f \nu} h_{f^{\prime}}^{g}{ }_{\mu}^{\lambda} D_{\nu} T_{A, n, c d g \lambda}$ & & 237 & $\epsilon^{a b c} \bar{B}_{a}{ }^{d} u_{b}{ }^{e \mu} u_{e}{ }^{f \nu} \chi_{+c}{ }^{g} \gamma_{5} \gamma_{\nu} T_{A, n, d f g \mu}$ & & 530 \\
\hline$\epsilon^{a b c} \bar{B}_{a}{ }^{d} u_{b}{ }^{e \mu} u_{e}{ }^{f \nu} h_{f^{g}}^{g}{ }^{\lambda} D_{\lambda} T_{A, n, c d g \nu}$ & & 238 & $\epsilon^{a b c} \bar{B}_{a}^{d} u_{b}{ }^{e \mu} u_{e}{ }^{f \nu} \chi_{+d^{g}} \gamma_{5} \gamma_{\mu} T_{A, n, c f g \nu}$ & & 531 \\
\hline$\epsilon^{a b c} \bar{B}_{a}{ }^{d} u_{b}{ }^{e \mu} u_{e}{ }^{f \nu} h_{f^{g}}{ }_{\nu} \lambda D_{\mu} T_{A, n, c d g \lambda}$ & & 239 & $\epsilon^{a b c} \bar{B}_{a}^{d} u_{b}{ }^{e \mu} u_{e}{ }^{f \nu} \chi_{+d^{g}} \gamma_{5} \gamma_{\nu} T_{A, n, c f g \mu}$ & & 532 \\
\hline$\epsilon^{a b c} \bar{B}_{a}{ }^{d} u_{b}{ }^{e \mu} u_{e}{ }^{f \nu} h_{f}^{g}{ }_{\nu}{ }^{\lambda} D_{\lambda} T_{A, n, c d g \mu}$ & & 240 & $\epsilon^{a b c} \bar{B}_{a}^{d} u_{b}{ }^{e \mu} u_{e}{ }^{f \nu} \chi_{+f}{ }^{g} \gamma_{5} \gamma_{\mu} T_{A, n, c d g \nu}$ & & 533 \\
\hline$\epsilon^{a b c} \bar{B}_{a}{ }^{d} u_{b}{ }^{e \mu} u^{f g}{ }_{\mu} h_{e f}{ }^{\nu \lambda} D_{\nu} T_{A, n, c d g \lambda}$ & & 241 & $\epsilon^{a b c} \bar{B}_{a}^{d} u_{b}{ }^{e \mu} u_{e}{ }^{f \nu} \chi_{+f^{g}} \gamma_{5} \gamma_{\nu} T_{A, n, c d g \mu}$ & & 534 \\
\hline$\epsilon^{a b c} \bar{B}_{a}{ }^{d} u_{b}{ }^{e \mu} u^{f g}{ }_{\mu} h_{g f}{ }^{\nu \lambda} D_{\nu} T_{A, n, c d e \lambda}$ & & 242 & $\epsilon^{a b c} \bar{B}_{a}^{d} u_{b}{ }^{e \mu} u^{f g \nu} \chi_{+c f} \gamma_{5} \gamma_{\mu} T_{A, n, \operatorname{deg} \nu}$ & 182 & 535 \\
\hline$\epsilon^{a b c} \bar{B}_{a}{ }^{d} u_{b}{ }^{e \mu} u^{f g \nu} h_{e f \mu}{ }^{\lambda} D_{\nu} T_{A, n, c d g \lambda}$ & & 243 & $\epsilon^{a b c} \bar{B}_{a}{ }^{d} u_{b}{ }^{e \mu} u^{f g \nu} \chi_{+c f} \gamma_{5} \gamma_{\nu} T_{A, n, \operatorname{deg} \mu}$ & & 536 \\
\hline$\epsilon^{a b c} \bar{B}_{a}{ }^{d} u_{b}{ }^{e \mu} u^{f g \nu} h_{e f \mu}{ }^{\lambda} D_{\lambda} T_{A, n, c d g \nu}$ & & 244 & $\epsilon^{a b c} \bar{B}_{a}{ }^{d} u_{b}{ }^{e \mu} u^{f g \nu} \chi_{+d f} \gamma_{5} \gamma_{\mu} T_{A, n, c e g \nu}$ & & 537 \\
\hline$\epsilon^{a b c} \bar{B}_{a}^{d} u_{b}{ }^{e \mu} u_{c}{ }^{f \nu} h_{d}^{g \lambda \rho} D_{\mu \lambda \rho} T_{A, n, e f g \nu}$ & 49 & 245 & $\epsilon^{a b c} \bar{B}_{a}{ }^{d} u_{b}{ }^{e \mu} u^{f g \nu} \chi_{+d f} \gamma_{5} \gamma_{\nu} T_{A, n, c e g \mu}$ & & 538 \\
\hline$\epsilon^{a b c} \bar{B}_{a}{ }^{d} u_{b}{ }^{e \mu} u_{c}{ }^{f \nu} h_{e}{ }^{g \lambda \rho} D_{\mu \nu \lambda} T_{A, n, d f g \rho}$ & 50 & 246 & $\epsilon^{a b c} \bar{B}_{a}{ }^{d} u_{b}{ }^{e \mu} u^{f g \nu} \chi_{+e f} \gamma_{5} \gamma_{\mu} T_{A, n, c d g \nu}$ & & 539 \\
\hline$\epsilon^{a b c} \bar{B}_{a}^{d} u_{b}{ }^{e \mu} u_{c}{ }^{f \nu} h_{e}{ }^{g \lambda \rho} D_{\mu \lambda \rho} T_{A, n, d f g \nu}$ & 51 & 247 & $\epsilon^{a b c} \bar{B}_{a}{ }^{d} u_{b}{ }^{e \mu} u^{f g \nu} \chi_{+e f} \gamma_{5} \gamma_{\nu} T_{A, n, c d g \mu}$ & & 540 \\
\hline$\epsilon^{a b c} \bar{B}_{a}^{d} u_{b}{ }^{e \mu} u_{c}{ }^{f \nu} h_{e}{ }^{g \lambda \rho} D_{\nu \lambda \rho} T_{A, n, d f g \mu}$ & & 248 & $\epsilon^{a b c} \bar{B}_{a}{ }^{d} u_{b}{ }^{e \mu} u^{f g \nu} \chi_{+g f} \gamma_{5} \gamma_{\mu} T_{A, n, c d e \nu}$ & & 541 \\
\hline$\epsilon^{a b c} \bar{B}_{a}{ }^{d} u_{b}{ }^{e \mu} u_{d}^{f \nu} h_{c}^{g \lambda \rho} D_{\mu \nu \lambda} T_{A, n, e f g \rho}$ & 52 & 249 & $\epsilon^{a b c} \bar{B}_{a}{ }^{d} u_{b}{ }^{e \mu} u^{f g \nu} \chi_{+g f} \gamma_{5} \gamma_{\nu} T_{A, n, c d e \mu}$ & & 542 \\
\hline$\epsilon^{a b c} \bar{B}_{a}^{d} u_{b}{ }^{e \mu} u_{d}{ }^{f \nu} h_{e}{ }^{g \lambda \rho} D_{\mu \nu \lambda} T_{A, n, c f g \rho}$ & & 250 & $\epsilon^{a b c} \bar{B}_{a}{ }^{d} u_{b}{ }^{e \mu} u_{c}{ }^{f \nu} \chi_{+, s} \gamma_{5} \gamma_{\mu} T_{A, n, \text { def } \nu}$ & 183 & 543 \\
\hline$\epsilon^{a b c} \bar{B}_{a}^{d} u_{b}{ }^{e \mu} u_{d}{ }^{f \nu} h_{e}{ }^{g \lambda \rho} D_{\mu \lambda \rho} T_{A, n, c f g \nu}$ & & 251 & $\epsilon^{a b c} \bar{B}_{a}^{d} u_{b}{ }^{e \mu} u_{d}{ }^{f \nu} \chi_{+, s} \gamma_{5} \gamma_{\mu} T_{A, n, c e f \nu}$ & 184 & 544 \\
\hline$\epsilon^{a b c} \bar{B}_{a}{ }^{d} u_{b}{ }^{e \mu} u_{d}{ }^{f \nu} h_{e}{ }^{g \lambda \rho} D_{\nu \lambda \rho} T_{A, n, c f g \mu}$ & & 252 & $\epsilon^{a b c} \bar{B}_{a}{ }^{d} u_{b}{ }^{e \mu} u_{e}{ }^{f \nu} \chi_{+, s} \gamma_{5} \gamma_{\mu} T_{A, n, c d f \nu}$ & & 545 \\
\hline$\epsilon^{a b c} \bar{B}_{a}{ }^{d} u_{b}{ }^{e \mu} u_{e}{ }^{f \nu} h_{c}{ }^{g \lambda \rho} D_{\mu \nu \lambda} T_{A, n, d f g \rho}$ & & 253 & $\epsilon^{a b c} \bar{B}_{a}{ }^{d} u_{b}{ }^{e \mu} u_{e} e^{f \nu} \chi_{+, s} \gamma_{5} \gamma_{\nu} T_{A, n, c d f \mu}$ & & 546 \\
\hline$\epsilon^{a b c} \bar{B}_{a}{ }^{d} u_{b}{ }^{e \mu} u_{e}{ }^{f \nu} h_{c}{ }^{g \lambda \rho} D_{\mu \lambda \rho} T_{A, n, d f g \nu}$ & & 254 & $\epsilon^{a b c} \bar{B}_{a}^{d} f_{-b}^{e \mu \nu} \chi_{+c}^{f} D_{\mu} T_{A, n, d e f \nu}$ & 185 & 547 \\
\hline$\epsilon^{a b c} \bar{B}_{a}{ }^{d} u_{b}{ }^{e \mu} u_{e}{ }^{f \nu} h_{d}{ }^{g \lambda \rho} D_{\mu \nu \lambda} T_{A, n, c f g \rho}$ & & 255 & $\epsilon^{a b c} \bar{B}_{a}^{d} f_{-b}^{e \mu \nu} \chi_{+d}{ }^{f} D_{\mu} T_{A, n, c e f \nu}$ & 186 & 548 \\
\hline$\epsilon^{a b c} \bar{B}_{a}^{d} u_{b}{ }^{e \mu} u_{e}{ }^{f \nu} h_{d}{ }^{g \lambda \rho} D_{\mu \lambda \rho} T_{A, n, c f g \nu}$ & & 256 & $\epsilon^{a b c} \bar{B}_{a}^{d} f_{-b}{ }^{e \mu \nu} \chi_{+e^{f}} D_{\mu} T_{A, n, c d f \nu}$ & & 549 \\
\hline$\epsilon^{a b c} \bar{B}_{a}^{d} u_{b}^{e \mu} u_{e}^{f \nu} h_{f}^{g \lambda \rho} D_{\mu \nu \lambda} T_{A, n, c d g \rho}$ & & 257 & $\epsilon^{a b c} \bar{B}_{a}{ }^{d} f_{-}{ }^{e f \mu \nu} \chi_{+b e} D_{\mu} T_{A, n, c d f \nu}$ & & 550 \\
\hline
\end{tabular}


TABLE VII. (Continued)

\begin{tabular}{|c|c|c|c|c|c|}
\hline$P_{n}^{\left(N_{f}, 4\right)}$ & $S U(2)$ & $S U(3)$ & $P_{n}^{\left(N_{f}, 4\right)}$ & $S U(2)$ & $S U(3)$ \\
\hline$\epsilon^{a b c} \bar{B}_{a}{ }^{d} u_{b}{ }^{e \mu} u_{e}{ }^{f \nu} h_{f}^{g \lambda \rho} D_{\mu \lambda \rho} T_{A, n, c d g \nu}$ & & 258 & $\epsilon^{a b c} \bar{B}_{a}{ }^{d} h_{b}{ }^{e \mu \nu} \chi_{+c}{ }^{f} D_{\mu} T_{A, n, \text { def } \nu}$ & 187 & 551 \\
\hline$\epsilon^{a b c} \bar{B}_{a}{ }^{d} u_{b}{ }^{e \mu} u_{e}{ }^{f \nu} h_{f}{ }^{g \lambda \rho} D_{\nu \lambda \rho} T_{A, n, c d g \mu}$ & & 259 & $\epsilon^{a b c} \bar{B}_{a}{ }^{d} h_{b}{ }^{e \mu \nu} \chi_{+d}{ }^{f} D_{\mu} T_{A, n, c e f \nu}$ & 188 & 552 \\
\hline$\epsilon^{a b c} \bar{B}_{a}{ }^{d} u_{b}{ }^{e \mu} u^{f g \nu} h_{e f}{ }^{\lambda \rho} D_{\mu \nu \lambda} T_{A, n, c d g \rho}$ & & 260 & $\epsilon^{a b c} \bar{B}_{a}^{d} h_{b}{ }^{e \mu \nu} \chi_{+e}{ }^{f} D_{\mu} T_{A, n, c d f \nu}$ & & 553 \\
\hline$\epsilon^{a b c} \bar{B}_{a}{ }^{d} u_{b}{ }^{e \mu} u^{f g \nu} h_{e f}{ }^{\lambda \rho} D_{\mu \lambda \rho} T_{A, n, c d g \nu}$ & & 261 & $\epsilon^{a b c} \bar{B}_{a}{ }^{d} h^{e f \mu \nu} \chi_{+b e} D_{\mu} T_{A, n, c d f \nu}$ & & 554 \\
\hline$i \epsilon^{a b c} \bar{B}_{a}{ }^{d} u_{b}{ }^{e \mu} u_{c}{ }^{f \nu} h_{d}{ }^{g \lambda \rho} \sigma_{\mu \nu} D_{\lambda} T_{A, n, e f g \rho}$ & 53 & 262 & $\epsilon^{a b c} \bar{B}_{a}^{d} u_{b}{ }^{e \mu} \nabla^{\nu} \chi_{+, s} D_{\mu} T_{A, n, c d e \nu}$ & 189 & 555 \\
\hline$i \epsilon^{a b c} \bar{B}_{a}{ }^{d} u_{b}{ }^{e \mu} u_{c}{ }^{f \nu} h_{d}{ }^{g \lambda \rho} \sigma_{\mu \lambda} D_{\nu} T_{A, n, e f g \rho}$ & 54 & 263 & $\epsilon^{a b c} \bar{B}_{a}^{d} u_{b}^{e \mu} \nabla^{\nu} \chi_{+, s} D_{\nu} T_{A, n, c d e \mu}$ & 190 & 556 \\
\hline$i \epsilon^{a b c} \bar{B}_{a}{ }^{d} u_{b}{ }^{e \mu} u_{c}{ }^{f \nu} h_{e}{ }^{g \lambda \rho} \sigma_{\mu \nu} D_{\lambda} T_{A, n, d f g \rho}$ & 55 & 264 & $\epsilon^{a b c} \bar{B}_{a}{ }^{d} \nabla^{\mu} \nabla^{\nu} \chi_{+b}{ }^{e} \gamma_{5} \gamma_{\mu} T_{A, n, c d e \nu}$ & 191 & 557 \\
\hline$i \epsilon^{a b c} \bar{B}_{a}{ }^{d} u_{b}{ }^{e \mu} u_{c}{ }^{f \nu} h_{e}{ }^{g \lambda \rho} \sigma_{\mu \lambda} D_{\nu} T_{A, n, d f g \rho}$ & 56 & 265 & $i \epsilon^{a b c} \bar{B}_{a}^{d} f_{+b}{ }^{e \mu \nu} \chi_{+c}{ }^{f} \gamma_{5} \gamma_{\mu} T_{A, n, \text { def }}$ & 192 & 558 \\
\hline$i \epsilon^{a b c} \bar{B}_{a}{ }^{d} u_{b}{ }^{e \mu} u_{c}{ }^{f \nu} h_{e}{ }^{g \lambda \rho} \sigma_{\mu \lambda} D_{\rho} T_{A, n, d f g \nu}$ & 57 & 266 & $i \epsilon^{a b c} \bar{B}_{a}^{d} f_{+b}{ }^{e \mu \nu} \chi_{+d}{ }^{f} \gamma_{5} \gamma_{\mu} T_{A, n, c e f \nu}$ & 193 & 559 \\
\hline$i \epsilon^{a b c} \bar{B}_{a}{ }^{d} u_{b}{ }^{e \mu} u_{c}{ }^{f \nu} h_{e}{ }^{g \lambda \rho} \sigma_{\nu \lambda} D_{\mu} T_{A, n, d f g \rho}$ & 58 & 267 & $i \epsilon^{a b c} \bar{B}_{a}^{d} f_{s,+}^{\mu \nu} \chi_{+b}{ }^{e} \gamma_{5} \gamma_{\mu} T_{A, n, c d e \nu}$ & 194 & \\
\hline$i \epsilon^{a b c} \bar{B}_{a}{ }^{d} u_{b}{ }^{e \mu} u_{d}{ }^{f \nu} h_{e}{ }^{g \lambda \rho} \sigma_{\mu \nu} D_{\lambda} T_{A, n, c f g \rho}$ & & 268 & $i \epsilon^{a b c} \bar{B}_{a}^{d} f_{+b}{ }^{e \mu \nu} \chi_{+e^{f} \gamma_{5} \gamma_{\mu} T_{A, n, c d f \nu}}$ & & 560 \\
\hline$i \epsilon^{a b c} \bar{B}_{a}{ }^{d} u_{b}{ }^{e \mu} u_{d}{ }^{f \nu} h_{e}{ }^{g \lambda \rho} \sigma_{\mu \lambda} D_{\nu} T_{A, n, c f g \rho}$ & & 269 & $i \epsilon^{a b c} \bar{B}_{a}{ }^{d} f_{+}{ }^{e f \mu \nu} \chi_{+b e} \gamma_{5} \gamma_{\mu} T_{A, n, c d f \nu}$ & & 561 \\
\hline$i \epsilon^{a b c} \bar{B}_{a}{ }^{d} u_{b}{ }^{e \mu} u_{d}{ }^{f \nu} h_{e}{ }^{g \lambda \rho} \sigma_{\mu \lambda} D_{\rho} T_{A, n, c f g \nu}$ & & 270 & $i \epsilon^{a b c} \bar{B}_{a}^{d} f_{+b}^{e \mu \nu} \chi_{+, s} \gamma_{5} \gamma_{\mu} T_{A, n, c d e \nu}$ & 195 & 562 \\
\hline$i \epsilon^{a b c} \bar{B}_{a}{ }^{d} u_{b}{ }^{e \mu} u_{d}{ }^{f \nu} h_{e}{ }^{g \lambda \rho} \sigma_{\nu \lambda} D_{\mu} T_{A, n, c f g \rho}$ & & 271 & $i \epsilon^{a b c} \bar{B}_{a}{ }^{d} u_{b}{ }^{e \mu} u_{c}{ }^{f \nu} \chi_{-d}{ }^{g} D_{\mu} T_{A, n, e f g \nu}$ & 196 & 563 \\
\hline$i \epsilon^{a b c} \bar{B}_{a}{ }^{d} u_{b}{ }^{e \mu} u_{e}{ }^{f \nu} h_{c}{ }^{g \lambda \rho} \sigma_{\mu \nu} D_{\lambda} T_{A, n, d f g \rho}$ & & 272 & $i \epsilon^{a b c} \bar{B}_{a}{ }^{d} u_{b}{ }^{e \mu} u_{c}{ }^{f \nu} \chi_{-e}{ }^{g} D_{\mu} T_{A, n, d f g \nu}$ & 197 & 564 \\
\hline$i \epsilon^{a b c} \bar{B}_{a}{ }^{d} u_{b}{ }^{e \mu} u_{e}{ }^{f \nu} h_{c}{ }^{g \lambda \rho} \sigma_{\mu \lambda} D_{\nu} T_{A, n, d f g \rho}$ & & 273 & $i \epsilon^{a b c} \bar{B}_{a}{ }^{d} u_{b}{ }^{e \mu} u_{c}{ }^{f \nu} \chi_{-e}{ }^{g} D_{\nu} T_{A, n, d f g \mu}$ & 198 & 565 \\
\hline$i \epsilon^{a b c} \bar{B}_{a}{ }^{d} u_{b}{ }^{e \mu} u_{e}{ }^{f \nu} h_{c}{ }^{g \lambda \rho} \sigma_{\mu \lambda} D_{\rho} T_{A, n, d f g \nu}$ & & 274 & $i \epsilon^{a b c} \bar{B}_{a}{ }^{d} u_{b}{ }^{e \mu} u_{d}{ }^{f \nu} \chi_{-c}{ }^{g} D_{\mu} T_{A, n, e f g \nu}$ & 199 & 566 \\
\hline$i \epsilon^{a b c} \bar{B}_{a}{ }^{d} u_{b}{ }^{e \mu} u_{e}{ }^{f \nu} h_{d}{ }^{g \lambda \rho} \sigma_{\mu \nu} D_{\lambda} T_{A, n, c f g \rho}$ & & 275 & $i \epsilon^{a b c} \bar{B}_{a}{ }^{d} u_{b}{ }^{e \mu} u_{d}{ }^{f \nu} \chi_{-e}{ }^{g} D_{\mu} T_{A, n, c f g \nu}$ & & 567 \\
\hline$i \epsilon^{a b c} \bar{B}_{a}{ }^{d} u_{b}{ }^{e \mu} u_{e}{ }^{f \nu} h_{d}{ }^{g \lambda \rho} \sigma_{\mu \lambda} D_{\nu} T_{A, n, c f g \rho}$ & & 276 & $i \epsilon^{a b c} \bar{B}_{a}{ }^{d} u_{b}{ }^{e \mu} u_{d}{ }^{f \nu} \chi_{-e}{ }^{g} D_{\nu} T_{A, n, c f g \mu}$ & & 568 \\
\hline$i \epsilon^{a b c} \bar{B}_{a}{ }^{d} u_{b}{ }^{e \mu} u_{e} e^{f \nu} h_{d}{ }^{g \lambda \rho} \sigma_{\mu \lambda} D_{\rho} T_{A, n, c f g \nu}$ & & 277 & $i \epsilon^{a b c} \bar{B}_{a}{ }^{d} u_{b}{ }^{e \mu} u_{e}{ }^{f \nu} \chi_{-c}{ }^{g} D_{\mu} T_{A, n, d f g \nu}$ & & 569 \\
\hline$i \epsilon^{a b c} \bar{B}_{a}{ }^{d} u_{b}{ }^{e \mu} u_{e}{ }^{f \nu} h_{f}{ }^{g \lambda \rho} \sigma_{\mu \nu} D_{\lambda} T_{A, n, c d g \rho}$ & & 278 & $i \epsilon^{a b c} \bar{B}_{a}{ }^{d} u_{b}{ }^{e \mu} u_{e}{ }^{f \nu} \chi_{-c}{ }^{g} D_{\nu} T_{A, n, d f g \mu}$ & & 570 \\
\hline$i \epsilon^{a b c} \bar{B}_{a}{ }^{d} u_{b}{ }^{e \mu} u_{e}{ }^{f \nu} h_{f}{ }^{g \lambda \rho} \sigma_{\mu \lambda} D_{\nu} T_{A, n, c d g \rho}$ & & 279 & $i \epsilon^{a b c} \bar{B}_{a}{ }^{d} u_{b}{ }^{e \mu} u_{e}{ }^{f \nu} \chi_{-d}{ }^{g} D_{\mu} T_{A, n, c f g \nu}$ & & 571 \\
\hline$i \epsilon^{a b c} \bar{B}_{a}{ }^{d} u_{b}{ }^{e \mu} u_{e}{ }^{f \nu} h_{f}{ }^{g \lambda \rho} \sigma_{\mu \lambda} D_{\rho} T_{A, n, c d g \nu}$ & & 280 & $i \epsilon^{a b c} \bar{B}_{a}{ }^{d} u_{b}{ }^{e \mu} u_{e}{ }^{f \nu} \chi_{-d^{g}} D_{\nu} T_{A, n, c f g \mu}$ & & 572 \\
\hline$i \epsilon^{a b c} \bar{B}_{a}{ }^{d} u_{b}{ }^{e \mu} u_{e}{ }^{f \nu} h_{f}{ }^{g \lambda \rho} \sigma_{\nu \lambda} D_{\mu} T_{A, n, c d g \rho}$ & & 281 & $i \epsilon^{a b c} \bar{B}_{a}{ }^{d} u_{b}{ }^{e \mu} u_{e}{ }^{f \nu} \chi_{-f}{ }^{g} D_{\mu} T_{A, n, c d g \nu}$ & & 573 \\
\hline$i \epsilon^{a b c} \bar{B}_{a}{ }^{d} u_{b}{ }^{e \mu} u^{f g \nu} h_{e f}{ }^{\lambda \rho} \sigma_{\mu \nu} D_{\lambda} T_{A, n, c d g \rho}$ & & 282 & $i \epsilon^{a b c} \bar{B}_{a}{ }^{d} u_{b}{ }^{e \mu} u_{e}{ }^{f \nu} \chi_{-f^{g}} D_{\nu} T_{A, n, c d g \mu}$ & & 574 \\
\hline$f^{\lambda \rho} \sigma_{\mu \lambda} D_{\nu} T_{A, n, c d g \rho}$ & & 283 & $i \epsilon^{a b c} \bar{B}_{a}{ }^{d} u_{b}{ }^{e \mu} u^{f g \nu} \chi_{-c f} D_{\mu} T_{A, n, \operatorname{deg} \nu}$ & 200 & 575 \\
\hline$i \epsilon^{a b c} \bar{B}_{a}{ }^{d} u_{b}{ }^{e \mu} u^{f g \nu} h_{e f}{ }^{\lambda \rho} \sigma_{\mu \lambda} D_{\rho} T_{A, n, c d g \nu}$ & & 284 & $i \epsilon^{a b c} \bar{B}_{a}{ }^{d} u_{b}{ }^{e \mu} u^{f g \nu} \chi_{-c f} D_{\nu} T_{A, n, \operatorname{deg} \mu}$ & & 576 \\
\hline$\epsilon^{a b c} \bar{B}_{a}^{d} f_{-b}^{e \mu \nu} f_{-c}{ }^{f}{ }_{\mu}^{\lambda} \gamma_{5} \gamma_{\nu} T_{A, n, \operatorname{def} \lambda}$ & 59 & 285 & $i \epsilon^{a b c} \bar{B}_{a}{ }^{d} u_{b}{ }^{e \mu} u^{f g \nu} \chi_{-d f} D_{\mu} T_{A, n, c e g \nu}$ & & 577 \\
\hline$\epsilon^{a b c} \bar{B}_{a}^{d} f_{-b}{ }^{e \mu \nu} f_{-d}{ }^{f}{ }^{\lambda} \gamma_{5} \gamma_{\nu} T_{A, n, c e f \lambda}$ & 60 & 286 & $i \epsilon^{a b c} \bar{B}_{a}{ }^{d} u_{b}{ }^{e \mu} u^{f g \nu} \chi_{-d f} D_{\nu} T_{A, n, \text { ceg } \mu}$ & & 578 \\
\hline$\epsilon^{a b c} \bar{B}_{a}^{d} f_{-b}^{e \mu \nu} f_{-e^{f}{ }_{\mu}^{\lambda} \gamma_{5} \gamma_{\nu} T_{A, n, c d f \lambda}}$ & & 287 & $i \epsilon^{a b c} \bar{B}_{a}{ }^{d} u_{b}{ }^{e \mu} u^{f g \nu} \chi_{-e f} D_{\mu} T_{A, n, c d g \nu}$ & & 579 \\
\hline$\epsilon^{a b c} \bar{B}_{a}^{d} f_{-b}^{e \mu \nu} f_{-e}{ }^{f} \lambda \gamma_{5} \gamma_{\lambda} T_{A, n, c d f \nu}$ & & 288 & $i \epsilon^{a b c} \bar{B}_{a}{ }^{d} u_{b}{ }^{e \mu} u^{f g \nu} \chi_{-e f} D_{\nu} T_{A, n, c d g \mu}$ & & 580 \\
\hline$\epsilon^{a b c} \bar{B}_{a}^{d} f_{-b}^{e \mu \nu} f_{-c}^{f \lambda \rho} \gamma_{5} \gamma_{\mu} D_{\nu \lambda} T_{A, n, \operatorname{def} \rho}$ & 61 & 289 & $i \epsilon^{a b c} \bar{B}_{a}{ }^{d} u_{b}{ }^{e \mu} u^{f g \nu} \chi_{-g f} D_{\mu} T_{A, n, c d e \nu}$ & & 581 \\
\hline$\epsilon^{a b c} \bar{B}_{a}^{d} f_{-b}^{e \mu \nu} f_{-d}^{f \lambda \rho} \gamma_{5} \gamma_{\mu} D_{\nu \lambda} T_{A, n, c e f \rho}$ & 62 & 290 & $i \epsilon^{a b c} \bar{B}_{a}{ }^{d} u_{b}{ }^{e \mu} u^{f g \nu} \chi_{-g f} D_{\nu} T_{A, n, c d e \mu}$ & & 582 \\
\hline$\epsilon^{a b c} \bar{B}_{a}^{d} f_{-b}{ }^{e \mu \nu} f_{-e}{ }^{f \lambda \rho} \gamma_{5} \gamma_{\mu} D_{\nu \lambda} T_{A, n, c d f \rho}$ & & 291 & $i \epsilon^{a b c} \bar{B}_{a}^{d} u_{b}{ }^{e \mu} u_{c}{ }^{f \nu} \chi_{-, s} D_{\mu} T_{A, n, d e f \nu}$ & 201 & 583 \\
\hline$\epsilon^{a b c} \bar{B}_{a}^{d} f_{-b}{ }^{e \mu \nu} f_{-e}{ }^{f \lambda \rho} \gamma_{5} \gamma_{\lambda} D_{\mu \rho} T_{A, n, c d f \nu}$ & & 292 & $i \epsilon^{a b c} \bar{B}_{a}^{d} u_{b}{ }^{e \mu} u_{d}{ }^{f \nu} \chi_{-, s} D_{\mu} T_{A, n, c e f \nu}$ & 202 & 584 \\
\hline$\epsilon^{a b c} \bar{B}_{a}^{d} h_{b}{ }^{e \mu \nu} f_{-c}{ }^{f}{ }_{\mu}^{\lambda} \gamma_{5} \gamma_{\nu} T_{A, n, \operatorname{def} \lambda}$ & 63 & 293 & $i \epsilon^{a b c} \bar{B}_{a}^{d} u_{b}^{e \mu} u_{e}^{f \nu} \chi_{-, s} D_{\mu} T_{A, n, c d f \nu}$ & & 585 \\
\hline$\epsilon^{a b c} \bar{B}_{a}{ }^{d} h_{b}{ }^{e \mu \nu} f_{-c}{ }^{f}{ }_{\mu}^{\lambda} \gamma_{5} \gamma_{\lambda} T_{A, n, d e f \nu}$ & 64 & 294 & $i \epsilon^{a b c} \bar{B}_{a}{ }^{d} u_{b}{ }^{e \mu} u_{e}{ }^{f \nu} \chi_{-, s} D_{\nu} T_{A, n, c d f \mu}$ & & 586 \\
\hline$\epsilon^{a b c} \bar{B}_{a}^{d} h_{b}{ }^{e \mu \nu} f_{-d^{f}}{ }^{\lambda}{ }^{\lambda} \gamma_{5} \gamma_{\nu} T_{A, n, c e f \lambda}$ & 65 & 295 & $i \epsilon^{a b c} \bar{B}_{a}^{d} f_{-b}^{e \mu \nu} \chi_{-c}{ }^{f} \gamma_{5} \gamma_{\mu} T_{A, n, d e f \nu}$ & 203 & 587 \\
\hline$\epsilon^{a b c} \bar{B}_{a}^{d} h_{b}{ }^{e \mu \nu} f_{-d}{ }^{f}{ }_{\mu}^{\lambda} \gamma_{5} \gamma_{\lambda} T_{A, n, c e f \nu}$ & 66 & 296 & $i \epsilon^{a b c} \bar{B}_{a}^{d} f_{-b}{ }^{e \mu \nu} \chi_{-d}{ }^{f} \gamma_{5} \gamma_{\mu} T_{A, n, c e f \nu}$ & 204 & 588 \\
\hline$\epsilon^{a b c} \bar{B}_{a}{ }^{d} h_{b}{ }^{e \mu \nu} f_{-e^{f}{ }_{\mu}^{\lambda} \gamma_{5} \gamma_{\nu} T_{A, n, c d f \lambda}}$ & & 297 & $i \epsilon^{a b c} \bar{B}_{a}^{d} f_{-b}^{e \mu \nu} \chi_{-e}{ }^{f} \gamma_{5} \gamma_{\mu} T_{A, n, c d f \nu}$ & & 589 \\
\hline$\epsilon^{a b c} \bar{B}_{a}{ }^{d} h_{b}{ }^{e \mu \nu} f_{-e^{f}{ }_{\mu}^{\lambda} \gamma_{5} \gamma_{\lambda} T_{A, n, c d f \nu}}$ & & 298 & $i \epsilon^{a b c} \bar{B}_{a}{ }^{d} f_{-}{ }^{e f \mu \nu} \chi_{-b e} \gamma_{5} \gamma_{\mu} T_{A, n, c d f \nu}$ & & 590 \\
\hline$\epsilon^{a b c} \bar{B}_{a}{ }^{d} h^{e f \mu \nu} f_{-b e \mu}{ }^{\lambda} \gamma_{5} \gamma_{\nu} T_{A, n, c d f \lambda}$ & & 299 & $i \epsilon^{a b c} \bar{B}_{a}{ }^{d} h_{b}{ }^{e \mu \nu} \chi_{-c}{ }^{f} \gamma_{5} \gamma_{\mu} T_{A, n, \text { def }}$ & 205 & 591 \\
\hline$\epsilon^{a b c} \bar{B}_{a}{ }^{d} h^{e f \mu \nu} f_{-b e \mu}{ }^{\lambda} \gamma_{5} \gamma_{\lambda} T_{A, n, c d f \nu}$ & & 300 & $i \epsilon^{a b c} \bar{B}_{a}{ }^{d} h_{b}{ }^{e \mu \nu} \chi_{-d}{ }^{f} \gamma_{5} \gamma_{\mu} T_{A, n, c e f \nu}$ & 206 & 592 \\
\hline$\epsilon^{a b c} \bar{B}_{a}{ }^{d} h_{b}{ }^{e \mu \nu} f_{-c}{ }^{f \lambda \rho} \gamma_{5} \gamma_{\mu} D_{\nu \lambda} T_{A, n, \operatorname{def} \rho}$ & 67 & 301 & $i \epsilon^{a b c} \bar{B}_{a}{ }^{d} h_{b}{ }^{e \mu \nu} \chi_{-e}{ }^{f} \gamma_{5} \gamma_{\mu} T_{A, n, c d f \nu}$ & & 593 \\
\hline$\epsilon^{a b c} \bar{B}_{a}{ }^{d} h_{b}{ }^{e \mu \nu} f_{-c}{ }^{f \lambda \rho} \gamma_{5} \gamma_{\lambda} D_{\mu \nu} T_{A, n, d e f \rho}$ & 68 & 302 & $i \epsilon^{a b c} \bar{B}_{a}{ }^{d} h^{e f \mu \nu} \chi_{-b e} \gamma_{5} \gamma_{\mu} T_{A, n, c d f \nu}$ & & 594 \\
\hline$\epsilon^{a b c} \bar{B}_{a}{ }^{d} h_{b}{ }^{e \mu \nu} f_{-c} f \lambda \rho \gamma_{5} \gamma_{\lambda} D_{\mu \rho} T_{A, n, d e f \nu}$ & 69 & 303 & $i \epsilon^{a b c} \bar{B}_{a}^{d} f_{-b}{ }^{e \mu \nu} \chi_{-, s} \gamma_{5} \gamma_{\mu} T_{A, n, c d e \nu}$ & 207 & 595 \\
\hline$\epsilon^{a b c} \bar{B}_{a}{ }^{d} h_{b}{ }^{e \mu \nu} f_{-d}{ }^{f \lambda \rho} \gamma_{5} \gamma_{\mu} D_{\nu \lambda} T_{A, n, c e f \rho}$ & 70 & 304 & $i \epsilon^{a b c} \bar{B}_{a}^{d} h_{b}{ }^{e \mu \nu} \chi_{-, s} \gamma_{5} \gamma_{\mu} T_{A, n, c d e \nu}$ & 208 & 596 \\
\hline$\epsilon^{a b c} \bar{B}_{a}{ }^{d} h_{b}{ }^{e \mu \nu} f_{-d}{ }^{f \lambda \rho} \gamma_{5} \gamma_{\lambda} D_{\mu \nu} T_{A, n, c e f \rho}$ & 71 & 305 & $i \epsilon^{a b c} \bar{B}_{a}{ }^{d} u_{b}{ }^{e \mu} \nabla^{\nu} \chi_{-c}{ }^{f} \gamma_{5} \gamma_{\mu} T_{A, n, \text { def } \nu}$ & 209 & 597 \\
\hline
\end{tabular}


TABLE VII. (Continued)

\begin{tabular}{|c|c|c|c|c|c|}
\hline$P_{n}^{\left(N_{f}, 4\right)}$ & $S U(2)$ & $S U(3)$ & $P_{n}^{\left(N_{f}, 4\right)}$ & $S U(2)$ & $S U(3)$ \\
\hline$\epsilon^{a b c} \bar{B}_{a}{ }^{d} h_{b}{ }^{e \mu \nu} f_{-d}{ }^{f \lambda \rho} \gamma_{5} \gamma_{\lambda} D_{\mu \rho} T_{A, n, c e f \nu}$ & 72 & 306 & $i \epsilon^{a b c} \bar{B}_{a}{ }^{d} u_{b}{ }^{e \mu} \nabla^{\nu} \chi_{-c}{ }^{f} \gamma_{5} \gamma_{\nu} T_{A, n, \text { def } \mu}$ & 210 & 598 \\
\hline$\epsilon^{a b c} \bar{B}_{a}{ }^{d} h_{b}{ }^{e \mu \nu} f_{-e}{ }^{f \lambda \rho} \gamma_{5} \gamma_{\mu} D_{\nu \lambda} T_{A, n, c d f \rho}$ & & 307 & $i \epsilon^{a b c} \bar{B}_{a}^{d} u_{b}{ }^{e \mu} \nabla^{\nu} \chi_{-d}{ }^{f} \gamma_{5} \gamma_{\mu} T_{A, n, c e f \nu}$ & 211 & 599 \\
\hline$\epsilon^{a b c} \bar{B}_{a}{ }^{d} h_{b}{ }^{e \mu \nu} f_{-e}{ }^{f \lambda \rho} \gamma_{5} \gamma_{\lambda} D_{\mu \nu} T_{A, n, c d f \rho}$ & & 308 & $i \epsilon^{a b c} \bar{B}_{a}{ }^{d} u_{b}{ }^{e \mu} \nabla^{\nu} \chi_{-d}{ }^{f} \gamma_{5} \gamma_{\nu} T_{A, n, c e f \mu}$ & 212 & 600 \\
\hline$\epsilon^{a b c} \bar{B}_{a}{ }^{d} h_{b}{ }^{e \mu \nu} f_{-e}{ }^{f \lambda \rho} \gamma_{5} \gamma_{\lambda} D_{\mu \rho} T_{A, n, c d f \nu}$ & & 309 & $i \epsilon^{a b c} \bar{B}_{a}{ }^{d} u_{b}{ }^{e \mu} \nabla^{\nu} \chi_{-e}{ }^{f} \gamma_{5} \gamma_{\mu} T_{A, n, c d f \nu}$ & & 601 \\
\hline$\epsilon^{a b c} \bar{B}_{a}{ }^{d} h^{e f \mu \nu} f_{-b e}{ }^{\lambda \rho} \gamma_{5} \gamma_{\mu} D_{\nu \lambda} T_{A, n, c d f \rho}$ & & 310 & $i \epsilon^{a b c} \bar{B}_{a}{ }^{d} u_{b}{ }^{e \mu} \nabla^{\nu} \chi_{-e}{ }^{f} \gamma_{5} \gamma_{\nu} T_{A, n, c d f \mu}$ & & 602 \\
\hline$\epsilon^{a b c} \bar{B}_{a}{ }^{d} h^{e f \mu \nu} f_{-b e}{ }^{\lambda \rho} \gamma_{5} \gamma_{\lambda} D_{\mu \nu} T_{A, n, c d f \rho}$ & & 311 & $i \epsilon^{a b c} \bar{B}_{a}{ }^{d} u^{e f \mu} \nabla^{\nu} \chi_{-b e} \gamma_{5} \gamma_{\mu} T_{A, n, c d f \nu}$ & & 603 \\
\hline$\epsilon^{a b c} \bar{B}_{a}{ }^{d} h^{e f \mu \nu} f_{-b e^{\lambda \rho} \gamma_{5} \gamma_{\lambda} D_{\mu \rho} T_{A, n, c d f \nu}}$ & & 312 & $i \epsilon^{a b c} \bar{B}_{a}{ }^{d} u^{e f \mu} \nabla^{\nu} \chi_{-b e} \gamma_{5} \gamma_{\nu} T_{A, n, c d f \mu}$ & & 604 \\
\hline$\epsilon^{a b c} \bar{B}_{a}{ }^{d} h_{b}{ }^{e \mu \nu} h_{c}{ }^{f}{ }_{\mu}^{\lambda} \gamma_{5} \gamma_{\nu} T_{A, n, \text { def } \lambda}$ & 73 & 313 & $i \epsilon^{a b c} \bar{B}_{a}{ }^{d} u_{b}{ }^{e \mu} \nabla^{\nu} \chi_{-, s} \gamma_{5} \gamma_{\mu} T_{A, n, c d e \nu}$ & 213 & 605 \\
\hline$\epsilon^{a b c} \bar{B}_{a}{ }^{d} h_{b}{ }^{e \mu \nu} h_{d}{ }^{f}{ }_{\mu}^{\lambda} \gamma_{5} \gamma_{\nu} T_{A, n, c e f \lambda}$ & 74 & 314 & $i \epsilon^{a b c} \bar{B}_{a}^{d} u_{b}^{e \mu} \nabla^{\nu} \chi_{-, s} \gamma_{5} \gamma_{\nu} T_{A, n, c d e \mu}$ & 214 & 606 \\
\hline$\epsilon^{a b c} \bar{B}_{a}{ }^{d} h_{b}{ }^{e \mu \nu} h_{e}{ }^{f}{ }_{\mu}^{\lambda} \gamma_{5} \gamma_{\nu} T_{A, n, c d f \lambda}$ & & 315 & $\epsilon^{a b c} \bar{B}_{a}{ }^{d} f_{+b}{ }^{e \mu \nu} \chi_{-c}{ }^{f} D_{\mu} T_{A, n, \text { def }}$ & 215 & 607 \\
\hline$\epsilon^{a b c} \bar{B}_{a}{ }^{d} h_{b}{ }^{e \mu \nu} h_{e}{ }^{f}{ }_{\mu}^{\lambda} \gamma_{5} \gamma_{\lambda} T_{A, n, c d f \nu}$ & & 316 & $\epsilon^{a b c} \bar{B}_{a}^{d} f_{+b}{ }^{e \mu \nu} \chi_{-d}{ }^{f} D_{\mu} T_{A, n, c e f \nu}$ & 216 & 608 \\
\hline$\epsilon^{a b c} \bar{B}_{a}{ }^{d} h_{b}{ }^{e \mu \nu} h_{c}^{f \lambda} \gamma_{5} \gamma_{\mu} D_{\nu \lambda} T_{A, n, \text { def } \rho}$ & 75 & 317 & $\epsilon^{a b c} \bar{B}_{a}{ }^{d} f_{s,+}{ }^{\mu \nu} \chi_{-b}{ }^{e} D_{\mu} T_{A, n, c d e \nu}$ & 217 & \\
\hline$\epsilon^{a b c} \bar{B}_{a}{ }^{d} h_{b}{ }^{e \mu \nu} h_{d}{ }^{f \lambda \rho} \gamma_{5} \gamma_{\mu} D_{\nu \lambda} T_{A, n, \text { cef } \rho}$ & 76 & 318 & $\epsilon^{a b c} \bar{B}_{a}{ }^{d} f_{+b}{ }^{e \mu \nu} \chi_{-e}{ }^{f} D_{\mu} T_{A, n, c d f \nu}$ & & 609 \\
\hline$\epsilon^{a b c} \bar{B}_{a}{ }^{d} h_{b}{ }^{e \mu \nu} h_{e}{ }^{f \lambda \rho} \gamma_{5} \gamma_{\mu} D_{\nu \lambda} T_{A, n, c d f \rho}$ & & 319 & $\epsilon^{a b c} \bar{B}_{a}{ }^{d} f_{+}{ }^{e f \mu \nu} \chi_{-b e} D_{\mu} T_{A, n, c d f \nu}$ & & 610 \\
\hline$\epsilon^{a b c} \bar{B}_{a}{ }^{d} h_{b}{ }^{e \mu \nu} h_{e}{ }^{f \lambda \rho} \gamma_{5} \gamma_{\lambda} D_{\mu \nu} T_{A, n, c d f \rho}$ & & 320 & $\epsilon^{a b c} \bar{B}_{a}{ }^{d} f_{+b}{ }^{e \mu \nu} \chi_{-, s} D_{\mu} T_{A, n, c d e \nu}$ & 218 & 611 \\
\hline$\epsilon^{a b c} \bar{B}_{a}{ }^{d} u_{b}{ }^{e \mu} \nabla_{\mu} f_{-c}{ }^{f \nu \lambda} \gamma_{5} \gamma_{\nu} T_{A, n, \text { def }}$ & 77 & 321 & & & \\
\hline
\end{tabular}

[1] S. Weinberg, Phenomenological Lagrangians, Physica A (Amsterdam) 96, 327 (1979).

[2] J. Gasser and H. Leutwyler, Chiral perturbation theory to one loop, Ann. Phys. (N.Y.) 158, 142 (1984).

[3] J. Gasser and H. Leutwyler, Chiral perturbation theory: Expansions in the mass of the strange quark, Nucl. Phys. B250, 465 (1985).

[4] J. Gasser, M. E. Sainio, and A. Švarc, Nucleons with chiral loops, Nucl. Phys. B307, 779 (1988).

[5] H.W. Fearing and S. Scherer, Extension of the chiral perturbation theory meson Lagrangian to order $p^{6}$, Phys. Rev. D 53, 315 (1996).

[6] J. Bijnens, G. Colangelo, and G. Ecker, The mesonic chiral Lagrangian of order $p^{6}$, J. High Energy Phys. 02 (1999) 020.

[7] C. Haefeli, M. A. Ivanov, M. Schmid, and G. Ecker, On the mesonic Lagrangian of order $p^{6}$ in chiral $\mathrm{SU}(2)$, arXiv:0705.0576.

[8] T. Ebertshäuser, H. W. Fearing, and S. Scherer, The anomalous chiral perturbation theory meson Lagrangian to order $p^{6}$ reexamined, Phys. Rev. D 65, 054033 (2002).

[9] J. Bijnens, L. Girlanda, and P. Talavera, The anomalous chiral Lagrangian of order $p^{6}$, Eur. Phys. J. C 23, 539 (2002).

[10] O. Catà and V. Mateu, Chiral perturbation theory with tensor sources, J. High Energy Phys. 09 (2007) 078.

[11] P. Herrera-Siklódy, J. Latorre, P. Pascual, and J. Taron, Chiral effective Lagrangian in the large $N_{c}$ limit: the nonet case, Nucl. Phys. B497, 345 (1997).

[12] S.-Z. Jiang, F.-J. Ge, and Q. Wang, Full pseudoscalar mesonic chiral Lagrangian at $p^{6}$ order under the unitary group, Phys. Rev. D 89, 074048 (2014).
[13] A. Krause, Baryon matrix elements of the vector current in chiral perturbation theory, Helv. Phys. Acta 63, 3 (1990).

[14] G. Ecker, Chiral invariant renormalization of the pionnucleon interaction, Phys. Lett. B 336, 508 (1994).

[15] N. Fettes, U.-G. Meißner, and S. Steininger, Pion-nucleon scattering in chiral perturbation theory (I): Isospin-symmetric case, Nucl. Phys. A640, 199 (1998).

[16] U.-G. Meißner, G. Muller, and S. Steininger, Renormalization of the chiral pion-nucleon Lagrangian beyond next-toleading order, Ann. Phys. (N.Y.) 279, 1 (2000).

[17] N. Fettes, U.-G. Meißner, M. Mojžišs, and S. Steininger, The chiral effective pion nucleon Lagrangian of order $p^{4}$, Ann. Phys. (N.Y.) 283, 273 (2000).

[18] J. A. Oller, M. Verbeni, and J. Prades, Meson-baryon effective chiral Lagrangians to $\mathcal{O}\left(q^{3}\right)$, J. High Energy Phys. 09 (2006) 079.

[19] M. Frink and U.-G. Meißner, On the chiral effective mesonbaryon Lagrangian at third order, Eur. Phys. J. A 29, 255 (2006).

[20] S.-Z. Jiang, Q.-S. Chen, and Y.-R. Liu, Meson-baryon effective chiral Lagrangians at order $p^{4}$, Phys. Rev. D 95, 014012 (2017).

[21] T. R. Hemmert, B. R. Holstein, and J. Kambor, Heavy baryon chiral perturbation theory with light deltas, J. Phys. G 24, 1831 (1998).

[22] S.-Z. Jiang, Y.-R. Liu, and H.-Q. Wang, Chiral Lagrangian with $\Delta(1232)$ to one loop, Phys. Rev. D 97, 014002 (2018).

[23] E. Jenkins, Baryon masses in chiral perturbation theory, Nucl. Phys. B368, 190 (1992).

[24] R. F. Lebed, Baryon decuplet mass relations in chiral perturbation theory, Nucl. Phys. B430, 295 (1994). 
[25] X.-L. Ren, L. Geng, J. Meng, and H. Toki, Virtual decuplet effects on octet baryon masses in covariant baryon chiral perturbation theory, Phys. Rev. D 87, 074001 (2013).

[26] X.-L. Ren, L.-S. Geng, and J. Meng, Decuplet baryon masses in covariant baryon chiral perturbation theory, Phys. Rev. D 89, 054034 (2014).

[27] E. E. Jenkins, M. Luke, A. V. Manohar, and M. J. Savage, Chiral perturbation theory analysis of the baryon magnetic moments, Phys. Lett. B 302, 482 (1993).

[28] L. S. Geng, J. M. Camalich, and M. J. V. Vacas, Leadingorder decuplet contributions to the baryon magnetic moments in chiral perturbation theory, Phys. Lett. B 676, 63 (2009).

[29] L. S. Geng, J. M. Camalich, and M. J. V. Vacas, Electromagnetic structure of the lowest-lying decuplet resonances in covariant chiral perturbation theory, Phys. Rev. D 80, 034027 (2009).

[30] H.-S. Li, Z.-W. Liu, X.-L. Chen, W.-Z. Deng, and S.-L. Zhu, Magnetic moments and electromagnetic form factors of the decuplet baryons in chiral perturbation theory, Phys. Rev. D 95, 076001 (2017).

[31] Y.-R. Liu and S.-L. Zhu, Decuplet contribution to the meson-baryon scattering lengths, Eur. Phys. J. C 52, 177 (2007).

[32] Z.-W. Liu, Y.-R. Liu, and S.-L. Zhu, Pseudoscalar meson and decuplet baryon scattering lengths, Phys. Rev. D 83, 034004 (2011).

[33] M. N. Butler, M. J. Savage, and R. P. Springer, E2/M1 mixing ratio of $\Delta \rightarrow N \gamma$ and hyperon resonance radiative decay, Phys. Lett. B 304, 353 (1993).

[34] H.-S. Li, Z.-W. Liu, X.-L. Chen, W.-Z. Deng, and S.-L. Zhu, Decuplet to octet baryon transitions in chiral perturbation theory, arXiv:1706.06458.

[35] D. Arndt and B. C. Tiburzi, Electromagnetic properties of the baryon decuplet in quenched and partially quenched chiral perturbation theory, Phys. Rev. D 68, 114503 (2003).

[36] D. Arndt and B. C. Tiburzi, Baryon decuplet to octet electromagnetic transitions in quenched and partially quenched chiral perturbation theory, Phys. Rev. D 69, 014501 (2004).

[37] B. C. Tiburzi and A. Walker-Loud, Decuplet baryon masses in partially quenched chiral perturbation theory, Nucl. Phys. A748, 513 (2005).

[38] J. Haidenbauer, S. Petschauer, N. Kaiser, U.-G. Meißner, and W. Weise, Scattering of decuplet baryons in chiral effective field theory, Eur. Phys. J. C 77, 760 (2017).

[39] E. Jenkins and A. V. Manohar, Chiral corrections to the baryon axial currents, Phys. Lett. B 259, 353 (1991).

[40] H.-B. Tang and P. J. Ellis, Redundance of delta isobar parameters in effective field theories, Phys. Lett. B 387, 9 (1996).

[41] W. Rarita and J. Schwinger, On a theory of particles with half integral spin, Phys. Rev. 60, 61 (1941).
[42] P. A. Moldauer and K. M. Case, Properties of half-integral spin Dirac-Fierz-Pauli particles, Phys. Rev. 102, 279 (1956).

[43] C. Fronsdal, On the theory of higher spin fields, Il Nuovo Cimento 9, 416 (1958).

[44] A. Aurilia and H. Umezawa, Theory of high-spin fields, Phys. Rev. 182, 1682 (1969).

[45] P. Van Nieuwenhuizen, Supergravity, Phys. Rep. 68, 189 (1981).

[46] H. T. Williams, Misconceptions regarding spin 3/2, Phys. Rev. C 31, 2297 (1985).

[47] M. Benmerrouche, R.M. Davidson, and N.C. Mukhopadhyay, Problems of describing spin $3 / 2$ baryon resonances in the effective Lagrangian theory, Phys. Rev. C 39, 2339 (1989).

[48] V. Pascalutsa, On the interaction of spin $3 / 2$ particles, arXiv:hep-ph/9412321.

[49] H. Haberzettl, Propagation of a massive spin-3/2 particle, arXiv:nucl-th/9812043.

[50] T. Pilling, Symmetry of massive Rarita-Schwinger fields, Int. J. Mod. Phys. A 20, 2715 (2005).

[51] A. E. Kaloshin and V. P. Lomov, Rarita-Schwinger field: Dressing procedure and spin-parity of components, Phys. At. Nucl. 69, 541 (2006).

[52] T. R. Hemmert, B. R. Holstein, and J. Kambor, Systematic $1 / M$ expansion for spin $3 / 2$ particles in baryon chiral perturbation theory, Phys. Lett. B 395, 89 (1997).

[53] T. R. Hemmert, Ph.D. thesis, Massachusetts University, Amherst, 1997.

[54] C. Hacker, N. Wies, J. Gegelia, and S. Scherer, Including the $\Delta(1232)$ resonance in baryon chiral perturbation theory, Phys. Rev. C 72, 055203 (2005).

[55] H. Krebs, E. Epelbaum, and U. G. Meißner, Redundancy of the off-shell parameters in chiral effective field theory with explicit spin-3/2 degrees of freedom, Phys. Lett. B 683, 222 (2010).

[56] S. Scherer and M.R. Schindler, A Primer for Chiral Perturbation Theory (Springer-Verlag, Berlin, 2012), Vol. 830.

[57] S. Kamefuchi, L. O'Raifeartaigh, and A. Salam, Change of variables and equivalence theorems in quantum field theories, Nucl. Phys. 28, 529 (1961).

[58] L. M. Nath, B. Etemadi, and J. D. Kimel, Uniqueness of the interaction involving spin $3 / 2$ particles, Phys. Rev. D 3, 2153 (1971).

[59] V. I. Borodulin, R. N. Rogalyov, and S. R. Slabospitskii, CORE 3.1 (COmpendium of RElations, Version 3.1), arXiv: 1702.08246.

[60] H.-S. Zong, F. Wang, and J.-L. Ping, The generalized Fierz transformation and its application, Commun. Theor. Phys. 22, 479 (1994).

[61] M. Holmberg and S. Leupold, The relativistic chiral Lagrangian for decuplet and octet baryons at next-to-leading order, arXiv:1802.05168. 